Linköping Studies in Science and Technology

Dissertation No. 1646, 2015

\title{
Variational Tensor-Based Models for Image Diffusion in Non-Linear Domains
}

\author{
Freddie Åström
}

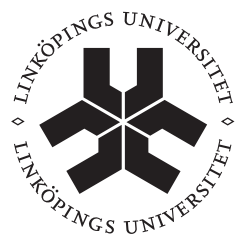

\section{Linköping University \\ INSTITUTE OF TECHNOLOGY}

Department of Electrical Engineering

Linköping University, SE-581 83 Linköping, Sweden

Linköping March 2015 
Variational Tensor-Based Models for Image Diffusion in Non-Linear Domains

(C) 2015 Freddie Åström

\author{
Computer Vision Laboratory \\ Department of Electrical Engineering \\ Linköping University \\ SE-581 83 Linköping \\ Sweden
}

ISBN 978-91-7519-113-3

ISSN 0345-7524 


\section{Abstract}

This dissertation addresses the problem of adaptive image filtering. Although the topic has a long history in the image processing community, researchers continuously present novel methods to obtain ever better image restoration results.

With an expanding market for individuals who wish to share their everyday life on social media, imaging techniques such as compact cameras and smart phones are important factors. Naturally, every producer of imaging equipment desires to exploit cheap camera components while supplying high quality images. One step in this pipeline is to use sophisticated imaging software including, e.g., noise reduction to reduce manufacturing costs, while maintaining image quality.

This thesis is based on traditional formulations such as isotropic and tensorbased anisotropic diffusion for image denoising. The difference from main-stream denoising methods is that this thesis explores the effects of introducing contextual information as prior knowledge for image denoising into the filtering schemes. To achieve this, the adaptive filtering theory is formulated from an energy minimization standpoint. The core contributions of this work is the introduction of a novel tensor-based functional which unifies and generalises standard diffusion methods. Additionally, the explicit Euler-Lagrange equation is derived which, if solved, yield the stationary point for the minimization problem. Several aspects of the functional are presented in detail which include, but are not limited to, tensor symmetry constraints and convexity. Also, the classical problem of finding a variational formulation to a given tensor-based partial differential equation is studied.

The presented framework is applied in problem formulation that includes nonlinear domain transformation, e.g., visualization of medical images. Additionally, the framework is also used to exploit locally estimated probability density functions or the channel representation to drive the filtering process.

Furthermore, one of the first truly tensor-based formulations of total variation is presented. The key to the formulation is the gradient energy tensor, which does not require spatial regularization of its tensor components. It is shown empirically in several computer vision applications, such as corner detection and optical flow, that the gradient energy tensor is a viable replacement for the commonly used structure tensor. Moreover, the gradient energy tensor is used in the traditional tensor-based anisotropic diffusion scheme. This approach results in significant improvements in computational speed when the scheme is implemented on a graphical processing unit compared to using the commonly used structure tensor. 


\section{Populärvetenskaplig sammanfattning}

I dagens samhälle har bildens betydelse blivit en viktig faktor i bland annat sociala medier. Ett allt större utbud av billiga digitalkameror och mobiltelefoner är en viktig faktor för denna utveckling. En anledning är att bildåtergivande tekniker är kostnadseffektiva, men ändå producerar högkvalitativa bilder. Till viss del beror det på att tillverkare använder sig av sofistikerade bildbehandlingsmetoder för att minska komponentkostnader. En viktig del i maskineriet för att producera en bild är brusreduceringsmetoder.

Den här avhandlingen fokuserar på metoder för adaptiv brusreducering. Trots att ämnet historiskt sett är välstuderat fortsätter forskare att uppfinna metoder och algoritmer som ger än bättre brusreducering än vad man tidigare trott var möjligt.

Denna avhandling tar avstamp i traditionella brusreduceringsmetoder som värmeledningsmodellen och tensor-baserad diffusion. Skillnaden mot befintliga avbrusningsmetoder är att denna avhandling formulerar metoder för att inkludera kontextuell och förutbestämd information som är relevant för avbusningsproblemet. Detta betyder att arbetet utforskar möjligheten att inkludera begränsningar i metodformuleringen som kan modelleras från den tänkta tillämpningen. Huvudbidraget i denna avhandling är en ny tensorbaserad energifunktional som generaliserar kända diffusionsmetoder. För denna funktional härleder vi Euler-Lagrange ekvationen som ger ett nödvändigt villkor för att hitta den lösning som ger minst energi. Flera resultat kan härledas från funktionalformuleringen, t.ex. villkor på tensor-symmetri och konvexitet. Även det klassiska problemet att hitta en variationsformulering tillhörande en tensorbaserad partiell differentialekvation har studerats.

Tillämpningar som speciellt behandlas i det presenterade ramverket inkluderar visualisering av medicinska bilder och avbrusning med hjälp av sannolikhetsfördelningar skattade från lokalt homogena områden.

Ett ytterligare signifikant metodbidrag som presenteras i denna avhandling är en tensorbaserad variationsformulering för total variation. Det som möjliggör denna formulering är betraktandet av en mindre känd tensor, gradient energi tensorn. Empiriska resultat visar att denna tensor även kan tillämpas i klassiska datorseendetillämpningar så som hörnpunktsdetektering och optiskt flöde. Detta visar att tensorn kan användas som ett alternativ till strukturtensorn även i andra relevanta tillämpningar. Vidare används gradient energi tensorn i den klassiska formuleringen av tensorbaserad diffusion vilket möjliggör realtidsdiffusion där diffusionsekvationen löses med hjälpa av en grafikprocessor. 


\section{Acknowledgements}

Without the continued support of my supervisor Prof. Michael Felsberg and cosupervisor associate Prof. George Baravdish this thesis would not have become the work that it is today. I am truly grateful for their encouragements and willingness to share their scientific knowledge. It would not have been the same without you, thank you!

During my studies I have meet a lot of interesting people, some has influenced my work a great deal. I want to mention assistant Prof. Vasileios Zografos not only for all late night discussions, great advice and suggestions on research related topics, but also for being a friend.

I am very happy to have had guidance from Fahad Khan, research fellow, associate Prof. Per-Erik Forssén, associate Prof. Reiner Lenz, and Prof. Rudolf Mester all of whom have been supportive and provided good references. I want to thank all past and present members of the Computer Vision Laboratory for providing a comfortable and friendly atmosphere in which good research can thrive. Additionally, I am grateful to the lab's current $\mathrm{PhD}$ students for reviewing parts of this thesis.

I am thankful to have visited Jülich Forschungszentrum, Germany under the supervision of Hanno Scharr, PhD. During my visit I worked closely with Christian Heinemann, at the time PhD student of Hanno, who introduced me to the fascinating topic of channel representation.

Furthermore, I want to thank Prof. Anders Ynnerman, for by a seemingly arbitrary path of unlikely events having suggested an early motivation for the idea of "a mapping function", which at the time was denoted a "transfer function". However, the actual realization, from early attempts to the formalism that is presented in this thesis, is none of his fault.

Lastly, I take this opportunity to thank my family for your love and support during this, at times, challenging period of my life.

This research has received funding from the Swedish Research Council through grants for the projects Non-linear adaptive color image processing (NACIP), Visualization adaptive Iterative Denoising of Images (VIDI), Energy Models for Computational Cameras $\left(\mathrm{EMC}^{2}\right)$ and from the ECs 7th Framework Programme (FP7/2007-2013), grant agreement 247947 (GARNICS). Also, this work has been conducted within (in collaboration with) the Center for Medical Image Science and Visualization (CMIV) at Linköping University, Sweden. CMIV is acknowledged for provision of financial support and access to leading edge research infrastructure. 


\section{Abbreviations}

BM3D Block-matching via sparse 3D representation

CBM3D Colour block-matching via sparse 3D representation

CDF Cumulative distribution function

CS Channel smoothing

CUDA Compute unified device architecture

D3 Density driven diffusion

E-L Euler Lagrange

EAD Extended anisotropic diffusion

GET Gradient energy tensor

GETm Gradient energy tensor with mapping function

GETV Gradient energy total variation

GETVm Gradient energy total variation with mapping function

GPU Graphics processing unit

LCD Linear channel diffusion

LD Linear diffusion

MF Median filter

MS Mean shift

MSE Mean squared error

NC Necessary condition

NLCD Non-linear channel diffusion

NLM Non-local means

PDE Partial differential equation

PDF Probability density function

PM Perona and Malik

PSNR Peak signal-to-noise ratio

SC Sufficient condition

SSIM Structural similarity

TIF Targeted iterative filtering

TR Trace-based diffusion

TV Total variation

TVm Total variation with mapping function 


\section{Contents}

List of Statements xiv

1 Introduction 1

1.1 Motivation . . . . . . . . . . . . . . . . . 1

1.2 Organisation of Thesis $\ldots \ldots \ldots \ldots \ldots \ldots \ldots$

1.3 Included publications $\ldots \ldots \ldots \ldots \ldots \ldots$

1.4 Related publications . . . . . . . . . . . . . . . . . . . 11

Part I: Preliminaries

$\begin{array}{lll}2 & \text { Background } & 13\end{array}$

2.1 Denoising methods in image processing. . . . . . . . . . . . . . . 13

2.2 Preliminaries $\ldots \ldots \ldots \ldots \ldots \ldots$

2.3 Additional remarks . . . . . . . . . . . . . . . . . . . . . . . . . . 24

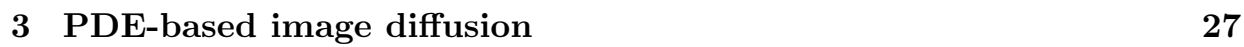

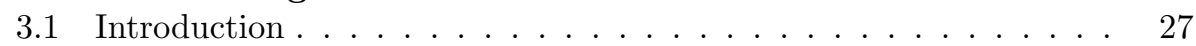

3.2 Isotropic diffusion $\ldots \ldots \ldots \ldots \ldots \ldots$

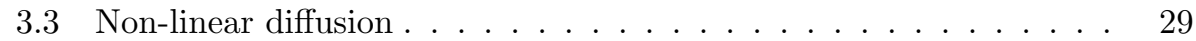

3.4 Tensor-based diffusion $\ldots \ldots \ldots \ldots$

Part II: Image diffusion in non-linear domains

4 Tensor-valued diffusion in non-linear domains 33

4.1 Domain-dependent non-linear transformations . . . . . . . . . . . 33

4.2 Noise estimation in the transformed domain . . . . . . . . . . . . 35

4.3 Tensor-valued domain-dependent functional . . . . . . . . . . . 37

4.4 Study of the inverse problem $\ldots \ldots \ldots \ldots \ldots$

5 Scalar-valued diffusion in non-linear domains 49

$5.1 \quad p$-Norm formulation $\ldots \ldots \ldots \ldots \ldots \ldots \ldots$

5.2 Isotropic diffusion in non-linear domains . . . . . . . . . . . . 50

5.3 Non-linear total variation $\ldots \ldots \ldots \ldots \ldots \ldots$ 
$\begin{array}{lll}6 & \text { Prior information in non-linear image filtering } & 57\end{array}$

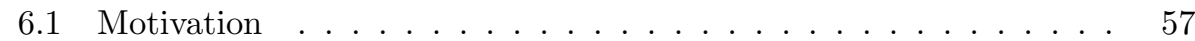

6.2 Oversegmentation . . . . . . . . . . . . . . . . . 58

6.3 Density driven diffusion . . . . . . . . . . . . . . . . . . . 60

$\begin{array}{lll}7 & \text { Channel-based image processing } & 63\end{array}$

7.1 Motivation . . . . . . . . . . . . . . . . . . 63

7.2 Channel smoothing . . . . . . . . . . . . . . . . 64

7.3 Isotropic channel-based regularization . . . . . . . . . . . . . . . . 65

7.4 Non-linear channel-based regularization . . . . . . . . . . . . . . 68

Part III: Applications of the gradient energy tensor

8 Gradient energy tensor $\quad 69$

8.1 Definition of tensor . . . . . . . . . . . . . . . . . . . . . 69

8.2 Orientation estimation . . . . . . . . . . . . . . . . 71

8.3 Corner detection and optical flow . . . . . . . . . . . . . 73

$\begin{array}{lll}9 & \text { Gradient energy total variation } & \mathbf{7 7}\end{array}$

9.1 Tensor-based extensions of total variation . . . . . . . . . . . . . 78

9.2 Definition of gradient energy total variation . . . . . . . . . . . . 78

9.3 Generalised formulation . . . . . . . . . . . . . . . . 81

Part IV: Colour representation, implementation and evaluation

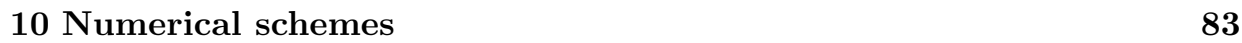

10.1 Numerical implementation . . . . . . . . . . . . . . . . . . . . 83

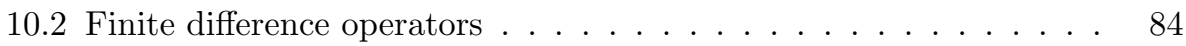

10.3 Approximation of the divergence operator . . . . . . . . . . . . 86

$\begin{array}{ll}11 \text { Evaluation framework } & 91\end{array}$

11.1 Colour space representation . . . . . . . . . . . . . . . . . . . 91

11.2 Image quality metrics . . . . . . . . . . . . . . . . . . . . . . . . . . . . . . . . . . . . . . 93

11.3 Image datasets . . . . . . . . . . . . . . . . . . . . . . . . . . . . . . . . . . . . . . . . . . . . 95

11.4 Evaluation setup . . . . . . . . . . . . . . . . . . 96 96

$\begin{array}{ll}12 \text { Scalar-valued applications } & 99\end{array}$

12.1 Non-linear isotropic enhancement of medical images . . . . . . . . 99

12.2 Channel-based regularization . . . . . . . . . . . . . . 102

12.3 Enhancement via density estimates . . . . . . . . . . . . . 106

$\begin{array}{lr}13 \text { Tensor-valued applications } & 111\end{array}$

13.1 Extended anisotropic diffusion . . . . . . . . . . . . . . . . . . . . 111

13.2 Gradient energy total variation . . . . . . . . . . . . . . . . . . . 115

13.3 Significance analysis of the mapping function . . . . . . . . . . . . 121 
14 Fast image diffusion on the GPU 127

14.1 Introduction to GPU computing . . . . . . . . . . . . . . . . . . . . 127

14.2 Image diffusion on the GPU . . . . . . . . . . . . . . . . . . . . 128

14.3 Implementation details . . . . . . . . . . . . . . . . . . . . . . . . . . . . . . . . . 130

14.4 Results. . . . . . . . . . . . . . . . . . . . 132

Part V: Concluding remarks

15 Conclusions 135

15.1 Summary of the results . . . . . . . . . . . . . . . . . 135

15.2 Future work . . . . . . . . . . . . . . . . . . . . . 137

\begin{tabular}{ll}
\hline A Appendices & 139
\end{tabular}

A.1 Proof Theorem 2 . . . . . . . . . . . . . . . . . . . . 139

A.2 Extended proof Lemma 1 . . . . . . . . . . . . . . . . . . . . . 143

A.3 Derivation GETVm . . . . . . . . . . . . . . . . . . . . . . . 144

$\begin{array}{ll}16 \text { Bibliography } & 149\end{array}$ 


\section{List of Statements}

$1 \quad$ Theorem (Spectral theorem) $\ldots \ldots \ldots \ldots$

2 Theorem (Unifying functional) $\ldots \ldots \ldots \ldots$. . . . . . . . . 37

$1 \quad$ Corollary (Constraints symmetric tensor) . . . . . . . . . . . . . 40

$1 \quad$ Lemma (Contraction of divergence forms) . . . . . . . . . . . . 40

$2 \quad$ Corollary (Contraction with symmetry constraint) . . . . . . . . . 41

$3 \quad$ Corollary (Convex functional) $\quad \ldots \ldots \ldots \ldots \ldots$. . . . . . . . . . 42

1 Definition (Extended anisotropic diffusion) . . . . . . . . . . . . 44

$3 \quad$ Theorem (NC for PDE to functional) $\ldots \ldots \ldots \ldots \ldots$. . . . . . . . 44

$4 \quad$ Corollary (NC conditions for PDE to functional) $\ldots \ldots \ldots$. . . . 46

\begin{tabular}{|l|ll}
\hline 4 Theorem (SC local minimum for isotropic diffusion in non-linear \\
\hline
\end{tabular}

2 Lemma (Condition GET positive semi-definite) $\ldots \ldots \ldots$. . . . 71

$2 \quad$ Definition (Gradient energy total variation) $\ldots \ldots \ldots \ldots 78$

3 Definition (GETV generalised formulation) $\ldots \ldots$. . . . . . . 81 


\section{Chapter 1}

\section{Introduction}

\subsection{Motivation}

One of the first ever recorded photographs was taken in the mid-19th century. The imaging device was a crude construction and produced poor images. The basic principle of the first imaging devices was to collect incident light on light-sensitive substances. The substance responded to the light and thus formed images. Since the substance had a slow reaction time, long exposure intervals were required, in some cases hours, or even days. The long processing time produced low quality images suffering from poor resolution, noise and motion artefacts.

Modern imaging devices do not anymore use active substances in the imaging process. Instead they typically use sensor elements known as charge-coupled devices (CCDs) or complementary metal-oxide semiconductor (CMOS), to measure small variations in electrical current. An image is made up of pixels, and vaguely described, each pixel corresponds to a sensor element. In order to construct a good image, other factors of the camera must be taken into account such as the quality of the optical lens.

This means that, in principle, the better sensor elements and lens the camera is equipped with, the better the scene will be reflected in the final image. However, with improved imaging equipment, the more expensive the camera will be. Therefore, it is important and relevant for camera manufacturers to use sophisticated algorithms to post-process the image data to create visually appealing images. This sort of post-processing includes, e.g., motion compensation, colour correction and noise suppression. Imaging devices such as pocket cameras, smart phones and professional high-end equipments are common in today's society. Independent of the imaging device, whether or not the user is aware of it, these all require noise suppression in the image acquisition pipeline. Thus, image denoising has a long history in the imaging community and therefore the topic is also a well studied area of research with many practical applications.

Despite well developed theory and the existence of a vast amount of denoising approaches, open problems still remain to be answered. This thesis bridges the gap 
between variational formulations, tensor-based approaches and prior information in an image diffusion framework. The following statement summarises the core contribution of this thesis:

- This thesis explores the effects of introducing contextual information as prior knowledge for image denoising.

The keyword in the above statement is contextual information. To have a context implies that there is an environment of predefined constraints, e.g., there is a "bigger picture". In particular, the aim is to benefit from natural constraints not before exploited for image enhancement and denoising. This approach is expected to achieve better result compared to not using contextual information. The constraints that are introduced should not be image specific, but generic enough to include a large class of images.

This dissertation builds on the vast theory of partial differential equations (PDEs). The PDE framework is perhaps one of the most agile and practical mathematical tools that exists in today's modern science and is often used to describe physical processes.

The main inspiration of this work originates from the physical process of "mass transportation" (or heat conductivity) based on the theory of Fick's law. The principle of Fick's law states that if there is substance in two regions, then if allowed to flow freely, the region with higher concentration will flow to the region of lower concentration, i.e., a directed mass transportation takes place. The problem in image denoising is to introduce mechanisms to control the rate of mass change, such that the image is not distorted. This process is known as diffusion.

The physical interpretation the diffusion process in image processing is that image structures can be used to guide the transportation of mass. This results in an adaptive filtering process which suppresses noise and preserves image features. Thus the aim is to define PDE-based filtering methods that suppress noise but preserve structures such as lines and edges contained in an image.

Figure 1.1 shows some denoising examples using established diffusion methods. Isotropic diffusion corresponds to the "mass transportation"-analogy and, as clearly visible, oversmooths the image. Non-linear and anisotropic diffusion perform better with respect to final image quality than isotropic diffusion since they adapt to the image structure. The adaptation of the non-linear filter is done with respect to the presence of an edge, however the filter does not adapt to change in structure orientation as the anisotropic diffusion. These differences are clearly seen in figure 1.1 the non-linear filter preserves noise close to the image structure whereas the anisotropic filter suppresses noise parallel to the image structure in an orientation dependent way. These diffusion methods are described in chapter 3 Based on this motivation, the topic of this thesis is image diffusion and studies ways of introducing domain-dependent information into the diffusion process. 


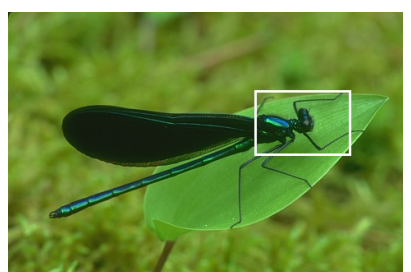

Original

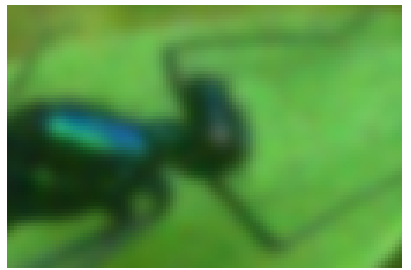

Isotropic diffusion

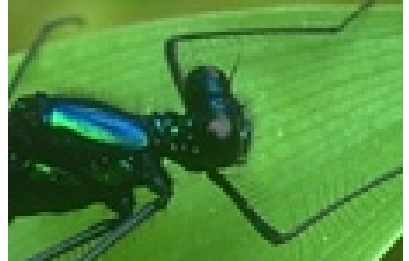

Zoom Original

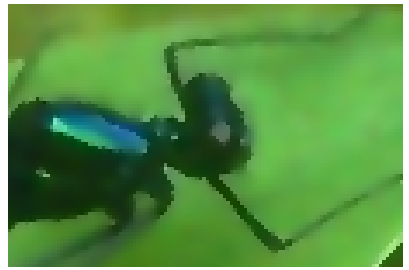

Non-linear diffusion

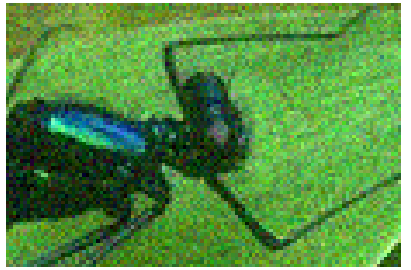

Noisy

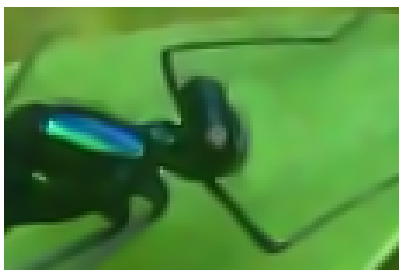

Anisotropic diffusion

Figure 1.1: Given a noisy image, the goal is to remove the noise, but at the same time, preserve important image features such as lines and edges. The second row shows the result of three established diffusion-based denoising methods. These are introduced in chapter 3 . In this example, the original 8 bit colour image was corrupted by 20 standard deviations of additive Gaussian noise. 


\subsection{Organisation of Thesis}

The foundation of the material included in this thesis are parts of several published works. Included publications are presented in the next section which also outlines the abstracts and the authors' contributions to the works. The thesis is organized into five parts, described below. Additionally, figure 1.2 gives an overview of the methods that are included in the thesis and their corresponding chapters.

- Part I presents an overview of the denoising field and introduces notation and terminology that is used throughout the thesis.

Chapter 2 introduces notation, concepts and mathematical tools that have been used in the thesis. Furthermore, the chapter gives an overview and introduction to denoising methods that are relevant to this work. Established denoising methods are grouped with respect to domain definitions and colour extension.

Chapter 3 introduces and derives established diffusion-based denoising methods. The chapter contains explicit derivations of the isotropic and non-linear diffusion-schemes that results in the Euler-Lagrange equations. Also the tensorbased anisotropic diffusion scheme is presented.

- Part II introduces variational image diffusion in non-linear domains.

Chapter 4 presents a novel tensor-based functional that incorporates contextual information of the image domain into its integrand. The functional is studied in great detail and we show that many variational-based denoising methods can be derived from its definition.

Chapter 5 introduces scalar-valued diffusion, such as isotropic and total variation diffusion in non-linear domains as special cases of the generalised formulation in chapter 4

Chapter 6 presents an approach to incorporating locally estimated probability density functions as domain-specific information in the filtering scheme.

Chapter 7 expresses image diffusion in the channel representation for the problem of mixed Gaussian noise distributions and outlier rejection.

- Part III introduces the gradient energy tensor and its applications in computer vision.

Chapter 8 gives the definition of the gradient energy tensor. The tensor is not only used in a theoretical derivation presented in chapter 9 , but also as a substitute for the structure tensor in optical flow and corner detection. Furthermore, chapter 14 introduces fast anisotropic diffusion using the gradient energy tensor.

Chapter 9 introduces the concept of tensor-based total variation using the gradient energy tensor.

- Part IV deals with colour representation, implementational aspects and the evaluation framework for the methods presented in previous parts. 


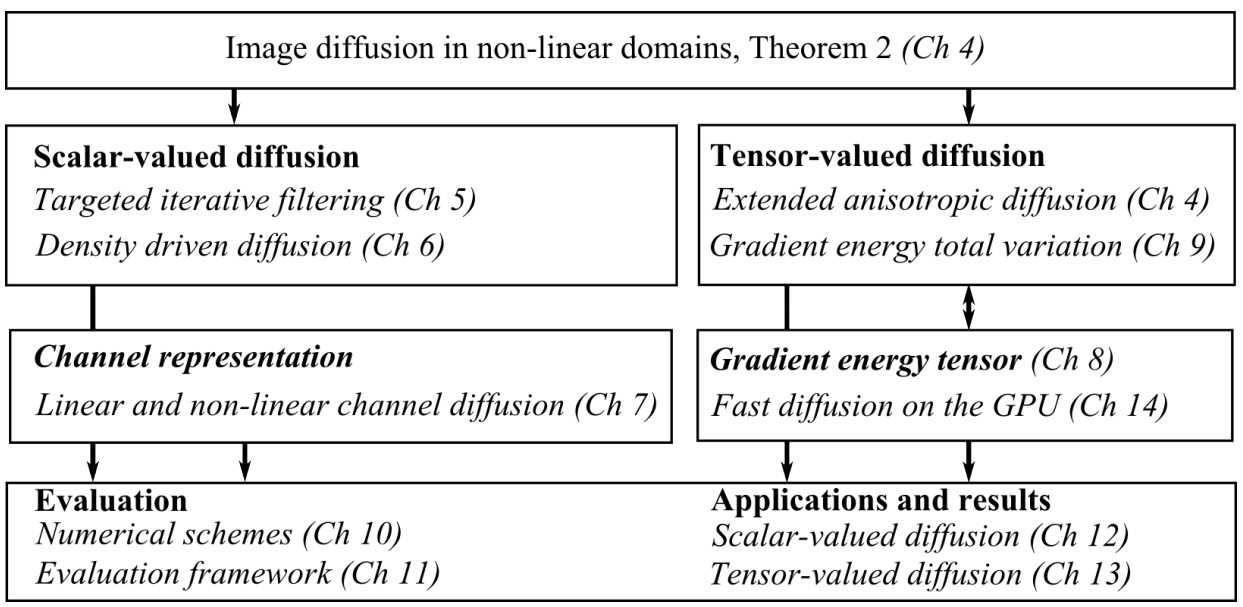

Figure 1.2: Overview of included diffusion methods grouped into scalar and tensorvalued diffusion models with corresponding chapters.

Chapter 10 details the numerical scheme and operators that have been used throughout the evaluation.

Chapter 11 defines the evaluation framework, with respect to colour image representation, image quality metrics and datasets. Additionally, the chapter gives an overview of the compared methods and details the themes of applications evaluated in chapters 12 and 13

Chapter 12 evaluates the scalar-valued formulations presented in chapters 5 , 6 and 7 Applications that are considered include visualization and denoising of computed tomography images, scalar-valued density driven diffusion and channelbased regularization.

Chapter 13 evaluates the tensor-based approaches and includes extended anisotropic diffusion (see chapter 4) and gradient energy total variation (see chapter 9). Furthermore, the chapter presents a hypothesis testing to determine the significance of introducing domain-dependent information into the diffusion schemes.

Chapter 14 defines a diffusion formulation that exploits the gradient energy tensor (chapter 8) to achieve fast anisotropic diffusion on the graphical processing unit.

- Part V summarises the results, suggests directions of future research and open problems.

Chapter 15 summarise and concludes the thesis. 


\title{
1.3 Included publications
}

This section lists the publications included in this thesis. Detailed bibliographic information, abstract and the author's contribution is also given.

\section{I: Color Persistent Anisotropic Diffusion of Images}

F. Åström, M. Felsberg, and R. Lenz. Color Persistent Anisotropic Diffusion of Images. In A. Heyden and F. Kahl, editors, Image Analysis, volume 6688 of Lecture Notes in Computer Science, pages 262-272. Springer, 2011.

\begin{abstract}
:
Techniques from the theory of partial differential equations are often used to design filter methods that are locally adapted to the image structure. These techniques are usually used in the investigation of gray-value images. The extension to color images is non-trivial, where the choice of an appropriate color space is crucial. The RGB color space is often used although it is known that the space of human color perception is best described in terms of non-euclidean geometry, which is fundamentally different from the structure of the RGB space. Instead of the standard RGB space, we use a simple color transformation based on the theory of finite groups. It is shown that this transformation reduces the color artifacts originating from the diffusion processes on RGB images. The developed algorithm is evaluated on a set of real-world images, and it is shown that our approach exhibits fewer color artifacts compared to state-of-the-art techniques. Also, our approach preserves details in the image for a larger number of iterations.
\end{abstract}

\section{Contribution:}

The main novelty in this paper was to suggest a colour model which reduced artefacts introduced when filtering image regions that contain sharp discontinuities in colour intensities. The author contributed to the findings, performed the experiments and the main part of the writing.

\section{II: On Tensor-Based PDEs and their Corresponding Variational For- mulations with Application to Color Image Denoising}

F. Åström, G. Baravdish, and M. Felsberg. On Tensor-Based PDEs and their Corresponding Variational Formulations with Application to Color Image Denoising. In European Conference on Computer Vision (ECCV), volume 7574, pages 215-228, Firenze, 2012. LNCS, Springer Berlin/Heidelberg.

\section{Abstract:}

The case when a partial differential equation (PDE) can be considered as an EulerLagrange (E-L) equation of an energy functional, consisting of a data term and a smoothness term is investigated. We show the necessary conditions for a PDE to be the E-L equation for a corresponding functional. This energy functional 
is applied to a color image denoising problem and it is shown that the method compares favorably to current state-of-the-art colour image denoising techniques.

\title{
Contribution:
}

This paper introduces a novel functional derived from the PDE equations of standard non-linear diffusion schemes. The case when a tensor-based PDE can be expressed as an energy functional is investigated. The author contributed to the derivation of the main theorem, corollary and the proposition. Also the author performed the main part of the writing and performed all experiments.

\section{III: Targeted Iterative Filtering}

F. Åström, M. Felsberg, G. Baravdish, and C. Lundström. Targeted iterative filtering. In A. Kuijper, K. Bredies, T. Pock, and H. Bischof, editors, Scale Space and Variational Methods in Computer Vision (SSVM), volume 7893 of Lecture Notes in Computer Science, pages 1-11. Springer Berlin Heidelberg, 2013.

\begin{abstract}
:
The assessment of image denoising results depends on the respective application area, i.e. image compression, still-image acquisition, and medical images require entirely different behavior of the applied denoising method. In this paper we propose a novel non-linear diffusion scheme that is derived from a linear diffusion process in a value space determined by the application. We show that applicationdriven linear diffusion in the transformed space compares favorably with existing nonlinear diffusion techniques.
\end{abstract}

\section{Contribution:}

This paper is the first paper that introduces a diffusion framework resulting in a non-linear filtering method, which is application-driven rather than data-driven. The author contributed to the findings of the necessary condition for existence of solution, the transformation of statistical moments using a non-linear mapping function, the main part of the writing and performed all experiments.

\section{IV: Density Driven Diffusion}

F. Åström, V. Zografos, and M. Felsberg. Density Driven Diffusion. In 18th Scandinavian Conferences on Image Analysis, 2013, volume 7944 of Lecture Notes in Computer Science, pages 718-730, 2013.

\begin{abstract}
:
In this work we derive a novel density driven diffusion scheme for image enhancement. Our approach, called D3, is a semi-local method that uses an initial structure-preserving oversegmentation step of the input image. Because of this, each segment will approximately conform to a homogeneous region in the image, allowing us to easily estimate parameters of the underlying stochastic process thus achieving adaptive non-linear filtering. Our method is capable of producing
\end{abstract}


competitive results when compared to state-of-the-art methods such as non-local means, BM3D and tensor driven diffusion on both color and grayscale images.

\title{
Contribution:
}

This is the first work that utilized density estimates from an oversegmentation of the image to drive the diffusion process. We used results from our work Targeted Iterative Filtering [7]. The author contributed to the findings and in discussions with co-author V. Zografos, we formulated the presented approach. The experiments were done by the author as well as the method implementation. In particular, V. Zografos was an active contributor to the section "Method Description".

\section{V: Using Channel Representations in Regularization Terms: A Case Study on Image Diffusion}

C. Heinemann, F. Åström, G. Baravdish, K. Krajsek, M. Felsberg, and H. Scharr. Using Channel Representations in Regularization Terms A Case Study on Image Diffusion. Proceedings of the 9th International Conference on Computer Vision Theory and Applications (VISAPP), pages 48-55, 2014.

\begin{abstract}
:
In this work we propose a novel non-linear diffusion filtering approach for images based on their channel representation. To derive the diffusion update scheme we formulate a novel energy functional using a soft-histogram representation of image pixel neighborhoods obtained from the channel encoding. The resulting Euler-Lagrange equation yields a non-linear robust diffusion scheme with additional weighting terms stemming from the channel representation which steer the diffusion process. We apply this novel energy formulation to image reconstruction problems, showing good performance in the presence of mixtures of Gaussian and impulse-like noise, e.g. missing data. In denoising experiments of common scalar-valued images our approach performs competitive compared to other diffusion schemes as well as state-of-the-art denoising methods for the considered noise types.
\end{abstract}

\section{Contribution:}

In this work we present a case study which exploits the channel representation to filter image data containing impulse-like noise. The resulting Euler-Lagrange equations can be interpreted as a derivative of the authors work Targeted Iterative Filtering [7. The two first authors contributed equally to the development of the theory and the method implementation. The author contributed to the experiments and the resulting manuscript.

\section{VI: A Tensor Variational Formulation of Gradient Energy Total Varia- tion}

F. Åström, G. Baravdish, and M. Felsberg. A tensor variational formulation of gradient energy total variation. In X.-C. Tai, E. Bae, T. Chan, 
and M. Lysaker, editors, Energy Minimization Methods in Computer Vision and Pattern Recognition (EMMCVPR), volume 8932 of Lecture Notes in Computer Science, pages 307-320. Springer International Publishing, 2015.

\begin{abstract}
:
We present a novel variational approach to a tensor-based total variation formulation which is called gradient energy total variation, GETV. We introduce the gradient energy tensor [37] into the GETV and show that the corresponding Euler-Lagrange (E-L) equation is a tensor-based partial differential equation of total variation type. Furthermore, we give a proof which shows that GETV is a convex functional. This approach, in contrast to the commonly used structure tensor, enables a formal derivation of the corresponding E-L equation. Experimental results suggest that GETV compares favourably to other state of the art variational denoising methods such as extended anisotropic diffusion (EAD) 2] and total variation (TV) [79] for gray-scale and colour images.
\end{abstract}

\title{
Contribution:
}

This work introduces a tensor-based total-variation based functional and the tensor we consider is the gradient energy tensor. The author contributed to the problem formulation and performed the main part of the derivation. Results on convexity have been achieved based on discussions among all co-authors. The author have done the main part of the manuscript writing, the implementation and the experiments.

\section{VII: On the Choice of Tensor Estimation for Corner Detection, Optical Flow and Denoising}

F. Åström and M. Felsberg. On the Choice of Tensor Estimation for Corner Detection, Optical Flow and Denoising. In Workshop on Emerging Topics in Image Restoration and Enhancement (IREw 2014) in conjunction with Asian Conference on Computer Vision (ACCV) (Accepted), 2014.

\begin{abstract}
:
Many image processing methods such as corner detection, optical flow and iterative enhancement make use of image tensors. Generally, these tensors are estimated using the structure tensor. In this work we show that the gradient energy tensor can be used as an alternative to the structure tensor in several cases. We apply the gradient energy tensor to common image problem applications such as corner detection, optical flow and image enhancement. Our experimental results suggest that the gradient energy tensor enables real-time tensor-based image enhancement using the graphical processing unit (GPU) and we obtain $40 \%$ increase of frame rate without loss of image quality.
\end{abstract}




\title{
Contribution:
}

This is the first work that studies the applicability of replacing the structure tensor with the gradient energy tensor. The main contribution is the GPU implementation, which results in a fast image diffusion framework, done by the author. The implementation, experimental evaluation and the main part of writing was done by the author.

\section{VIII: Domain-Dependent Anisotropic Diffusion}

F. Åström, M. Felsberg, and G. Baravdish. Domain-Dependent Anisotropic Diffusion. Journal of Mathematical Imaging and Vision (submitted), 2014.

\begin{abstract}
:
In this work, we introduce and study a novel functional for image enhancement and denoising. Its regularization term incorporates domain dependent and contextual information using first principles. Few works in literature aim to describe variational models which consider domain dependent information and contextual knowledge of the denoising problem. Our novel functional unifies and extends current state of the art variational formulations. We derive the corresponding Euler-Lagrange equations, using the Gradient Energy Tensor. In contrast to the commonly used structure tensor, we are able to preserve the relation between the variational formulation and the final diffusion equation. Additionally, we present an extensive analysis of properties of the Euler-Lagrange equation, these include: the existence of tensor symmetry constraints, convexity, and geometric interpretation of the proposed domain dependent functional. Lastly, we evaluate the proposed method on the Berkeley colour image dataset, where we define the domain dependent function based on density estimates from an oversegmentation map. We show that incorporating these density estimates into the variational formulation significantly boosts the perceptual quality and reduces error measures of the final results.
\end{abstract}

\section{Contribution:}

This work unifies the author's works II [2], III [7, IV [11] and VI [3] into one coherent framework. The formulation of the main theorem and the derivation of the Euler-Lagrange equation was done by the author. The implementation, evaluation and the main part of writing was done by the author. 


\subsection{Related publications}

The following publications by the author are not included in the thesis.

\section{Journal publications}

G. Baravdish, O. Svensson, and F. Åström. On Backward $p(x)$-Parabolic Equations for Image Enhancement. Numerical Functional Analysis and Optimization, $36(2): 147-168,2015$.

F. Åström and R. Köker. A parallel neural network approach to prediction of Parkinsons Disease. Expert systems with applications, 38(10):12470-12474, 2011.

\section{Other publications}

N. Pettersson and F. Åström. A system for Real-Time Surveillance De-Weathering. In Swedish Symposium on Image Analysis (SSBA), Luleå, 2014.

F. Åström, V. Zografos, and M. Felsberg. Image Denoising via Density Driven Diffusion. In Swedish Symposium on Image Analysis (SSBA), Göteborg, 2013.

F. Åström, M. Felsberg, G. Baravdish, and C. Lundström. Visualization Enhancing Diffusion. In Swedish Symposium on Image Analysis (SSBA), Stockholm, 2012.

F. Åström, M. Felsberg, and R. Lenz. Color Persistent Anisotropic Diffusion of Images. In Swedish Symposium on Image Analysis (SSBA), Linköping, 2011. 


\section{Chapter 2}

\section{Background}

This chapter reviews current state-of-the-art methods for image denoising. As will be seen, extensive research has been performed during the last decades within the field. Naturally, this chapter cannot give a complete overview of current methods due to the large amount of previous works. The chapter aims at providing an introduction to methods that are relevant for this work and to place the contributions of this thesis in a wider context.

There are primarily two classes of successful denoising methods, local and nonlocal methods. In this work we also introduce a third category: the semi-local methods. The primary feature of a non-local method is that it uses self-similarity measures of regions (or patches) in the image data and uses this information to guide the filtering. On the other hand, local methods use local variations, such as directional change, in the image data to find constraints to control the image filtering. Further subdivision can be made, such as if the methods have a natural generalisation to higher-dimensional image data, which is a particularly important feature in colour image denoising.

In the following subsections, we first introduce established denoising algorithms for each method category. Then, concepts and mathematical tools that are used throughout the thesis follows. The final part of this chapter gives additional remarks on concepts related to image diffusion methods.

\subsection{Denoising methods in image processing}

Given an image acquisition system that is modelled using the uncertainty principle, there is a tradeoff between data accuracy and spatial image resolution [32, 43]. This means that it is only possible to have infinite spatial accuracy if the data resolution tends towards zero, i.e., all measured data are just noise. In practice, spatial resolution is limited and thus image data is noisy information.

Estimation of the noise distribution from a noisy signal is an ill-posed problem, see [85] and section 2.2.3. The challenge of image denoising is to compensate for the 
noise and preserve lines and edges to achieve robust filtering. In image processing it is commonly accepted, by the principles of ergodicity and stationarity, that an image pixel value can be assumed to belong to the same stochastic process as its local neighbourhood. Within this statistical interpretation of image representation the semi-local paradigm unifies the local and non-local approaches. The following sections first introduce the local methods before proceeding toward non-local and semi-local formulations.

\subsubsection{Overview}

Table 2.1 lists established methods according to year, type (local, semi-local, and non-local) and if there exists a generalisation to vector-valued images. From the table, the trend of research is obvious; initially researchers were considering local methods, probably since they originate from a rich mathematical theory and many results in mathematics could be used in the context of engineering applications, e.g., image denoising. However, towards the end of the 20th century, the non-local methods garnered a widespread interest in the community due to their excellent performance in visual perception and computational efficiency. A natural extension of the local and non-local paradigm, considered in this thesis, is to move towards semi-local methods. The semi-local formulations attempts to alleviate the drawbacks of purely non-local approaches. One apparent drawback is the difficulty of finding enough similar (local) regions for certain non-repetitive image structures. This can cause, e.g., branches in natural images to be oversmoothed. This is a drawback not present in the local methods. On the other hand, non-local methods are more suitable for denoising if the noise-level is high, simply due to having more observations to better estimate the true image signal.

\subsubsection{Local methods}

Table 2.1 gives an overview of notable denoising methods that have been introduced during the last decades. One of the most simple denoising approaches is to convolve the image with a smooth kernel such as a Gaussian function [50]. The convolution by a Gaussian function was independently discovered and later denoted as linear diffusion [57. The width of a Gaussian function is defined by its standard deviation and should reflect the image noise level. Here, we denote the linear diffusion model as a local filter since it does not consider the image domain to any particular extent. As a direct extension of the linear diffusion model, Perona and Malik 72 presented a non-linear filter that exhibited (at the time) remarkable edge retention, however the method suffers from noise preservation close to edges and lines. In an attempt to reduce the noise-preservation effect, Rudin, Osher and Fatemi [79] minimized the total variation measure in the image. The drawback of the total variation formulation is that it enforces piecewise smooth surfaces in the image plane, and thus the final result may look unnatural. A solution to relax the total variation measure was to consider higher-order differentials. This approach is today known as total generalised variation [20]. Weickert [95] extended the Perona and Malik formulation to include a tensor, known as the diffusion tensor, to steer 


\begin{tabular}{llll}
\hline Method & Year & Type & Colour \\
\hline Gaussian filter [50] & 1959 & local & no \\
Linear diffusion [57] & 1984 & local & no \\
Non-linear diffusion [72] & 1990 & local & no \\
Total variation [79] & 1992 & local & no \\
Anisotropic diffusion [95] & 1998 & local & no \\
Bilateral filtering [90] & 1998 & local & no \\
Beltrami flow [54] & 2000 & local & yes \\
Mean shift denoising [26] & 2002 & non-local & yes \\
Vector-valued PDE [91] & 2003 & local & yes \\
Non-local means [22] & 2005 & non-local & yes \\
Channel smoothing [34] & 2006 & local & no \\
BM3D [27] & 2006 & non-local & no \\
Colour BM3D [28] & 2007 & non-local & yes \\
Total generalized variation 20] & 2009 & local & yes \\
Domain-dependent anisotropic diffusion* & 2015 & semi-local & yes \\
\hline
\end{tabular}

Table 2.1: An overview of established denoising methods that have gained recognition in the image processing community. *Proposed in this thesis.

the filter parallel to the image edges. Another recent approach is our framework, the extended anisotropic diffusion (EAD) [2], which is also a local method that estimates the orientation of image structures using a diffusion tensor. The difference between EAD and anisotropic diffusion is that EAD models a non-symmetric tensor in addition to the standard diffusion tensor.

One local method that induced great interest in the research community is the method called bilateral filtering [90. It is an edge-preserving filter that, in contrast to many denoising methods, is non-iterative. The basic principle of the filter is to estimate a similarity measure, both in the sense of geometric closeness and in photometric closeness, i.e., the filter exploits both domain and range similarity functions. Based on the ideas of bilateral filtering sprung a new paradigm that includes the non-local methods.

\subsubsection{Non-local methods}

An early extension of the bilateral filter, is the non-local means (NLM) filter [22, one of the currently most notable non-local denoising algorithms. The basic approach of NLM is to compute averages of similar image patches in a neighbourhood. The rationale is that if many similar patches in the domain are found (according to some criteria) the image structure is well represented. The patches can be located either in the image domain or, for reasons of computational efficiency, restricted to local regions, i.e., a semi-local formulation. The result of the NLM-algorithm relies on the accuracy of the patch similarity estimate. The standard formulation of the NLM filter 22] considers a photometric and geometrical closeness, similarly 
to bilateral filtering, but with the difference of being patch-based. Several extensions have been made to the NLM filter to include other spatial and photometric similarity measures e.g., [99].

One recognised extension of NLM is "Block-Matching via sparse 3D representation" (BM3D) [28, 27. BM3D computes the similarity between different neighbourhoods to obtain a group of similar image patches. It considers both a non-linear threshold operation as well as a linear Wiener filter approach to stack patches that locally describe the same image region. This stack of patches produce a sparse 3D representation on which weighted filtering can be performed.

The mean shift denoising method (MS) [26] also belongs to the category of non-local filters. MS estimates kernel density functions in a feature space. By modifying the statistical moments of these densities, the MS filter can either be an edge preserving denoising method or be used as a segmentation method.

\subsubsection{Semi-local methods}

Under a statistical motivation and in contrast to the other methods, one major contribution of this thesis is to introduce a novel domain-dependent tensor-based filtering method. It can, for example, be used to utilise probability density estimates to drive the filtering process. These density estimates are probability density functions computed from local segments described by features such as texture or intensities to achieve robust image filtering. In this setting the method consists of three components. The first part involves generating a structure preserving segmentation map by applying an oversegmentation process to each image. Such a map allows for simple, unimodal density models to be easily estimated from the homogeneous information that will be contained in each segment. The second part involves extracting density functions from the segmentation map and the third part minimizes the proposed energy functional, thus achieving a semi-local non-linear adaptive filtering scheme. Therefore, one of the main contributions is to incorporate density and contextual information into an energy functional, resulting in an adaptive filtering scheme based on a stochastic image representation.

\subsection{Preliminaries}

This section gives an overview of important concepts and notation used in the thesis. An effort has been made to maintain a clean and simple notation, thus it should be easy to follow for anyone with a background in the image diffusion community. Therefore, readers familiar with variational calculus, signal processing and image diffusion can in principle skip this section, since it introduces basic concepts found in standard reference works such as [95] and [43].

\subsubsection{Basic notation}

Let the (image) domain be defined by $\Omega \subset \mathbb{R}^{n}$ where $n$ is the dimensionality. Let $u$ denote the image value such that $u\left(x_{1}, \ldots x_{n}\right): \Omega \rightarrow \mathbb{R}$. Often, $n=2$ and the notation $u\left(x_{1}, x_{2}\right)$ means that $x_{1}$ is defined in the horizontal " $x$ "-direction and $x_{2}$ 
in the vertical " $y$ "-direction. Often the dependency on the independent variables will be left out to improve clarity of the presentation, e.g., $u=u\left(x_{1}, \ldots, x_{n}\right)$. Also, note that in general, the results presented in this thesis are valid for $n \geq 2$ if not otherwise stated.

\subsubsection{Noise model}

The image noise that we consider, unless stated otherwise, is assumed to be normal, independent and identically distributed. This means that the image signal can be described by a linear model, where the noise is described by an additive component $\eta$, i.e.,

$$
u_{0}=u+\eta
$$

where $u_{0}$ is the observed signal and $u$ is the noise-free signal. The noise component $\eta$ is modelled as a normal (Gaussian) distribution, i.e.,

$$
f(x, \mu, \sigma)=\frac{1}{\sqrt{2 \pi} \sigma} e^{-\frac{(x-\mu)^{2}}{2 \sigma^{2}}},
$$

such that $\eta \sim \mathcal{N}\left(\mu, \sigma^{2}\right)$ where $\mathbb{E}[\eta]=\mu$ is the mean value and $\operatorname{Var}(\eta)=\sigma^{2}$ is the variance.

\subsubsection{Direct and inverse problem}

There are two types of problems, direct problems and inverse problems. The direct problem is when we have some data (or information) and process it given a known algorithm. One real-life example of a direct problem is cooking: we have the ingredients (the data) and follow a recipe (the algorithm), then the final product is a meal. The inverse problem is then: given a meal, find out what ingredients were used and what was the recipe (algorithm), i.e., find the inverse algorithm. Formally, the above example can be described as follows.

Let $u$ represent the data (ingredients), $A$ represents the algorithm (recipe) and $u_{0}$ the outcome (meal), then

$$
u_{0}=A(u) .
$$

In other words, there is a cause and an effect. The relation between these concepts is illustrated in figure 2.1 .

Given a noisy image $u_{0}$, we formulate models $A(u)$ to recover $u$. $A(u)$ is often an integral operator expressed using derivatives, thus to compute its inverse is an ill-posed problem. According to Hadamard [45, a problem is well-posed if the following conditions are meet

1. Existence: A solution exists.

2. Uniqueness: The solution is unique.

3. Stability: The solution should depend continuously on the input data. 


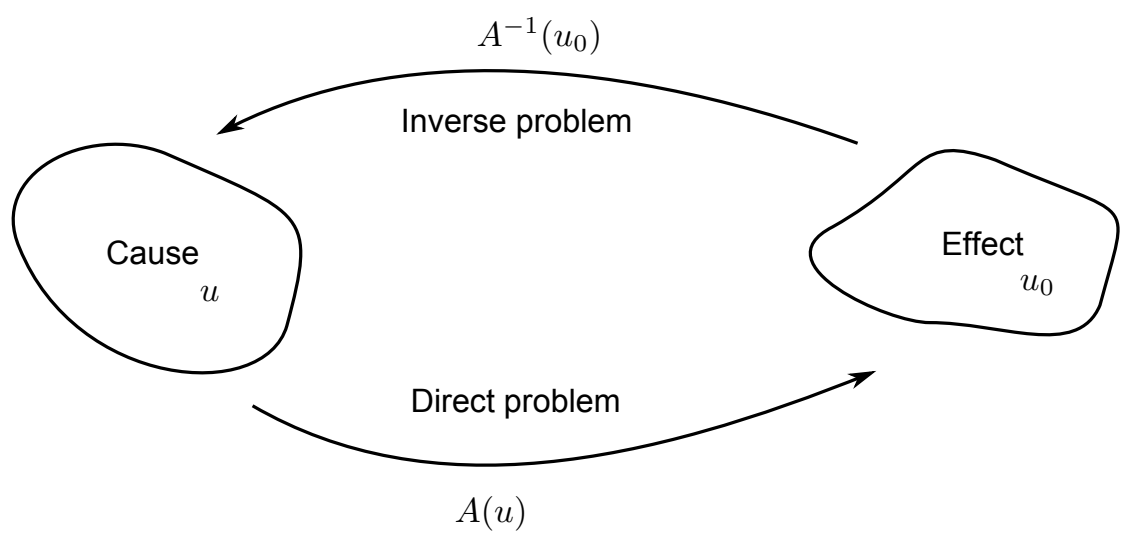

Figure 2.1: Relation direct and inverse problem.

The last condition means that if the input data is perturbed by noise, then the new solution should only be a small modification to the previous solution. If any of the above conditions is not satisfied, then the problem is ill-posed and may require additional regularization or constraints to be solved.

Energy minimization methods are often used to impose well-posedness in illposed problem. The common approach is to formulate and minimize energy functions $E(u)$, i.e.,

$$
u^{*}=\min _{u} E(u)
$$

Moreover, this thesis considers Tikhonov regularization of the following type

$$
E(u)=\int_{\Omega}\left(u-u_{0}\right)^{2} d x+\lambda R(u),
$$

where $R(u)$ is a smoothness term that impose smoothness constraints on the solution $u^{*}$ [88. The parameter $\lambda>0$ determines the influence of the regularizer $R(u)$. The first integral in $(2.5)$ is the data term determining the closeness of $u^{*}$ to the given data $u_{0}$.

\subsubsection{Partial derivatives}

The definition of a partial derivative of the function $u(x)$ with respect to $x$ is

$$
\frac{\partial}{\partial x_{i}} u(x)=\lim _{\varepsilon \rightarrow 0} \frac{u\left(x_{i}+\varepsilon\right)-u\left(x_{i}\right)}{\varepsilon} .
$$

The short-hand notation

$$
u_{x_{i}}=\partial_{x_{i}} u=\frac{\partial}{\partial x_{i}} u(x),
$$

is frequently used to denote differentiation. When writing subscript " $x_{i}$ " it should be understood that $u$ is differentiated with respect to the $x_{i}$-component. When 
the partial derivatives of $u$ are expressed in vector notation, the vector will be denoted as $\nabla u$, e.g.,

$$
\nabla u=\left(\begin{array}{c}
u_{x_{1}} \\
\vdots \\
u_{x_{n}}
\end{array}\right) .
$$

The divergence operator on the gradient field $\nabla u$ results in the following expressions

$$
\operatorname{div}(\nabla u)=\sum_{i=1}^{n} \frac{\partial^{2} u}{\partial x_{i}^{2}}
$$

and

$$
\Delta u=\operatorname{div}(\nabla u)
$$

where $\Delta u$ is the Laplace operator. Intuitively, the difference between the gradient and divergence operators can be explained as follows. The gradient is a vector describing direction and magnitude changes whereas the divergence describes the amount of change.

\subsubsection{Scalar product}

The scalar product is denoted as

$$
s=\nabla u \cdot \nabla v=\nabla^{t} u \nabla v
$$

Furthermore, it is trusted that the reader can, depending on the context, differentiate between, e.g., $n$ being a vector and a scalar.

\subsubsection{Variational formulation}

An integral part of this work is to minimize energy functionals of the type

$$
\min _{u} E(u), \quad \text { where } E(u)=\int_{\Omega} L(u) d x,
$$

and $L(x)$ is the Lagrangian. The function $E(u)$ will be referred to as "energy functional" or "functional", and it is minimized by solving the corresponding EulerLagrange (E-L) equation

$$
\frac{\partial E(u)}{\partial u}=0
$$

with a corresponding boundary condition. The E-L equation is obtained by computing the Gâteaux derivative introduced in the below subsection. If the E-L equation is solved, then the solution is the stationary point such that $E(u)$ has its minimum value. Note that for a unique minimum of $E(u)$ to exist, it is necessary that $L(u)$ is a strict convex function in its argument. 


\subsubsection{Functional derivatives}

The functional derivative of $E(u)$ with respect to $u$ is computed with the Gâteaux derivative defined as

$$
\frac{\partial E(u)}{\partial u} v=\lim _{\varepsilon \rightarrow 0} \frac{E(u+\varepsilon v)-E(u)}{\varepsilon}=\left.\frac{\partial}{\partial \varepsilon} E(u+\varepsilon v)\right|_{\varepsilon=0},
$$

where $v \in \mathcal{C}^{1}(\Omega)$ is an arbitrary function not equal to zero. Additionally, the short notation for functional derivatives is defined as

$$
\frac{\partial E(u)}{\partial u} v=\partial_{u} E(u) v
$$

and is in analogy to the notation used for partial derivatives in section 2.2.4.

In general, when computing the Gâteaux derivative of $E(u)$, a useful tool is Green's first identity derived from the Divergence theorem [85. The identity describes partial integration in higher dimensions, and is defined as

$$
\int_{\partial \Omega} v \frac{\partial u}{\partial n} d S=\int_{\Omega} \nabla v \cdot \nabla u d x+\int_{\Omega} v \Delta u d x,
$$

where $\partial \Omega$ is the boundary element, $d S$ is the bounding surface of the domain $\Omega$ and $n$ is the normal vector directed outwards from the image domain. The derivative $\frac{\partial u}{\partial n}=\nabla u \cdot n$ describes the outward normal direction from the boundary and gives the boundary condition. In many situations it is reasonable to assume no mass-transportation over the domain boundaries. This is modelled by putting $\nabla u \cdot n=0$, also known as the Neumann boundary condition.

\subsubsection{Eigenvalue decomposition}

The eigendecomposition described in this part can be found in any standard reference textbook in calculus and linear algebra, e.g., 49. However, for the sake of completeness, and since the eigenvalue decomposition is an integral part of the methods used in this thesis, the standard results are reiterated.

Let $A \in \mathbb{R}^{n \times n}$ symmetric matrix, then the eigendecomposition is given in the below result.

Theorem 1 (Spectral theorem). Let $A \in \mathbb{R}^{n \times n}$ be a symmetric matrix. Then the decomposition

$$
A=U \Lambda U^{t}
$$

is the eigenvalue decomposition where $U$ denote the eigenvectors and $\lambda_{i}, i=$ $1, \ldots, n$ the eigenvalues on the diagonal of $\Lambda$.

For instance, let $A \in \mathbb{R}^{2 \times 2}$ be a symmetric matrix. Let $A$ be composed of the elements $a, b, c \in \mathbb{R}$ :

$$
A=\left(\begin{array}{ll}
a & b \\
b & c
\end{array}\right),
$$


then according to the spectral theorem (see Theorem 1) there always exists an eigenvector $v$ and an eigenvalue $\lambda$ such that the decomposition

$$
A v=\lambda v, \quad v=\left(\begin{array}{l}
v_{1} \\
v_{2}
\end{array}\right) .
$$

is well-defined. Let $\operatorname{det}(A)=a c-b^{2}$ and $\operatorname{tr}(A)=a+c$, where det is the determinant and $\operatorname{tr}$ is the trace. Then the characteristic equation $\operatorname{det}(A-\lambda I)=0$ results in the eigenvalues

$$
\begin{aligned}
\lambda_{1,2} & =\frac{1}{2}\left(\operatorname{tr}(A) \pm \sqrt{\operatorname{tr}(A)^{2}-4 \operatorname{det}(A)}\right) \\
& =\frac{1}{2}\left(a+c \pm \sqrt{(a-c)^{2}+4 b^{2}}\right),
\end{aligned}
$$

and $\lambda_{1}$ is chosen such that $\lambda_{1}>\lambda_{2}$. The eigenvector $v=\left(v_{1}, v_{2}\right)^{t}$ associated with $\lambda_{1}$ is then obtained by solving the equation system

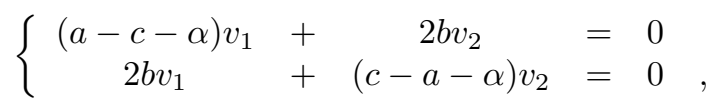

where $\alpha=\sqrt{(a-c)^{2}+4 b^{2}}$. This gives the eigenvectors $v$ and $w$ such that

$$
v \sim\left(\begin{array}{l}
v_{1} \\
v_{2}
\end{array}\right)=\left(\begin{array}{c}
2 b \\
c-a+\alpha
\end{array}\right) \quad \text { and } \quad w \perp v,
$$

where $w$ is the eigenvector corresponding to $\lambda_{2}$. The normalization to unit length has been left out.

\section{Simplified decomposition}

If we do not require an explicit eigenvector representation we can use a simplified eigendecomposition. The decomposition can, for example, be used when forming the diffusion tensor from the structure tensor, concepts introduced in below section 2.2.11. An equivalent reformulation of the eigendecomposition 2.17) for $A \in \mathbb{R}^{2 \times 2}$ is given by

$$
A=U \Lambda U^{t}=\lambda_{1} v v^{t}+\lambda_{2} w w^{t}
$$

where $v, w$ are the two orthonormal eigenvectors in $U$. Now, observe that

$$
v v^{t}+w w^{t}=I \Longleftrightarrow w w^{t}=I-v v^{t},
$$

where $I$ is the identity matrix. Then $A$ can be expressed as 43 ]

$$
A=\left(\lambda_{1}-\lambda_{2}\right) v v^{t}+\lambda_{2} I
$$

and we compute the eigenvector-product $v v^{t}$ as

$$
v v^{t}=\frac{1}{\left(\lambda_{1}-\lambda_{2}\right)}\left(A-\mu_{2} I\right) .
$$

Geometrically, the eigenvalues describe the magnitude of directional change and the corresponding eigenvectors describe the orientation. 


\subsubsection{Taylor series}

The Taylor series of a function $f(x) \in \mathcal{C}^{\infty}$ at a point $a$ is given by

$$
f(x)=\sum_{k=0}^{\infty} \frac{f^{(k)}(a)}{k !}(x-a)^{k}
$$

and $f^{(k)}$ is the $k^{\prime}$ th derivative of $f(x)$.

\subsubsection{Matrix exponential}

A frequently occurring mathematical operation in this thesis is the matrix exponential, and there are many ways to compute it 69 . Let $A \in \mathbb{R}^{n \times n}$, then the matrix exponential of $A$ is given by the power series

$$
e^{A}=\sum_{k=0}^{\infty} \frac{1}{k !} A^{k}
$$

From the eigendecomposition in section 2.2.8, and $A$ is a symmetric matrix with eigenvalues $\lambda_{i} \in \mathbb{R}, i=1 \ldots n$ such that $A=U \Lambda U^{t}$. Then the matrix exponential can be expressed as

$$
e^{A}=\sum_{k=0}^{\infty} \frac{1}{k !} U \Lambda^{k} U^{t}=U\left(\sum_{k=0}^{\infty} \frac{1}{k !} \Lambda^{k}\right) U^{t}=U\left(\begin{array}{ccc}
e^{\lambda_{1}} & & 0 \\
& \ddots & \\
0 & & e^{\lambda_{n}}
\end{array}\right) U^{t}
$$

since $U U^{t}=I$. If $A$ is an arbitrary matrix, then $\lambda_{i} \in \mathbb{C}$ in general. Often we write $\Psi(A)$ where $\Psi(A): \mathbb{R}^{n \times n} \rightarrow \mathbb{R}^{n \times n}$, and should be interpreted as a transformation of $A$ 's eigenvalues such that

$$
\Psi(A)=U\left(\begin{array}{ccc}
\Psi\left(\lambda_{1}\right) & & 0 \\
& \ddots & \\
0 & & \Psi\left(\lambda_{n}\right)
\end{array}\right) U^{t}
$$

The transformation $\Psi(A)$ can also be expressed using the simplified eigendecomposition described in section 2.2.8. Let

$$
\Psi(A)=\Psi\left(\lambda_{1}\right) v v^{t}+\Psi\left(\lambda_{2}\right) w w^{t}
$$

and by substituting 2.26 into 2.31, $\Psi(A)$ can be computed directly as

$$
\Psi(A)=\left[\frac{\Psi\left(\lambda_{1}\right)-\Psi\left(\lambda_{2}\right)}{\lambda_{1}-\lambda_{2}}\right]\left(A-\lambda_{2} I\right)+\Psi\left(\lambda_{2}\right) I .
$$




\subsubsection{Structure tensor}

A central theme in this thesis is the concept of a tensor, specifically we consider the structure tensor [16, 39, 95]. If the image domain is 2-dimensional then the structure tensor is defined as

$$
T(\nabla u)=\left(\begin{array}{cc}
\int_{\Omega} w(x-s) u_{x}(s)^{2} d s & \int_{\Omega} w(x-s) u_{x}(s) u_{y}(s) d s \\
\int_{\Omega} w(x-s) u_{x}(s) u_{y}(s) d s & \int_{\Omega} w(x-s) u_{y}(s)^{2} d s
\end{array}\right),
$$

where $w$ is a smooth kernel, typically the Gaussian function (2.2). In this thesis, the shorthand notation

$$
T(\nabla u)=w *\left(\nabla u \nabla^{t} u\right),
$$

will often be used as a replacement for 2.33. $T$ describes a second moment matrix [40, similarly to a covariance matrix and it is computed by integrating the image gradient over the domain of $u$. If $w$ is selected as

$$
w(x)=\delta(x)= \begin{cases}1, & x=0 \\ 0, & \text { otherwise }\end{cases}
$$

then $T$ is a tensor of rank 1 with eigenvalues $\lambda_{1}=|\nabla u|^{2}$ and $\lambda_{2}=0$ computed using 2.20). If the tensor is of rank 1 then it represents an intrinsically 1-dimensional structure, i.e., it represents an edge [60, 43]. Geometrically this means that the tensor only expresses change orthogonal to the image structure [33. If the two eigenvalues both are non-zero then the image structure represents an intrinsically 2-dimensional signal, which can for example be a corner point. A homogeneous region of the image data is intrinsically 0 -dimensional, this means that both eigenvalues are zero.

\subsubsection{Channel representation}

This subsection introduces the channel representations as an approximative density estimator as described in 34. Channel representations are basically softhistograms, i.e., histograms where samples are not exclusively pooled to the closest bin centre, but to several bins with a weight depending on the distance to the respective bin centre. The "bins" are called "channels". The binning operator or density mapping function is called a basis function, $B(u)$, and is usually nonnegative (as densities are non-negative), has compact support and is smooth (for stability reasons). The measure induced by the mapping to channel representations should be position independent in order to avoid an unwanted bias.

One selection of the basis function is the quadratic B-splines basis function

$$
B(u)= \begin{cases}\frac{3}{4}-u^{2} & 0 \leq|u| \leq \frac{1}{2} \\ \frac{1}{2}\left(\frac{3}{4}-|u|\right)^{2} & \frac{1}{2}<|u| \leq \frac{3}{2} \\ 0 & \text { Otherwise }\end{cases}
$$




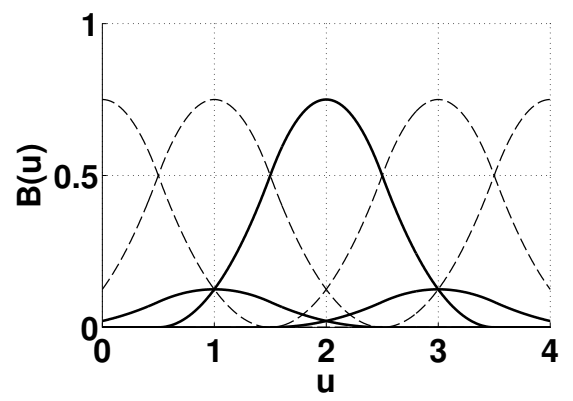

Figure 2.2: Example of 5 equidistantly distributed B-Spline basis functions illustrated with dashed lines. The thick lines represent the encoding of the value 2 from the image domain. In this representation, each image value located on a channel mode is encoded by three basis functions.

Without loss of generality it is assumed that $u(x) \in[0, N-1]$ before the signal can be encoded into $N$ channels. Otherwise $u$ can be transformed linearly to that interval. Figure 2.2 depicts the basis functions in the case of 5 channels. Let $c \in \mathbb{R}^{n}$, be an equidistantly distributed grid over the image range of $u$. Let $c=\left(c_{1}, \ldots, c_{n}\right)^{t}$ be the channel centre vector. Furthermore, the quadratic B-Spline channel representation is denoted as $B=\left(B_{1}, \ldots, B_{N}\right), i=1, \ldots, N$ and is called a channel vector.

The channel representation [42] is a special, lossless soft-histogram, in contrast to other histograms. After encoding a single value in a channel representation, the value can be accurately reconstructed. Using the channel representation allows for simple outlier-removing smoothing, the methods is known as channel smoothing 34] reviewed in section 7.2. Essentially, for each pixel encoding, the values of its local neighbourhood is decoded from the dominant mode to it, i.e., the sub- "bin"position of the maximum value in the soft-histogram.

\subsection{Additional remarks}

In addition to the previously mentioned concepts, the reader should be aware of the following remarks.

\subsubsection{Diffusion and regularization}

Image regularization and image diffusion are closely related. Image regularization is a boundary value problem, equivalent to heat diffusion with source terms in physics. Image diffusion is equivalent to heat diffusion without sources, formulated as an initial value problem, starting with the measured noisy image. In both cases, the regularization term defines the smoothing process leading to noise suppression. For simplicity, we call this process diffusion in both cases, as is usually done in physics. For details of the very close relation between regularization and diffusion see [81]. 


\subsubsection{Continuous representation and discrete solutions}

The methods developed in this thesis are derived using a continuous signal representation. However, in practice, the image data is represented by discrete image samples (pixels), thus there will be a discrepancy between the derived methods and the numerical solutions. Obviously, this discrepancy can be seen as a severe limitation of this work for practical applications. Nevertheless, simple numerical approximations of the introduced methods can in many cases give satisfactory results, though more accurate discretisation schemes would improve the final results. Furthermore, the continuous signal representation allows the use of tools from mathematics not otherwise possible, e.g., the theory of calculus of variations and the framework of partial differential equations.

\subsubsection{Scale-space representation}

Isotropic diffusion (see section 3.2 is closely related to the diffusion equation

$$
\partial_{t} u=\frac{1}{2} \operatorname{div}(\nabla u)
$$

as discussed in 2.3.1. If the image data is 2-dimensional, then the solution of 2.37) is given by the convolution 63

$$
u(x, y ; t)=u_{0}(x, y) * K(x, y ; t),
$$

where $t>0$ is a scale parameter of the smooth kernel $K$ and $u_{0}$ is the initial data. The parameter $t$ can be thought of as the "evolution time", i.e., by solving (2.37) as an initial value problem, the solution at each time-step is smoother than the previous time-step.

In the original definition of a scale-space, the scale-space representation should respect the properties of causality (not introduce new extrema), homogeneity and isotropy (spatial invariance) [57. In this early definition, the kernel $K(x, y ; t)$ in 2.38 should satisfy the following requirements 63. Firstly, the kernel should obey the semi-group property: $K(x, y ; t) * K(x, y ; s)=K(x, y ; s+t)$. Secondly, the kernel should be symmetric and continuity preserving, and lastly $K \rightarrow \delta$ as $t \rightarrow 0$ where $\delta$ is the Dirac delta function (see (2.35). One such kernel which satisfies the requirements is the Gaussian kernel

$$
K(x, y ; t)=\frac{1}{2 \pi t} e^{-\left(x^{2}+y^{2}\right) / 2 t},
$$

which is also part of the explicit solution of the diffusion equation 2.37 [57.

Existence of a scale-space representation for non-linear diffusion such as 3.13 is not straightforward. However, by relaxing the requirement of invariance it can be shown that non-linear processes, such as the Perona and Malik filter, also can be used to represent a scale-space [72. The tensor-based diffusion scheme (3.18) does not result in a valid scale-space representation since the evolution equation consists of spatially adaptive functions, thus violating the symmetry constraint of $K(\cdot, t)$. The same reasoning holds for the methods introduced in this thesis, therefore we have not attempted to give an interpretation in terms of scale-space analogies. 


\section{Chapter 3}

\section{PDE-based image diffusion}

\subsection{Introduction}

The framework of partial differential equations (PDE) occurs frequently in many problems of computer vision. Many image processing techniques are based on variational formulations in their original formulation, e.g., active contours [52], deblurring [25], optical flow [4], and inpainting [14]. The common denominator for these approaches is that they can be interpreted as the result of energy functionals and the differences lie in the modelling of the problem.

This thesis primarily considers the problem of image denoising, and the aim is to remove artefacts in the image data. Particularly, we are interested in the process known as image diffusion. The term diffusion is used to describe the transportation of mass or heat across, e.g., cell membranes. The diffusion process is described by Fick's law

$$
J=-D \nabla u
$$

where $\nabla u$ refers to the concentration gradient, $J$ is the mass flow and $D$ is a diffusivity constant describing the rate of flow 95. This process continues as long as the concentration gradient is non-zero, which is as long as the substance on each side of the membrane wall is not equally distributed. With the motivation of Fick's law, researchers try to model image restoration using this physical process. The analogy can be explained as follows. To enforce a controlled mass transportation within an image yields a regularization of the image values to attain a state of equilibrium. By this process, artefacts in the image are suppressed. Artefacts describe "unwanted" structure. However, what structures are considered as artefacts are situation dependent and thus will be specified in the accurate context.

In a wider context, the primary motivation is to establish a connection between a variational formulation and a tensor-based PDE scheme, which remains a largely unsolved problem. This would enable the extension of variational methods to include tensor components, possibly further improving the final results independent of application area. 


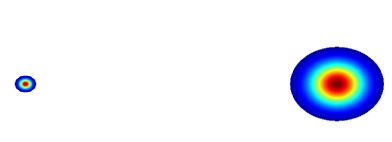

$1 \mathrm{sec}$
$20 \mathrm{sec}$

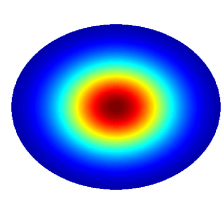

$100 \mathrm{sec}$

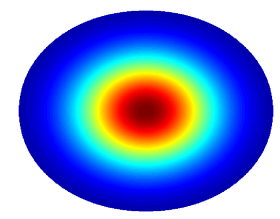

$150 \mathrm{sec}$

Figure 3.1: Evolution of a Gaussian kernel over time.

The remainder of this chapter serves to introduce the isotropic, non-linear and anisotropic diffusion methods. While doing so, the section on isotropic and non-linear diffusion derives the corresponding diffusion schemes in great detail. Furthermore, tensor-based anisotropic diffusion is reviewed in the last section and the possible existence of a variational formulation will be discussed in chapter 4 .

\subsection{Isotropic diffusion}

The variational approach to image diffusion is to model an energy functional $E(u)$ consisting of a data term and a regularization term $R(u)$, i.e.

$$
E(u)=\frac{1}{2} \int_{\Omega}\left(u-u_{0}\right)^{2} d x+\lambda R(u)
$$

where $x \in \Omega$ and $u_{0}$ denotes the observed (noisy) image. The constant $\lambda$ is a positive scalar which determines the amount of the regularization. The domain $\Omega$ is a grid described by the image size in pixels. If

$$
R(u)=\frac{1}{2} \int_{\Omega}|\nabla u|^{2} d x
$$

then $R(u)$ describes linear diffusion, i.e., 3.2 is closely related to the model describing the mass transportation process (3.1), the most fundamental diffusion method.

In order to find the solution $u^{*}$ which minimizes $(3.2)$, one searches for stationary points by computing the Euler-Lagrange (E-L) equation

$$
\frac{\partial E(u)}{\partial u}=0 \quad \text { in } \quad \Omega, \quad \nabla u \cdot n=0 \quad \text { on } \quad \partial \Omega
$$

where $n$ is the normal vector on the boundary $\partial \Omega$, and - denotes the scalar product. The interpretation of the boundary condition is that there is no mass transportation across the boundary resulting in a preservation of the average intensity level of the image. This boundary condition is a Neumann boundary condition.

In order to obtain the E-L equation, compute the Gâteaux derivative us- 
ing 2.14. Then, with the regularization term in 3.3 we obtain

$$
\begin{aligned}
\partial_{u} R v & =\lim _{\varepsilon \rightarrow 0} \frac{1}{2} \frac{1}{\varepsilon} \int_{\Omega}|\nabla(u+\varepsilon v)|^{2}-|\nabla(u)|^{2} d x \\
& =\lim _{\varepsilon \rightarrow 0} \frac{1}{2} \frac{1}{\varepsilon} \int_{\Omega}\left(|\nabla u|^{2}+2 \varepsilon \nabla u^{t} \nabla v+\varepsilon^{2}|\nabla v|^{2}\right)-|\nabla u|^{2} d x \\
& =\int_{\Omega} \nabla u^{t} \nabla v d x,
\end{aligned}
$$

and by applying Green's first identity [85], the above expression result in

$$
\int_{\Omega} \nabla u^{t} \nabla v d x=\int_{\partial \Omega} v(\nabla u \cdot n) d S-\int_{\Omega} v \operatorname{div}(\nabla u) d x .
$$

With Neumann condition $\left.\nabla u \cdot n\right|_{\partial \Omega}=0$, the previous equation reads

$$
\partial_{u} R v=-\int_{\Omega} v \operatorname{div}(\nabla u) d x
$$

From (3.4) and since $v \neq 0$ we obtain the condition that

$$
-\Delta u=-\operatorname{div}(\nabla u)=0
$$

where $\Delta$ is the Laplace operator. Then the $u^{*}$ that minimizes $E(u)$ is obtained by solving the Euler-Lagrange equation

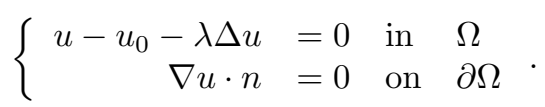

Since $\Delta$ is a rotationally symmetric operator, it does not adapt to any image content such as lines and edges. This results in oversmoothing of image structures. If $(3.9)$ is reformulated into a parabolic form then the solution at a time $t$ has been shown to relate to the framework of scale-space representation 63 , where the finest scale is given at a time $t=0$ and the coarsest scale is obtained at $t \rightarrow \infty$, see section 2.3.3 for an extended discussion. It is often convenient to reformulate PDEs of the form $(3.9)$ to initial value problems. Therefore, in practice, the solution is obtained using an iterative scheme, and is stopped before $t \rightarrow \infty$. Figure 3.1 illustrates the evolution of a Gaussian function as the number of iterations increases over time. Numerical aspects and discretisation of diffusion methods are described in chapter 10.

\subsection{Non-linear diffusion}

This section introduces the concept of non-linear image diffusion, also known as Perona and Malik, or PM, diffusion [72. It is a filtering process designed to avoid some of the drawbacks of isotropic filtering, i.e., that the Gaussian kernel oversmooths details in the image. The novelty in this scheme is to reduce the filtering at image structures with large gradients such as lines and edges. A regularization 
term which models this behaviour, without spatial regularization of the image gradient, is

$$
R(u)=\int_{\Omega} \Phi(|\nabla u|) d x
$$

where $\Phi: \mathbb{R} \rightarrow \mathbb{R}$. In order to minimize $R(u)$, the variational derivative is computed as

$$
\begin{aligned}
\partial_{u} R v & =\left.\int_{\Omega} \frac{\partial}{\partial \varepsilon} \Phi(|\nabla(u+\varepsilon v)|)\right|_{\varepsilon=0} d x \\
& =\left.\int_{\Omega} \frac{\Phi^{\prime}(|\nabla(u+\varepsilon v)|)}{|\nabla(u+\varepsilon v)|} \nabla(u+\varepsilon v) \cdot \nabla v\right|_{\varepsilon=0} d x,
\end{aligned}
$$

which results in

$$
\partial_{u} R v=\int_{\Omega} \frac{\Phi^{\prime}(|\nabla u|)}{|\nabla u|} \nabla u \cdot \nabla v d x .
$$

Using Green's identity, and Neumann boundary condition, the E-L equation is obtained as

$$
\left\{\begin{array}{rlll}
u-u_{0}-\lambda \operatorname{div}(\Psi(|\nabla u|) \nabla u) & =0 & \text { in } \quad \Omega \\
\nabla u \cdot n & =0 & \text { on } & \partial \Omega
\end{array},\right.
$$

where

$$
\Psi(|\nabla u|)=\frac{\Phi^{\prime}(|\nabla u|)}{|\nabla u|} .
$$

If $\Psi$ is chosen as a strictly decreasing function such that if $|\nabla u| \rightarrow 0$ then $\Psi \rightarrow 1$ and if $|\nabla u| \rightarrow \infty$ then $\Psi \rightarrow 0$, one obtains an inhibition of the filtering process for large image gradients. In homogeneous regions the filtering will be isotropic. The function $\Phi$ in $E(u)$ can be obtained by integration of (3.14), (see [2, 17])

$$
\Phi(|\nabla u|)=\int_{\Omega} \Psi(|\nabla u|)|\nabla u| d|\nabla u| .
$$

Two popular selections of diffusivity functions $\Psi$, with the corresponding $\Phi$ function computed according to 3.15 , are

$$
\begin{aligned}
\Psi(|\nabla u|)= & \frac{1}{1+(|\nabla u| / k)^{2}} \\
& \Longrightarrow \Phi(|\nabla u|)=\frac{k^{2}}{2} \log \left(1+\left(\frac{|\nabla u|}{k}\right)^{2}\right)+C,
\end{aligned}
$$

and

$$
\begin{aligned}
\Psi(|\nabla u|)= & \exp \left(-(|\nabla u| / k)^{2}\right) \\
& \Longrightarrow \Phi(|\nabla u|)=-\frac{k^{2}}{2} \exp \left(-(|\nabla u| / k)^{2}\right)+C,
\end{aligned}
$$

where $k$ is an edge-stopping parameter fixed to suppress the flux at edges and lines in the image. The constant $C$ should be chosen such that $\Phi$ is non-negative. 


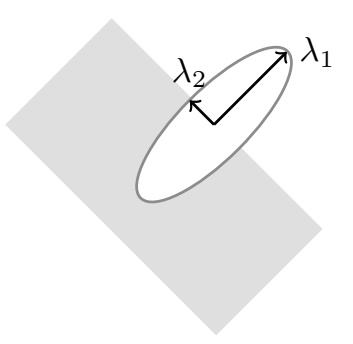

(a) Structure tensor

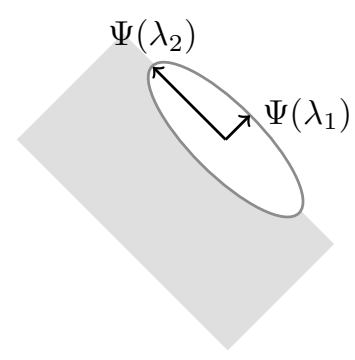

(b) Diffusion tensor

Figure 3.2: Illustration of the structure tensor with eigenvalues $\lambda_{1}$ and $\lambda_{2}$ transformed to align with the image structure.

The parameter $k$ can be selected according to methods such as histogram analysis or decision theory [97, robust statistics [80, or noise estimation methods 33. Black and Rangarajan [17] show that for certain $\Psi$-functions there exists families of corresponding $\Phi$-functions.

\subsection{Tensor-based diffusion}

The non-linear filter by Perona and Malik was an important step in developing robust edge-aware filtering schemes. An equally important step was to introduce an orientation sensitive function, called a tensor, in the divergence form of the PDE. As a result of introducing the tensor, the filtering process is steered parallel to the image structure, preserving edges. The approach was introduced by Weickert and is known as anisotropic diffusion [95.

Before proceeding we want to clarify the terminology is used in this thesis. Some authors denote the diffusion scheme of Perona and Malik as anisotropic diffusion, since it does adapt to the image structure. The terminology is ambiguous since the tensor-based case does also adapt the filtering to the image structure, but in an orientation dependent, i.e., orientation anisotropic way. Therefore, this thesis follows the modern terminology introduced by Weickert [95] and refers to the PM diffusion as "non-linear diffusion" and tensor-based diffusion as "anisotropic diffusion".

The anisotropic diffusion PDE is defined as

$$
\left\{\begin{array}{rlll}
u-u_{0}-\lambda \operatorname{div}(D(T) \nabla u) & =0 & \text { in } & \Omega \\
\nabla u \cdot n & =0 & \text { on } & \partial \Omega
\end{array},\right.
$$

where $D(T)$ denotes the diffusion tensor computed from the structure tensor $T$.

The function $D(T)$ denotes the diffusion tensor and aligns the structure tensor to the image structure, meaning that the eigenvalues of $T$ are remapped using a monotonically decreasing function $\Psi(s)$ such that $\Psi(0)=1$ and $\Psi(s)=0$ as $s \rightarrow$ $\infty$, conditions fulfilled by, e.g., 3.16) or 3.17). Figure 3.2 shows the orientation of the structure tensor in (a) and the diffusion tensor in (b). The same figure 
also illustrates why it is not appropriate to use the structure tensor directly in the diffusion framework. Consider the two principal axes of the structure tensor. The axis with the largest eigenvalue, $\lambda_{1}$, is orthogonal to the image structure. This means that the largest "flow" will be across the image edges, thus the tensor will blur the image data if used directly the filtering process.

Naturally, other $\Psi$-functions than the mentioned ones are possible, e.g., the framework of coherence enhancing diffusion by Weickert 96. produce vivid and visually appealing results.

PDE formulation on the divergence form 3.18 is not the only representation of anisotropic diffusion. An alternative to the divergence form was introduced by Tschumperlé 91] who considered vector-valued PDEs. The framework expresses the PDE (including the diffusion tensor) in one purely isotropic component and one anisotropic component.

Additionally, Felsberg 33 derived an autocorrelation-driven diffusion scheme resulting in the approximation $\operatorname{div}(D) \nabla u \approx 0$ in the expansion

$$
\operatorname{div}(D \nabla u)=\operatorname{div}(D) \nabla u+\operatorname{tr}(D H u),
$$

thus, reducing the complexity of the numerical implementation considerably. The method is denoted as trace-based (TR) diffusion. 


\section{Chapter 4}

\section{Tensor-valued diffusion in non-linear domains}

The linear diffusion scheme (convolution with a Gaussian kernel) is the solution of the diffusion equation, a partial differential equation (PDE), and it is closely related to the notion of scale-space [63, 57]. Therefore it has been of interest to investigate also the Perona and Malik, or PM, formulation and its successors of adaptive image filtering in terms of a variational framework. The motivation is that the variational framework allows for a generic approach to model the denoising problem. By modifying the objective function, this allows existing problem domains to be included, e.g., deconvolution, segmentation and inpainting.

The main contribution of this chapter is the introduction and study of a domain-dependent tensor-driven anisotropic diffusion scheme. We present a regularization term which includes a mapping-function that guides a tensor in the non-linear domain. The mapping-function can be viewed as a domain-dependent transformation of the image data domain. The transformation that is used depends on the specific problem formulation and the desired behaviour of the diffusion scheme. This thesis investigates several options for selecting the mapping function (see chapter 12 and 13) and also investigates properties of the resulting PDE schemes in subsequent chapters.

\subsection{Domain-dependent non-linear transformations}

A simple approach to noise reduction is achieved by applying an isotropic convolution kernel to the image, e.g., normalized Gaussian filters or box filters [57]. Naturally, due to the simplicity of the approach, spatial and contextual information are neglected, and will cause blurring of image structures. As pointed out in the previous chapter, a common approach to adaptive filtering is to let the image gradient control the amount of filtering to improve the image quality.

Naturally, the definition of quality is dependent on the situation where the 
images are used. The focus of this chapter is on denoising algorithms and will concentrate on that noise that actually will be visible to an observer, rather than data noise in general.

Image processing tools that target specific regions of an image are relevant in many areas of computer vision, and include high dynamic range imaging [29, 31, infrared imaging [93] and medical imaging [75]. One such region based diffusion filtering method was proposed by Kačur et al. [53], who generalised the Perona and Malik non-linear diffusion. Kačur et al. model the diffusion PDE with an additional non-linear function on the range domain from which the gradient is computed. This allows the filtering process to be directed to regions containing particular image structures. Their framework reduces the filtering process in regions determined by the user, but the method still requires the determination of a parameter corresponding to an edge-stopping function within the region of filtering.

Rather than modifying the PM PDE-formulation, as Kačur et al. 53] did, this thesis aim at describing a variational model which incorporates the non-linear behaviour into the functional. Before describing the model, consider the difference between linear and non-linear diffusion introduced by Perona and Malik 72 .

The PM scheme extends the linear diffusion scheme which is based on the image gradient $\nabla u$ with an edge stopping function $\Psi(|\nabla u|)$, i.e.

$$
\underbrace{\nabla u}_{\text {Linear }} \rightarrow \underbrace{\Psi(|\nabla u|) \nabla u}_{\text {Perona and Malik }}
$$

where a modification of the diffusion speed is based on the value range of the image gradient. Another PDE model which has received much attention in recent years is the tensor-based diffusion scheme of Weickert 95]. These diffusion models require the determination of parameters often estimated from the input data. Thus the performance of these methods depend on the accuracy of the parameter estimation. A particular problem is that image structure of different scales can be present within the same value ranges, hence spatially varying contrast parameters would be required to tackle the problem of scale.

The interpretation of our approach is that it modifies the value domain of $u$ rather than the gradient domain using a mapping function, $m(u)$, i.e.,

$$
\nabla u \quad \rightarrow \quad \nabla m(u)=m^{\prime}(u) \nabla u .
$$

The energy functional will be modelled in such a way that the regularization term is expressed using the mapping function and the resulting Euler-Lagrange equation can be interpreted in terms of non-linear diffusion in the case of isotropic diffusion. The difference between the edge-stopping function $\Psi$ and the mapping function $m$ is that the former is a data-driven ad-hoc selection whereas $m$ is applicationdriven. Thus an interpretation of how to set the mapping function lies in the definition of prior knowledge of the problem formulation.

The term "prior knowledge" is a general expression and can be interpreted in a wide context. In essence, "prior knowledge" implies some available knowledge that can be used to solve a problem. However, what is "prior knowledge" in the 
context of image enhancement and filtering? To answer this question consider the aim of the filtering process, namely to improve the visual perception of the image. In order to achieve this goal we are required to constrain ourself to consider image features. Properties that are relevant for image enhancement primarily include edges, corners and colour information. Thus, in our context prior information should model be thought of describing relevant features.

The characteristic feature of an edge is that it typically has a large gradient, in contrast to homogeneous regions, which have small gradients. Using this information, i.e., to separate edges from homogeneous regions, will preserve the edge characteristics during the filtering process. Thus the prior knowledge in this example is "preservation of the edge strength will produce perceptually good images".

Before introducing the full tensor-based formulation of the domain-dependent functional, the next section describes the transformation of the noise characteristics under the influence of the mapping function.

\subsection{Noise estimation in the transformed domain}

Transforming the image data value range, does not only modify a region of interest, but also transforms the noise characteristics. Therefore, before proceeding to introduce the domain-dependent diffusion scheme, we investigate the effects of the mapping's first and second statistical moments in the new domain. The idea is to approximate the mapping function using a Taylor expansion, whereby deriving the mean and variances from a second order approximation.

Assuming that the noise can be described as an additive component $\eta$ in $u_{0}=$ $u+\eta$, we consider two cases. Firstly, we consider the transformation of the mean value of $\eta \sim \mathcal{N}\left(\mu, \sigma^{2}\right)$ about $\mu$. In the second step, we analyse the influence of the image data on the noise transformation.

The Taylor expansion (2.27) of only the noise component $\eta$ about $a=\mu$ is given by

$$
m(\eta)=m(\mu+(\eta-\mu))=\sum_{k=0}^{\infty} m^{(k)}(\mu) \frac{(\eta-\mu)^{k}}{k !},
$$

the mean of the second order approximation is then

$$
\begin{aligned}
\mathbb{E}[m(\eta)] & \approx \mathbb{E}\left[m(\mu)+m^{\prime}(\mu)(\eta-\mu)+\frac{1}{2} m^{\prime \prime}(\mu)(\eta-\mu)^{2}\right] \\
& =m(\mu)+\frac{1}{2} m^{\prime \prime}(\mu) \sigma^{2}
\end{aligned}
$$

However, we are not interested in the noise transformation of the single noise component, but in its dependency on the actual data.

Next, consider the statistical moments of the noise under the influence of $u$. In this case the Taylor expansion is

$$
m\left(u_{0}\right)=m(u+\mu+(\eta-\mu))=\sum_{k=0}^{\infty} m^{(k)}(u+\mu) \frac{(\eta-\mu)^{k}}{k !} .
$$


In the following results we consider a second order Taylor series and we start by computing the the mean value:

$$
\begin{aligned}
\mathbb{E}\left[m\left(u_{0}\right)\right] & \approx \mathbb{E}\left[m(u+\mu)+m^{\prime}(u+\mu)(\eta-\mu)+\frac{1}{2} m^{\prime \prime}(u+\mu)(\eta-\mu)^{2}\right] \\
& =m(u+\mu)+\frac{1}{2} m^{\prime \prime}(u+\mu) \sigma^{2},
\end{aligned}
$$

which is similar to 4.4. The transformation of the variance is then

$$
\begin{aligned}
\operatorname{Var}\left(m\left(u_{0}\right)\right) & =\mathbb{E}\left[m\left(u_{0}\right)^{2}\right]-\mathbb{E}\left[m\left(u_{0}\right)\right]^{2} \\
& =\mathbb{E}\left[m(u+\eta)^{2}\right]-\mathbb{E}[m(u+\eta)]^{2} .
\end{aligned}
$$

Below we treat the two expressions of 4.7 separately and we start by expanding $\mathbb{E}\left[m\left(u_{0}\right)^{2}\right]:$

$$
\begin{aligned}
& \mathbb{E}\left[m\left(u_{0}\right)^{2}\right]= \mathbb{E}\left[m(u+\eta)^{2}\right]=\mathbb{E}\left[m(u+\mu+(\eta-\mu))^{2}\right] \\
& \approx \mathbb{E}\left[\left(m(u+\mu)+m^{\prime}(u+\mu)(\eta-\mu)\right)^{2}\right] \\
&=\mathbb{E}\left[m(u+\mu)^{2}+2 m(u+\mu) m^{\prime}(u+\mu)(\eta-\mu)\right. \\
&\left.\left.\quad+m^{\prime}(u+\mu)^{2}(\eta-\mu)^{2}\right)\right] \\
&=m(u+\mu)^{2}+m^{\prime}(u+\mu)^{2} \sigma^{2} .
\end{aligned}
$$

Inserting 4.8 and 4.6 into 4.7 result in the variance

$$
\begin{aligned}
\operatorname{Var}\left(m\left(u_{0}\right)\right)= & \mathbb{E}\left[m\left(u_{0}\right)^{2}\right]-\mathbb{E}\left[m\left(u_{0}\right)\right]^{2} \\
= & m(u+\mu)^{2}+m^{\prime}(u+\mu)^{2} \sigma^{2} \\
& \quad-\left\{m(u+\mu)+\frac{1}{2} m^{\prime \prime}(u+\mu) \sigma^{2}\right\}^{2} \\
= & m(u+\mu)^{2}+m^{\prime}(u+\mu)^{2} \sigma^{2} \\
- & \left\{m(u+\mu)^{2}+m(u+\mu) m^{\prime \prime}(u+\mu) \sigma^{2}+\frac{1}{4} m^{\prime \prime}(u+\mu)^{2} \sigma^{4}\right\} \\
= & m^{\prime}(u+\mu)^{2} \sigma^{2}-m(u+\mu) m^{\prime \prime}(u+\mu) \sigma^{2}-\frac{1}{4} m^{\prime \prime}(u+\mu)^{2} \sigma^{4} \\
= & \left(m^{\prime}(u+\mu)^{2}-m(u+\mu) m^{\prime \prime}(u+\mu)\right) \sigma^{2} \\
& \quad-\frac{1}{4} m^{\prime \prime}(u+\mu)^{2} \sigma^{4} .
\end{aligned}
$$

Hence, an approximation to the transformed mean value and variance is

$$
\begin{aligned}
& \hat{\mu}_{m}=m(u+\mu)+\frac{1}{2} m^{\prime \prime}(u+\mu) \sigma^{2}, \\
& \hat{\sigma}_{m}^{2}=\Psi[m](u+\mu) \sigma^{2},
\end{aligned}
$$

where $\sigma^{4}$ has been neglected in the estimated variance and

$$
\Psi[m](u+\mu)=m^{\prime}(u+\mu)^{2}-m(u+\mu) m^{\prime \prime}(u+\mu),
$$

is the energy operator 36]. Furthermore, we see that the mean value in the transformed domain depends on the curvature of the transformation. This implies that the mapping will in general not preserve the average intensity level. 


\subsection{Tensor-valued domain-dependent functional}

In this part we incorporate a mapping function $m: \mathbb{R} \rightarrow \mathbb{R}$ into the framework of tensor-based image diffusion. We present the functional, derive its E-L equation and investigate its properties. Before proceeding we make the following remarks to emphasise the contribution of this chapter.

The functional unifies standard approaches of image diffusion methods. The included methods are shown in table 4.1. The formulation has the following characteristics

- It is tensor-based

- It incorporates the mapping function

- It is expressed in $u: \mathbb{R}^{n} \rightarrow \mathbb{R}$

Before studying the theorem, we introduce notation and definitions which will simplify the presentation. Let $m \in \mathcal{C}^{2}$ denote the mapping function and $W: \mathbb{R}^{n} \rightarrow$ $\mathbb{R}^{n \times n}$ a tensor and $\nabla m(u) \mapsto W(\nabla m(u))$. Set $\boldsymbol{s}=\nabla m(u)$ such that $W_{s_{i}}(\boldsymbol{s})$ is the component-wise differentiation of $W(s)$ w.r.t. $s_{i}$. Then $W_{\boldsymbol{s}}(\boldsymbol{s}) \in \mathbb{R}^{n^{2} \times n}$ is defined as

$$
W_{\boldsymbol{s}}(\boldsymbol{s})=\left(\begin{array}{c}
W_{s_{1}}(\boldsymbol{s}) \\
\vdots \\
W_{s_{n}}(\boldsymbol{s})
\end{array}\right) .
$$

To further simplify notation in the following calculations we introduce a prescriptnotation for matrix contraction. Define

$$
\nabla u W_{\boldsymbol{s}}(\boldsymbol{s})=\left(\begin{array}{c}
\nabla u^{t} W_{s_{1}}(\boldsymbol{s}) \\
\vdots \\
\nabla u^{t} W_{s_{n}}(\boldsymbol{s})
\end{array}\right),
$$

which contracts $n^{2} \times n$ matrices such as 4.13 to an $n \times n$ matrix.

The theorem below was first introduced in our work [5] and generalises our initial formulation [2] (cf. Theorem 1) to dimensions $n>2$. The theorem also includes the mapping function. Here, we re-state the theorem, introduce newly found identities, which result in a generalised tensor-based domain-dependent image denoising framework.

Theorem 2 (Unifying functional). Let the regularization term $R(u)$, be given by

$$
R(u)=\int_{\Omega} \nabla^{t} m(u) W(\nabla m(u)) \nabla m(u) d x,
$$

where $u, m(u) \in \mathcal{C}^{2}$ and $W: \mathbb{R}^{n} \rightarrow \mathbb{R}^{n \times n}$, and $\nabla m(u) \mapsto W(\nabla m(u))$ is a tensorvalued function. Then the corresponding $E-L$ equation is given by

$$
\left\{\begin{array}{rl}
u-u_{0}-\lambda\left(\left(m^{\prime}\right)^{2} \operatorname{div}\left(S_{1} \nabla u\right)+\operatorname{div}\left(\left(m^{\prime}\right)^{2} S_{2} \nabla u\right)\right) & =0 \text { in } \Omega \\
n \cdot\left(S_{1}+S_{2}\right) \nabla u & =0 \text { on } \partial \Omega
\end{array},\right.
$$


where

$$
\begin{aligned}
& S_{1}=2 W+m^{\prime}{ }_{\nabla u} W_{\boldsymbol{s}}(\boldsymbol{s}), \\
& S_{2}=2 W^{t}+m_{\nabla u}^{\prime} W_{\boldsymbol{s}}(\boldsymbol{s}) .
\end{aligned}
$$

Proof. The proof is given in Appendix A.1.

From theorem 2 we deduce the following properties

1. The E-L equation is made up of two divergence terms, which can be thought of as a linear combination of a non-linear and a linear term. The weight is given by the squared mapping function derivative. If $S_{1}$ and $S_{2}$ are both isotropic tensors, then the divergence-term which corresponds to $S_{1}$ would be the linear term.

2. The tensor $S_{1}$ and $S_{2}$ are different only if $W$ is not symmetric. In the symmetric case, we will see below (Corollary 2), that the E-L equation can be reformulated to a single divergence form.

3. Depending on the choice of the mapping function $m$ and tensor $W$ in 4.15 , table 4.1 shows that the result in Theorem 2 generalises to several diffusion methods from literature.

The subsequent sections shows additional selections of $W$ and $m$. Standard methods such as total variation [2 and isotropic diffusion are extended to include the mapping function. However, before proceeding to specify $W$ and $m$, consider the below properties of the proposed functional and the corresponding E-L equation in Theorem 2,

\subsubsection{Analysis of the Euler-Lagrange equation}

\section{Tensor symmetry}

In general $\nabla_{u} W_{\boldsymbol{s}}(\boldsymbol{s})$ is a non-symmetric tensor, but in certain applications one may be interested in positive definiteness or other symmetry constraints. Therefore, we show in this section sufficient conditions for $W$ to yield a symmetric ${ }_{\nabla u} W_{\boldsymbol{s}}(\boldsymbol{s})$. Based on this motivation, we derive a tensor $S$ such that $S_{1}$ and $S_{2}$ are symmetric, i.e.,

$$
S=S_{1}=S_{2}
$$

It is easy to verify that two additional constraints are required to enable $S$ to be of the above form: it is necessary that $W=W^{t}$ and ${ }_{\nabla u} W_{\boldsymbol{s}}(\boldsymbol{s})=\left({ }_{\nabla u} W_{\boldsymbol{s}}(\boldsymbol{s})\right)^{t}$. The below corollary states the set of symmetric tensors $W$ which yields a symmetric $S$ in 4.19. Let $a, b, c \in \mathbb{R}$ and

$$
W=\left(\begin{array}{ll}
a & b \\
b & c
\end{array}\right),
$$

then the below corollary states the symmetry constraints. 


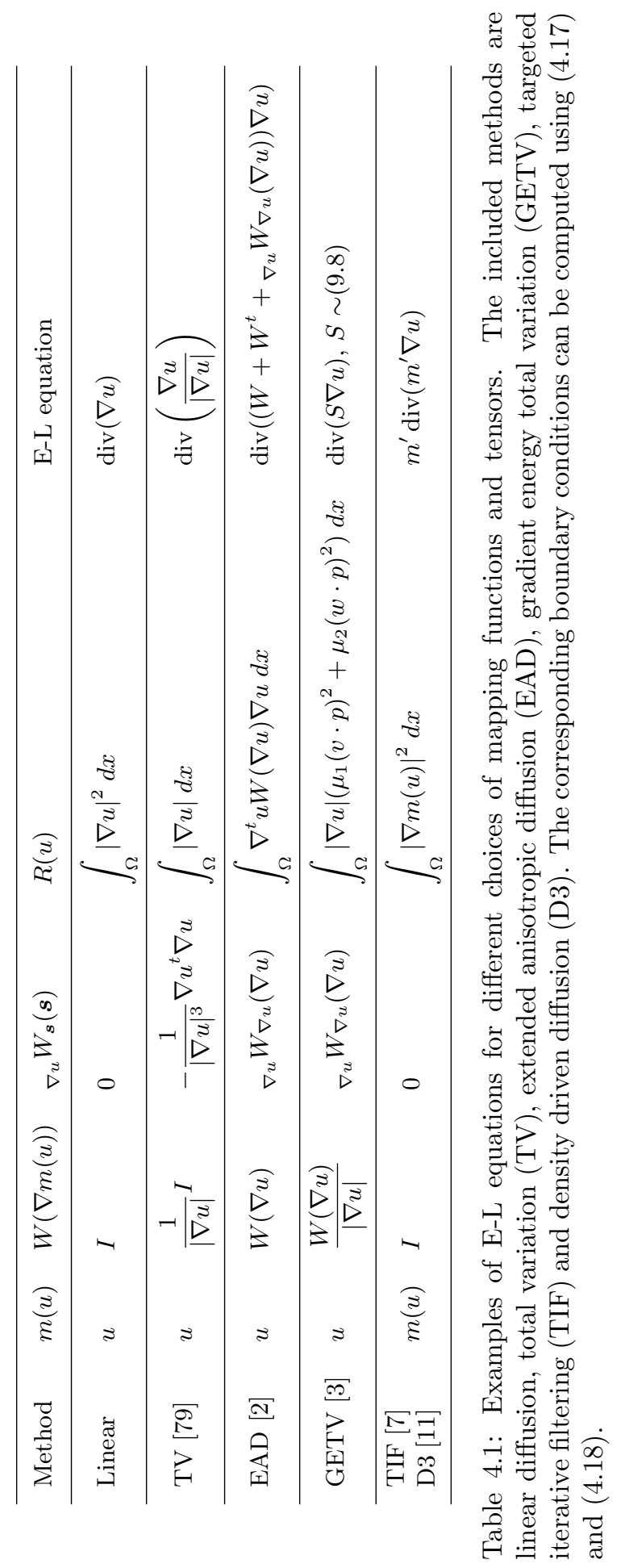


Corollary 1 (Constraints symmetric tensor). Let $W \in \mathbb{R}^{2 \times 2}$ be a symmetric tensor. If the conditions
i) $b_{s_{1}}=a_{s_{2}}$,
ii) $c_{s_{1}}=b_{s_{2}}$,

are both satisfied, then ${ }_{\nabla u} W_{\boldsymbol{s}}(\boldsymbol{s})$ is also a symmetric tensor.

Proof. Since $W$ is a symmetric tensor and is given by 4.20$)$, then ${ }_{\nabla u} W_{\boldsymbol{s}}(\boldsymbol{s})$ can be written as

$$
{ }_{\nabla u} W_{\boldsymbol{s}}(\boldsymbol{s})=u_{x}\left(\begin{array}{ll}
a_{s_{1}} & b_{s_{1}} \\
a_{s_{2}} & b_{s_{2}}
\end{array}\right)+u_{y}\left(\begin{array}{ll}
b_{s_{1}} & c_{s_{1}} \\
b_{s_{2}} & c_{s_{2}}
\end{array}\right) .
$$

Conditions $i$ ) and $i i)$ follow from the constraint ${ }_{\nabla u} W_{\boldsymbol{s}}(\boldsymbol{s})=\left({ }_{\nabla u} W_{\boldsymbol{s}}(\boldsymbol{s})\right)^{t}$.

The solution of $i$ ) and $i i$ ) is not unique. For example, one solution is given by the constraint

$$
b=\int a_{s_{2}} d s_{1}=\int c_{s_{1}} d s_{2} .
$$

If instead $b$ is given then $a$ and $c$ must satisfy

$$
\begin{aligned}
& a=\int b_{s_{1}} d s_{2}, \\
& c=\int b_{s_{2}} d s_{1},
\end{aligned}
$$

which result in

$$
W=\left(\begin{array}{cc}
\int b_{s_{1}} d s_{2} & b \\
b & \int b_{s_{2}} d s_{1}
\end{array}\right) .
$$

Note that in the general case it may be difficult, or not interesting, to evaluate Corollary 1. However, as a theoretical tool it may serve the purpose to derive classes of tensors $S$ that are symmetric.

\section{Contraction to single divergence}

The below lemma reformulates the E-L equation 4.16 on a single divergence form under certain conditions.

Lemma 1 (Contraction of divergence forms). Given a tensor $C \in \mathbb{R}^{n \times n}$ the following relation holds

$$
\left(m^{\prime}\right)^{2} \operatorname{div}(C \nabla u)+\operatorname{div}\left(\left(m^{\prime}\right)^{2} C \nabla u\right)=2 m^{\prime} \operatorname{div}\left(m^{\prime} C \nabla u\right) .
$$


Proof. Using the identity $\operatorname{div}(C \nabla u)=\operatorname{div}(C) \nabla u+\operatorname{tr}(C H u)$, where $H$ is the Hessian matrix, the left hand-side of 4.26 can be written as

$$
\begin{aligned}
& \left(m^{\prime}\right)^{2}(\operatorname{div}(C) \nabla u+\operatorname{tr}(C H u)) \\
& \quad+\operatorname{div}\left(\left(m^{\prime}\right)^{2} C\right) \nabla u+\operatorname{tr}\left(\left(m^{\prime}\right)^{2} C H u\right) \\
& =\left(\left(m^{\prime}\right)^{2} \operatorname{div}(C)+2 m^{\prime} m^{\prime \prime} \nabla^{t} u C+\left(m^{\prime}\right)^{2} \operatorname{div}(C)\right) \nabla u \\
& \quad+2\left(m^{\prime}\right)^{2} \operatorname{tr}(P H u) \\
& =2 m^{\prime}\left[\operatorname{div}\left(m^{\prime} C\right) \nabla u+m^{\prime} \operatorname{tr}(C H u)\right],
\end{aligned}
$$

which concludes the proof.

For an extended proof see appendix A.2.

Remark 1 (Cancellation of negative sign). Observe that depending on $m$, its derivative $m^{\prime}$ can in the general case take negative values. However, due to linearity of the divergence operator any non-negative factor originating from $m^{\prime}$ cancels out as indicated by the identity in Lemma 1, i.e.,

$$
\left( \pm m^{\prime}\right) \operatorname{div}\left(\left( \pm m^{\prime}\right) C \nabla u\right) \Longleftrightarrow+m^{\prime} \operatorname{div}\left(m^{\prime} C \nabla u\right) .
$$

Assuming that $C$ is at least positive (semi)-definite, then the E-L equations will have a convergent behaviour.

With the sufficient condition for tensor symmetry of $W$ and ${ }_{\nabla u} W_{\boldsymbol{s}}(\boldsymbol{s})$, and the above simplification of the divergence terms in Lemma 1, we obtain the E$\mathrm{L}$ equation of the regularization term stated in Theorem 2 on a (considerably) simplified form.

Corollary 2 (Contraction with symmetry constraint). Let $W \in \mathbb{R}^{n \times n}$ be a symmetric tensor, then the E-L equation (4.16) is given by

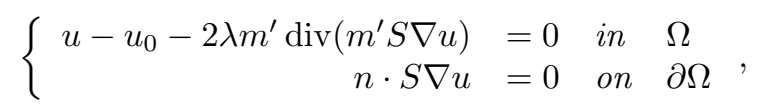

where

$$
S=2 W+m_{\nabla u}^{\prime} W_{\boldsymbol{s}}(\boldsymbol{s}) .
$$

Proof. Since $W=W^{t}$ it follows that $S_{1}=S_{2}$. Then by using Lemma 1 equation 4.31 follows.

\section{Convexity}

This part discuss and present results on the convexity of the functional in the case $m(u)=u$. If $m(u)$ is a monotonically increasing function, the below results can be generalised using similar arguments. For a general function $m(u)$ the description of a convex function remains an open problem. 
The approach is based on the eigenvalue decomposition of the involved tensors and thus relies on the existence of an eigenvalue decomposition of $W(\nabla u)$ with real eigenvalues $\lambda_{1}, \lambda_{2}$ and eigenvectors $v, w$. The formulation is not as unintuitive as it may seem at first. Recall that a tensor always has a well defined eigendecomposition, i.e., the eigenvectors are not singular. Start by rewriting the quadratic form $\nabla^{t} u W(\nabla u) \nabla u$ obtained from 4.15 with $m(u)=u$ in the following way:

$$
\begin{aligned}
\nabla^{t} u W(\nabla u) \nabla u & =\nabla^{t} u\left[\lambda_{1} v v^{t}+\lambda_{2} w w^{t}\right] \nabla u \\
& =\lambda_{1} v^{t}\left[\nabla u \nabla^{t} u\right] v+\lambda_{2} w^{t}\left[\nabla u \nabla^{t} u\right] w .
\end{aligned}
$$

The product $\nabla u \nabla^{t} u$ is a rank-1 tensor with orthonormal eigenvectors $p=\left(p_{1}, p_{2}\right)^{t}$ and $p^{\perp}=\left(p_{2},-p_{1}\right)^{t}$ such that $P=\left(p, p^{\perp}\right)$ and $\Lambda$ has the corresponding eigenvalues $\kappa_{1}$ and $\kappa_{2}$ on its diagonal. Note that, by the spectral theorem, the eigendecomposition of $\nabla u \nabla^{t} u$ is well-defined, i.e., the eigenvector $p$ is not singular. This is shown by the generalised definition of $p$, i.e., in the case of $|\nabla u| \neq 0$ then $p=\nabla u /|\nabla u|$, and if $|\nabla u|=0$ let $P=I$ where $I$ is the identity matrix.

Now substitute the eigendecomposition of $\nabla u \nabla^{t} u=P^{t} \Lambda P$ into 4.33:

$$
\begin{aligned}
\nabla^{t} u W(\nabla u) \nabla u & =\left(\lambda_{1} v^{t} P \Lambda P^{t} v+\lambda_{2} w^{t} P \Lambda P^{t} w\right) \\
& =\left(\lambda_{1} v^{t} P\left(\begin{array}{cc}
\kappa_{1} & 0 \\
0 & \kappa_{2}
\end{array}\right) P^{t} v+\lambda_{2} w^{t} P\left(\begin{array}{cc}
\kappa_{1} & 0 \\
0 & \kappa_{2}
\end{array}\right) P^{t} w\right),
\end{aligned}
$$

and insert $\kappa_{1}=|\nabla u|^{2}$ and $\kappa_{2}=0$. Rewriting (4.34) we obtain

$$
\nabla^{t} u W(\nabla u) \nabla u=\lambda_{1}|\nabla u|^{2}(v \cdot p)^{2}+\lambda_{2}|\nabla u|^{2}(w \cdot p)^{2} .
$$

The interpretation of 4.35 is that $v, p, w$ are normalized eigenvectors such that the two scalar products define the rotation of $W(\nabla u)$ in relation to the image gradients direction. This can be illustrated by using the definition of the scalar product, i.e., $v \cdot p=\cos (\theta)$ and $w \cdot p=\sin (\theta)$, where $\theta$ is the rotation angle as shown in figure 4.1 (a). Note that if $W(\nabla u)$ describes the local directional information, its eigenvectors will be parallel to the orthonormal eigenvectors of $\nabla u \nabla^{t} u$, i.e., $v \| p$ and $w \| p^{\perp}$ if $\theta=0$. Now consider the convexity of the proposed functional.

Corollary 3 (Convex functional). The functional $R(u)$ is convex w.r.t. the element $u$.

Proof. To prove the convexity of $R(u)$, write $\Phi(u)=\nabla^{t} u W(\nabla u) \nabla u$ in terms of the eigenvectors and eigenvalues of $W(\nabla u)$. Then, from 4.35 it follows that

$$
\begin{aligned}
\Phi(\nabla u) & =|\nabla u|^{2}\left(\lambda_{1} p^{t}\left(v v^{t}\right) p+\lambda_{2} p^{t}\left(w w^{t}\right) p\right) \\
& =|\nabla u|^{2} p^{t}\left(\lambda_{1} v v^{t}+\lambda_{2} w w^{t}\right) p \\
& =(V p)^{t}\left(\begin{array}{cc}
\tau_{1} & 0 \\
0 & \tau_{2}
\end{array}\right) V p,
\end{aligned}
$$

where $V=(v, w)$ and $\tau_{i}(\nabla u)=|\nabla u|^{2} \lambda_{i} \geq 0$ for $i=1,2$. Let $q=V p=\left(q_{1}, q_{2}\right)^{t}$, then

$$
\Phi(\nabla u)=\tau_{1} q_{1}^{2}+\tau_{2} q_{2}^{2},
$$




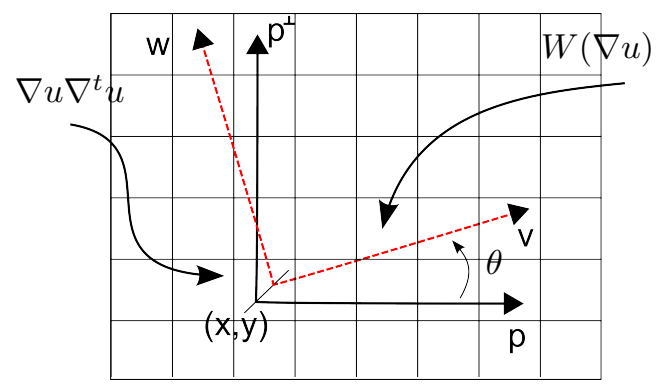

(a)

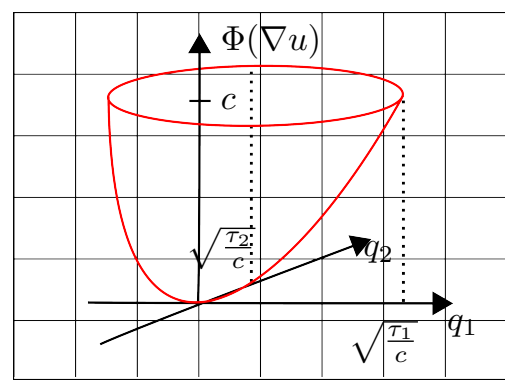

(b)

Figure 4.1: (a) Illustration of eigenvector basis at coordinate $(x, y)$, the dashed (red) arrows indicate the eigenvectors of $W(\nabla u)$ and $S(\nabla u)$ and thick (black) arrows $\nabla u \nabla^{t} u$. (b) Illustration of the paraboloid (4.37) where we set $c=\tau_{1} q_{1}^{2}+$ $\tau_{2} q_{2}^{2}$.

is a quadratic form in the basis of orthonormal eigenvectors $V$ and $\tau_{i}(\nabla u)$. This quadratic form is always well-defined due to the spectral theorem. Since the paraboloid 4.37 has positive curvature everywhere, and $u$ maps continuously to the paraboloid, $R(u)$ is convex in $u$ which concludes the proof.

The paraboloid $\Phi(\nabla u)$ is illustrated in figure 4.1 (b).

In 4.37 we defined $q=V p$ such that the paraboloid is represented in the $q_{1}$ - $q_{2}$ plane. Thus it is of interest to further investigate the structure of this plane. It turns out that the $q$ vector is always a vector of unit length. To show this statement, consider the following relations

$$
|q|^{2}=q^{t} q=(V p)^{t} V p=p^{t}\left(\begin{array}{cc}
v_{1}^{2}+v_{2}^{2} & v_{1} w_{1}+v_{2} w_{2} \\
v_{1} w_{1}+v_{2} w_{2} & w_{1}^{2}+w_{2}^{2}
\end{array}\right) p .
$$

Since $v$ and $w$ are two orthonormal eigenvectors, $v \cdot w=v_{1} w_{1}+v_{2} w_{2}=0$ and $|v|=|w|=1$. Then, since $|p|=1$ is an eigenvector, equation (4.38) can be rewritten to yield the final result

$$
|q|^{2}=p^{t}\left(\begin{array}{ll}
1 & 0 \\
0 & 1
\end{array}\right) p=p^{t} p=1
$$

Remark 2 (Relation Schatten-norm). The expression (4.35) can be expressed in the Schatten-1 norm [49] (pp. 441), i.e., $\|A\|_{1}=\sum_{i}^{n}\left|\sigma_{i}(A)\right|$ where $\sigma_{i}(A)$ denotes the $i$ 'th singular value of the tensor $A$. Since $\|A\|_{1}$ is a norm, it has the important properties of positivity and convexity. However, in our case it is not obvious that the convexity follow directly from the norm due to the (possibly) non-linearity of $W(\nabla u)$, see Corollary 3. From (4.35) we have that

$$
\nabla^{t} u W(\nabla u) \nabla u=\|A(\nabla u)\|_{1} .
$$

This means that $A(\nabla u)$ has singular values $\sigma_{1}(A)=\lambda_{1}|\nabla u|^{2}(v \cdot p)^{2}$ and $\sigma_{2}(A)=$ $\lambda_{2}|\nabla u|^{2}(w \cdot p)^{2}$ where $\lambda_{1,2}$ are the eigenvalues of $W(\nabla u)$. 


\section{Extended anisotropic diffusion}

Extended anisotropic diffusion (EAD) [2] is obtained from the Euler-Lagrange equation of Theorem 2. Let $W$ be the structure tensor such that $W=W^{t}$ and define

$$
C(\nabla u)=\frac{1}{2}\left(S_{1}+S_{2}\right)=2 W+{ }_{\nabla u} W_{\nabla u}(\nabla u) .
$$

Then, by using the linearity of the divergence operator we make the following definition.

Definition 1 (Extended anisotropic diffusion). The extended anisotropic diffusion (EAD) scheme is

$$
\partial_{t} u-\lambda\left(2 \operatorname{div}\left(D_{1}(W) \nabla u\right)+\operatorname{div}\left(D_{2}\left({ }_{\nabla u} W_{\nabla u}(\nabla u)\right) \nabla u\right)\right)=0 .
$$

In the above definition $D_{1}$ and $D_{2}$ denote the transformation of the respective tensors eigenvalues of $W$ and ${ }_{\nabla u} W_{\nabla u}(\nabla u)$. Since ${ }_{\nabla u} W_{\nabla u}(\nabla u)$ is (in general) not a symmetric tensor its eigenvalues may be complex-valued. However, in practice, the imaginary part is small and can thus be neglected. See section 13.1 for additional details on the exact choice of diffusivity function.

\subsection{Study of the inverse problem}

The focus of the previous sections was on deriving an Euler-Lagrange equation from a variational formulation. This section considers the classical inverse problem, i.e.

- What are the necessary conditions such that a variational formulation can be obtained from a given tensor-based PDE?

The explicit relation between the anisotropic PDE and a variational formulation remains unsolved. For example, Black and Rangarajan [17] attempted to describe the connection for scalar-valued non-linear diffusion methods using the framework of robust statistics.

In this work we consider the problem from an algebraic point of view. This leads us to necessary conditions (NC) for the existence of an energy functional of the type in 4.15 given a tensor-based diffusion PDE 4.16. Note that our approach is restricted to $m(u)=u$. The theorem below is an adaptation from our work [2].

Theorem 3 (NC for PDE to functional). For a given tensor $\tilde{S}=\tilde{S}\left(u_{x}, u_{y}\right)$ with entities $s^{(i)}$ satisfying the necessary condition

$$
s^{(3)}+u_{x} s_{u_{x}}^{(3)}-u_{x} s_{u_{y}}^{(1)}=s^{(2)}+u_{y} s_{u_{y}}^{(2)}-u_{y} s_{u_{x}}^{(4)},
$$

there exists a symmetric tensor $W\left(u_{x}, u_{y}\right)$ such that the tensor-based diffusion $P D E$

$$
\left\{\begin{array}{rlrl}
u-u_{0}-\operatorname{div}(\tilde{S} \nabla u) & =0 & & \text { in } \Omega \\
\tilde{S} \nabla u \cdot n=0 & & \text { on } \partial \Omega
\end{array},\right.
$$


is the E-L equation to the functional $E(u)$ in 4.15), with $m(u)=u$. Moreover, the entities in

$$
W\left(u_{x}, u_{y}\right)=\left(\begin{array}{ll}
f\left(u_{x}, u_{y}\right) & g\left(u_{x}, u_{y}\right) \\
g\left(u_{x}, u_{y}\right) & h\left(u_{x}, u_{y}\right)
\end{array}\right),
$$

are given below. First we consider $g\left(u_{x}, u_{y}\right)=\tilde{g}(\alpha, \beta)$, where

$$
\tilde{g}(\alpha, \beta)=\frac{1}{\alpha^{2}} \int \alpha \tilde{R}(\alpha, \beta) d \alpha+\frac{1}{\alpha^{2}} \tilde{\theta}(\beta),
$$

and $\alpha=u_{x}, \beta=u_{x} / u_{y}$ and $\tilde{R}(\alpha, \beta)=R\left(u_{x}, u_{y}\right)$ is the right-hand side of $N C$ 4.43.). Second, $f$ and $h$ are obtained from

$$
\begin{aligned}
& f\left(u_{x}, u_{y}\right)=\frac{1}{u_{x}^{2}} \int_{0}^{u_{x}}\left(\xi s^{(1)}\left(\xi, u_{y}\right)-\xi u_{y} g_{\xi}\left(\xi, u_{y}\right)\right) d \xi+\frac{1}{u_{x}^{2}} \rho\left(u_{y}\right), \\
& h\left(u_{x}, u_{y}\right)=\frac{1}{u_{y}^{2}} \int_{0}^{u_{y}}\left(\eta s^{(4)}\left(u_{x}, \eta\right)-u_{x} \eta g_{\eta}\left(u_{x}, \eta\right)\right) d \eta+\frac{1}{u_{y}^{2}} \zeta\left(u_{x}\right),
\end{aligned}
$$

where $\rho, \zeta$ and $\tilde{\theta}$ are arbitrary functions.

Proof. Let the system of equations,

$$
\left\{\begin{array}{l}
u_{x} f_{u_{x}}+u_{y} g_{u_{x}}+2 f=s^{(1)} \\
u_{x} g_{u_{x}}+u_{y} h_{u_{x}}+2 g=s^{(2)} \\
u_{x} f_{u_{y}}+u_{y} g_{u_{y}}+2 g=s^{(3)} \\
u_{x} g_{u_{y}}+u_{y} h_{u_{y}}+2 h=s^{(4)}
\end{array}\right.
$$

represent the expansion of 9.8 with given notation. The function $f$ in 4.47 is obtained from 4.49 by solving

$$
\frac{\partial}{\partial u_{x}}\left(u_{x}^{2} f\right)=u_{x} s^{(1)}-u_{x} u_{y} g_{u_{x}} .
$$

To construct $g$, differentiate $f$ in 4.47 with respect to $u_{y}$. This gives that

$$
f_{u_{y}}\left(u_{x}, u_{y}\right)=\frac{1}{u_{x}^{2}} \int_{0}^{u_{x}}\left(\xi s_{u_{y}}^{(1)}\left(\xi, u_{y}\right)-\xi g_{\xi}\left(\xi, u_{y}\right)-\xi u_{y} g_{\xi u_{y}}\left(\xi, u_{y}\right)\right) d \xi+\frac{1}{u_{x}^{2}} \rho^{\prime}\left(u_{y}\right) .
$$

Now, insert $f_{u_{y}}$ into 4.51 and differentiate with respect to $u_{x}$ to obtain

$$
u_{x} g_{u_{x}}+u_{y} g_{u_{y}}+2 g=s^{(3)}+u_{x} s_{u_{x}}^{(3)}-u_{x} s_{u_{y}}^{(1)} .
$$

In the same way, solve for $h$ in 4.50 to obtain 4.48). Substituting 4.50 into 4.52 yields

$$
u_{x} g_{u_{x}}+u_{y} g_{u_{y}}+2 g=s^{(2)}+u_{y} s_{u_{y}}^{(2)}-u_{y} s_{u_{x}}^{(4)} .
$$

Comparing 4.55) and 4.56), we deduce the necessary condition to establish the existence of $g, f$ and $h$. From NC we are now able to compute $g$ satisfying

$$
u_{x} g_{u_{x}}+u_{y} g_{u_{y}}+2 g=R\left(u_{x}, u_{y}\right),
$$


where $R$ is the right-hand side (or the left-hand side) in NC. Changing variables $\alpha=u_{x}$ and $\beta=u_{x} / u_{y}$, in 4.57 we get

$$
\tilde{g}_{\alpha}\left(u_{x} \alpha_{u_{x}}+u_{y} \alpha_{u_{y}}\right)+\tilde{g}_{\beta}\left(u_{x} \beta_{u_{x}}+u_{y} \beta_{u_{y}}\right)+2 \tilde{g}=\tilde{R}(\alpha, \beta),
$$

where $\tilde{R}(\alpha, \beta)=R\left(u_{x}, u_{y}\right)$ and $\tilde{g}(\alpha, \beta)=g\left(u_{x}, u_{y}\right)$. Since $\alpha_{u_{x}}=1, \alpha_{u_{y}}=0$ and $\beta_{u_{x}}=1 / u_{y} \beta_{u_{y}}=-u_{x} / u_{y}^{2}$, equation 4.58 can then be writen as $\alpha \tilde{g}_{\alpha}+2 \tilde{g}=\tilde{R}$. We solve for $g$ in the following way

$$
\frac{\partial}{\partial \alpha}\left(\alpha^{2} \tilde{g}\right)=\alpha \tilde{R}(\alpha, \beta) \Leftrightarrow \tilde{g}=\frac{1}{\alpha^{2}} \int \alpha \tilde{R}(\alpha, \beta) d \alpha+\frac{1}{\alpha^{2}} \tilde{\theta}(\beta),
$$

where $\tilde{\theta}$ is an arbitrary function. Put $\tilde{\theta}(\alpha, \beta)=\theta\left(u_{x}, u_{y}\right)$ to obtain $g$ in 4.46 . Now $f$ and $h$ can be obtained from (4.47) and 4.48).

Corollary 4 (NC conditions for PDE to functional). For the tensor $\tilde{S}=\nabla u \nabla u^{t}$ there exists a tensor

$$
W=\frac{1}{4}\left(\begin{array}{cc}
u_{x}^{2} & u_{x} u_{y} \\
u_{x} u_{y} & u_{y}^{2}
\end{array}\right),
$$

such that the following PDE

$$
\left\{\begin{array}{rlrl}
u-u_{0}-\operatorname{div}(\tilde{S} \nabla u) & =0 & & \text { in } \Omega \\
\tilde{S} \nabla u \cdot n=0 & & \text { on } \partial \Omega
\end{array}\right.
$$

is the E-L equation of the functional $E(u)$ in 4.15$)$, for $m(u)=u$.

Proof. It is straighforward to verify that the entities of the tensor $\tilde{S}$ satisfies the $\mathrm{NC}$ condition where the right-hand side 4.43

$$
R\left(u_{x}, u_{y}\right)=2 u_{x} u_{y}
$$

For simplicity choose $\theta, \rho$, and $\zeta$ to be zero in 4.46 - 4.48). With change of variables $\alpha=u_{x}$ and $\beta=u_{x} / u_{y}, 4.62$ become $R(\alpha, \beta)=2 \alpha^{2} / \beta$ so that 4.46 is $\tilde{g}(\alpha, \beta)=\frac{\alpha^{2}}{2 \beta}$, then

$$
g\left(u_{x}, u_{y}\right)=\frac{1}{2} u_{x} u_{y}
$$

Using (4.47) and (4.48), $f$ and $h$ are

$$
\begin{aligned}
& f\left(u_{x}, u_{y}\right)=\frac{1}{u_{x}^{2}} \int_{0}^{u_{x}}\left(\xi^{3}-\xi u_{y}^{2}\right) d \xi=\frac{u_{x}^{2}}{4}-\frac{u_{y}^{2}}{4}, \\
& h\left(u_{x}, u_{y}\right)=\frac{1}{u_{y}^{2}} \int_{0}^{u_{y}}\left(\eta^{3}-\eta u_{x}^{2}\right) d \eta=\frac{u_{y}^{2}}{4}-\frac{u_{x}^{2}}{4} .
\end{aligned}
$$

Let $\widehat{W}$ be the tensor defined by 4.63 - 4.65, then put this tensor in 4.15 with $m(u)=u$ and obtain

$$
\begin{aligned}
\nabla u^{t} \widehat{W} \nabla u & =\nabla u^{t}\left(\begin{array}{cc}
u_{x}^{2}-u_{y}^{2} & 2 u_{x} u_{y} \\
2 u_{x} u_{y} & u_{y}^{2}-u_{x}^{2}
\end{array}\right) \nabla u \\
& =\nabla u^{t}\left(\begin{array}{cc}
u_{x}^{2} & u_{x} u_{y} \\
u_{x} u_{y} & u_{y}^{2}
\end{array}\right) \nabla u=\nabla u^{t} W \nabla u
\end{aligned}
$$


neglecting the factor $1 / 4$. This concludes the proof and 4.60 follows from 4.66.

\subsubsection{Weighted tensor-based variational formulation}

This part considers the case when the tensor in the PDE is defined with a postconvolution of the tensor-components. Thus, let $W$ be a weighted tensor

$$
W=\left(w *\left(\begin{array}{ll}
f\left(u_{x}, u_{y}\right) & g\left(u_{x}, u_{y}\right) \\
g\left(u_{x}, u_{y}\right) & h\left(u_{x}, u_{y}\right)
\end{array}\right)\right)(x, y),
$$

where $*$ is the convolution operator and $w$ is a smooth kernel. Now, the analogous problem to the previous section is to formulate conditions such that the functional

$$
E(u)=\frac{1}{2} \int_{\Omega}\left(u-u_{0}\right)^{2} d x+\lambda \int_{\Omega} \nabla u^{t} W(\nabla u) \nabla u d x
$$

has

$$
\left\{\begin{array}{rl}
u-u_{0}-\lambda \operatorname{div}(S \nabla u)=0 & \text { in } \Omega \\
S \nabla u \cdot n=0 & \text { on } \partial \Omega
\end{array},\right.
$$

as the corresponding E-L equation where $S=w *\left(\nabla u \nabla u^{t}\right)$.

Proposition 1. For the tensor $S=w *\left(\nabla u \nabla u^{t}\right)$, the PDE (4.69) is the E-L equation of the functional (4.68) with $W$ as in (4.67) if

$$
u_{x}(x, y)\left(w * g_{u_{x}}\right)(x, y)=u_{y}(x, y)\left(w * g_{u_{y}}\right)(x, y),
$$

is satisfied. Moreover, the entities of $W$ can be obtained by solving the following system of differential equations

$$
\left\{\begin{aligned}
u_{x}\left(w * f_{u_{x}}\right)+u_{y}\left(w * g_{u_{x}}\right)+2(w * f) & =w * u_{x}^{2} \\
u_{x}\left(w * g_{u_{x}}\right)+2(w * g) & =w *\left(u_{x} u_{y}\right) \\
u_{y}\left(w * g_{u_{y}}\right)+2(w * g) & =w *\left(u_{x} u_{y}\right) \\
u_{x}\left(w * g_{u_{y}}\right)+u_{y}\left(w * h_{u_{y}}\right)+2(w * h) & =w * u_{y}^{2}
\end{aligned}\right.
$$

We have chosen to include $w$ in the formulation of the tensor (4.67). This will allow us to include $u_{x}$ and $u_{y}$ inside the convolution operation in (4.70) assuming that $u_{x}$ and $u_{y}$ are sampled at different positions on the grid. Also, Theorem 3 describes the case when $\operatorname{rank}(S)=1$, i.e., the signal has an intrinsically onedimensional structure (such as lines). Proposition 1 describes the general case when $\operatorname{rank}(S)=2$. To summarise, the above results is a framework which states under what conditions a given PDE is the corresponding E-L equation to a tensorbased image diffusion energy functional. 


\section{Chapter 5}

\section{Scalar-valued diffusion in non-linear domains}

Theorem 2 in the previous chapter describes a general framework to obtain tensorbased diffusion formulations. This chapter considers scalar-valued formulations, and explore properties of the resulting schemes. The derived results can be interpreted as generalisations of both the isotropic diffusion and the Perona and Malik diffusion discussed in previous chapters.

\section{$5.1 p$-Norm formulation}

This section studies the connection between Theorem 2 and the following $p$-norm

$$
|\nabla u|^{p}=\left(\sqrt{\nabla^{t} u \nabla u}\right)^{p}
$$

where $1 \leq p<\infty$. If $(5.1)$ is used as the integrand in a regularization term, then, for $p=1$ the resulting regularization terms reduce to standard total variation 79 . and if $p=2$ it describes the case of isotropic diffusion. The below proposition relates the quadratic form in Theorem 2 to the functional

$$
E(u)=\int_{\Omega}\left(u-u_{0}\right)^{2} d x+\frac{\lambda}{p} \int_{\Omega}|\nabla m(u)|^{p} d x,
$$

where $\lambda$ is the smoothness parameter.

Proposition 2 (Scalar-valued diffusion in non-linear domains). Let I be the identity matrix and set $W(\nabla m(u))=|\nabla m(u)|^{q} I$ in Theorem 2. Let $q=p-2$ where $1 \leq p<\infty$, then

$$
\int_{\Omega}|\nabla m(u)|^{p} d x=\int_{\Omega} \nabla^{t} m(u)|\nabla m(u)|^{q} I \nabla m(u) d x .
$$


Proof. Substituting $W(\nabla m(u))=|\nabla m(u)|^{q} I$ into the quadratic form and rearrange the resulting terms yields

$$
\begin{aligned}
\int_{\Omega} \nabla^{t} m(u) W(\nabla m(u)) \nabla m(u) d x & =\int_{\Omega} \nabla^{t} m(u)|\nabla m(u)|^{q} I \nabla m(u) d x \\
& =\int_{\Omega} \nabla^{t} m(u)|\nabla m(u)|^{p-2} I \nabla m(u) d x \\
& =\int_{\Omega}|\nabla m(u)|^{p} d x,
\end{aligned}
$$

which concludes the proof.

The next section introduces the 2-norm formulation expressed in terms of the mapping function. The resulting scheme is denoted as targeted iterative filtering [7. These results are followed by another formulation of particular interest: the 1-norm formulation corresponding to total variation with a non-linear mapping function.

\subsection{Isotropic diffusion in non-linear domains}

This section considers the isotropic case of $p=2$ in Proposition 2, i.e., the mapping function is introduced into the framework of isotropic diffusion 3.3 . The material is an adaptation of our work [7] and shows the derivation of the necessary condition for a minimum, the E-L equation. Additionally, a detailed investigation on sufficient conditions to obtain a local minimum is presented.

The aim of exploiting the mapping function is to simultaneously consider the signal domain and the domain-dependent transformation of the image data. To achieve this behaviour, it is natural to express the regularization term of the energy functional 3.2 in the transformed domain.

The domain-application considered in this section is medical visualization. In this context, the mapping function is defined by a non-linear "transfer function" which maps the value-range of medical imaging data to better visualize structure details in the images. Additionally, it is shown that the defined mapping function satisfies the sufficient conditions to obtain (at least) a local minimum. Section 12.1 below presents the experimental evaluation and shows that the selection of transfer function satisfies the necessary and sufficient conditions for a local minimum.

\subsubsection{Necessary conditions for local minimum}

Let $m(u)$ be the mapping function that maps $u$ to its application domain, then for $p=2$ in Proposition 2 define the functional

$$
E(u)=\int_{\Omega}\left(u-u_{0}\right)^{2} d x+\lambda \int_{\Omega}|\nabla m(u)|^{2} d x,
$$

where $\lambda>0$ is a parameter determining the influence of the regularization term. 
The variational derivative of the the regularization term of $E(u)$ in 5.5 is computed using the Gâteaux derivative in section 2.2.7 and we get

$$
\partial_{u} R(u) v=\lim _{\varepsilon \rightarrow 0} \int_{\Omega} \frac{|\nabla m(u+\varepsilon v)|^{2}-|\nabla m(u)|^{2}}{\varepsilon} d x .
$$

Using the chain rule $\nabla m(u)=m^{\prime}(u) \nabla u, 5.6$ is reformulated as

$$
\begin{aligned}
\partial_{u} R(u) v=\lim _{\varepsilon \rightarrow 0} \frac{1}{\varepsilon} \int_{\Omega}|\nabla u|^{2}\left(m^{\prime}(u+\varepsilon v)^{2}-m^{\prime}(u)^{2}\right) \\
+m^{\prime}(u+\varepsilon v)^{2}\left(\varepsilon 2 \nabla u^{t} \nabla v+\varepsilon^{2}|\nabla v|^{2}\right) d x,
\end{aligned}
$$

and by Green's identity and Neumann boundary conditions

$$
\partial_{u} R(u) v=\int_{\Omega}\left(2|\nabla u|^{2} m^{\prime}(u) m^{\prime \prime}(u)-2 \operatorname{div}\left(m^{\prime}(u)^{2} \nabla u\right)\right) v d x .
$$

Now observe that

$$
\operatorname{div}\left(m^{\prime}(u)^{2} \nabla u\right)=2 m^{\prime}(u) m^{\prime \prime}(u)|\nabla u|^{2}+m^{\prime}(u)^{2} \Delta u,
$$

and by using this result in $(5.8)$, and since $v \neq 0$, the E-L equation corresponding to 5.5 reads

$$
\left\{\begin{array}{rll}
u-u_{0}-\lambda\left(\operatorname{div}\left(m^{\prime}(u)^{2} \nabla u\right)+m^{\prime}(u)^{2} \Delta u\right) & =0 & \text { in } \quad \Omega \\
\nabla u \cdot n & =0 & \text { on } \partial \Omega
\end{array} .\right.
$$

Compared to (3.13), the divergence operator is modulated with the squared steepness of the mapping function. Also, the Laplacian is weighted with the same factor. If and only if $m$ is a globally linear function, 5.10 becomes identical to (3.9). The difference when compared to non-linear diffusion is easiest explained in terms of the Lagrangian: Replacing $\Psi(|\nabla u|)$ with $m^{\prime}(u)^{2}$ means replacing the robust error function with an intensity dependent factor.

\subsubsection{Sufficient conditions for local minimum}

This section derives sufficient conditions for the solution of the E-L equation to be a minimum of the regularization term in (5.5). The result is summarised in the theorem below. Note that if the mapping function is a strict monotone function, the regularization term in $(5.5)$ is obviously convex and the necessary condition is also a sufficient condition (SC). However, in the general case, $m$ is not always a strict monotone function, and this is the case we consider here.

Theorem 4 (SC local minimum for isotropic diffusion in non-linear domains). Let $u_{0}$ be an observed image in a domain $\Omega \subset \mathbb{R}^{2}$, and denote by $E(u)$ the functional

$$
E(u)=\int_{\Omega}\left(u-u_{0}\right)^{2} d x+\lambda \int_{\Omega}|\nabla m(u)|^{2} d x,
$$


where $u \in \mathcal{C}^{2}$ and $m(u) \in \mathcal{C}^{3}$. Let $\varepsilon>0$ be arbitrary and consider the set

$$
B_{\varepsilon}=\left\{h, \nabla h \in L^{2}(\Omega):\|h\|_{L^{2}(\Omega)}^{2} \leq \varepsilon^{2} / C_{M}, \quad\|\nabla h\|_{L^{2}(\Omega)}^{2} \geq \varepsilon\right\},
$$

where

$$
C_{M}=\left.\max _{\boldsymbol{x} \in \Omega}\left|\left[m^{\prime}\left(u^{*}(x)\right) m^{\prime \prime \prime}\left(u^{*}(x)\right)-3\left(m^{\prime \prime}\left(u^{*}(x)\right)\right)^{2}\right]\right| \nabla u^{*}(x)\right|^{2} \mid .
$$

Then $u^{*}$ is a local minimum of $E(u)$ given by the solution of the E-L equation (5.10) if there exists $\xi \in \Omega$ such that

$$
\left(m^{\prime}\left(u^{*}(\xi)\right)\right)^{2}\|\nabla h\|_{L^{2}(\Omega)}^{2}>\varepsilon^{2}
$$

for every $h \in B_{\varepsilon}$.

Proof. In order to find the sufficient condition for a minimum, define the regularization term in the functional as

$$
R(u)=\int_{\Omega}|\nabla m(u)|^{2} d x=\int_{\Omega}\left(m^{\prime}(u)\right)^{2}|\nabla u|^{2} d x .
$$

Given a function $\varphi \in \mathcal{C}^{3}$, a third order Taylor expansion at the point 0 is

$$
\varphi(a)-\varphi(0)=a \varphi^{\prime}(0)+\frac{a^{2}}{2} \varphi^{\prime \prime}(0)+\frac{a^{3}}{6} \varphi^{\prime \prime \prime}(a \theta), \quad 0<\theta<1 .
$$

Let $h \in \mathcal{C}^{1}$, then define $\varphi(a)=R(u+a h)$, which determines the first variation $\delta R$ of $R(u)$ as

$$
\delta R=\lim _{a \rightarrow 0} \frac{R(u+a h)-R(u)}{a}=\lim _{a \rightarrow 0} \frac{\varphi(a)-\varphi(0)}{a}=\varphi^{\prime}(0) .
$$

In the same way the second variation $\delta^{2} R$ follows. Since $\delta R$ is a linear functional in $h$ and $\delta^{2} R$ is a quadratic form in $h$, define $L_{1}(h)=\delta R=\varphi^{\prime}(0)$ and $L_{2}(h, h)=$ $\delta^{2} R=\varphi^{\prime \prime}(0)$. Given that $\varphi$ is differentiable then so is $R$. If $a=1$ then the Taylor expansion is given by

$$
R(u(x)+h(x))-R(u)=L_{1}(h)+L_{2}(h, h)+\|h\|^{2} \rho(h),
$$

where $\rho(h) \rightarrow 0$, as $h \rightarrow 0$.

A necessary condition of $u^{*}$ to be a minimum point of the functional $R(u)$ is

$$
\begin{aligned}
\varphi^{\prime}(0) & =L_{1}(h) \\
& =2 \int_{\Omega}\left[m^{\prime}\left(u^{*}\right) m^{\prime \prime}\left(u^{*}\right)\left|\nabla u^{*}\right|^{2} h+\left(m^{\prime}\left(u^{*}\right)\right)^{2} \nabla u^{*} \cdot \nabla h\right] d x=0,
\end{aligned}
$$

for every $h$ in a neighbourhood of $u^{*}$. According to the E-L equation the solution $u^{*}$ must satisfy

$$
m^{\prime}\left(u^{*}\right) \neq 0,
$$


otherwise the trivial solution $R\left(u^{*}\right)=0$ is obtained. Differentiating $\varphi^{\prime}(a)$ and rewriting the E-L equation using condition (5.20) we obtain $L_{2}(h, h)$ as

$$
\begin{aligned}
\frac{1}{2} L_{2}(h, h)=\int_{\Omega} & {\left[m^{\prime}\left(u^{*}\right) m^{\prime \prime \prime}\left(u^{*}\right)-3\left(m^{\prime \prime}\left(u^{*}\right)\right)^{2}\right]\left|\nabla u^{*}\right|^{2} h^{2} d x } \\
+ & \int_{\Omega}\left(m^{\prime}\left(u^{*}\right)\right)^{2}|\nabla h|^{2} d x .
\end{aligned}
$$

Since $L_{2}(h, h)>0$ implies a minimum, we consider the first integral. Assume $m \in \mathcal{C}^{3}$ and $u \in \mathcal{C}^{1}$, then there is an upper bound $C_{M}>0$ such that

$$
\left.\left|\left[m^{\prime}\left(u^{*}\right) m^{\prime \prime \prime}\left(u^{*}\right)-3\left(m^{\prime \prime}\left(u^{*}\right)\right)^{2}\right]\right| \nabla u^{*}\right|^{2} \mid \leq C_{M} .
$$

Let $\varepsilon>0$ and $B_{\varepsilon}$ be a set defined by

$$
B_{\varepsilon}=\left\{h, \nabla h \in L^{2}(\Omega):\|h\|_{L^{2}(\Omega)}^{2} \leq \varepsilon^{2} / C_{M}, \quad\|\nabla h\|_{L^{2}(\Omega)}^{2} \geq \varepsilon\right\} .
$$

Given that $h \in B_{\varepsilon}$, then the first integral of $L_{2}(h, h)$ reads

$$
\int_{\Omega}\left[m^{\prime}\left(u^{*}\right) m^{\prime \prime \prime}\left(u^{*}\right)-3\left(m^{\prime \prime}\left(u^{*}\right)\right)^{2}\right]\left|\nabla u^{*}\right|^{2} h^{2} d x \geq-C_{M} \int_{\Omega} h^{2} d x \geq-\varepsilon^{2} .
$$

Since $h \in B_{\varepsilon}$

$$
\int_{\Omega}\left(m^{\prime}\left(u^{*}\right)\right)^{2}|\nabla h|^{2} d x \neq 0 .
$$

By the mean value theorem of calculus there exists a $\xi \in \Omega$ such that $m^{\prime}\left(u^{*}(\xi)\right) \neq 0$ and

$$
\int_{\Omega}\left(m^{\prime}\left(u^{*}\right)\right)^{2}|\nabla h|^{2} d x=m^{\prime}\left(u^{*}(\xi)\right)^{2}\|\nabla h\|_{L^{2}(\Omega)}^{2}
$$

Hence

$$
\begin{aligned}
L_{2}(h, h) & \geq 2 \int_{\Omega}\left(m^{\prime}\left(u^{*}\right)\right)^{2}|\nabla h|^{2} d x-2 \varepsilon^{2} \geq 2\left(m^{\prime}\left(u^{*}(\xi)\right)\right)^{2}\|\nabla h\|_{L^{2}(\Omega)}^{2}-2 \varepsilon^{2} \\
& >2 \varepsilon\left[\left(m^{\prime}\left(u^{*}(\xi)\right)\right)^{2}-\varepsilon\right]>0
\end{aligned}
$$

since $h \in B_{\varepsilon}$ it is always possible to choose $\varepsilon<\left(m^{\prime}\left(u^{*}(\xi)\right)\right)^{2}$, which is the sufficient condition for $u^{*}$ to be a local minimum of $R(u)$. And the theorem follows.

\section{Selection of mapping function}

Inspired by the application of medical visualization, let $m: \mathbb{R} \rightarrow[0,1]$ be the transfer function $m \in \mathcal{C}^{3}$ computed from two user defined thresholds $u(x)=u_{1}$ to $u(x)=u_{2}$. Then the sigmoid function

$$
m(u(x), a, b)=(1+\exp (-(u(x)-b) / a))^{-1},
$$

defines the window of interest where $a=\left(u_{2}-u_{1}\right) / 4$ is the steepness and $b=$ $\left(u_{1}+u_{2}\right) / 2$ defines the offset. 
To show that condition $(5.14)$ is satisfied for 5.28 define, for notational convenience, a shorthand notation for the exponential function:

$$
e(u)=\exp ((b-u) / a)
$$

then, differentiating $m(u)$ up to third order gives

$$
\begin{aligned}
m(u) & =(1+e(u))^{-1} \\
m^{\prime}(u) & =-(1+e(u))^{-2} e^{\prime}(u), \\
m^{\prime \prime}(u) & =2(1+e(u))^{-3}\left(e^{\prime}(u)\right)^{2}-(1+e(u))^{-2} e^{\prime \prime}(u), \\
& =(1+e(u))^{-2}\left(2(1+e(u))^{-1}\left(e^{\prime}(u)\right)^{2}-e^{\prime \prime}(u)\right),
\end{aligned}
$$

and

$$
\begin{aligned}
m^{(3)}(u)=- & 6(1+e(u))^{-4}\left(e^{\prime}(u)\right)^{3}+2(1+e(u))^{-3} 2 e^{\prime}(u) e^{\prime \prime}(u) \\
& +2(1+e(u))^{-3} e^{\prime}(u) e^{\prime \prime}(u)-(1+e(u))^{-2} e^{(3)}(u) \\
=(1 & +e(u))^{-2}\left(-6(1+e(u))^{-2}\left(e^{\prime}(u)\right)^{3}\right. \\
& \left.+6(1+e(u))^{-1} e^{\prime}(u) e^{\prime \prime}(u)-e^{(3)}(u)\right),
\end{aligned}
$$

where

$$
e^{\prime}(u)=-\frac{1}{a} e(u), \quad e^{\prime \prime}(u)=\frac{1}{a^{2}} e(u), \quad \text { and } \quad e^{(3)}(u)=-\frac{1}{a^{3}} e(u) .
$$

Now, express the derivatives of $m(u)$ in $e(u)$ such that

$$
\begin{aligned}
m(u) & =(1+e)^{-1}, \\
m^{\prime}(u) & =\frac{1}{a}(1+e)^{-2} e, \\
m^{\prime \prime}(u) & =\frac{1}{a^{2}}(1+e)^{-2}\left(2(1+e)^{-1} e^{2}-e\right), \\
m^{(3)}(u) & =\frac{1}{a^{3}}(1+e)^{-2}\left(6(1+e)^{-2} e^{3}-6(1+e)^{-1} e^{2}+e\right) .
\end{aligned}
$$

Observe that $m^{\prime}(u)>0$ for all $u$, thus for this choice of mapping function the next result shows that the sufficient condition in (5.14) is satisfied.

The lower bound of $\left(m^{\prime}\left(u^{*}\right)\right)^{2}$ is given by

$$
\left(m^{\prime}\left(u^{*}\right)\right)^{2}=\frac{1}{a^{2}} \frac{e^{\frac{2\left(b-u^{*}\right)}{a}}}{\left(1+e^{\frac{b-u^{*}}{a}}\right)^{4}} \geq \frac{1}{a^{2}} \frac{e^{\frac{2(b-1)}{a}}}{\left(e^{\frac{b}{a}}+e^{\frac{b}{a}}\right)^{4}} \geq \frac{1}{a^{2} 16 e^{\frac{2(b+1)}{a}}},
$$

and a more strict condition than (5.14) is given by (5.27). This results in the final condition $\left(16 a^{2} e^{\frac{2(b+1)}{a}}\right)^{-1}>\varepsilon$. 


\subsection{Non-linear total variation}

Due to the popularity and the noise suppression properties of the total variation scheme [79] for image denoising it is of interest to also investigate its formulation expressed using the mapping function, TVm. An evaluation of TVm is presented in section 13.3 .

It is straightforward to extend the total variation formulation to include a mapping function: set $p=1$ in Proposition 2 to obtain

$$
R(u)=\int_{\Omega}|\nabla m(u)| d x .
$$

The corresponding E-L equation of (5.40) is obtained from Theorem 2 First define the tensor $W(\nabla m(u)) \in \mathbb{R}^{2 \times 2}$ as

$$
W(\nabla m(u))=\frac{1}{|\nabla m(u)|} I,
$$

then the differentiation with respect to each component of $W(\boldsymbol{s})=W(\nabla m(u))$ result in the expressions

$$
\begin{aligned}
& W_{s_{1}}=-\frac{1}{|s|^{3}} s_{1} I=-\frac{m^{\prime}(u)}{|s|^{3}} u_{x} I \\
& W_{s_{2}}=-\frac{1}{|s|^{3}} s_{2} I=-\frac{m^{\prime}(u)}{|s|^{3}} u_{y} I
\end{aligned}
$$

that yields the tensor ${ }_{\nabla u} W_{\boldsymbol{s}}(\nabla m(u))$ :

$$
\begin{aligned}
{ }_{\nabla u} W_{\boldsymbol{s}}(\nabla m(u)) & =\left(\begin{array}{c}
\nabla^{t} u W_{s_{1}} \\
\nabla^{t} u W_{s_{2}}
\end{array}\right)=-\frac{m^{\prime}(u)}{|s|^{3}}\left(\begin{array}{cc}
u_{x}^{2} & u_{x} u_{y} \\
u_{x} u_{y} & u_{y}^{2}
\end{array}\right) \\
& =-\frac{1}{\left(m^{\prime}\right)^{2}|\nabla u|^{3}} \nabla u \nabla^{t} u=-\frac{1}{\left(m^{\prime}\right)^{2}|\nabla u|^{3}} \nabla u \nabla^{t} u .
\end{aligned}
$$

Since $W(\nabla m(u))$ in 5.41 is symmetric, Corollary 2 in chapter 4 is applicable with $S=2 W+m^{\prime}{ }_{\nabla u} W_{\boldsymbol{s}}(\nabla m(u))$ and we get

$$
\begin{aligned}
m^{\prime} \operatorname{div}\left(m^{\prime} S \nabla u\right) & =m^{\prime} \operatorname{div}\left(m^{\prime}\left(2 \frac{I}{|\nabla m(u)|}-m^{\prime} \frac{1}{\left(m^{\prime}\right)^{2}|\nabla u|^{3}} \nabla u \nabla^{t} u\right) \nabla u\right) \\
& =m^{\prime} \operatorname{div}\left(2 \frac{\nabla u}{|\nabla u|}-\frac{\nabla u}{|\nabla u|^{3}}|\nabla u|^{2}\right) .
\end{aligned}
$$

Performing the final cancellation and subtraction, results in the E-L equation

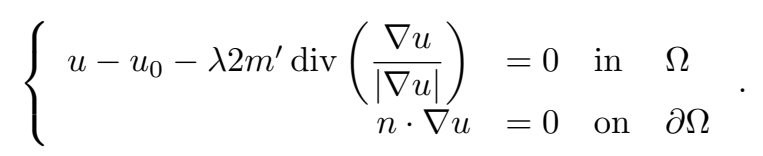

The standard total variation formulation is obtained by setting $m(u)=u$, which yield $m^{\prime}(u)=1$. Thus the standard methods are obtained as special cases of 
our presented framework. The introduction of the (possibly non-linear) mapping function adds an additional parameter which controls the amount of filtering based on some prior knowledge of the image value-range.

Note that, in contrast to targeted iterative filtering 5.10 , the sign of negative mapping functions does not cancel in (5.46) (cf. Remark 1). Therefore it is necessary that $m^{\prime}(u) \geq 0$ so that the solution is well-defined. 


\section{Chapter 6}

\section{Prior information in non-linear image filtering}

This chapter introduces a framework that can be used to drive the filtering processes based on probability density estimates. The material is adapted from our work [11. The main novelty lies in how to exploit an oversegmentation map, and from it, benefit from estimated density functions to drive the diffusion process presented in chapter 4

\subsection{Motivation}

Considering the image formation process, the basic principle is to gather incident light on photosensitive sensor elements. This is followed by sophisticated algorithms to transform electric charges on the sensor elements into an image that can be visualized on a monitor. It is commonly accepted that incident light on the photo sensor can be modelled as a stochastic process. Determining the parameters of the stochastic process is an ill-posed problem: we have the measurements in the image data, but we do not know the process which generated them. Therefore, one often assumes the principle of ergodicity, i.e., image data in a local neighbourhood can be used to model the uncertainty of the generative process (the rate at which incident light was captured on the photoelectric sensor element). In other words, by locally estimating the image data distribution we can say something about the stochastic process that initially generated the image data. This line of reasoning inspired the framework presenetd in this chapter. In short, we estimate homogeneous image segments to form an oversegmentation map of the image. Then, for each segment, we estimate the first and second moments from each segment. Since each segment is assumed to describe a locally homogeneous region, we use the principle of ergodicity to view each data point as a sample drawn from the estimated density function.

The following sections first describe the method via an example, followed by 

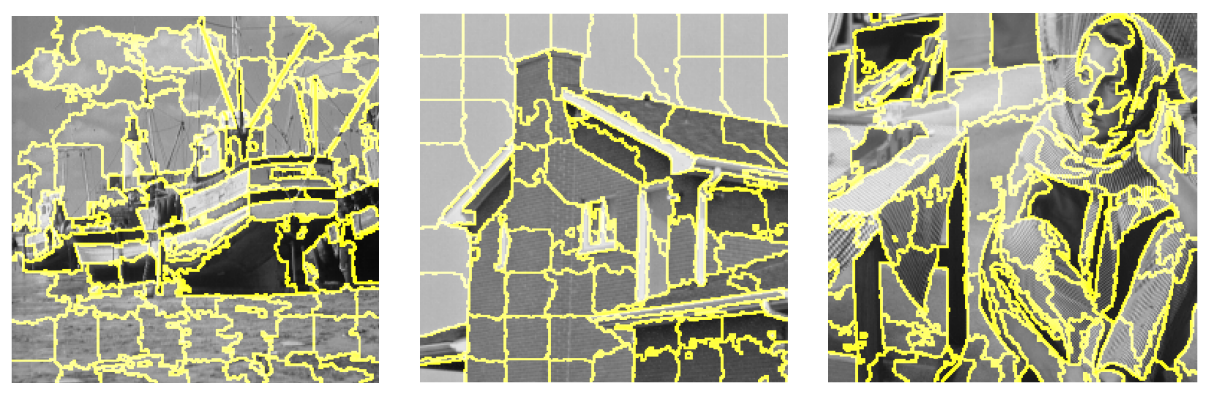

Figure 6.1: Typical segmentation maps produced by [66]. Notice how the segments are generally aligned with strong discontinuity boundaries in the image, while they adjust their shape and size to better approximate the homogeneous image regions.

the diffusion model "density driven diffusion" that utilises the estimated density functions previously described.

\subsection{Oversegmentation}

The first step of "density driven diffusion" involves dividing (oversegmenting) the image into distinct, non-overlapping regions, each containing a number of pixels with some consistent and perceptually meaningful set of properties (e.g., colour, texture or intensity). These regions form the segmentation map, which in turn is used for the estimation of the local PDFs necessary for the filtering stage. Since, as will discuss later, we estimate a simple, unimodal PDF in each region/segment, assume that each segment spans approximately a homogeneous intensity/colour image region and that the pixel values are i.i.d. across different segments. In other words, the segments do not span strong boundaries in the image data. Examples from such segmentation maps can be seen in figure 6.1 .

Obviously, not every oversegmentation method can produce segmentation maps with the above properties. There is a line of approaches called "superpixel" segmentation methods that can generate such maps. In particular, we have used the "relaxation labelling" method by [66], which adapts the size and shape of each segment (superpixel) according to the image structure, always favouring map configurations where each segment expands to fill a homogeneous image region, while avoiding crossing edges.

The advantage of using a segmentation map to drive the filtering process, as opposed to a uniform, local PDF estimation scheme can be seen in figure 6.2. In figure 6.2 (a) we can see the noisy synthetic input image. Figure 6.2 (c) shows the segmentation map produced by [66. Compare this with the randomized uniform grid in figure 6.2 (f). In figure 6.2 (d) and (g) we can see the "probability maps" estimated from the segmentations in figure 6.2 (c) and (f) respectively. A probability map illustrates the diffusion strength as determined by the estimated PDFs. Red indicates strong diffusion, desirable in homogeneous image regions. Blue indicates very weak diffusion and that should be along strong image edges. 


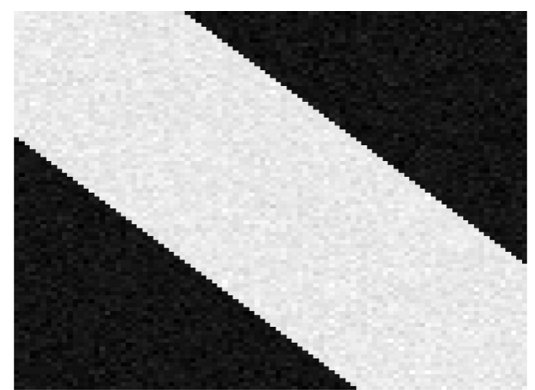

(a) Noisy

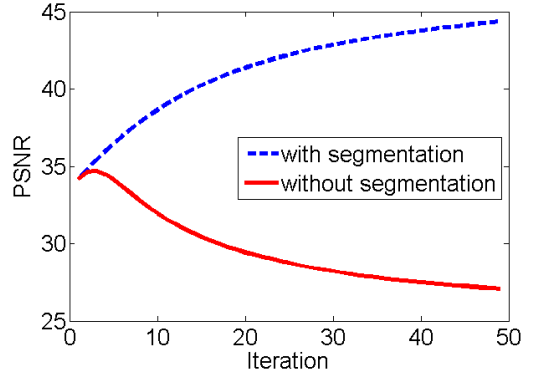

(b) PSNR comparison

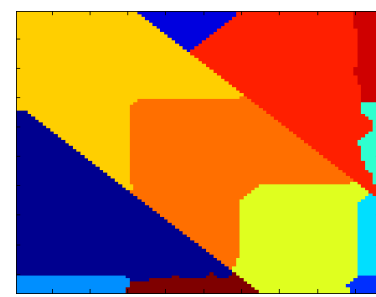

(c) Segmentation 66.

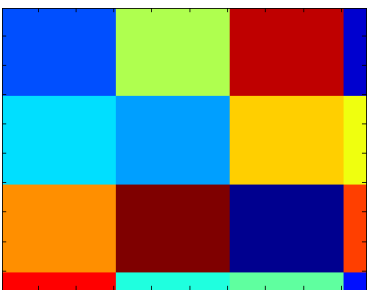

(f) Random grid

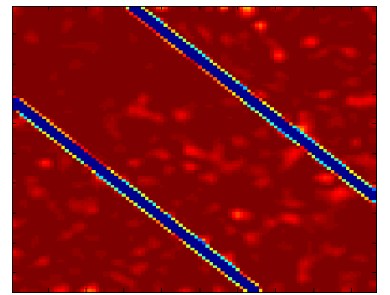

(d) Probability map

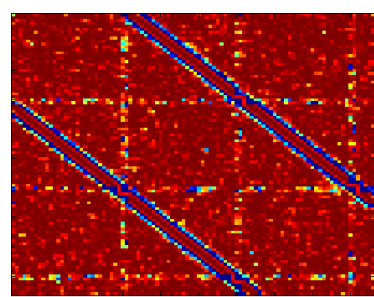

(g) Probability map

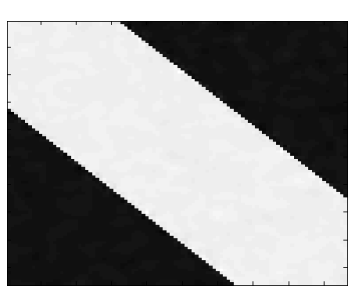

(e) Result

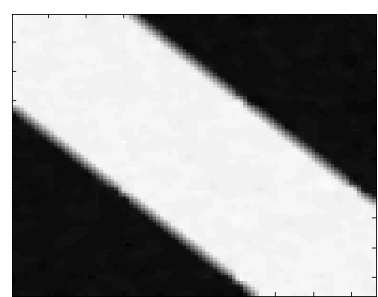

(h) Result

Figure 6.2: Example of applying the proposed filtering method on an synthetic image driven by an accurate segmentation map, compared to a map initialized with random labels. See section 6.2 for more details.

Note that the probability map in figure 6.2 (c) shows a more desirable diffusion behaviour leading to the very good denoising result in figure 6.2 (e). This is because the unimodal local PDFs that we have estimated in each segment from figure 6.2 (c) are good approximations to the true pixel distributions. Contrast this with the probability map figure 6.2 (g) estimated from the non structure preserving segmentation in figure 6.2 (f). Due to the poor estimation of the local PDFs in that case, the probability map is noisy, and does not reduce the filtering strength sufficiently along edges. As expected the resulting image in figure 6.2 (h) contains the same artefacts, and also most of the edge information has been lost. We can further see the quantitative difference between the two examples in terms of the PSNR plots in figure 6.2 (b). 


\subsection{Density driven diffusion}

The proposed method density driven diffusion, D3, consists of three basic steps. First we generate a segmentation map from a given noisy image. That is, we oversegment the image, obtaining a number of segments (image regions) that ideally exhibit two important properties: i) they are homogeneous (e.g., in terms of colour, texture, intensity and so on) and ii) they obey image boundaries, i.e., they align themselves along strong discontinuity boundaries in the image, instead of crossing them.

The segmentation map is utilised in the next step, which is the estimation of a number of density functions (PDFs), each describing the distribution of pixel values in each segment. We finally exploit the estimated PDFs in order to drive the filtering process in the third and final step, and thereby obtain the denoised image. Such a scheme takes advantage of the structure preserving properties of the oversegmentation and thus filters more heavily in regions of near-uniform colour/intensity (i.e., within each segment), while reducing the amount of filtering near edges (i.e., between different segments). As a result, D3 is more powerful than the simple linear diffusion schemes.

In the following sections, we describe in more detail the main components of our approach, starting with the density-driven filtering scheme, and conclude with a discussion of the PDF estimation process. An overview of the method is illustrated in figure 6.3 .

\subsubsection{The filtering scheme}

In the motivation of the proposed filtering scheme assume that content in a local neighbourhood adheres to the same stochastic process described by the random variable $U$. Then let $m(u)$ be the probability of a sample $u(x, y)$ belonging to this process. The PDF associated with the cumulative distribution function (CDF) $m(u)$ is then the non-negative function $m^{\prime}(u)$. The interpretation of $m(u)$ as a function which estimates domain-dependent information, i.e., local image statistics, leads to a natural application of Theorem 2 in chapter 4. In this case we consider the case when we do not have a tensor-valued diffusion, i.e., we have the functional

$$
E(u)=\int_{\Omega}\left(u-u_{0}\right)^{2} d x+\lambda \int_{\Omega}|\nabla m(u)|^{2} d x
$$

The interpretation of the smoothness term is that by minimizing the gradient of the density function, its variance is reduced, i.e., the distribution is sharpened. In terms of noise estimation, this implies that the samples belonging to the new random variable describe the sharpened distribution and more accurately reflects the true value distribution of the signal.

The corresponding Euler-Lagrange equation was given in 5.10 and can also be obtained by using Theorem 2 with $W(\nabla m(u))=I$ where $I$ is the identity matrix. 


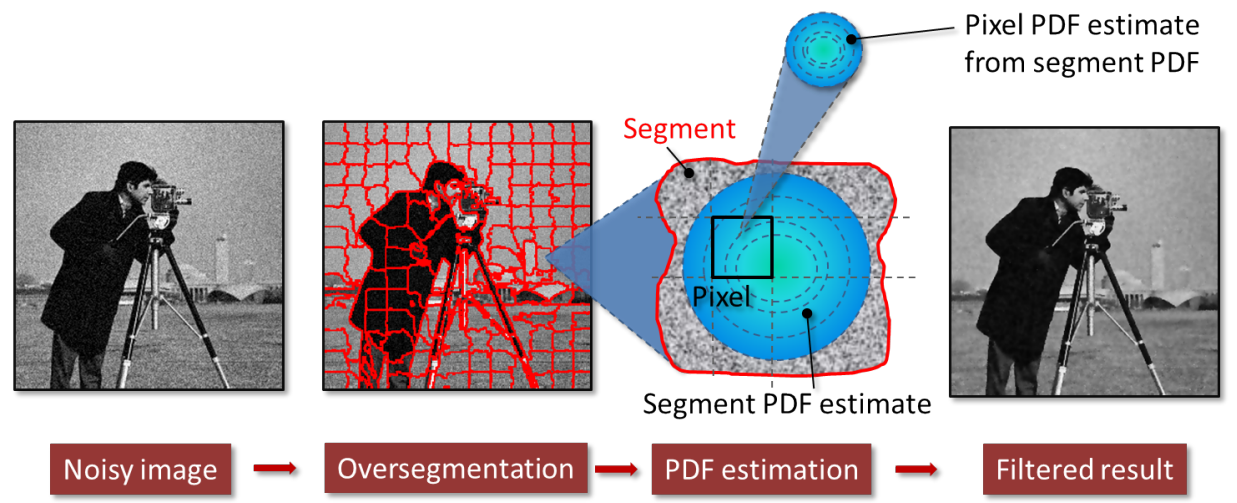

Figure 6.3: Overview of the three main components of our approach. First the noisy image is oversegmented into multiple homogeneous regions (segments). Then local PDFs are estimated using the segments' information. The estimated PDFs drive the filtering process, resulting in the final denoised image.

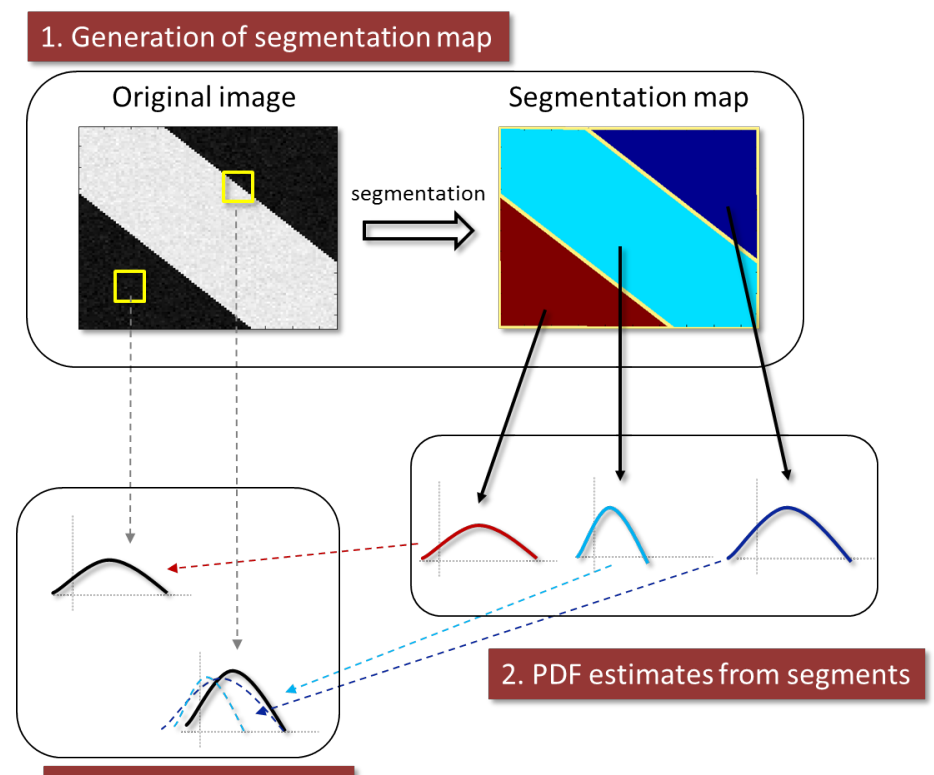

3. Local PDF estimates

Figure 6.4: Overview of the estimation of density functions in homogeneous regions giving rise to a single local density estimate. On the other hand, in textured regions they will give rise to mixture models. The number of mixture components are given by the number of spanned segments, and since the distributions are independent inside each segment, the mixture fitting is trivially determined. 


\subsubsection{Estimating Density Functions}

For every segment $s \in \mathcal{S}$, where $\mathcal{S}$ is the set of segments, found by the oversegmentation step, we fit a Gaussian distribution with $\mu_{s}$ and $\sigma_{s}^{2}$ being the mean value and the variance of the value distribution. Due to the properties of the oversegmentation it is reasonable to assume that the distributions in $\mathcal{S}$ are independent. Therefore, let the derivative of the mapping function in the E-L equation 5.10 be the product of $n$ distributions in a spatial neighbourhood $\mathcal{L}$ at a pixel with location $(x, y) \in \Omega$ then

$$
m^{\prime}(u)=\prod_{i \in \mathcal{L}} m_{i}^{\prime}(u)
$$

where $m_{i}^{\prime}(u)=\mathcal{N}\left(u ; \mu_{i}, \sigma_{i}^{2}\right)$. Figure 6.4 shows this process on a synthetic image for two cases; first for a homogeneous region where a single PDF is estimated and second in the case when a mixture of two regions gives rise to a corresponding mixture model. The corresponding CDF of a segment is given by the error function

$$
m_{s}(u)=\frac{1}{2}\left(1+\operatorname{erf}\left(\frac{u-\mu_{s}}{\sigma_{s} \sqrt{2}}\right)\right) .
$$

Note that the interpretation of the CDF is that it is a non-linear function which adaptively selects a range of intensity values corresponding to the segment's value distribution. The difference to non-linear diffusion as defined by Perona and Malik 72 is that $m^{\prime}(u)$ is selected by the image value distribution rather than an adhoc function operating on the gradient domain. In practice we are never required to compute the CDF since it does not appear in the E-L equation (cmp. (5.10)).

The density driven diffusion, framework can also be interpreted in relation to the methods mean shift denoising [26] (MS), non-local means [22] (NLM) and bilateral filtering 90 .

First, the mean shift method estimates density function in a feature space defined by, e.g., opponent colours components. Thus the density estimate for a mode in this feature space can be viewed as joining all similar (spatially connected) patches found by the oversegmentation map in D3, and then estimating the density function. Whereas mean shift directly modifies the estimated density functions, D3 uses the density estimates as a prior to achieve robust filtering.

Bilateral filtering use a fixed similarity function (often Gaussian function) to estimate structure similarity in a local neighbourhood. In contrast, D3 estimates a density function, which can be thought of a similarity function for a local segment in the oversegmentation map. Therefore, D3 achieves an adaptive denoising scheme, without the requirement of determining the size of the similarity window, since this is implicitly done via the oversegmentation map.

The non-local means algorithm considers both spatial and photometric similarity functions (or windows) to adaptively denoise the image data. In the most general formulation, the NLM similarity functions are defined over the domain of the image. However, due to computational reasons, the spatial extent of the similarity functions are limited. Therefore in contrast to D3, NLM considers local regions of fixed size. The second major difference to D3 is that NLM performs patch-based averaging within the spatial similarity window, this is not done in D3. 


\section{Chapter 7}

\section{Channel-based image processing}

This chapter explores a novel approach to domain-dependent non-linear diffusion. The idea is to exploit the channel representation (introduced and defined below) to drive the filtering scheme. The material presented in this chapter is an adaptation of our work [47. The aim is to construct a filtering method which handles both Gaussian as well as impulse (or salt-and-pepper) noise in a unified framework. In the context of this thesis, the adaptation of the channel representation formulation can be explained in relation to Theorem 2 2 Previously the mapping function described the visualization window (section 5.2 in medical imaging or estimates densities from local neighbourhoods (chapter 6). In this chapter, the mapping function is described by the basis-functions of the channel representation.

\subsection{Motivation}

Crucial for the performance of non-linear diffusion is an adaptation of the diffusivity, i.e., the local smoothing strength, to image structure. Typically, the strength of the image structure is measured as the Euclidean norm of the local image gradient. It is transformed into a diffusivity by means of an edge-stopping function, assigning small diffusivities to locations with high gradient and vice versa. The exact choice of the edge-stopping function has been shown to be equivalent to the choice of error norm in robust statistics [18] and can be learned from image statistics [100, 77.

If outliers are present in the data, e.g., as salt-and-pepper noise, or dropouts, other reconstruction methods than diffusion are usually applied, able to remove outliers completely, e.g., median filtering (see, e.g., [41]), or channel smoothing [34] selecting the maximum mode of the local value distribution. Channel smoothing (CS) averages not only by applying a spatial window, but also windows in the value domain. However, the value domain window is not centred at the value of the currently processed pixel, in contrast to bilateral filtering [90, but centred at the maximum of the local value distribution. By this, it removes clear outliers and 
interpolates from neighbours. If Gaussian noise is present in the data, also a part of the inliers are lost due to the value domain window. This since the data is not considered in the value reconstruction. Consequently the reconstructed grey value is less efficiently denoised than if all inliers were considered.

The rational behind our approach is the observation: robust diffusion schemes such as PM diffusion, which can be derived from a regularization term penalising the gradient of a signal, reduces the smoothing process at edges, i.e., high gradient values. An outlier may erroneously be detected as edge and by this not smoothed away. Not considering the outlier in the penalising term thus is still expected to result in high smoothing strengths at outlier positions, reducing their visibility.

The natural test case for such a regularization is its use without a data term, i.e., image diffusion not regularization. Further, as the robustness to outliers is of interest, one natural application is image reconstruction in the presence of mixed noises.

We use mixtures of Gaussian and impulse-like noise. The motivation for introducing the channel based regularization in this part of the thesis is that we may consider the channel representation as a model for the stochastic behaviour of the image acquisition processes as was exemplified in chapter 6. It is not expected that the introduced scheme will give the best grey value image reconstruction and the experiments show that state-of-the-art denoising schemes do a better job here. However, this additional robustification by channel smoothing can easily be transferred to application domains where diffusion schemes are used in practice.

The core idea of this approach is to drive a non-linear diffusion process using the CS result as image structure information. CS alone gives poor denoising results for medium to high Gaussian noise levels. In this case, non-linear diffusion can yield higher signal-to-noise ratio (SNR) as it can average over all available data. However, standard non-linear diffusion which is driven by the local gradient stops at clear outliers, not suppressing them. CS removes outliers while preserving edge location and (roughly) edge strength. Thus driving a non-linear diffusion by CS allows for oversmoothing, not removal of outliers. We therefore expect to gain SNR compared to plain CS when a considerable amount of Gaussian noise is present and still suppress outliers such that they are less visible in the image.

The following section gives a brief introduction to the framework of channel smoothing before introducing the proposed channel-based regularization terms.

\subsection{Channel smoothing}

In channel smoothing 34 the channel vectors are spatially averaged using a Gaussian kernel $w$ :

$$
\tilde{B}_{i}(x, y)=w * B_{i}(u(x, y))
$$

where $B_{i}(x, y)$ are the basis functions (see 2.2.12). Reconstructing a value $u$ from the channel representation can be done using a linear combination of all channel vector components yielding a linear decoding. To obtain a robust decoding scheme only a subset of the channel components is used 34. Often a window of size 3 


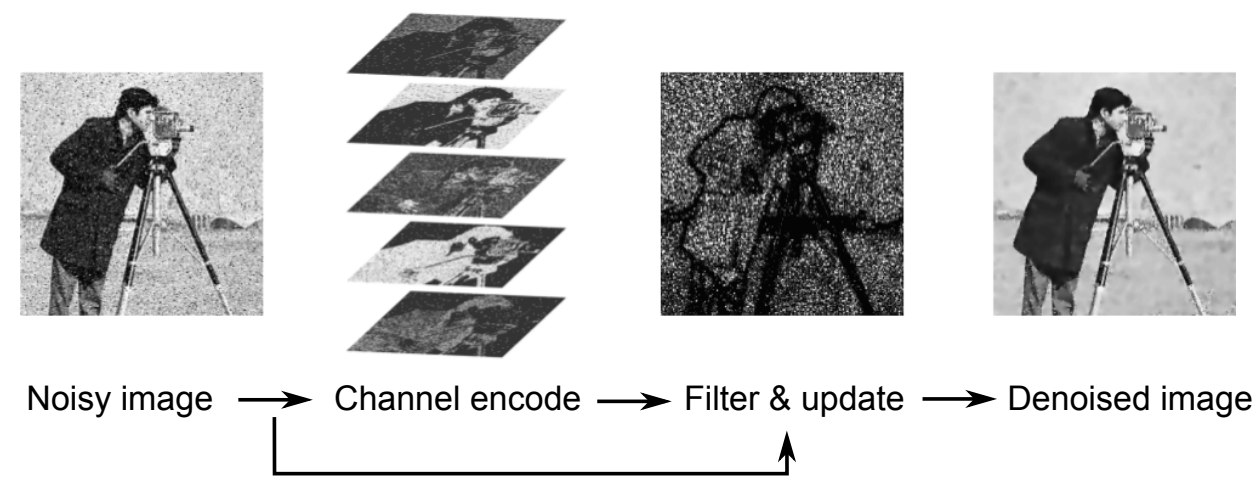

Figure 7.1: Overview of the channel-based regularization update scheme. Contrast is increased in "Filter \& update" for a better visualization.

around the maximum mode location of the channel vector is computed, i.e.

$$
l=\underset{k}{\operatorname{argmax}} \sum_{i=k-1}^{k+1} \tilde{B}_{i}(x, y),
$$

with $k=2, \ldots, N-1$. Then the robust decoding scheme reads

$$
\hat{u}(x, y)=\sum_{i=l-1}^{l+1} c_{i} \tilde{B}_{i}(x, y),
$$

where it is assumed that the coefficients $\tilde{B}_{i}(x, y)$ sum up to one, if not, they are normalized to do so. Note that the choice of $l$ in $(7.2)$ depends on continuously differentiable basis functions such that the local maxima are continuous functions of the input values. The order of local maxima might change depending on the input, but this is desired since this reflects, e.g., the non-stationarity at edges.

In the next section we introduce an energy functional combining the framework of diffusion filtering and channel representation. We first derive linear channelbased diffusion (LCD) before extending it to the non-linear case (NLCD).

\subsection{Isotropic channel-based regularization}

The schematic diffusion approach is illustrated in figure 7.1. The noisy image is decomposed into its corresponding channel representation. Within the channel space we determine a local neighbourhood which best represents the data sample in the image space. This guides the diffusion process such that both Gaussian and impulse noise are reduced, indicated by the white spots in the "Filter \& Update" step. Black indicates no update, so important structure and edges in the image are preserved.

Clearly, the diffusion process cannot remove outliers from data, but strongly smooth impulse-like outliers. It is therefore well adapted to improving the visual 
impression and preserving the structure of reconstructed images, measured by SSIM 94 in our experiments. Due to the mean-preserving property of diffusion filtering, bias-free improvement of the reconstructed data values can only be hoped for, when zero-mean noise is present.

In order to enable diffusion methods to oversmooth impulse noise, the regularization term $R(u)$ is defined using the channel representation as described in previous sections. This means to encodes $u$ into the channel space using $(7.3)$ thus leading to the regularization term

$$
R(u)=\int_{\Omega}\left|\nabla \sum_{i=l-1}^{l+1} c_{i} \tilde{B}_{i}(x, y)\right|^{2} d x
$$

where $l$ is defined in $\sqrt{7.2}$ and $\tilde{B}$ are the smoothed channel weights in 7.1 . To simplify notation, define

$$
\sum_{i=l-1}^{l+1} c_{i} \tilde{B}_{i}(x, y)=c^{t} \tilde{B}
$$

where the 3-box windowing is implicitly included in $\tilde{B}$. The integrand of $R(u)$ can then be written in vector-matrix notation as $\left|\nabla\left(c^{t} \tilde{B}\right)\right|^{2}$ which yields

$$
R(u)=\int_{\Omega} c^{t}\left[\left(\partial_{x} \tilde{B}\right)\left(\partial_{x} \tilde{B}\right)^{t}+\left(\partial_{y} \tilde{B}\right)\left(\partial_{y} \tilde{B}\right)^{t}\right] c d x .
$$

To find the E-L equation of 7.6 it is necessary to compute its variational derivative and the line of calculations is analogous to previous chapters. However, in this part, to clarify the presentation, the $x$ - and $y$-component are treated separately. Hence for the $x$-component

$$
\begin{aligned}
& \partial_{u} R(u) v= \frac{\partial}{\partial \varepsilon}\left[\int_{\Omega} c^{t} \tilde{B}^{\prime}(u+\varepsilon v) \tilde{B}^{\prime}(u+\varepsilon v)^{t}\right. \\
&\left.\cdot\left(\partial_{x} u+\varepsilon \partial_{x} v\right)^{2} c d x\right]\left.\right|_{\varepsilon=0} \\
&=\int_{\Omega} c^{t}\left(\left.\frac{\partial}{\partial \varepsilon}\left[\tilde{B}^{\prime}(u+\varepsilon v) \tilde{B}^{\prime}(u+\varepsilon v)^{t}\right]\right|_{\varepsilon=0}\right) c\left(\partial_{x} u\right)^{2} \\
& \quad+2 c^{t}\left(\tilde{B}^{\prime}(u) \tilde{B}^{\prime}(u)^{t} \partial_{x} u \partial_{x} v\right) c d x \\
&=\int_{\Omega} c^{t}\left(\tilde{B}^{\prime \prime}(u) \tilde{B}^{\prime}(u)^{t}+\tilde{B}^{\prime}(u) \tilde{B}^{\prime \prime}(u)^{t}\right) c\left(\partial_{x} u\right)^{2} v d x \\
&-2 \int_{\Omega} \partial_{x}\left(c^{t} \tilde{B}^{\prime}(u) \tilde{B}^{\prime}(u)^{t} c \partial_{x} u\right) v d x .
\end{aligned}
$$

Similar result holds for the $y$-component. In (7.8) Green's formula was applied with Neumann boundary conditions to get rid of the $\partial_{x} v$ term, which cannot be determined.

Let

$$
S(u)=\tilde{B}^{\prime}(u) \tilde{B}^{\prime}(u)^{t},
$$




$$
\left(\begin{array}{cccccc}
0 & 0 & & \cdots & & 0 \\
0 & \ddots & & & & \\
& & * & * & * & \\
\vdots & & * & * & * & \vdots \\
& & * & * & * & \\
0 & & & \cdots & & 0
\end{array}\right)
$$

(a)

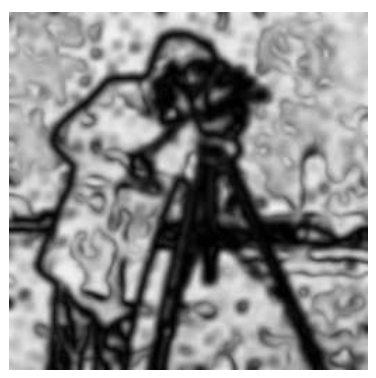

(b)

Figure 7.2: (a) Typical structure of $S(u)$ for a certain spatial position. The size of the matrix is equal to the number of channels used for encoding. The number of non-zero elements are given by the size of the decoding window and are located on the diagonal of $S(u)$. In this example, 3 channels are selected for decoding. (b) Structure of $c^{t} S(u) c$ for the 8 bit cameraman image and a Gaussian kernel $w$ with standard deviation 3. Black indicates low values close to 0 and white indicates high values close to 1 .

then by the definition of divergence and the equality

$$
\operatorname{div}\left(c^{t} S(u) c \nabla u\right)=c^{t} S^{\prime}(u) c \nabla^{t} u \nabla u+c^{t} S(u) c \Delta u,
$$

the variation of the energy in the direction of $v$ can be formulated as

$$
\partial_{u} R v=-\left[\operatorname{div}\left(c^{t} S(u) c \nabla u\right)+c^{t} S(u) c \Delta u\right] v .
$$

With the derived functional derivative we are able to obtain the PDE

$$
\left\{\begin{array}{rll}
\partial_{t} u-\lambda\left(\operatorname{div}\left(c^{t} S(u) c \nabla u\right)+c^{t} S(u) c \Delta u\right) & =0 & \text { in } \Omega \\
n \cdot \nabla u & =0 & \text { on } \partial \Omega
\end{array},\right.
$$

where $\lambda>0$. The matrix $S(u)$ is a symmetric matrix with entries as a block of size three centred around the main diagonal as shown in figure 7.2 (a). Since $S(u)$ is the outer product of the vector $\tilde{B}^{\prime}$, it is positive semi-definite. The scalar value $c^{t} S(u) c$ acts as a weight and results in a coefficient with large entries in homogeneous areas as the box decoding includes almost all relevant values. At edges the coefficient has small entries. At outlier positions the coefficient is still large (cmp. figure 7.2 (b)).

Observe that the left hand side of 7.13 , top, consists of two terms, where the left one has the usual form of non-linear diffusion with spatial varying diffusivity $c^{t} S(u) c$, and the right one the form of a diffusion term ignoring the spatial variation of $c^{t} S(u) c$. For linear decoding, channel smoothing breaks down to simple local averaging of $u$ and $c^{t} S(u) c=1$, independent of the variance of $w$ (see (7.1)), and 7.13 becomes plain linear diffusion $(3.9)$. Using robust decoding $c^{t} S(u) c$ becomes a spatially varying function and (7.13) a non-linear diffusion with reduced diffusivity at edges and high diffusivity in homogeneous regions as well as at outlier positions as illustrated in figure 7.2 (b). 


\subsection{Non-linear channel-based regularization}

In this section we extend the LCD to its non-linear pendant including a convex potential function $\Phi$. The motivation is to further control the filtering to preserve fine image details, similarly to the approach by Perona and Malik [2]. Including a potential function allows for further suppression of outliers as they will not appear in the structure estimation.

Let the regularization term be defined as

$$
R(u)=\int_{\Omega} \Phi\left(\left|\nabla\left(c^{t} \tilde{B}(u)\right)\right|\right) d x
$$

where $c^{t} \tilde{B}(u)$ is the channel smoothed version of the image $u$ as defined in 7.3 . By computing the variational derivative $\partial_{u} R v$ in a similar way as presented in the previous section we obtain

$$
\begin{aligned}
\partial_{u} R v & =\int_{\Omega} \Phi^{\prime}\left(\left|\nabla\left(c^{t} \tilde{B}(u)\right)\right|\right) \frac{1}{2} \frac{1}{\left|\nabla\left(c^{t} \tilde{B}(u)\right)\right|} \cdot \frac{\partial}{\partial \varepsilon}\left[\left|\nabla\left(c^{t} \tilde{B}(u+\varepsilon v)\right)\right|^{2}\right]_{\varepsilon=0} d x \\
& =\int_{\Omega} \frac{1}{2} \Psi c^{t} S^{\prime} c|\nabla u|^{2} v+c^{t} S c \Psi \nabla u \cdot \nabla v d x \\
& =\int_{\Omega}\left(\frac{1}{2} \Psi \nabla\left(c^{t} S c\right) \cdot \nabla u-\operatorname{div}\left(c^{t} S c \Psi \nabla u\right)\right) v d x
\end{aligned}
$$

where $S(u)=\tilde{B}^{\prime}(u) \tilde{B}^{\prime}(u)^{t}$ and

$$
\Psi=\frac{\Phi^{\prime}\left(\left|\nabla\left(c^{t} \tilde{B}(u)\right)\right|\right)}{\left|\nabla\left(c^{t} \tilde{B}(u)\right)\right|}
$$

Now, using the expansion

$$
\operatorname{div}\left(c^{t} S c \Psi \nabla u\right)=\Psi \nabla\left(c^{t} S c\right) \cdot \nabla u+c^{t} S c \operatorname{div}(\Psi \nabla u),
$$

in 7.15 we obtain the following PDE

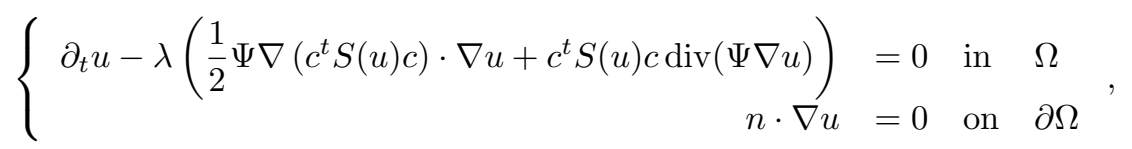

where $\lambda>0$ as before.

The terms in the parenthesis of (7.18) implement non-linear diffusion weighted by an additional factor. In the first term the diffusivity stems from the edge stopping function $\Psi$ and the channel representation acts as additional weight. In the second term the roles of $\Psi$ and $c^{t} S(u) c$ are exchanged. Furthermore, $\Psi$ is defined on the channel smoothed image $c^{t} \tilde{B}(u)$, so outliers are not present in the structure estimation of $\Psi$ and will be smoothed strongly. 


\section{Chapter 8}

\section{Gradient energy tensor}

A fundamental part of many image processing pipelines is to estimate the local orientation of image structures. Many techniques exists, and depend on image derivatives to capture the orientation information. Perhaps the most frequently used estimator is the structure tensor [39, 16]. Other alternatives include quadrature filters [55, 56, 43] and the gradient energy tensor [37.

Since the structure tensor and the gradient energy tensor (GET) are integral parts of the methods presented in this thesis, an extensive analysis of the tensors is presented that includes theoretical as well as geometrical interpretations. This chapter also presents applications of the gradient energy tensor for corner detection and optical flow estimation.

In chapter 14 the structure tensor in anisotropic diffusion is replaced with the gradient energy tensor. Empirical results show that the gradient energy tensor can significantly increase the achieved frames per second (fps) compared to the structure tensor to enable real-time image denoising.

\subsection{Definition of tensor}

The origin of the gradient energy tensor comes from the 1-dimensional energy operator, and in particular, from the observation that the energy of a signal can be computed from its squared magnitude [51]. The gradient energy tensor is symmetric, like the structure tensor, and it can be shown that GET is a phase invariant and orientation equivariant second order tensor [35, 70]. The GET tensor is lesser known in the computer vision community and it was first introduced by Felsberg and Köthe [37. The motivation for considering the GET in this work, as opposed to the structure tensor (2.34), is that the GET does not (necessarily) require a post-convolution of its tensor-components. Despite there being no postconvolution, GET is a rank 2 tensor which is real-valued and symmetric and it determines the directional energy distribution of the signal gradient. This allows us to easily use the tensor in our variational framework. 

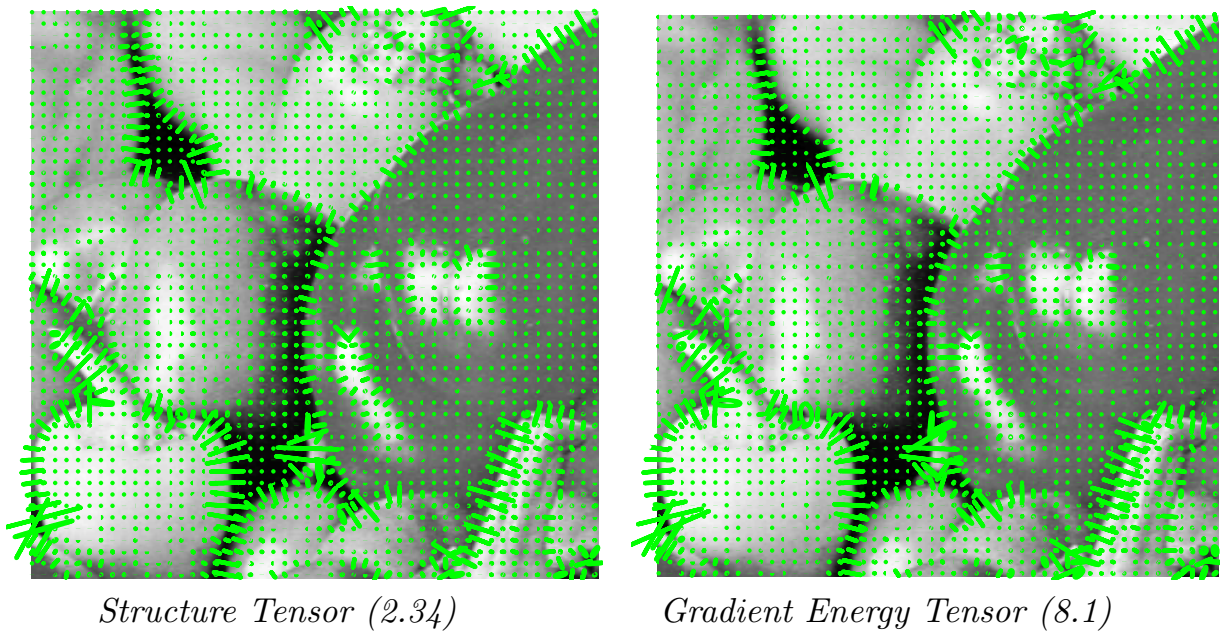

Figure 8.1: Illustration of the resulting tensor-fields. The structure tensor requires additional post-smoothing to form a rank-2 tensor compared to the gradient energy tensor which is defined without post-smoothing. Note that the size of the ellipses has been scaled for improved visualization.

The classical GET is defined in terms of the image data in $u$ 37]. An equivalent formulation of GET is to express it in terms of the image gradient. Let $H u=\nabla \nabla^{t} u$ be the Hessian and $\nabla \Delta u=\nabla \nabla^{t} \nabla u$, then we define the GET as

$$
G E T(\nabla u)=H u H u-\frac{1}{2}\left(\nabla u[\nabla \Delta u]^{t}+[\nabla \Delta u] \nabla^{t} u\right)
$$

Figure 8.1 shows the estimated orientations visualized as ellipses for both the structure tensor 2.34) and the gradient energy tensor 8.1. Note that due to the convolution operator, the structure tensor is not sensitive to structures smaller than the width of the averaging filter used to compute it (in this case the standard deviation of the Gaussian filter was set to 1). The presence of second and thirdorder derivatives in GET does makes it more sensitive to noise than the structure tensor. However, it allows us to capture orientation of structures not possible to detect using the structure tensor.

The positivity of the 1D energy operator was investigated by Bovik and Maragos [19] and the positivity of the 2D GET-tensor was considered by Felsberg and Köthe [37. In the two-dimensional case, the positivity of the operator is reflected in the sign of the eigenvalues. Let the components of the GET be $a, b$ and $c$, i.e.,

$$
G E T=\left(\begin{array}{ll}
a & b \\
b & c
\end{array}\right)
$$


then the entities $a, b, c$ of GET 8.1 read

$$
\begin{aligned}
& a=u_{x x}^{2}+u_{x y}^{2}-u_{x}\left(u_{x x x}+u_{x y y}\right) \\
& b=u_{x x} u_{x y}+u_{y x} u_{y y}-\frac{1}{2}\left(u_{x}\left(u_{y x x}+u_{y y y}\right)+u_{y}\left(u_{x x x}+u_{x y y}\right)\right), \\
& c=u_{y y}^{2}+u_{y x}^{2}-u_{y}\left(u_{y x x}+u_{y y y}\right) .
\end{aligned}
$$

The below result states that GET is positive semi-definite if the condition in Lemma 2 is satisfied.

Lemma 2 (Condition GET positive semi-definite). The GET is positive semidefinite if

$$
\operatorname{tr}(H u H u)-\nabla^{t} u \nabla \Delta u \geq \sqrt{l},
$$

where $l=\operatorname{tr}(G E T)^{2}-4 \operatorname{det}(G E T) \geq 0$.

Proof. Since GET is symmetric it has real eigenvalues. Thus by its eigenvalue decomposition it is sufficient to show that $\operatorname{tr}(G E T) \geq \sqrt{l}$ in order for GET to be positive semi-definite. $l$ is necessarily positive since $l=(a-c)^{2}+4 b^{2} \geq 0$.

In essence the GET-tensor can attain zero-values despite the gradient is not zero, also in some cases the eigenvalues may be negative. Due to the possible existence of negative eigenvalues it is convenient, for image diffusion applications, to define a modified $G E T$ denoted $G E T^{+}$. This asserts that the tensor is at least positive semi-definite, i.e.,

$$
\operatorname{GET}^{+}(\nabla u)=\left|\mu_{1}\right| v v^{t}+\left|\mu_{2}\right| w w^{t},
$$

where $v, w$ are the eigenvectors and $\mu_{1}, \mu_{2}$ eigenvalues of the GET respectively.

\subsection{Orientation estimation}

This section presents an empirical analyses of the effect of noise perturbation on the tensor orientation. To do so, consider the radially symmetric test-pattern shown in figure 8.2 (a). As a baseline for the evaluation the noise-free angles were computed and was also used to compute the test-pattern image. For each tensor and considered noise level, we compute the local neighbourhood's dominant distribution angle by projecting the tensor components on a vector $z$ in the complex plane 43,

$$
z=a-c+i 2 b .
$$

First we compare the mean absolute difference of the angular error for the two tensors in figure 8.3 (a) for the different noise levels. Figure 8.3 (b) presents the percentage of pixels that have an angular error smaller than 1 degree. It is evident that the two tensors are marginally different. However, as the noise level increase beyond 20 standard deviations of Gaussian noise the structure tensor performs better due to the post-convolution of the tensor components. At 0 and 10 standard deviations of noise, the difference is approximately $10 \%$. The third evaluation is a comparison of the histogram error quantized to 10 bins in the range 


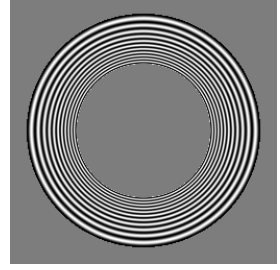

(a)

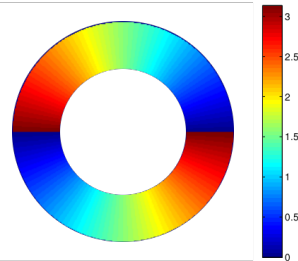

(b)

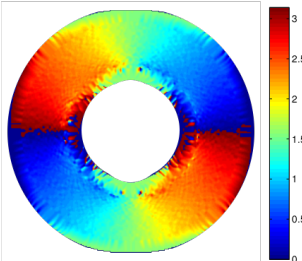

(c)

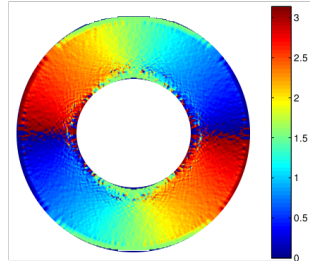

$(\mathrm{d})$

Figure 8.2: (a) Original, noise free circular pattern. (b) Ground truth orientation. Orientation in radians at 20 standard deviations of Gaussian noise, (c) Structure tensor and (d) Gradient energy tensor.

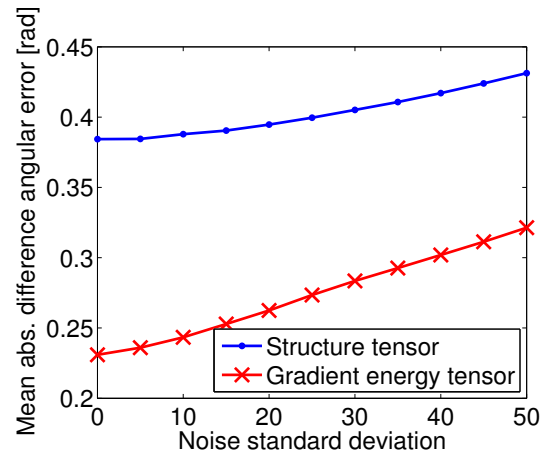

(a)

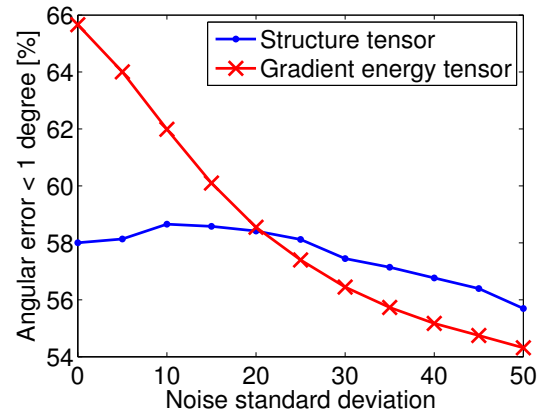

(b)

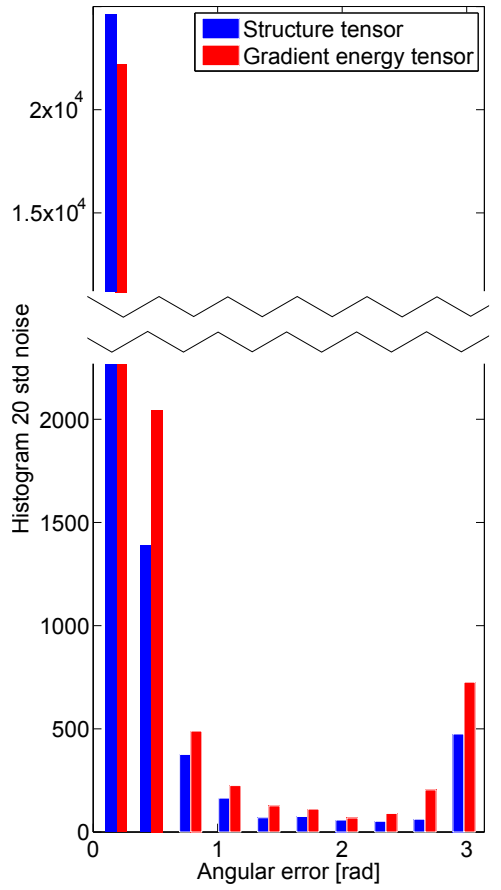

(c)

Figure 8.3: Results of sensitivity analysis of orientation estimation comparing the structure tensor and the gradient energy tensor. We used the radially symmetric pattern in figure 8.2 (a) for different noise levels. (a) shows the mean absolute angular error. (b) illustrates the percentage of pixels that has an angular error smaller than 1 degree. (c) shows the histogram of errors for the two tensors. 
$0-\pi$ at the intersection point seen in (b), i.e., at 20 standard deviations of noise. The histogram is shown in (c) and note that the error distribution is similar for the two tensors. For the smallest errors $(<0.5 \mathrm{rad})$ the gradient energy tensor has fewer errors than the structure tensor. However, considering the errors with larger magnitude, the gradient energy tensor has a marginally higher error rate.

The conclusion is that the gradient energy tensor does yield a worse angular estimate than the structure tensor with regards to the angular error distribution. However, this is expected since the energy tensor lacks a post-convolution of the tensor components to compensate for the noise. Nevertheless, considering that the difference between the two tensors' mean average angular error is small, as indicated in figure 8.3 (a), it suggests that the gradient energy tensor can be used in a diffusion framework, a claim that is further supported in chapters 9 and 14.

In the next section the gradient energy tensor is used for the applications of corner detection and optical flow estimation.

\subsection{Corner detection and optical flow}

This section presents the applicability of the gradient energy tensor for corner detection and optical flow.

\subsubsection{Corner detection}

The first application is corner detection using Good Features to Track [83]. The problem of corner detection is part of many image processing pipelines such as interest point detection and sparse optical flow. The good features to track framework detects corners by considering the eigenvalues of the structure tensor. If the structure tensor has two non-zero eigenvalues $\mu_{1,2}$, both larger than some threshold $\mu$ then the orientation tensor is necessarily invertible, i.e., the tensor is of rank 2. If the below condition is satisfied

$$
\min \left(\mu_{1}, \mu_{2}\right)>\mu
$$

where $\mu$ is often set to a fraction of the largest minimum eigenvalue then the neighbourhood contains a corner point.

The threshold was set to $\mu=0.01$ and figure 8.4 shows the 128 strongest detected corners for the structure tensor and the gradient energy tensor, respectively. We use a Gaussian kernel with standard deviation 1 for post-smoothing of the structure tensor components. The repeatability measure 68 is determined at $40 \%$ overlap (see figure 8.4 for the viewpoint dataset where the viewpoint angle has been changed from 20-60 degrees from the reference image 67. The repeatability measure is similar for the two tensors. Note that the gradient energy tensor, in this example, does not contain a post-smoothing of the tensor components.

Figure 8.4 shows the detected corner points in the reference image and for viewpoint changes of 20 and 40 degrees respectively. As illustrated in the two result images, the structure tensor and the gradient energy tensor based good features to track corner points are often identical and show very similar repeatability values. 


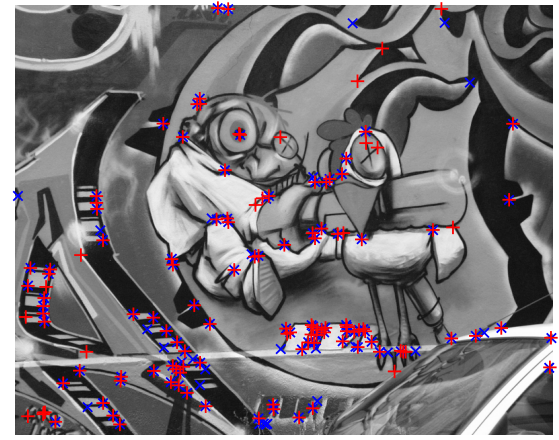

Reference

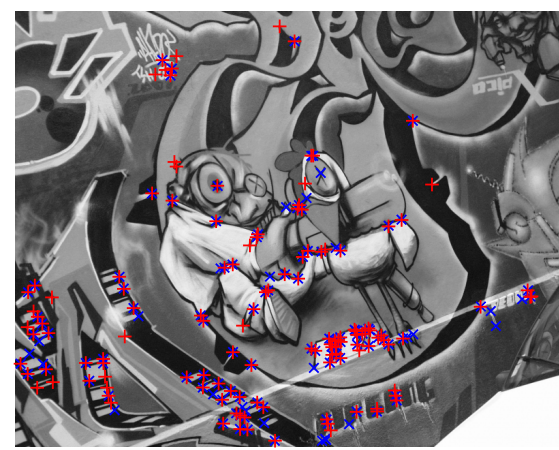

20 degrees viewpoint angle
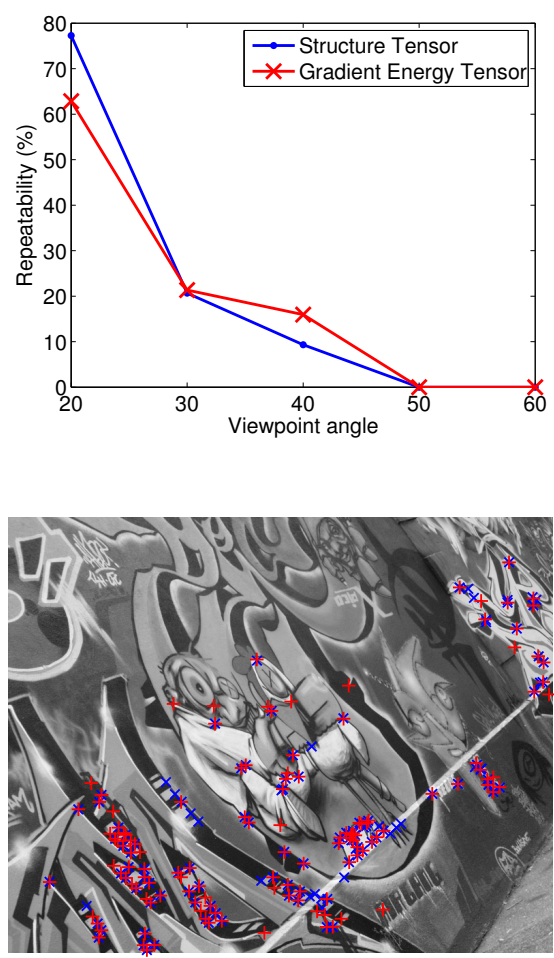

40 degrees viewpoint angle

Figure 8.4: Examples of corner detection for the structure tensor (with postsmoothing) and the gradient energy tensor (without post-smoothing) using good features to track. Both tensors detect corners accurately but not always the same corners.

\subsubsection{Optical flow}

The second application is to apply the gradient energy tensor to the original LucasKanade optical flow formulation 64 to compute a dense motion field. By minimizing the below energy functional the structure tensor appears as part of the minimizer

$$
E(u)=\int_{\Omega}[J(x+d)-I(x)]^{2} w(x) d x
$$

where $J$ and $I$ are two images of size $\Omega$ with some unknown displacement vector $d$. The minimizer of 8.10 is obtained by solving (see [89])

$$
\left[\int_{\Omega}\left[\nabla J(x) \nabla^{t} J(x)\right] w(x) d x\right] d=\int_{\Omega}[(J(x)-I(x)) \nabla J(x)] w(x) d x .
$$

The bracket in the left hand side of (8.11) is the structure tensor, $T$ in (2.34). Figure 8.6 shows the result when (8.11) is minimized with the structure tensor and 


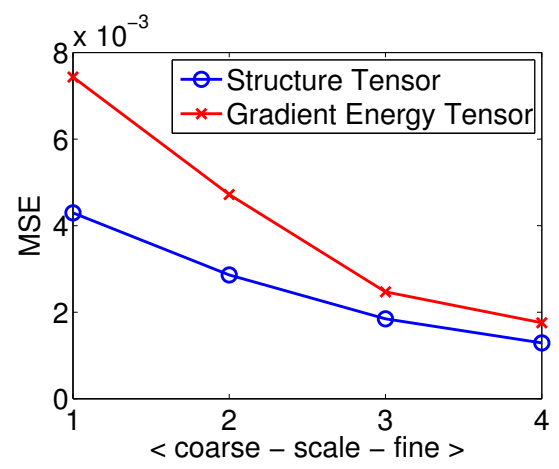

Backyard

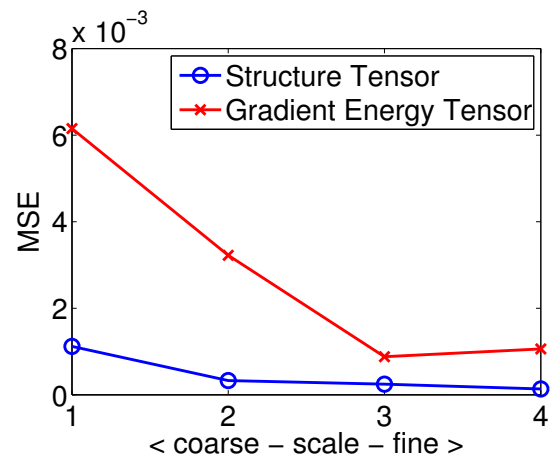

Wooden

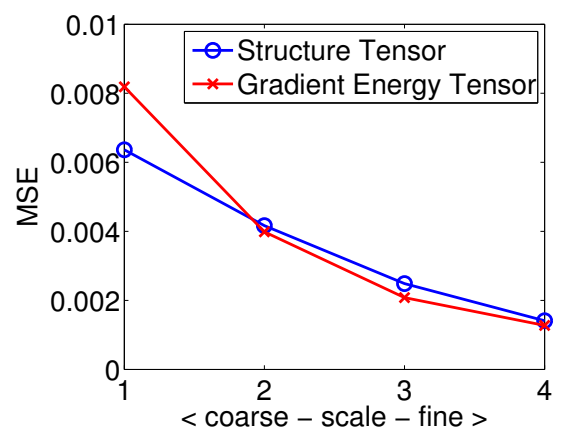

Dumptruck

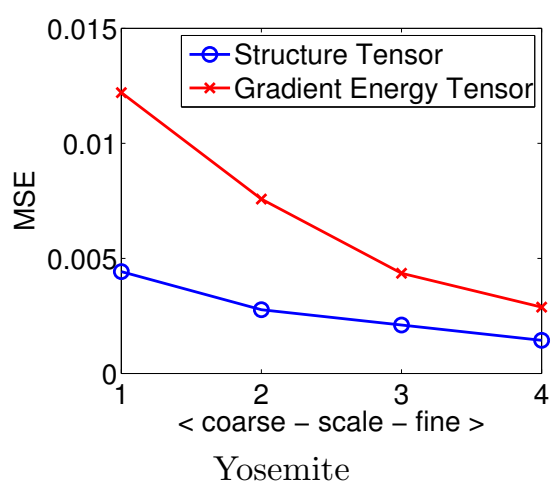

Figure 8.5: Mean squared error (MSE) between the first and the second image of sequences from the Middlebury dataset [13. The estimate of the motion field in the Wooden sequence for the gradient energy tensor diverges at the finest scale, but in the other sequences it yields a comparable displacement estimate with regard to MSE.

when we replace the structure tensor with the gradient energy tensor with positive eigenvalues, i.e., $G E T^{+}$in 8.7. Due to the large displacement between the image pairs we are required to have a post-convolution of the $G E T^{+}$components similarly to the structure tensor in order to capture the motion.

The normal equation 8.11) is solved explicitly for both tensors using the pseudo-inverse at multiple scales. The solution at a coarse scale is propagated to a finer scale and after each scale we apply a median-filter to the estimated motion field [86]. Furthermore, it was found that the magnitude scaling of the gradient energy tensor eigenvalues resulted in a poor motion estimation. Therefore, the eigenvalues of the gradient energy tensor were scaled using the factor $\sigma i / I$ where $i \in I=\{1,2,3,4\}$ are the scales, $i=1$ is the fines scale, and $\sigma$ is the standard deviation of the Gaussian filter $w(x)$. As for the structure tensor, the selection of $\sigma$ depends on the absolute motion present within the frames, i.e., if the displacement is large then a larger $\sigma$ is required. 

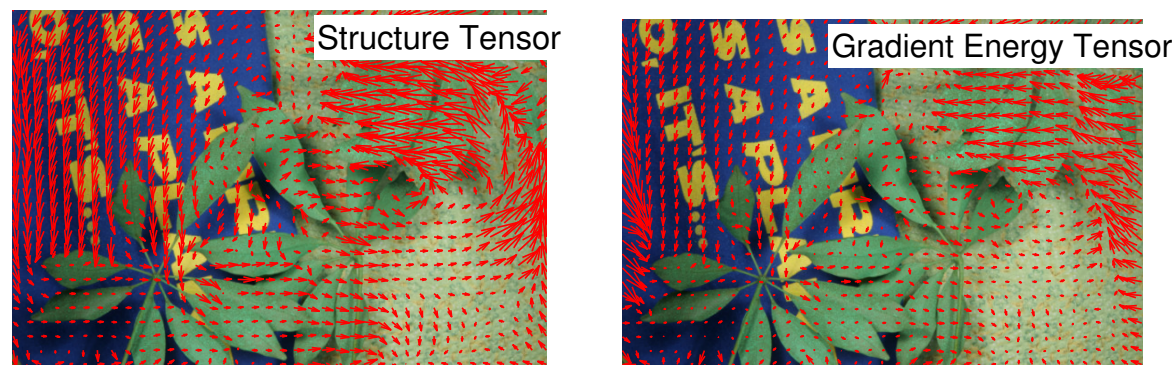

Structure Tensor

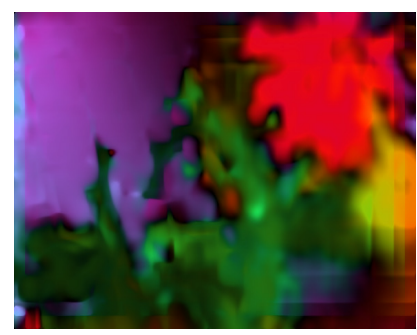

Gradient Energy Tensor
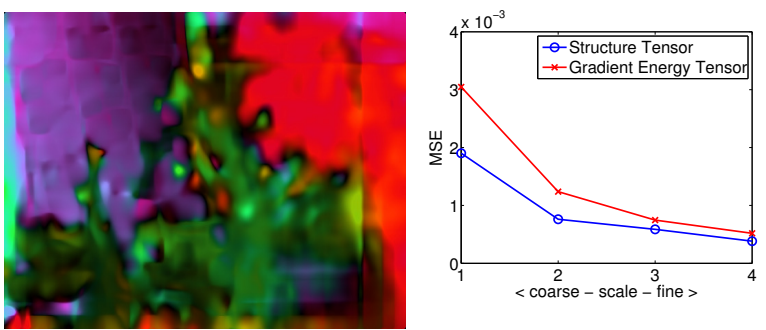

Figure 8.6: Optical flow estimated from frames 7 and 8 of the Schefflera sequence in the Middlebury optical flow dataset [13. Top row illustrate the vector field. On the bottom we show the obtained flow fields where the direction is colour coded and intensity corresponds to the magnitude. The graph to the right show the mean squared error between the warped image $J(x+d)$ and the reference image $I(x)$ in 8.10).

Figure 8.5 shows the mean squared error between $J(x+d)$ and $I(x)$ for the sequences Backyard, Dumptruck, Wooden and Yosemite from the Middlebury optical flow dataset [13. In each of the examples the difference of the mean squared error for the two tensors is large at the coarsest scale. Nevertheless, at the finest scale, the estimated displacement fields show similar errors values for each sequence. The Wooden sequence is a failure case for the gradient energy tensor since the displacement field has a worse estimate on the finest scale compared to the previous scale. The reason is that the scaling factor $\sigma_{i} / I$ of the gradient energy tensor eigenvalues is not generic enough to capture the motion in this example.

The optical flow formulation 8.10 clearly does not give state-of-the-art results, however the approach illustrates that the gradient energy tensor is a possible alternative to the structure tensor. One of the primary reasons for the inferior motion estimate, is that the tensor fails to describe the image signal in a large enough neighbourhood. Therefore we were forced to add a spatial post-convolution of the tensor-components. Furthermore it is possible to use a more fine grained scale pyramid than was used in this work, but this would increase the computation time. Nevertheless, we expect that many interesting further results can be derived from this approach. 


\section{Chapter 9}

\section{Gradient energy total variation}

This chapter introduces the gradient energy tensor-based total variation (GETV) scheme. The material is adapted from our work [3].

The total variation (TV) formulation by Rudin et al. [79] is defined as the regularization term

$$
R(u)=\int_{\Omega}|\nabla u| d x .
$$

The TV-model is well studied, both theoretically and practically, for an overview of the method and its application areas see Chan et al. 24]. One major drawback with the TV-approach, for image denoising applications, is its tendency to enforce piecewise-constant surfaces. The problem can be reduced by introducing additional smoothness constraints, e.g., as done by Bredies et al. 20].

The main goal of this chapter is to extend the total variation framework with an operator that captures gradient magnitude changes, both in direction and size, i.e., the aim is to exploit a tensor-valued formulation of total variation which does not suffer from piecewise-constant surfaces in its solution.

Often the structure tensor is used for orientation estimates, however the tensor does not admit standard variational tools to compute the Euler-Lagrange equation. Therefore, this study proposes to replace the structure tensor with an alternative tensor, the gradient energy tensor from chapter 8. The approach allows for a formal derivation of the Euler-Lagrange equation that results in a tensor-based gradient energy total variation scheme.

The results are derived from Theorem 2. Additionally, a direct application of the gradient energy tensor is to exploit the tensor in Theorem 2. This means to solve 4.16 with $W(\nabla m(u))$ defined as

$$
W(\nabla m(u))=\exp \left(-G E T^{+}(\nabla m(u)) / k^{2}\right),
$$

where $G E T^{+}$is the gradient energy tensor with positive eigenvalues defined in (8.7).

The following sections first reviews existing tensor-based formulations of the total-variation framework before introducing the objective function which defines 
the gradient energy total variation (GETV) scheme.

\subsection{Tensor-based extensions of total variation}

The extension of TV to variational tensor-based formulations was investigated by Roussous and Maragos [78, Lefkimmiatis et al. 61] and Grasmair and Lenzen [4]. All works consider the structure tensor and model the objective function in terms of the tensor eigenvalues. Roussos and Maragos [78] define their regularization term as

$$
R(u)=\int_{\Omega} \psi\left(\mu_{1}, \mu_{2}\right) d x
$$

where $\mu_{1,2}$ are the eigenvalues of the structure tensor and $\psi$ is a convex function in both of its arguments. The regularization is only indirectly taking into account the structure tensor and ignores the eigenvectors. Lefkimmiatis et al. 61] similar to Roussous and Maragos, considered the Schatten-norm of the structure tensor eigenvalues. In contrast, Grasmair and Lenzen 44] defined the regularization term

$$
R(u)=\int_{\Omega} \sqrt{\nabla^{t} u D(u) \nabla u} d x,
$$

where $D(u)$ is the structure tensor with remapped eigenvalues. The objective function is minimized using a finite element method instead of deriving a variational solution. An alternative approach was proposed by Krajsek and Scharr [59] who formulated a linear anisotropic regularization term resulting in an approximate formulation of a tensor-valued functional. The common formulation by the aforementioned works is that they use the structure tensor. Due to the fact that the structure tensor is defined as the integration of the outer product of the image gradient, the divergence theorem and the variational framework are not applicable when deriving a formal minimizer of the functional.

\subsection{Definition of gradient energy total variation}

This section derives the gradient energy total variation scheme. The derivation is based on a series of eigenvalue decompositions before arriving at the desired result. Additionally, we will study properties and interpretation of the GETV before showing the corresponding Euler-Lagrange equation in the next section.

The regularization term we consider is given in the following definition.

Definition 2 (Gradient energy total variation). The gradient energy total variation functional (GETV) is

$$
R(u)=\int_{\Omega} \nabla^{t} u S(\nabla u) \nabla u d x,
$$

where $S(\nabla u) \in \mathbb{R}^{2 \times 2}$ is a symmetric positive semi-definite tensor.

We begin our analysis by putting $S(\nabla u) \in \mathbb{R}^{2 \times 2}$ to be the symmetric positive semi-definite tensor in 9.5 with eigenvalues $\mu_{1,2}$ and orthonormal eigenvectors 
$v, w$ such that

$$
S(\nabla u)=\mu_{1} v v^{t}+\mu_{2} w w^{t}
$$

Furthermore, define a tensor $W(\nabla u) \in \mathbb{R}^{2 \times 2}$ which also is symmetric positive semi-definite with corresponding eigenvectors to $S(\nabla u)$, i.e.

$$
W(\nabla u)=\lambda_{1} v v^{t}+\lambda_{2} w w^{t}
$$

and $\lambda_{1,2}$ are the eigenvalues.

In particular, consider $S(\nabla u)$ of the form

$$
W(\nabla u)=|\nabla u| S(\nabla u)
$$

such that 9.5 is convex (see Corollary 2 . Then, from 4.35 we have (restated for clarity)

$$
\nabla^{t} u W(\nabla u) \nabla u=\lambda_{1}|\nabla u|^{2}(v \cdot p)^{2}+\lambda_{2}|\nabla u|^{2}(w \cdot p)^{2}
$$

Now, note that by defining $W(\nabla u)$ as in (9.8), we can reduce the exponent of $|\nabla u|^{2}$ in $(9.9$ with one degree, and thus we do not have a singular point. Hence, by the definition of $S(\nabla u)$ as in $(9.8)$ we obtain

$$
\nabla^{t} u S(\nabla u) \nabla u=\lambda_{1}|\nabla u|(v \cdot p)^{2}+\lambda_{2}|\nabla u|(w \cdot p)^{2}
$$

i.e., a tensor-based total variation formulation.

We set $W(\nabla u)$ in $(9.8)$ as 9.2 where $G E T^{+}$is a positive semi-definite tensor

$$
\operatorname{GET}^{+}(\nabla u)=U\left(\begin{array}{cc}
\left|\iota_{1}\right| & 0 \\
0 & \left|\iota_{2}\right|
\end{array}\right) U^{t} .
$$

Here, $U$ denote the eigenvectors and $\iota_{1,2}$ the eigenvalues of $G E T$. Also, let exp be the matrix exponential function of $G E T^{+}$such that $\lambda_{i}=\exp \left(-\left|\iota_{i}\right| / k\right)$ for $i=1,2$ and $k>0$.

Remark 3 (GETV reduction to TV). The standard total variation formulation is obtained from (9.8) by setting $W(\nabla u)$ as the identity matrix I, then we have

$$
\Phi(\nabla u)=\nabla^{t} u \frac{I}{|\nabla u|} \nabla u=\frac{|\nabla u|^{2}}{|\nabla u|}=|\nabla u| .
$$

Notice that we can derive the same result from (4.40). Suppose that $W(\nabla u)=I$, then $\lambda_{1,2}=1$ and $\sigma_{1}(A)=|\nabla u|$ and $\sigma_{2}(A)=0$, which gives that

$$
\Phi(\nabla u)=\|A(\nabla u)\|_{1}=|\nabla u| .
$$




\subsubsection{Formal minimizer}

The previous section defined the GETV in Definition 2 by putting $S(\nabla u)$ according to 9.8 with $W(\nabla u)$ as in 9.2 with $m(u)=u$. In order to minimize the proposed functional we use Theorem 2, which states the corresponding EulerLagrange equation for a functional with a quadratic form. Therefore we use this result to directly minimize 4.15 with $m(u)=u$. By using Theorem 2 we compute $S_{\nabla u}$ as

$$
S_{\nabla u}=-\frac{1}{|\nabla u|^{3}}\left(\begin{array}{c}
u_{x} \nabla^{t} u W \\
u_{y} \nabla^{t} u W
\end{array}\right)+\frac{1}{|\nabla u|}\left(\begin{array}{c}
\nabla^{t} u W_{u_{x}} \\
\nabla^{t} u W_{u_{y}}
\end{array}\right),
$$

so that the corresponding minimizer of the regularizer 4.15 is obtained by inserting 9.14 into 4.31

$$
\left\{\begin{array}{rl}
u-u_{0}-\lambda \operatorname{div}\left(Q \frac{\nabla u}{|\nabla u|}\right) & =0 \text { in } \Omega \\
n \cdot\left(S_{u_{x}}+S_{u_{y}}\right) \nabla u & =0 \text { on } \partial \Omega
\end{array},\right.
$$

where

$$
Q=2 W+\left(\begin{array}{l}
\nabla^{t} u W_{u_{x}} \\
\nabla^{t} u W_{u_{y}}
\end{array}\right)-\frac{1}{|\nabla u|^{2}}\left(\begin{array}{l}
u_{x} \nabla^{t} u W \\
u_{y} \nabla^{t} u W
\end{array}\right),
$$

is a weight controlling the anisotropy of the total variation scheme. We compute the component-wise derivative of $W$ with respect to $u_{x}$ and $u_{y}$ by using an explicit eigendecomposition, i.e.

$$
\begin{aligned}
W_{u_{x}}= & {\left[\left(\begin{array}{cc}
2 v_{1} & v_{2} \\
v_{2} & 0
\end{array}\right)\left(\partial_{u_{x}} v_{1}\right)+\left(\begin{array}{cc}
0 & v_{1} \\
v_{1} & 2 v_{2}
\end{array}\right)\left(\partial_{u_{x}} v_{2}\right)\right] \lambda_{1}+v v^{t}\left(\partial_{u_{x}} \lambda_{1}\right) } \\
& +\left[\left(\begin{array}{cc}
2 w_{1} & w_{2} \\
w_{2} & 0
\end{array}\right)\left(\partial_{u_{x}} w_{1}\right)+\left(\begin{array}{cc}
0 & w_{1} \\
w_{1} & 2 w_{2}
\end{array}\right)\left(\partial_{u_{x}} w_{2}\right)\right] \lambda_{2}+w w^{t}\left(\partial_{u_{x}} \lambda_{2}\right),
\end{aligned}
$$

with the corresponding orthonormal eigenvectors $v$ and $w$. The generalised expressions for the derivatives of the eigenvalues and eigenvectors are given in appendix A.3.

The most intuitive interpretation of the GETV is to consider the eigendecomposition of $W(\nabla u)$. Thus given eigenvalues $\lambda_{1,2}=\exp \left(-\left|\iota_{1,2}\right| / k\right)$ of $W(\nabla u)$, the exponential function will adapt the filtering to be parallel to the image structures, i.e., close to an image structure $\lambda_{1}$ will be small and $\lambda_{2}$ larger. Since the gradient energy tensor does not contain a post-convolution of the tensor-components, our formulation allows us to better preserve fine details in the image structure, than if we would use the structure tensor, as we show in the numerical experiments in section 13.2 


\subsection{Generalised formulation}

In this section we give the general formulation of GETV that includes the mapping function, GETVm. The regularization term we consider is given in the following definition.

Definition 3 (GETV generalised formulation). The gradient energy total variation functional with a mapping function (GETVm) is

$$
R(u)=\int_{\Omega} \nabla^{t} m(u) S(\nabla m(u)) \nabla m(u) d x,
$$

where $S(\nabla m(u)) \in \mathbb{R}^{2 \times 2}$ is a symmetric positive semi-definite tensor.

Let $R(u)$ be the regularization term expressed on the form 9.18 with

$$
W(\nabla m(u))=S(\nabla m(u))|\nabla m(u)|
$$

Then the resulting tensor $S_{\boldsymbol{s}}$ is

$$
S_{\boldsymbol{s}}=-\frac{1}{|\nabla s|^{3}}\left(\begin{array}{c}
s_{1} \nabla^{t} u W \\
s_{2} \nabla^{t} u W
\end{array}\right)+\frac{1}{|\nabla s|}\left(\begin{array}{c}
\nabla^{t} u W_{s_{1}} \\
\nabla^{t} u W_{s_{2}}
\end{array}\right),
$$

where

$$
W=\exp \left(-G E T^{+}(\nabla m(u)) / k^{2}\right) .
$$

The explicit computation of the E-L equation is extensive and is given in appendix A.3. 


\section{Chapter 10}

\section{Numerical schemes}

This chapter considers the numerical aspects of implementing the presented diffusion methods. Moreover, the chapter presents the derivation of finite difference operators and gives an example implementation in Matlab-code that describes the forward Euler-scheme using convolution masks.

\section{$10.1 \quad$ Numerical implementation}

A diffusion process, as the ones described in this thesis, can be solved numerically by considering an evolution equation of the form

$$
\partial_{t} u=A(u) u
$$

where $A$ is a spatially dependent linear operator containing, e.g., the derivative operators on its diagonals. An iterative discretisation scheme can be written as

$$
\frac{u^{i+1}-u^{i}}{\lambda}=\beta A\left(u^{i}\right) u^{i+1}+(1-\beta) A\left(u^{i}\right) u^{i},
$$

where $0 \leq i<\infty$ is the current iteration. If $\beta=1$ then 10.2 denotes a fully implicit scheme, if $\beta=1 / 2$ then the scheme is semi-implicit and if $\beta=0$ a fully explicit scheme is obtained. If $\beta>0$ then it is necessary to solve an equation system of size $N^{2} \times N^{2}$ in each iteration, where $N$ is the number of pixels in the image. Throughout this work the explicit scheme has been used due to its simplicity. The explicit scheme reads

$$
u^{i+1}=u^{i}+\lambda A\left(u^{i}\right) u^{i}
$$

and the matrix $A$, also known as the stencil, can be expressed with a sparse representation making memory requirements less demanding. 


\begin{tabular}{|c|c|c|}
\hline 0 & + & 0 \\
\hline+ & + & + \\
\hline 0 & + & 0 \\
\hline
\end{tabular}

(a)

\begin{tabular}{|c|c|c|}
\hline$?$ & + & $?$ \\
\hline+ & + & + \\
\hline$?$ & + & $?$ \\
\hline
\end{tabular}

(b)

Figure 10.1: Stencil signs when $D$ is (a) a scalar or (b) a tensor 97.

A straightforward discretisation of the divergence forms that have been introduced in previous chapters is to do the following expansion

$$
\begin{aligned}
\operatorname{div}(D \nabla u) & =\operatorname{div}\left(\begin{array}{l}
d_{11} \partial_{x} u+d_{12} \partial_{y} u \\
d_{21} \partial_{x} u+d_{22} \partial_{y} u
\end{array}\right) \\
& =\partial_{x}\left(d_{11} \partial_{x} u\right)+\partial_{x}\left(d_{12} \partial_{y} u\right)+\partial_{y}\left(d_{21} \partial_{x} u\right)+\partial_{y}\left(d_{22} \partial_{y} u\right) .
\end{aligned}
$$

where $D$ is a tensor and the subindex of $d$ its corresponding components by row and column.

From 10.4 it is seen that both first and the second order derivatives need to be approximated. A common method is to use finite differences, introduced in the next section. An important note is that when approximating (10.4) one can attain negative values in the stencil due to the mixed derivatives components $\partial_{x} \partial_{y}$.

The signs of the stencil, obtained for $D$ being a scalar function, as in (3.13) or a tensor as in (3.18) are illustrated in figure 10.1. The possible existence of negative factors in the stencil, indicated by ?, can lead to over- and undershoots at the image structure borders. However if the step length, $\lambda$, is small enough then these artefacts are rarely observed. Non-negative schemes have been constructed and are proven to yield positive entries in the stencils. For example, the non-negative scheme by Weickert [95] was used in section 13.1.

\section{$10.2 \quad$ Finite difference operators}

An important aspect of solving the PDEs introduced in this thesis is how to discretize derivatives. Our approach, based on finite difference operators, consists of three operators; the forward operator $\partial^{+}$, the backward operator $\partial^{-}$, and the central difference operator $\partial^{c}=\left(\partial^{+}+\partial^{-}\right) / 2$.

Let $h$ be the size of one pixel given in $x$ - and $y$-direction. Then a third order two-dimensional Taylor series expansion in the $x$-direction and $y$-direction, respectively, reads

$$
\begin{aligned}
& u(x+h, y)=u(x, y)+h u_{x}(x, y)+\frac{h^{2}}{2} u_{x x}(x, y)+O\left(h^{3}\right), \\
& u(x-h, y)=u(x, y)-h u_{x}(x, y)+\frac{h^{2}}{2} u_{x x}(x, y)+O\left(h^{3}\right), \\
& u(x, y+h)=u(x, y)+h u_{y}(x, y)+\frac{h^{2}}{2} u_{y y}(x, y)+O\left(h^{3}\right), \\
& u(x, y-h)=u(x, y)-h u_{y}(x, y)+\frac{h^{2}}{2} u_{y y}(x, y)+O\left(h^{3}\right) .
\end{aligned}
$$


The Taylor expansions in the diagonal directions are

$$
\begin{aligned}
u(x+h, y+h)=u+ & h u_{x}+h u_{y} \\
& +\frac{h^{2}}{2}\left(u_{x x}+2 u_{x y}+u_{y y}\right)+O\left(h^{3}\right), \\
u(x+h, y-h)=u+ & h u_{x}-h u_{y} \\
& +\frac{h^{2}}{2}\left(u_{x x}-2 u_{x y}+u_{y y}\right)+O\left(h^{3}\right), \\
u(x-h, y+h)=u- & h u_{x}+h u_{y} \\
& +\frac{h^{2}}{2}\left(u_{x x}-2 u_{x y}+u_{y y}\right)+O\left(h^{3}\right), \\
u(x-h, y-h)=u- & h u_{x}-h u_{y} \\
& +\frac{h^{2}}{2}\left(u_{x x}+2 u_{x y}+u_{y y}\right)+O\left(h^{3}\right),
\end{aligned}
$$

where the argument $(x, y)$ has been dropped for increased clarity. From 10.5 and 10.6 it is possible to derive the forward and backward finite difference operators $\partial^{+}$and $\partial^{-}$as second order approximations in the $x$-direction

$$
\begin{aligned}
& \partial_{x}^{+} u=\frac{u(x+h, y)-u(x, y)}{h}+O\left(h^{2}\right), \\
& \partial_{x}^{-} u=\frac{u(x, y)-u(x-h, y)}{h}+O\left(h^{2}\right), \\
& \partial_{x}^{c} u=\frac{u(x+h, y)-u(x-h, y)}{2 h}+O\left(h^{3}\right),
\end{aligned}
$$

and by using 10.7 and 10.8 the corresponding operators in the $y$-direction are obtained.

With the central differences, the second order derivatives are computed by summing 10.5 and 10.6, respectively 10.7 and 10.8

$$
\begin{aligned}
& u_{x x}(x, y)=\frac{u(x+h, y)-2 u(x, y)+u(x-h, y)}{h^{2}}+O\left(h^{3}\right), \\
& u_{y y}(x, y)=\frac{u(x, y+h)-2 u(x, y)+u(x, y-h)}{h^{2}}+O\left(h^{3}\right),
\end{aligned}
$$

and the mixed-derivative filter is obtained by summing 10.9 and 10.12 and subtracting 10.10 and 10.11 which result in the third-order approximation

$$
\begin{aligned}
u_{x y}(x, y) \approx \frac{1}{4 h^{2}} & (u(x+h, y+h)-u(x+h, y-h) \\
& -u(x-h, y+h)+u(x-h, y-h)) .
\end{aligned}
$$

In some cases it is useful to write the finite difference operators as convolution masks. Figure 10.2 illustrates the convolution masks derived in this section as well as the first order derivative of the $y$-component. 


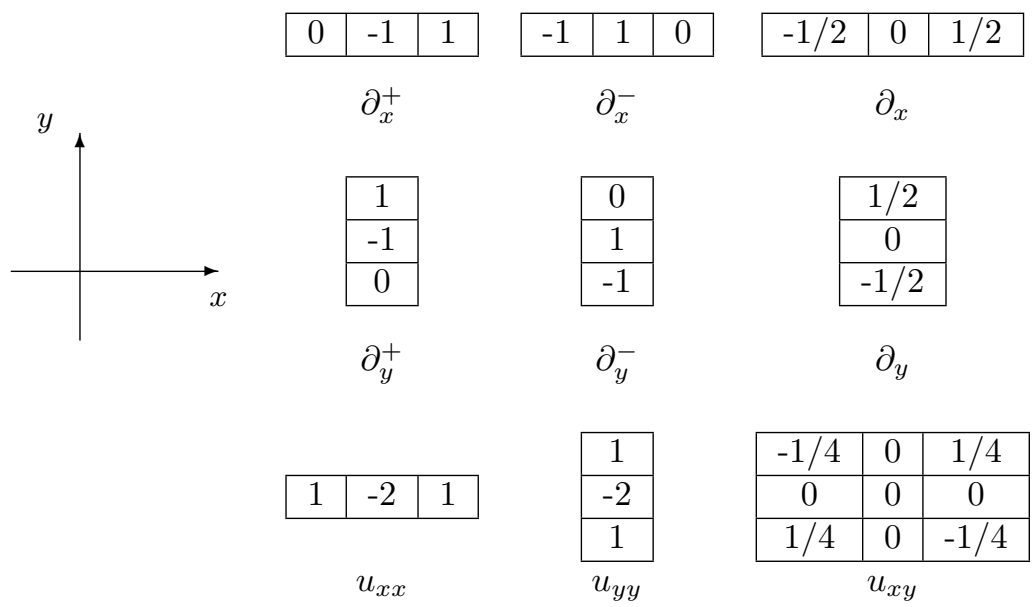

Figure 10.2: Convolution masks, where $h=1$ and the coordinate system on the left indicates the respective $x$ and $y$-orientation.

\subsection{Approximation of the divergence operator}

Let the image $u$ be sampled on a grid where the spacing between two points on the grid has the distance $h=1$. This section describes in detail how to discretize 10.4 and it is an adaptation of the work 97.

To get a symmetric scheme it is common to discretize mixed derivatives using central differences

$$
\begin{aligned}
& \partial_{x}^{c} u=\frac{1}{2}(u(x+1, y)-u(x-1, y)), \\
& \partial_{y}^{c} u=\frac{1}{2}(u(x, y+1)-u(x, y-1)),
\end{aligned}
$$

and the squared ones using forward and backward differences

$$
\begin{array}{ll}
\partial_{x}^{+} u=u(x+1, y)-u(x, y) & \partial_{y}^{+} u=u(x, y+1)-u(x, y), \\
\partial_{x}^{-} u=u(x, y)-u(x-1, y) & \partial_{y}^{-} u=u(x, y)-u(x, y-1),
\end{array}
$$

finite difference elements. Below, we derive the expressions which discretize 10.4).

\subsubsection{Second $x$-derivative (horizontal)}

Here, we use an alternating scheme of forward and backward differences for the $x$-directions of the divergence components. Let

$$
\partial_{x}\left(d_{11} \partial_{x} u\right) \approx \frac{1}{2}\left(\partial_{x}^{+}\left(d_{11} \partial_{x}^{-} u\right)+\partial_{x}^{-}\left(d_{11} \partial_{x}^{+} u\right)\right)
$$


be the approximation, then

$$
\begin{array}{r}
\partial_{x}^{+}\left(d_{11} \partial_{x}^{-} u\right)=\partial_{x}^{+}\left(d_{11}(x, y)(u(x, y)-u(x-1, y))\right) \\
=d_{11}(x+1, y)(u(x+1, y)-u(x, y)) \\
\quad-d_{11}(x, y)(u(x, y)-u(x-1, y)),
\end{array}
$$

and

$$
\begin{aligned}
\partial_{x}^{-}\left(d_{11} \partial_{x}^{+} u\right)= & \partial_{x}^{-}\left(d_{11}(x, y)(u(x+1, y)-u(x, y))\right) \\
= & d_{11}(x, y)(u(x+1, y)-u(x, y)) \\
& \quad-d_{11}(x-1, y)(u(x, y)-u(x-1, y)),
\end{aligned}
$$

so that the first component of 10.4 is approximated by

$$
\begin{aligned}
\partial_{x}\left(d_{11} \partial_{x} u\right) & \approx \frac{1}{2}\left[\left(d_{11}(x+1, y)+d_{11}(x, y)\right)(u(x+1, y)-u(x, y))\right. \\
& \left.-\left(d_{11}(x-1, y)+d_{11}(x, y)\right)(u(x, y)-u(x-1, y))\right] .
\end{aligned}
$$

\subsubsection{Second $y$-derivative (vertical)}

The component with two differentiations in the $y$-direction is approximated as

$$
\partial_{y}\left(d_{22} \partial_{y} u\right) \approx \frac{1}{2}\left(\partial_{y}^{+}\left(d_{22} \partial_{y}^{-} u\right)+\partial_{y}^{-}\left(d_{22} \partial_{y}^{+} u\right)\right)
$$

then

$$
\begin{array}{r}
\partial_{y}^{+}\left(d_{22} \partial_{y}^{-} u\right)=\partial_{y}^{+}\left(d_{22}(x, y)(u(x, y)-u(x, y-1))\right) \\
=d_{22}(x, y+1)(u(x, y+1)-u(x, y)) \\
\quad-d_{22}(x, y)(u(x, y)-u(x, y-1)),
\end{array}
$$

and

$$
\begin{aligned}
\partial_{y}^{-}\left(d_{22} \partial_{y}^{+} u\right)= & \partial_{y}^{-}\left(d_{22}(x, y)(u(x, y+1)-u(x, y))\right) \\
= & d_{22}(x, y)(u(x, y+1)-u(x, y)) \\
& \quad-d_{22}(x, y-1)(u(x, y)-u(x, y-1)) .
\end{aligned}
$$

Taking the sum of the two previous expansions yield

$$
\begin{aligned}
\partial_{y}\left(d_{22} \partial_{y} u\right) & \approx \frac{1}{2}\left[\left(d_{22}(x, y+1)+d_{22}(x, y)\right)(u(x, y+1)-u(x, y))\right. \\
& \left.-\left(d_{22}(x, y-1)+d_{22}(x, y)\right)(u(x, y)-u(x, y-1))\right] .
\end{aligned}
$$




\subsubsection{Mixed derivative (diagonal)}

The approximation of the $x y$-mixed derivative for $d_{12}$ is computed with central differences, i.e.

$$
\partial_{x}\left(d_{12} \partial_{y} u\right) \approx \partial_{x}^{c}\left(d_{12} \partial_{y}^{c} u\right)
$$

and

$$
\begin{aligned}
\partial_{x}^{c}\left(d_{12} \partial_{y}^{c} u\right) & =\partial_{x}^{c}\left(d_{12}(x, y) \frac{1}{2}(u(x, y+1)-u(x, y-1))\right) \\
& =\frac{1}{4}\left[\left(d_{12}(x+1, y)(u(x+1, y+1)-u(x+1, y-1))\right.\right. \\
& \left.-d_{12}(x-1, y)(u(x-1, y+1)-u(x-1, y-1))\right] .
\end{aligned}
$$

Analogously, the $y x$-derivative for the $d_{21}$ component reads

$$
\partial_{y}\left(d_{21} \partial_{x} u\right) \approx \partial_{y}^{c}\left(d_{21} \partial_{x}^{c} u\right)
$$

thus

$$
\begin{aligned}
\partial_{y}^{c}\left(d_{21} \partial_{x}^{c} u\right) & =\partial_{y}^{c}\left(d_{21}(x, y) \frac{1}{2}(u(x+1, y)-u(x-1, y))\right) \\
& =\frac{1}{4}\left[\left(d_{21}(x, y+1)(u(x+1, y+1)-u(x-1, y+1))\right.\right. \\
& \left.-d_{21}(x, y-1)(u(x+1, y-1)-u(x-1, y-1))\right] .
\end{aligned}
$$

\subsubsection{Example implementation}

This part describes the actual realization of the above numerical approximation that has been used throughout the experimental evaluation in subsequent chapters. The forward Euler-scheme, or explicit scheme, described in 10.3), using pseudocode, reads

$$
\mathrm{u}=\mathrm{u}+\text { stepsize } * \text { update_map }(\mathrm{u}, \mathrm{d} 11, \mathrm{~d} 12, \mathrm{~d} 21, \mathrm{~d} 22)
$$

where the d-index variables are components of a tensor and the stepsize variable denotes $\lambda$. The function update_map is listed in table 10.1 and determines the modification of the image values. Since the E-L equations that we consider are defined using Neumann boundary condition we need to define a zero-flow at the image borders, i.e., $n \cdot \nabla u=0$. The most straightforward way of achieving this is to extend the image border by 1 pixel using replication. This is done in the function conv2extend in table 10.1. which also applies a convolution filter. 


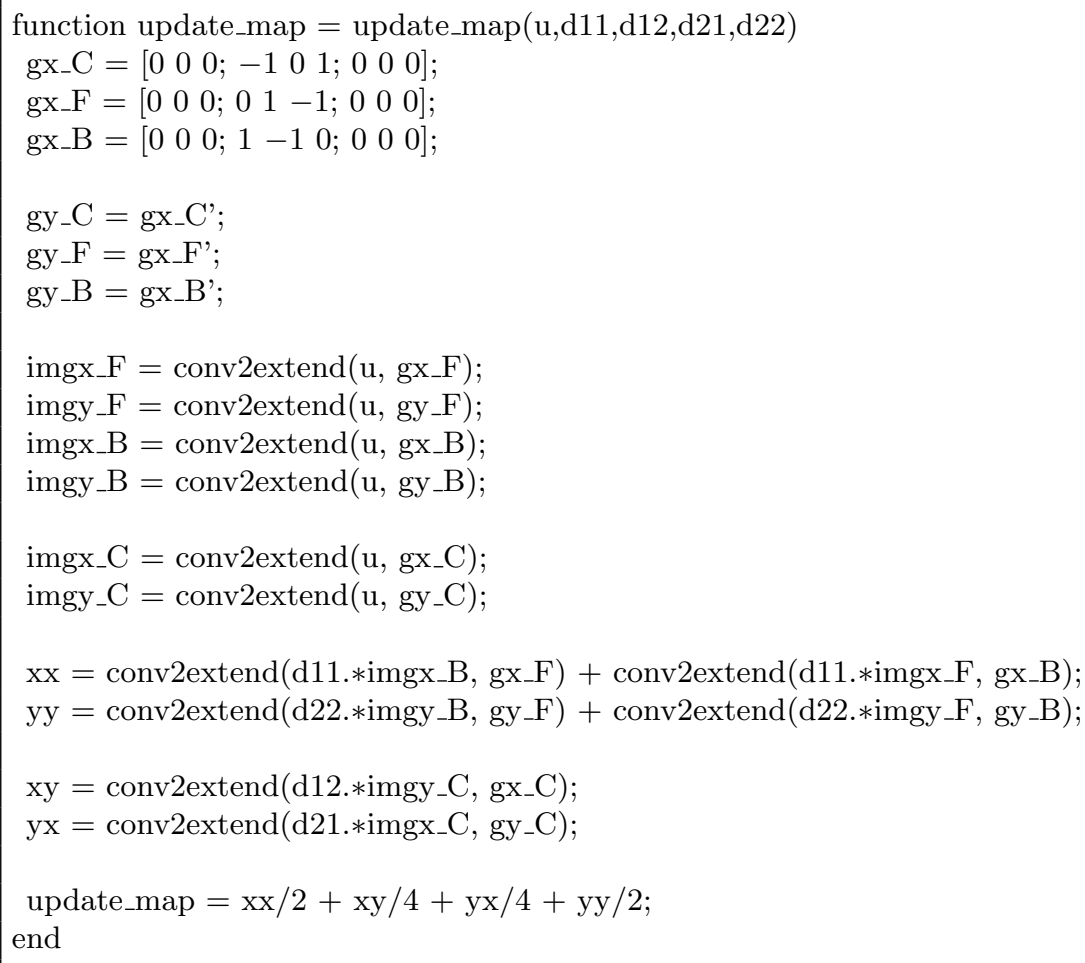

Table 10.1: Example of how an actual numerical implementation of the approximated divergence operator can be realized in Matlab code. The function conv2extend replicates the image border and applies a convolution kernel. This is a simple approach to obtain the Neumann boundary condition numerically, i.e., $n \cdot \nabla u=0$. 


\section{Chapter 11}

\section{Evaluation framework}

This chapter introduces the colour image representation and the evaluation criteria for the scalar-valued experiments in chapter 12 and the tensor-valued experiments in chapter 13. Before proceeding, note that the presented diffusion methods have not been limited by any particular image representation model such as spectral images, colour images, video sequences or greyscale images. Considering these different image modalities, particular image representations can be described as special cases of the more general theoretical results.

The extension from greyscale image processing to colour images still pose major problems due to the unknown mechanisms that govern colour perception. One of the most common colour spaces used to represent images is the RGB colour space, despite it being known that the colours (red, green and blue) are correlated due to physical properties such as the design of colour sensitive filters in camera equipment. Therefore, one natural question arises: what is a suitable colour space representation for image enhancement?

This thesis does not attempt to answer the question of the most suitable colour space representation for image enhancement. Instead we have settled for a transform constructed from a set of linear equations expressed in the RGB colour components. The resulting colour representation decorrelates the RGB colour space into an intensity component and two colour opponent components.

This chapter is adaptation from the author's licentiate thesis [1 and is restated here for the sake of completeness.

\subsection{Colour space representation}

Colour images are usually represented as components of the RGB (red, green and blue) colour space. The RGB colour space is sampled from this spectrum of visible light. Commonly, image data is represented using an 8 bit representation giving a total of 256 quantization levels. Since a colour image commonly contains 3 components one obtains a total of $3 \times 255$ values to represent the equiluminant 
colours from pure violet to pure red. If we are interested in one colour component and we vary its value from 0 to 255 , one obtains a transition from dark to light (i.e., from the colour black (value 0) to white (value 255)). Obviously, more quantization levels can represent a larger number of spectral components. Due to the structure of the light spectrum, colour components of the RGB colour space are highly correlated [82. The implication of this relation, for image filtering methods, is that by modifying one colour component, artefacts may be introduced at boundaries with sharp colour gradients. However, the most basic way of viewing an image is to model it as a greyscale image. To construct such a greyscale image from an RGB image, a simple approach is to average the colour components.

When vector-valued images are considered for image processing, primarily two approaches are possible. Either each component is considered separately and independently, or there is some (non-)linear combination of the colour components.

In [96] anisotropic diffusion in the RGB colour space is proposed, where the colour components are used to derive an averaged diffusion tensor. One drawback of this formulation is that colour artefacts can appear on the boundary of regions with sharp colour changes. Some alternative colour spaces investigated for image diffusion include manifolds [84, the CMY, HSV, Lab and Luv colour space [76], luminance and chormaticity [87, and our work 8 , that used the below colour transform for denoising in the opponent colour space.

The opponent colour transform that we considered in 8 is defined as 92,62 ,

$$
\begin{aligned}
O_{1} & =\frac{1}{\sqrt{3}}(R+G+B), \\
O_{2} & =\sqrt{\frac{2}{3}}\left(R-\frac{1}{2} G-\frac{1}{2} B\right), \\
O_{3} & =\frac{1}{\sqrt{2}}(G-B),
\end{aligned}
$$

where $O_{1}$ denotes the intensity and $O_{2}$ and $O_{3}$ are two components orthogonal to the intensity axis. The colour transformation is illustrated using a vector diagram in figure 11.1. In the left part of the figure, the RGB colour cube is depicted. The dark-grey hyperplane indicated by the vectors $\mathrm{R}, \mathrm{G}$ and $\mathrm{B}$ represents the case when the vector sum of the components is constant 1 , then the transform to the colour opponent plane is given on the right shown with the corresponding labels. The light-grey hyperplane composed of the vectors RB, RG and GB shows the case when the sum of the components is constant 2, the corresponding colour opponent transformation is also shown in the right figure. The interpretation of the colour transformation is that, e.g., the colour red is primarily described by a large contribution in $\mathrm{O}_{2}$, and that green and blue are opponent colours in the sign of $\mathrm{O}_{3}$.

The opponent transformation is derived from the assumption that permutations of the three RGB channels are on average equally probable 62. Using tools from the representation theory of the permutation group it can be shown that the result is a decorrelation of the original RGB variables into a one-dimensional intensity, $O_{1}$, and a two-dimensional colour-opponent component. In cases where the assumption of equally probable permutations is satisfied it can be shown that 


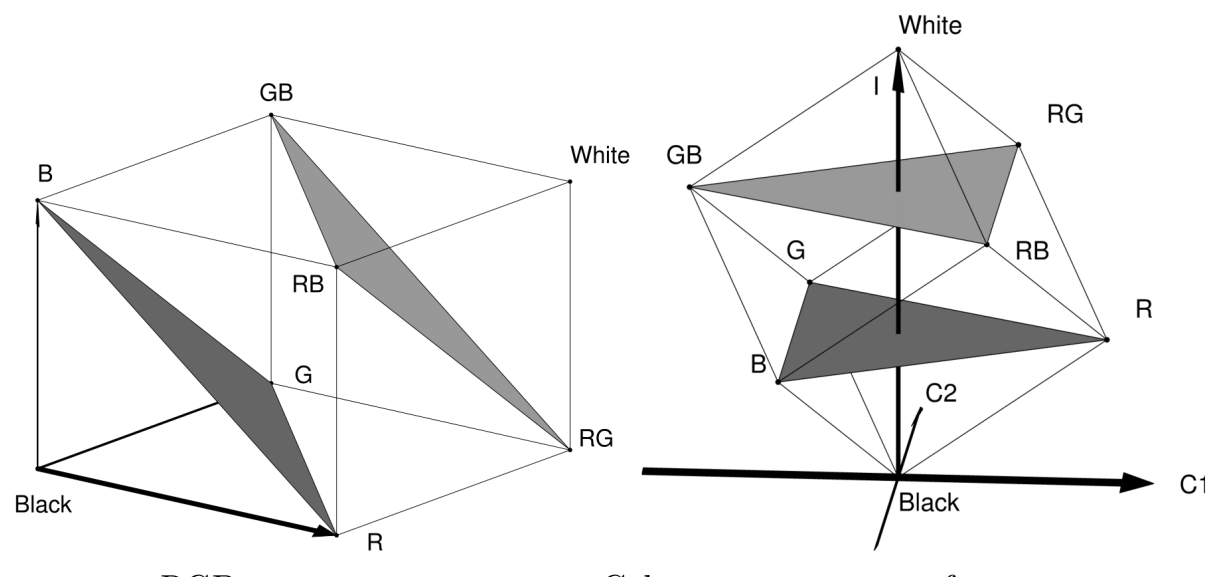

RGB

Colour opponent transform

Figure 11.1: Illustration of colour opponent transform. For the colour components $0 \leq R, G, B \leq 1$ the range of the opponent components are $0 \leq O_{1} \leq 3 / \sqrt{3}$, $-\sqrt{2 / 3} \leq O_{2} \leq \sqrt{2 / 3}$ and $-1 / \sqrt{2} \leq O_{3} \leq 1 / \sqrt{2}$.

the rows of this matrix are the eigenvectors of the correlation matrix computed from the RGB vectors and that the colour-opponent components belong to a twodimensional eigenspace belonging to the same eigenvalue.

This colour space representation was used in the experimental evaluation for density driven diffusion, D3, in section 12.3 and extended anisotropic diffusion in section 13.1. In particularly, the results in section 13.1, table 13.1 and figure 13.1 show the differences between filtering in the opponent colour space using tracebased (TR) diffusion (see (3.19) and [8, 33]) compared to the standard approach, i.e., averaging the tensor-components in the RGB colour space as introduced by Weickert 96. Comparing the peak signal-to-noise ratio (PSNR) and structural similarity (SSIM) values (introduced below) in table 13.1, it is obvious that filtering in the opponent colour space representation denoted by TR outperforms RGB, which denotes the standard approach, for every noise level and image. Additionally, considering the visual appearance of the final denoised images shown in figure 13.1, the standard approach clearly preserves noise in homogeneous regions, a drawback not visible when using the opponent space for these images and experimental setup.

\subsection{Image quality metrics}

Image quality is a subjective measure, and it is application dependent what is considered a good result. In this thesis, image quality has been assessed both qualitatively and quantitatively. Note that a qualitative assessment as "visually appealing" can be biased since it is a subjective measure and what appears as a good result for one person may not be generalised to others. Hence, the purpose of an image quality measurement is to not only model what is visually appealing, but 
also to model an objective benchmark measure that enables a comparison between two different competing methods.

\subsubsection{Mean squared error}

A quantitative measure that is commonly used to represent image quality is the mean squared error (MSE). It is computed by summing the squared difference between the noise-free image and the filtered image $u$, i.e.,

$$
\mathrm{MSE}=\frac{1}{N} \sum_{i=1}^{N}\left(\left(u_{0}\right)_{i}-u_{i}\right)^{2},
$$

where $N$ is the number of pixels in the image. Another popular measure, based on the MSE, is the peak signal-to-noise ratio (PSNR),

$$
\mathrm{PSNR}=10 \log _{10}\left(\frac{1}{M S E}\right),
$$

and $0 \leq u, u_{0} \leq 1$. However, as shown in 94 the perceptual quality does not correlate very well with the MSE and the PSNR metrics. With this motivation we often place larger importance on the structural similarity index (SSIM) as a measure for image quality, explained in the next section.

\subsubsection{Structural similarity index}

The Structural SIMmilarity index (SSIM) is an error measure which measures image similarity 94 . It is designed to be an objective image quality metric more consistent with how the human visual system perceives image quality. The fundamental difference between MSE and SSIM is that MSE is a metric which measures image quality in terms of absolute strength of the average error signal, whereas SSIM is a measure which compares local statistical properties of the signal thus giving an error estimate in terms of structural differences. The SSIM error value is computed as

$$
\operatorname{SSIM}(x, y)=\frac{\left(2 \mu_{x} \mu_{y}+C_{1}\right)\left(2 \sigma_{x y}+C_{2}\right)}{\left(\mu_{x}^{2}+\mu_{y}^{2}+C_{1}\right)\left(\sigma_{x}^{2}+\sigma_{y}^{2}+C_{2}\right)}
$$

In (11.6) $x$ and $y$ represent the two different images that are compared, one is the noisy image and the other one is the noise free image. The SSIM measure is designed based on the three components: luminance estimated by the mean value $\mu$, the signal contrast which is estimated from the standard deviation $\sigma$ and the structure similarity is computed according to the correlation coefficient between the images $x$ and $y . C_{1}$ and $C_{2}$ are two normalization constants.

In practice, the statistical properties are computed in local $8 \times 8$ pixel sized windows. However this may introduce block artefacts 94. Hence it is recommended that a small Gaussian filter is used as a weight function. Furthermore, rather than applying the SSIM index measure directly on the image, it is suggested 


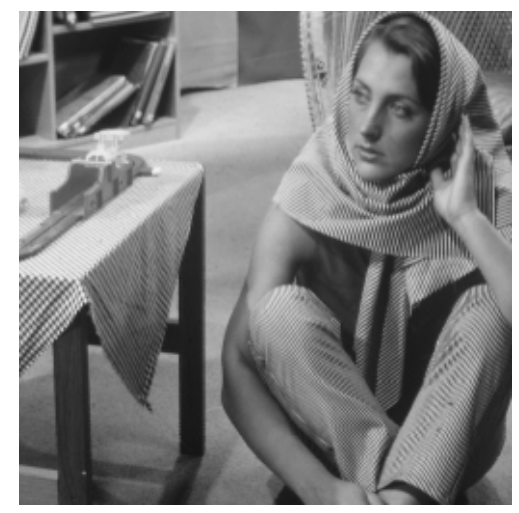

Barbara

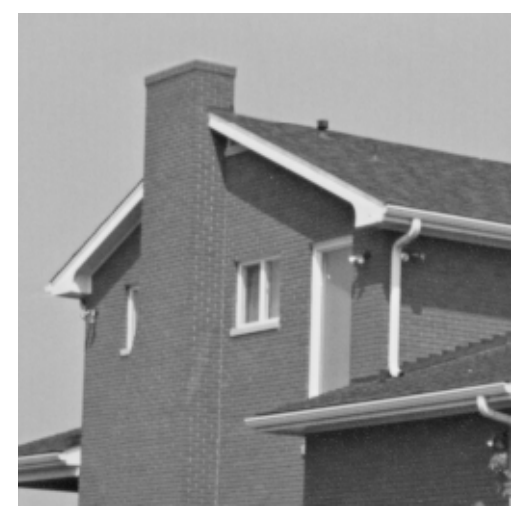

House

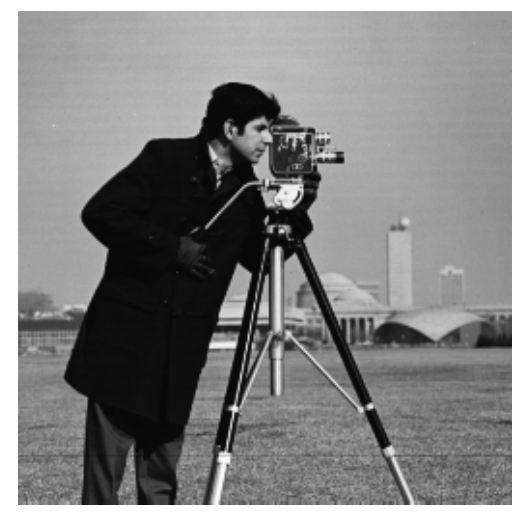

Cameraman

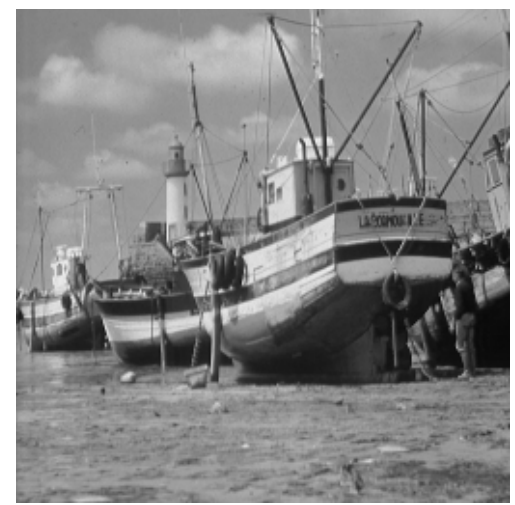

Boat

Figure 11.2: Standard greyscale test images used in the evaluation.

that the image scale is fitted to depend on view distance and image resolution. The authors of SSIM have made the implementation publicly available ${ }^{1}$. This is also the implementation of SSIM that is used in the subsequent evaluations.

\subsection{Image datasets}

In the image analysis community certain images are popular for algorithm benchmarks. This section gives a brief overview of the image datasets that have been used in the comparative studies. All images, expect the computed tomography (CT) dataset, are represented using an 8 bit quantization level for each (colour)channel.

\footnotetext{
1 www.cns.nyu.edu/ lcv/ssim/
} 


\section{Berkeley image segmentation dataset}

The Berkeley dataset 65] was originally created for the purpose of evaluating image segmentation and boundary detection algorithms. The dataset consists of 300 images of size $481 \times 321$ pixels, available both in RGB and greyscale versions.

\section{McGill calibrated colour image database}

The McGill database consists of about 850 images, each with resolution $786 \times 576$ pixels. 9 different categories of images are available and they include for example flowers, texture, animals, fruits and landscape scenes [71.

\section{Computed tomography dataset}

A computed tomography (CT) dataset was obtained through a cooperation with the CMIV, Center for Medical Imaging and Visualization, Linköping, Sweden. The database consists of $400 \mathrm{CT}$ images which were captured post-mortem. The scans have been acquired using a tube voltage of $120 \mathrm{kV}$, tube current of $127 \mathrm{mAs}$ and slice thickness of $1 \mathrm{~mm}$. The CT data is defined in Hounsfield units and is represented using a 12 bit quantization level.

\section{Commonly used test images}

Figure 11.2 depicts some famous greyscale images that are used for algorithmic evaluation and testing. This dataset includes the famous Cameraman, Lena and boat images among others.

\subsection{Evaluation setup}

This section presents the evaluation of methods that are related to Theorem 2. The subsequent chapters are divided into different themes shown in table 11.1. Each theme is coupled with a publication and the proposed method is marked by "o", the compared methods are marked by " $\mathrm{x}$ ". The aim has not been to compare the proposed algorithms to all possible denoising methods, rather we have primarily focused on methods related to the approach.

The PDEs are solved as initial value problems using a forward-Euler scheme and the image derivatives are approximated by using regularized finite differences as described in chapter 10 . The numerical approximation of the divergence operator is based on the expansion 10.4.

The image noise variance, $\sigma_{\text {est }}^{2}$, is estimated by using the technique in 38 . which is the foundation for the estimate of the contrast parameter in [33] (cf. (47) p. 6). The resulting diffusion parameter for the diffusion filters is then set as

$$
k=\frac{e-1}{e-2} \sigma_{e s t}^{2},
$$

where $e$ is the Euler number. A naive approach is taken to approximate the noise of the colour image, the noise estimate is the average of the estimated noise of 


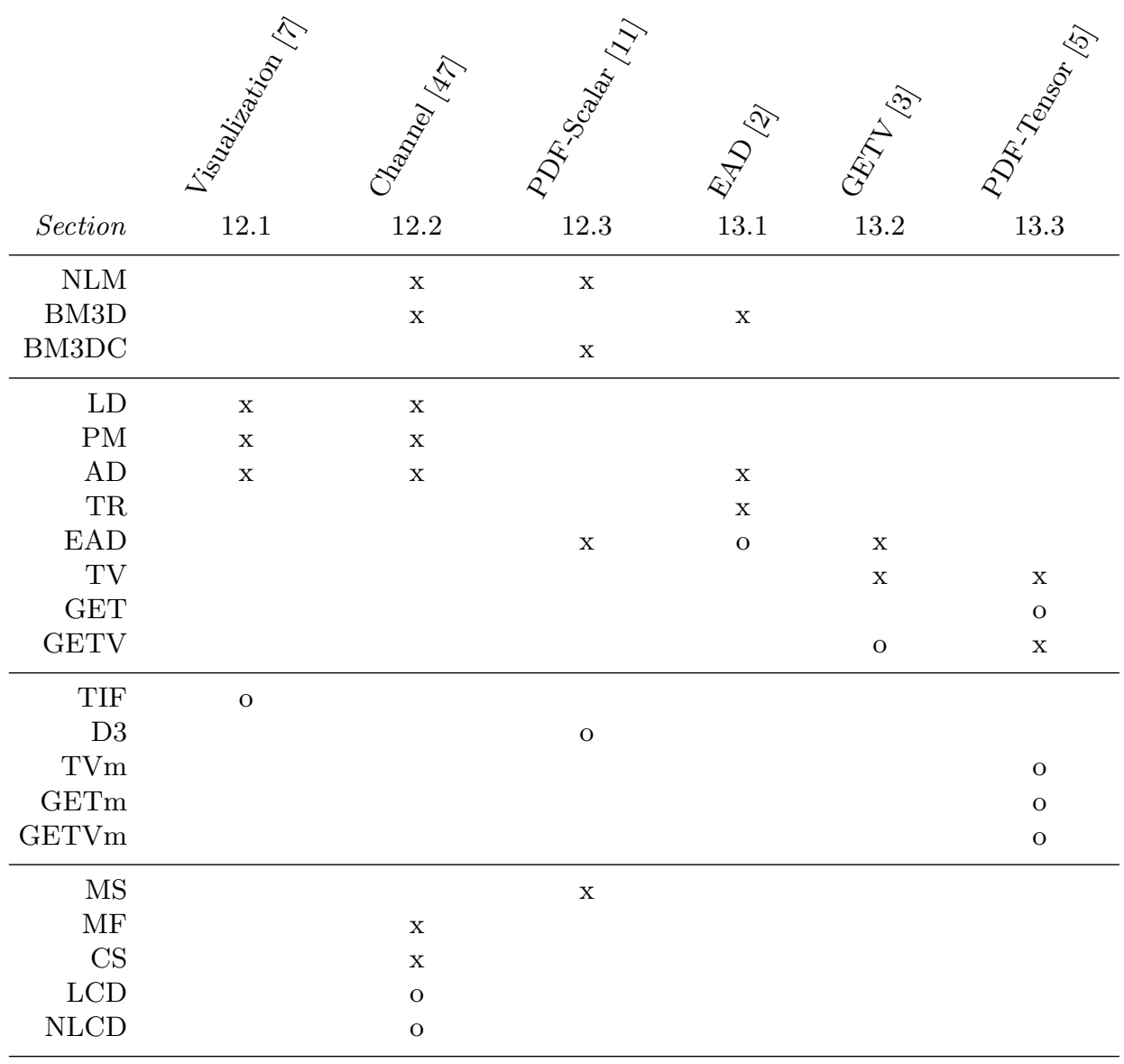

Table 11.1: Overview of the evaluated methods. PDF-Scalar and PDF-Tensor denote scalar-valued and tensor-valued probability density driven diffusion. See respective section for methods abbreviation. Proposed methods are marked by "o" and methods used for comparison is marked by " $\mathrm{x}$ ".

each RGB colour component. In most cases this approach works reasonably well for noise levels $\sigma<70$ when compared to the true underlying noise distribution, but note that the image itself contains noise, hence the estimate can be biased.

In the subsequent chapters each section presents an application and includes evaluation setup, parameter settings, used colour space and results. Note that we make no claim on superiority over all existing techniques for image denoising. For each application we have limited ourselves to methods that are relevant for the considered case. Table 11.1 is provided as a means of an easy overview of compared algorithms. We hope that this will promote additional evaluation of the studied methods. 


\section{Chapter 12}

\section{Scalar-valued applications}

This chapter presents the evaluation of the scalar-valued diffusion formulations considered in previous chapters. Section 12.1 presents the application of enhancing medical images by utilising the visualization function, which in this case, is set to enhance soft tissue. In section 12.2 the mapping function is defined using the channel representation, which yields a diffusion scheme that oversmooths outliers in the image data. The final section, section 12.3 . shows the evaluation of exploiting probability density functions estimated from oversegmentationmaps.

The previous chapter, chapter 11. gives an overview of the evaluation framework. The overview includes used colour representation, image quality metrics and table 11.1 that summarise all evaluated methods for respective application.

\subsection{Non-linear isotropic enhancement of medical images}

This section considers the application of medical image visualization. The evaluation focuses on isotropic diffusion with a mapping function presented in section 5.2 and is denoted as targeted iterative filtering (TIF) [7].

Visualizations in medical imaging are computed by transfer functions, which usually are piecewise linear [75. However, sufficiently similar functions produce visualizations that are visually indistinguishable. We use combinations of sigmoid functions 5.28), see figure 12.1, since they are $\mathcal{C}^{\infty}$ differentiable.

The derivatives of the mapping function, $m$, are computed analytically. However, before evaluating the derivatives of $m(u)$, the signal $u$ is regularized with a small Gaussian filter. To remedy the fact that different propagation speeds are obtained for different slopes of the mapping function, derivatives are normalized to attain a maximum of 1 . 
SSIM

\begin{tabular}{cccccc}
\hline$\sigma$ & $\hat{\sigma}_{m}$ & LD & PM & TIF & AD \\
\hline 5 & 51.19 & $0.89 \pm 0.005$ & $0.92 \pm 0.004$ & $0.93 \pm 0.003$ & $0.94 \pm 0.003$ \\
10 & 102.38 & $0.84 \pm 0.004$ & $0.87 \pm 0.005$ & $0.89 \pm 0.005$ & $0.87 \pm 0.006$ \\
15 & 153.56 & $0.82 \pm 0.006$ & $0.83 \pm 0.005$ & $0.86 \pm 0.006$ & $0.82 \pm 0.005$ \\
PSNR & & & & & \\
\hline$\sigma$ & $\hat{\sigma}_{m}$ & LD & PM & TIF & AD \\
\hline 5 & 51.19 & $28.44 \pm 0.37$ & $30.82 \pm 0.56$ & $30.76 \pm 0.51$ & $32.18 \pm 0.72$ \\
10 & 102.38 & $25.92 \pm 0.45$ & $27.68 \pm 0.49$ & $28.13 \pm 0.53$ & $27.88 \pm 0.49$ \\
15 & 153.56 & $24.81 \pm 0.54$ & $25.74 \pm 0.53$ & $26.82 \pm 0.59$ & $25.38 \pm 0.51$ \\
\hline
\end{tabular}

Table 12.1: Error measures SSIM and PSNR computed from $400 \mathrm{CT}$ images to evaluate targeted iterative filtering (TIF). The estimated noise in the transformed domain, $\hat{\sigma}_{m}$, was computed according to 4.11.

\subsubsection{Evaluation setup}

The methods investigated are, the novel targeted filtering scheme (TIF), linear diffusion (LD), non-linear diffusion (PM) and tensor-based image diffusion (AD). In the evaluation, we add zero mean Gaussian noise of standard deviation 5, 10, and 15 in an 8 bit representation to a set of computed tomography (CT) images (see section 11.3. In the CT reconstruction the logarithm of the data is taken, thus multiplicative noise can be modelled as additive noise.

In the experiments the mapping function is set to visualize soft-tissue by defining the endpoints, in Hounsfield units, as $u_{1}=864$ and $u_{2}=1264$ in 5.28). Figure 12.1 shows the corresponding mapping function. Using the result (4.11, and with the selected endpoints, the noise levels in the visualization domain is $\hat{\sigma}_{m}=\{51.19,102.38,153.36\}$.

\subsubsection{Parameter settings}

All diffusion methods were set to iterate the solution until the peak signal-to-noise ratio (PSNR) value no longer increases. The step length was set to 0.05 for all methods except for the proposed method which utilises the slope of the mapping function, i.e., $\lambda=\min \left(1 /\left(u_{2}-u_{1}\right), 0.25\right)$. The maximum step length is set to 0.25 , this ensures stability in the case of linear diffusion [95].

\subsubsection{Results}

Table 12.1 shows the SSIM and PSNR values obtained in the visualization domain for a dataset of $400 \mathrm{CT}$ images. Comparing the filtering methods with respect to the error measures, the error values are in favour of the proposed targeted filtering method, TIF, for higher noise levels. 


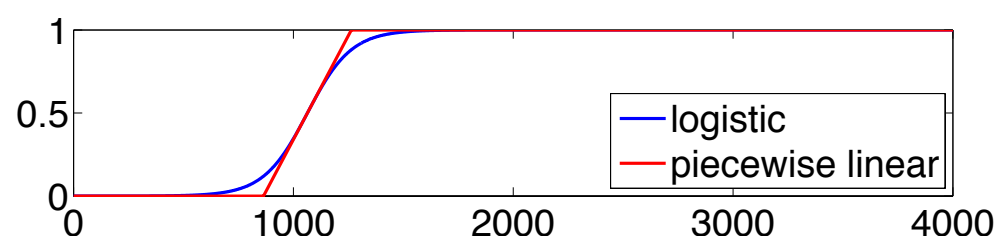

Figure 12.1: Example of mapping function for soft tissue with endpoints $u_{1}=864$ and $u_{2}=1264$ in Hounsfield units.

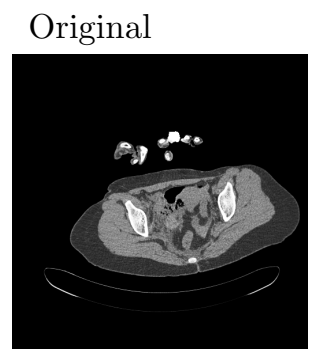

LD
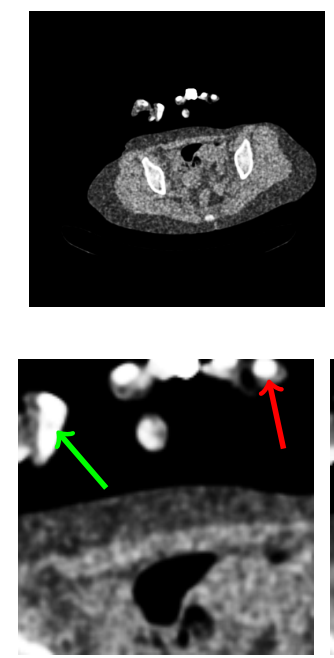

Noisy

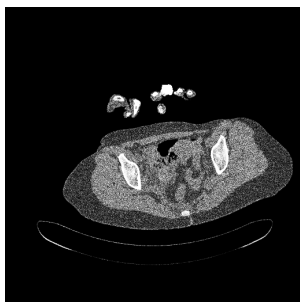

$\mathrm{PM}$
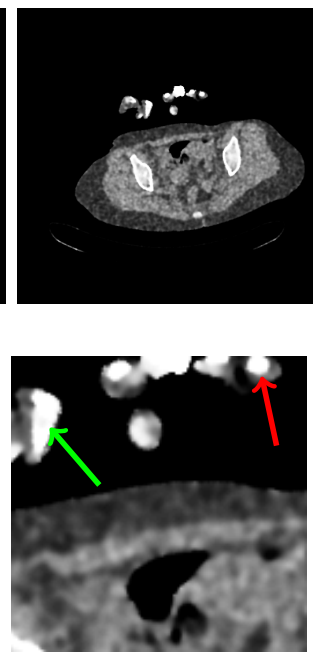

Vis. Original

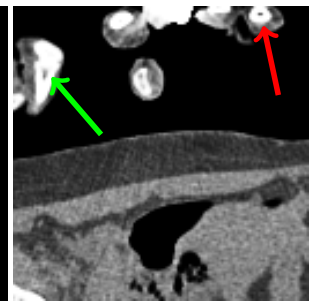

TIF
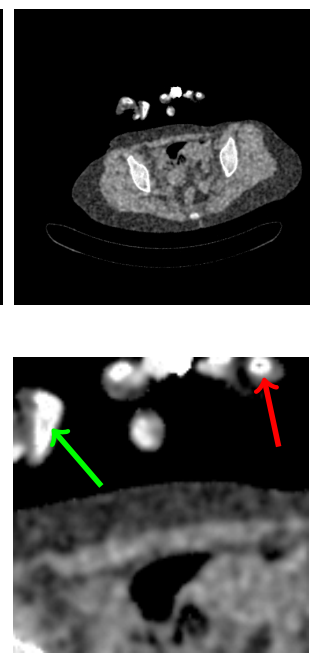

Vis. Noisy

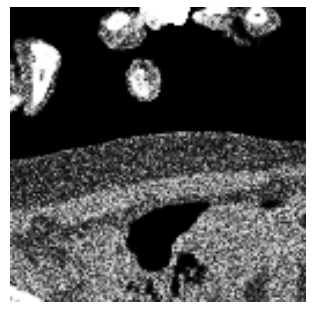

$\mathrm{AD}$
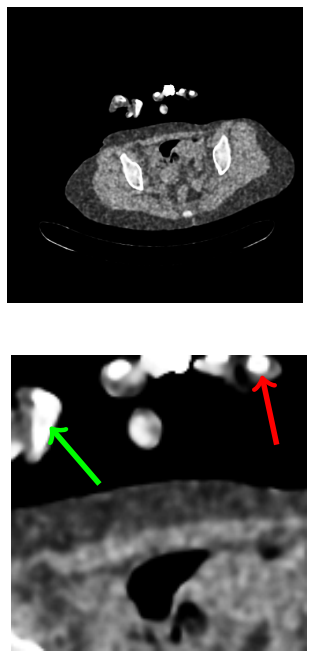

Figure 12.2: Result of processing slice 350 with noise level $\sigma=10$ in the datadomain for a corresponding 8 bit quantization level. Note that the areas, indicated by the arrows, show better preservation of the regions of interest in TIF compared to $\mathrm{LD}, \mathrm{PM}$ and $\mathrm{AD}$. 
Here it is important to note the fundamental difference between TIF and PM. The performance of PM is determined based on the estimation of a contrast parameter for the non-linear mapping function, whereas TIF is not. The only parameter required to be determined in TIF (as with all iterative methods) is the stopping time to avoid trivial solutions. Thus, disregarding the stopping time, TIF is a non-parametric non-linear diffusion scheme which behaves similarly to PM diffusion.

Figure 12.2 visualize the corresponding images of slice 350 with noise level $\sigma=10$. In addition to the visualizations, respective details are depicted. Visually, the proposed diffusion scheme produces superior results close to edges compared to LD and PM diffusion indicated by the arrows in both figures. LD oversmooths the image and PM simply retains noise close to edges. AD preserves edges well and produces high PSNR and SSIM values but approximately homogeneous regions appear oversmoothed. In figure 12.2 it is clear that regions indicated by the arrows have been retained in TIF whereas the other diffusion techniques have removed the structure.

The next section presents the evaluation of the channel-based regularization.

\subsection{Channel-based regularization}

This section evaluates the proposed linear channel diffusion (LCD) and the nonlinear channel diffusion (NLCD) from chapter 7 on a set of greyscale images.

\subsubsection{Evaluation setup}

The aim of the evaluation is to compare the proposed LCD and NLCD schemes especially to diffusion schemes and channel smoothing as we introduced an extension of these methods. Current state-of-the-art denoising methods are included as well. We compare to linear diffusion (LD) and non-linear diffusion (NLD) as introduced by Perona and Malik [72. Furthermore, we consider the tensor-driven anisotropic diffusion scheme (AD) 95]. Besides channel smoothing (CS) 34, median filtering $(\mathrm{MF})$ is included as a method well suited for impulse noise. BM3D [27, currently one of the best methods for filtering Gaussian noise is also included. Finally, two implementations of non-local means (NLM) are considered. We used the original, pixel based implementation [22] as well as the patch based variant [21].

Standard images such as "Cameraman" are used as well as images from the Berkeley image database [65] commonly used for segmentation purposes. Since we are interested in investigating the case of a mixture of noise models we corrupt the images with Gaussian noise as well as impulse noise. Here we consider the presence of $5 \%$ impulse noise and vary the standard deviation of Gaussian noise $\sigma \in\{5,10,15,20,30,40,50\}$.

\subsubsection{Parameter settings}

All methods have been optimised with respect to their parameters. For example, LCD and NLCD were optimised with respect to the number of channels and the 

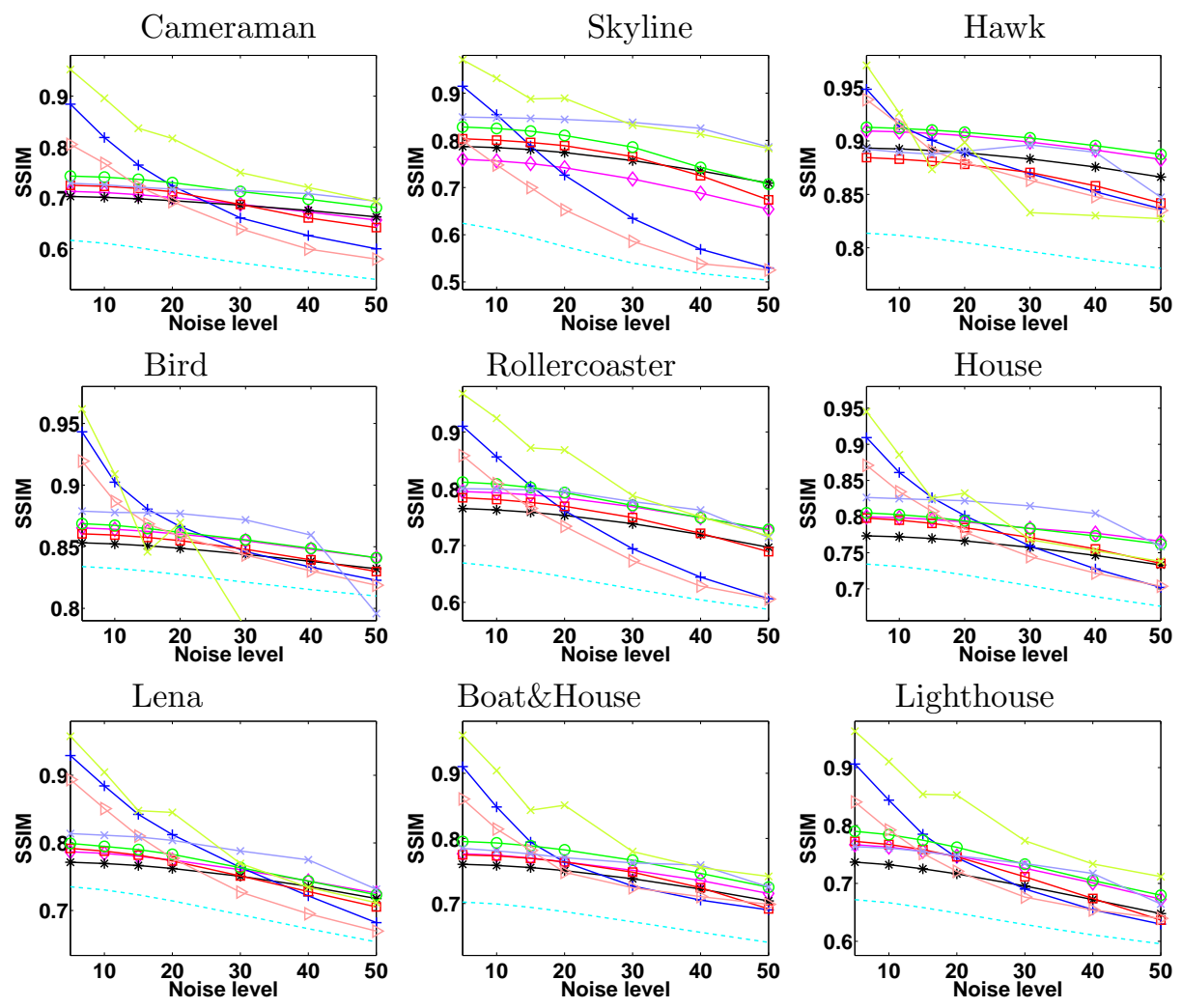

Boat\&House
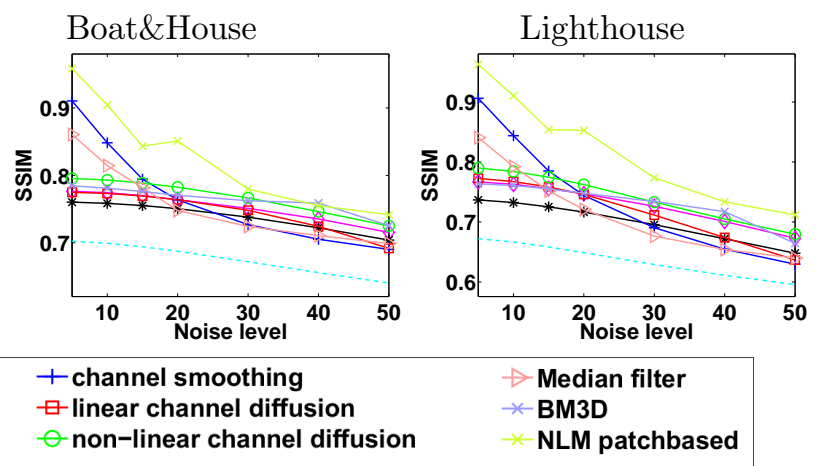

Figure 12.3: Quantitative denoising results of the images Skyline (69007), Hawk (42040), Bird (197027), Rollercoaster (235098), Boat \&House (140088) and Lighthouse (228076) from the Berkeley image database [65] and standard test images (Cameraman, House, Lena). Used methods are linear diffusion, NLD [72, AD [95], CS [34], the novel LCD, the novel NLCD, MF [41], BM3D [27] and NLM [21]. SSIM versus different Gaussian noise levels is plotted. 
diffusivity parameter $k$. The edge stopping function $\Psi$, is set as

$$
\Psi(|\nabla u|)=\left(1+\frac{|\nabla u|^{2}}{k^{2}}\right)^{-1} .
$$

Furthermore, the size of the median filter was optimised. For the channel smoothing (CS) we use a box decoding scheme, optimise the number of channels and the variance of the Gaussian filter used for smoothing in each channel. In AD, the mapping of the structure tensor eigenvalues to diffusivities is done using the same edge stopping function as for NLD.

For each method the filtering is continued until the maximum structure similarity index (SSIM) 94 has been reached.

\subsubsection{Results}

Figure 12.3 shows the best obtained SSIM value for each image and considered noise level. The pixel based NLM variant [22] has SSIM values between 0.3 and 0.5 for all images as it poorly reduces impulse noise. To focus on higher SSIM values we do not show values for pixel NLM.

Generally, best denoising results are obtained by the patch version of the nonlocal means, especially for low-level Gaussian noise. BM3D also produces high SSIM. For low Gaussian noise CS shows good results as well. This was expected as channel smoothing, as well as median filtering, are well suited for removing clear outliers. The NLCD gives quite high SSIM for higher Gaussian noise levels.

For few special cases we observe that NLCD performs best, even better than the state-of-the-art denoising methods BM3D and NLM, which is unexpected, see, e.g., result for the hawk image. In all cases NLCD is comparable and most times better than the other diffusion-based methods, e.g., in the Cameraman. As soon as Gaussian noise of $\sigma=15$ or $\sigma=20$ has been added to the image the NLCD also outperforms CS.

In figure 12.4 visualization of certain image close-ups can be seen. The CS and MF show a "comic book"-like behaviour, maintaining the main edges well but details are lost as can be seen in the face or the camera. The pixel based NLM cannot handle the impulse noise at all. Better preservation of details is achieved using AD or NLD. Good results show the patch based NLM and BM3D. However, for patch NLM the image still shows some Gaussian noise and for BM3D still some details are lost. The proposed LCD shows an improvement compared to $\mathrm{CS}$ and MF, but due to its linear form the edges are smeared. Visually, better results compared to CS and diffusion methods are obtained with NLCD. It keeps the details and it is able to handle impulse noise as well as Gaussian noise. 
Cameraman 0.2692

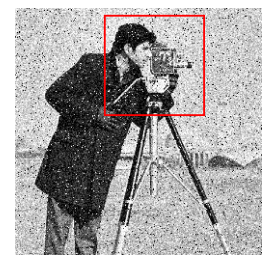

Hawk 0.1460

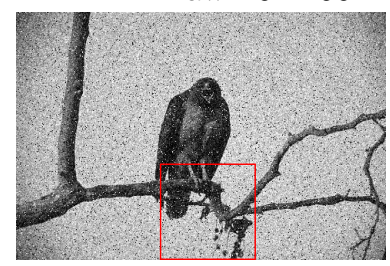

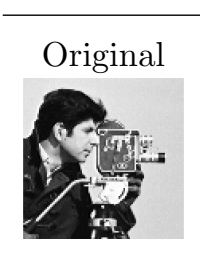

CS

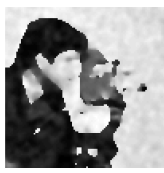

0.6606

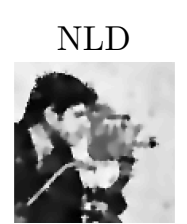

0.6874

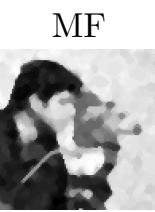

0.6393

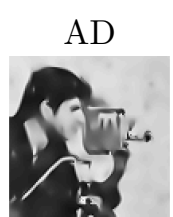

0.6858

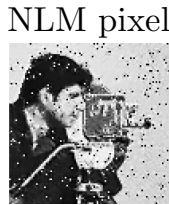

0.3753

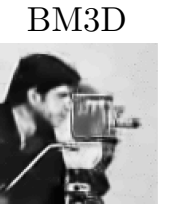

0.7145

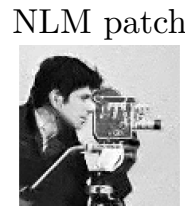

0.7408

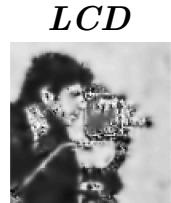

0.6871

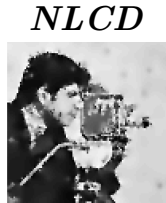

0.7126
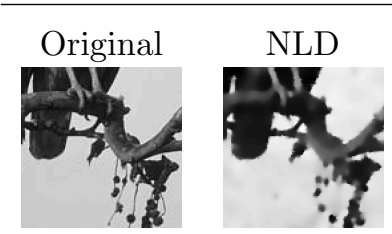

0.8989
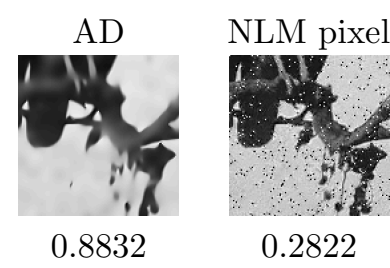

0.2822
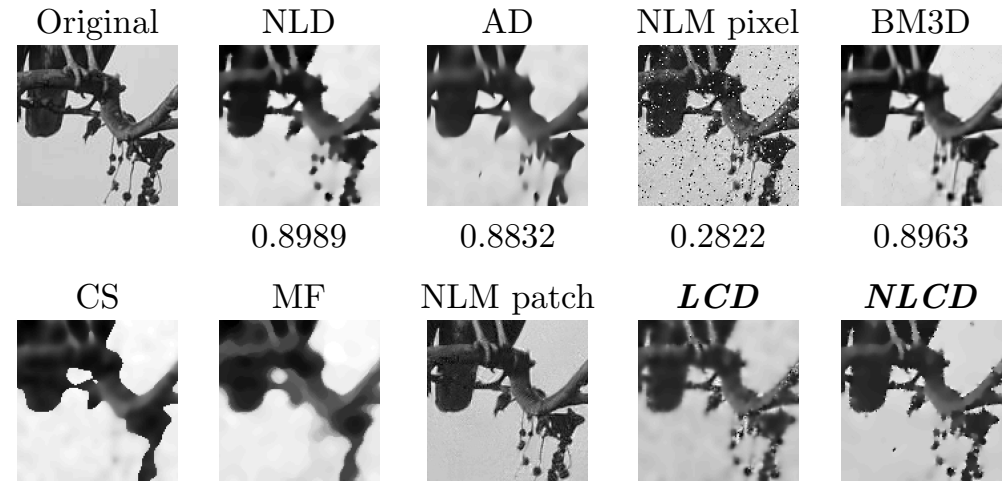

0.8694

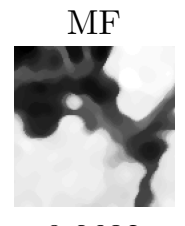

0.8632

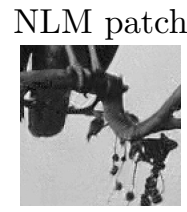

0.8329

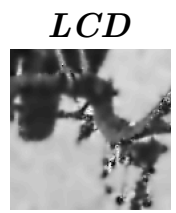

0.8705

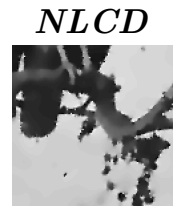

0.9028

Figure 12.4: Final denoising results and corresponding SSIM values obtained when the images were corrupted by 30 standard deviations of noise. See figure 12.3 for details. 


\subsection{Enhancement via density estimates}

This section evaluates the method density driven diffusion, D3 presented in chapter 6, on a set of colour images and standard greyscale test images.

\subsubsection{Evaluation setup}

The compared methods are CBM3D [28, 27] and NLM [22]. Also the extended anisotropic diffusion, EAD [2], presented in section 4.3 is included. The implementation of each method was obtained from their respective authors.

Colour images indexed 8143 (Owl), 87065 (Lizard), 175043 (Snake) and 208001 (Mushroom) available through the Berkeley segmentation dataset [65] were used in section 13.1 thus we evaluate D3 also on these images. It has been shown in previous works that colour image denoising in the RGB colour space is suboptimal due to high correlation of the colour components [8, 2]. The approach for applying D3 on colour images is to use the segmentation method of [66] (described in section 12.3.3. The produced segmentation map conforms to homogeneous colour regions. Then we use the segmentation map to estimate density functions in the colour opponent space $O_{1}, O_{2}$ and $O_{3}$ defined in $11.1-11.3$, since the spatial position of the pixels has not changed due to the colour transformation.

\subsubsection{Parameter settings}

With regards to the parameter selection of EAD, it has been shown that, modifying the estimate of the diffusion parameter to scale with the colour transform $k_{O_{1}}=10^{-1} k$ and $k_{O_{2} O_{3}}=10 k$, yields an improved result compared to using $k$ without any scaling (see [2] and section 13.1). The diffusivity parameter $k$ was estimated using 11.7). The motivation for using different diffusion parameters in the different channels is that filtering in the average grey-value channel primarily affects the image noise and hence it is preferable to reduce the $k$-parameter in this channel. However, one must be careful since noise may be considered as structure and may be preserved throughout the filtering process. Filtering in the colour opponent components will provide intra-region smoothing in the colour space and hence colour artefacts will be reduced with a less structural preserving diffusion parameter.

Since all methods, except D3, rely on global noise standard deviation the noise level is estimated according to the method presented in 33 .

\subsubsection{Sensitivity Analysis}

Since the filtering performance will depend on how accurately the estimated density functions represent the local image regions, it is of interest to understand how sensitive the parameter selection of the used segmentation method is. The oversegmentation is controlled by two parameters: the "clique size" that determines the initial size of the segments and a the "clique cost" which is a sensitivity parameter. Depending on the image content different values of these parameters will be required. Typically for images with large homogeneous regions, larger segments 

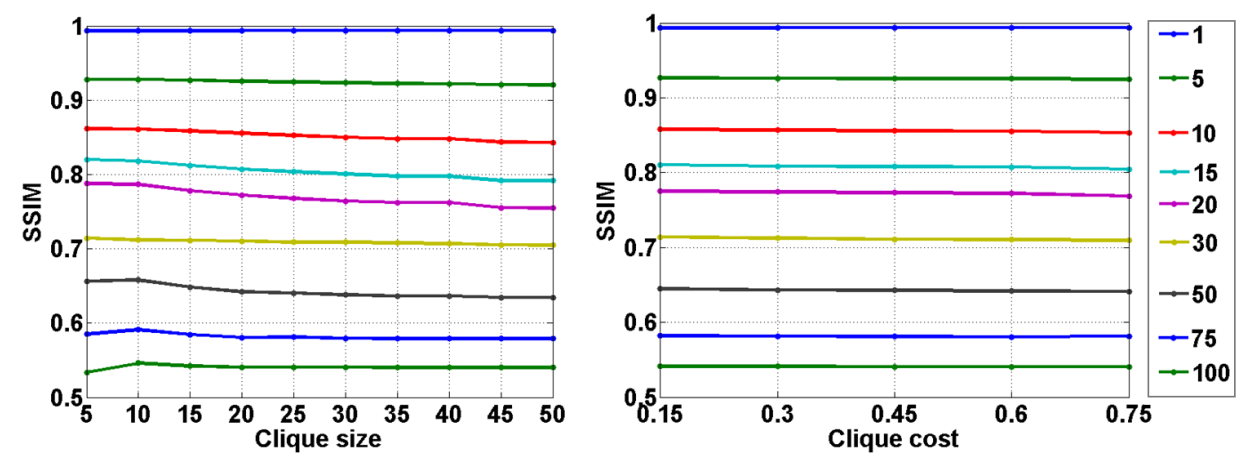

Figure 12.5: Analysis of SSIM values for varying segmentation sizes for a fixed sensitivity value (left) and evaluation of the sensitivity parameter for a fixed segment size (right) as a function of different noise levels.

and a more rigid sensitivity parameter suffice. If the scene is highly textured then smaller segments are preferable. If noise is present then the sensitivity parameter needs to be set not to model the noise as structure.

In figure 12.5 (left), by setting the sensitivity parameter to 0.3 the segment size parameter is plotted against the obtained error value for 9 different noise levels in the range 1 to 100. The noise is expressed in an 8 bit quantization. In figure 12.5 (right) the segment size is set to a fixed value $(10 \times 10$ pixels $)$ and the sensitivity parameter is plotted against the corresponding SSIM value. In both cases, the SSIM values are averaged over all the greyscale images seen in figure 11.2 with the corresponding segmentation sizes and sensitivity coefficients. Figure 12.5 shows that the selection of parameters for a large number of noise levels is not critical for the performance of the density driven diffusion. This illustrates that the filtering method is robust in terms of parameter selection.

\subsubsection{Results}

The obtained SSIM values for the greyscale images in figure 11.2 can be seen in figure 12.7. The differences in SSIM value are on average not significant and the performance of respective method is correlated with the image content. Observe that BM3D 27] performs well in images with large homogeneous regions such as the "House" image.

The result of the colour image evaluation is seen in figure 12.8 . The poor error value of NLM and CBM3D 28] particularly visible in figure 12.6 (a) is due to inaccurate estimation of the noise, thus illustrating that they depend heavily on the accuracy of any noise estimation technique to define their filtering parameters. The proposed method D3, compares well with the tensor-based EAD for the investigated colour images. Here we point out that the EAD is a local tensorbased method estimating the local orientation, thus achieving adaptive filtering. In contrast, D3 is a semi-local method considering the density functions estimated from each segment of the segmentation map independently of the estimated noise. Example images of the denoising result can be seen in figure 12.6 , indicated for 


Noisy $\quad$ CBM3D [28] NLM [22] $\quad$ EAD [2] $\quad$ D3

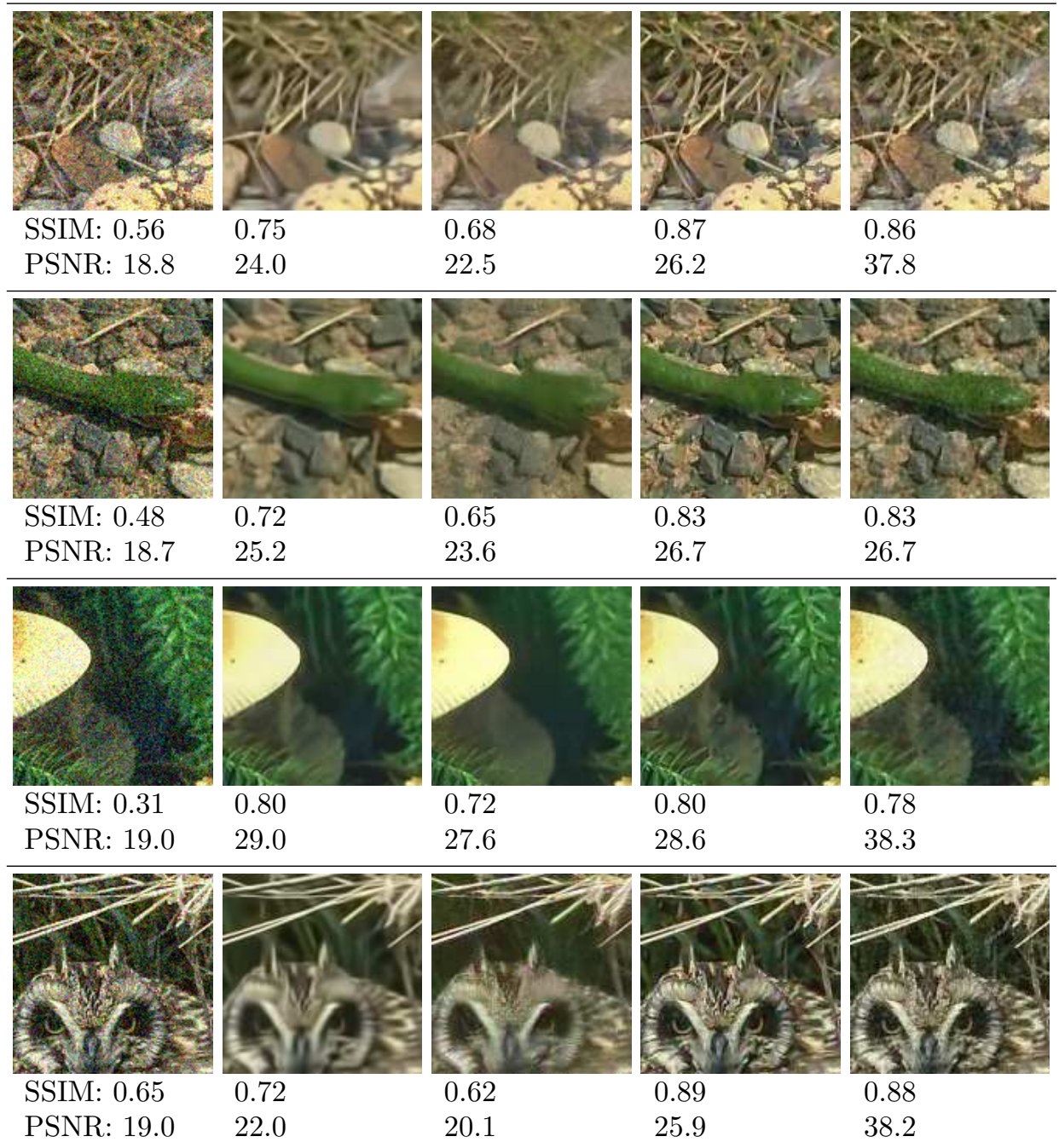

Figure 12.6: Colour image denoising where the images were corrupted by 30 standard deviation noise. From top to bottom: Lizard, Snake, Mushroom and Owl.

each image is the corresponding SSIM and PSNR values at noise level 30.

For images with many high-frequency components such as branches, leaves and feathers it is clear that the local method EAD performs well preserving these structures, particularly visible in the Owl image. CBM3D and NLM have an advantage in images which contain many similar patches, e.g., the Mushroom image. The filtering result obtained by D3 appears crisp and no apparent oversmoothing is visible in the Owl and the Snake as is for the CBM3D and NLM. Also the mean-shift (MS) method [26] was tested but produced very poor results and for that reason it is not illustrated here. 


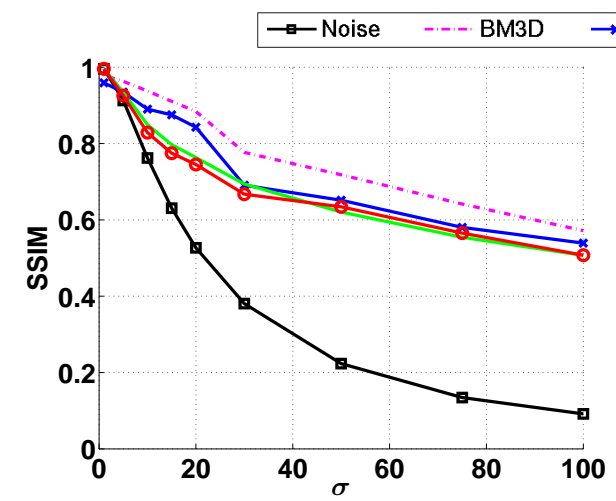

(a) Barbara

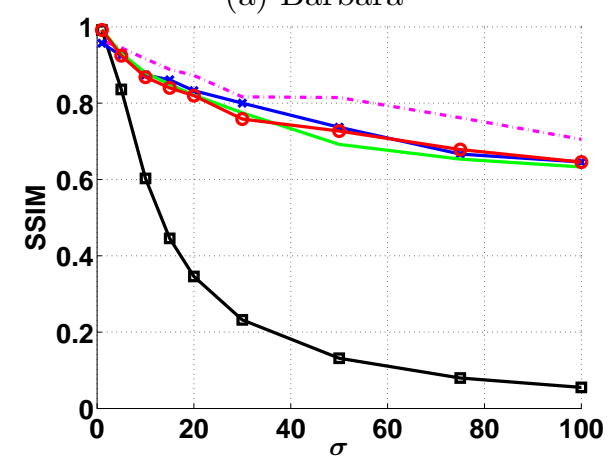

(c) House

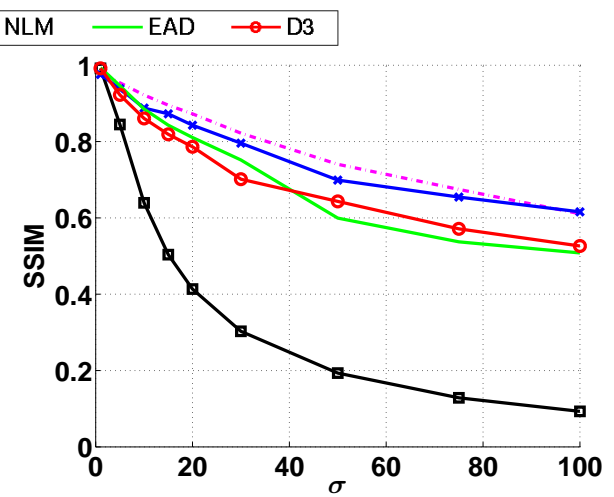

(b) Cameraman

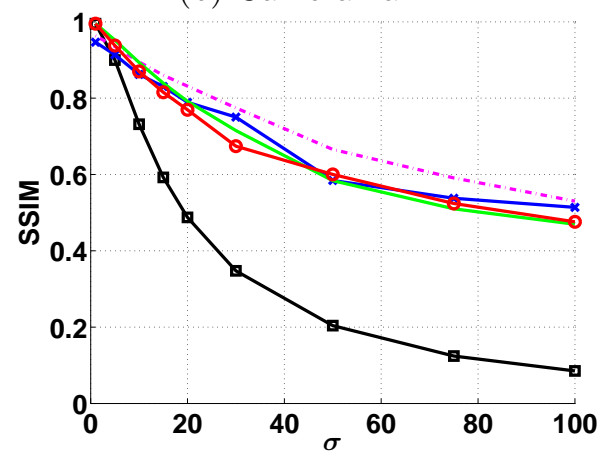

(d) Boat

Figure 12.7: Evaluation of density driven diffusion, D3, on a set of grayscale images. In this test case BM3D performs well for each considered noise level. The label "Noise" corresponds to the image baseline noise. NLM, D3 and EAD has, in the case of grayscale images, very similar performance. 


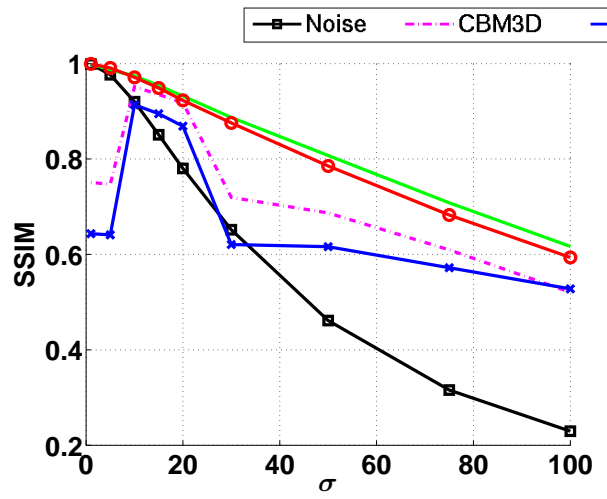

(a) Owl

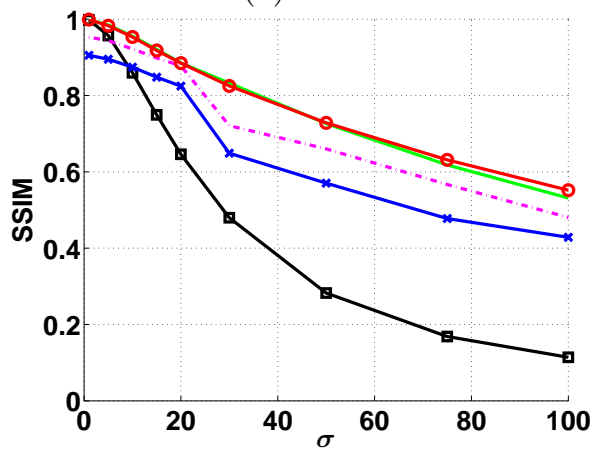

(c) Snake

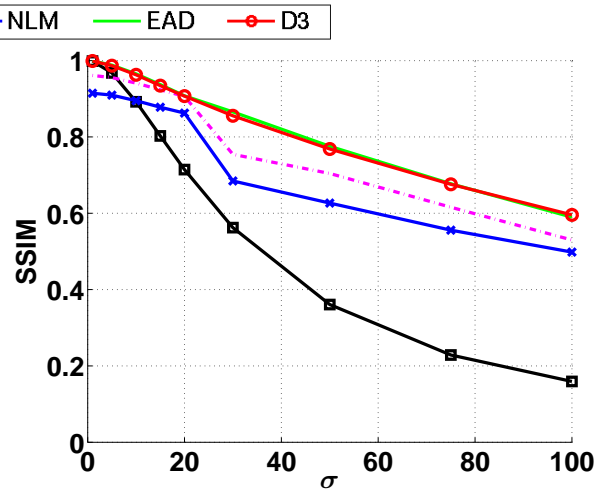

(b) Lizard

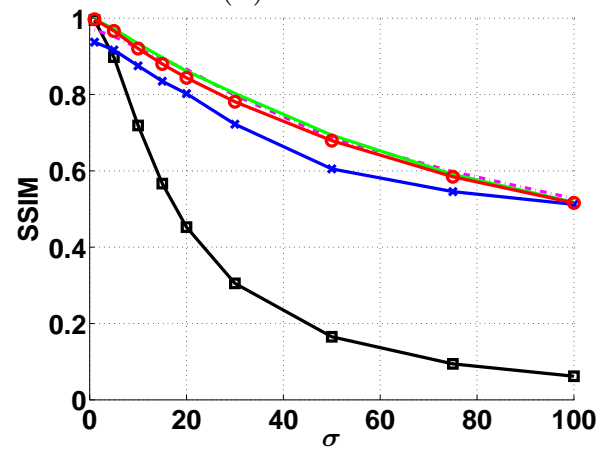

(d) Mushroom

Figure 12.8: Evaluation of density driven diffusion, D3, on a set of colour images. In this case, BM3D does not perform well and D3 shows a comparable result to EAD. "Noise" corresponds to the image baseline noise. 


\section{Chapter 13}

\section{Tensor-valued applications}

This chapter presents the evaluation of the tensor-valued diffusion methods introduced in previous chapters. Section 13.1 presents the evaluation of the extended anisotropic diffusion framework. In section 13.2 the gradient energy total variation framework is evaluated for both greyscale and colour images and section 13.3 presents a significance analysis. The aim is to analyse if the mapping function, defined from an oversegmentation map, does give an improved performance with respect to the error measures.

Chapter 11 gives an overview of the evaluation framework. The overview includes used colour representation and image quality metrics. Table 11.1 summarises all evaluated methods for respective application.

\subsection{Extended anisotropic diffusion}

This section evaluates extended anisotropic diffusion (EAD) introduced in chapter 4. Definition 1 .

\subsubsection{Evaluation setup}

The EAD scheme in Definition 1 consists of two divergence terms with tensors $D_{1}$ and $D_{2}$. The divergence term containing $D_{1}$ is implemented by using the nonnegativity scheme described in 95 ] resulting in a term $A(u)$. The other divergence term is implemented using the approach in 98 described by $B(u)$. In an explicit discrete setting the iterative update equation become

$$
u^{i+1}=u^{i}+\lambda\left(A\left(u^{i}\right)+B\left(u^{i}\right)\right),
$$

where $\lambda$ is the step length for each iteration $i$.

We use colour images from the Berkeley segmentation dataset [65], more specifically images 87065, 175043, 208001, 8143, being denoted as Lizard, Snake, Mushroom and Owl (see figure 13.1). All images predominately contain scenes from 
nature and hence large number of branches, leafs, grass and other commonly occurring structures. The estimated noise levels for the different images can be seen in table 13.1. Gaussian noise of 5, 10, 20, 50 and 70 standard deviations (std) is added to the images. Before denoising, the noisy image is clipped to fit the 8 bit image value range.

To illustrate the capabilities of the proposed technique it is compared to three state-of-the-art denoising techniques, namely trace-based diffusion (TR) [33] (see (3.19), diffusion in the RGB colour space [96] and the colour version of BM3D 28. Code was obtained from the authors of TR-diffusion and BM3D, whereas the RGB-colour space diffusion was reimplemented based on [96] and [95] (cf. p. $95)$, the $k$-parameter in this case is set to the value obtained from 11.7 . We performed the EAD filtering in the colour opponent space 11.1)-(11.3).

\subsubsection{Parameter settings}

The EAD diffusivity parameter, $k$, is set as described in section 12.3 .2 and the diffusivity function is set as the negative exponential function (3.17). The step length for EAD and TR was manually scaled so that the images in figure 13.1 are obtained after approximately $1 / 2$ of the maximum number iterations (100).

\subsubsection{Results}

For a quantitative evaluation we use the SSIM [94] and the peak-signal-to-noise (PSNR) error measurements shown in table 13.1. To the best of our knowledge there is no widely accepted method to evaluate colour image perception, hence the error measurements are computed for each component of the RGB colour space and averaged. The purpose here is not to claim the superiority of any technique, rather it is to illustrate the advantages and disadvantages of the different filter methodologies in various situations. The error measurements SSIM and PSNR values are given to illustrate the strengths of the compared denoising techniques, results are shown in table 13.1

It is clear from table 13.1 that EAD performs well in terms of SSIM and PSNR values compared to the other state-of-the-art denoising techniques. However, for images (such as the Mushroom image) with large approximately homogeneous regions, $\mathrm{BM} 3 \mathrm{D}$ is the favoured denoising technique.

This is due to many similar patches which the algorithm averages over to reduce the noise variance. On the other hand, diffusion techniques perform better in high-frequency regions due to the local description of the tensor. This is also depicted in figure 13.1 where the diffusion techniques preserve the image structure in the Lizard, Snake and the Owl image, whereas BM3D achieves perceptually good results for the Mushroom image. 


\begin{tabular}{|c|c|c|c|c|c|c|c|c|c|}
\hline \multirow{2}{*}{ Noise } & \multicolumn{5}{|c|}{ SSIM } & \multicolumn{4}{|c|}{ PSNR } \\
\hline & Est & TR & EAD & RGB & BM3D & $\mathrm{TR}$ & EAD & RGB & BM3D \\
\hline \multicolumn{10}{|c|}{ Lizard } \\
\hline 5 & 17.0 & 0.99 & 0.99 & 0.98 & 0.97 & 38.0 & 38.0 & 35.2 & 34.1 \\
\hline 10 & 19.2 & 0.96 & 0.96 & 0.94 & 0.95 & 32.7 & 32.8 & 30.6 & 32.3 \\
\hline 20 & 24.2 & 0.90 & 0.91 & 0.87 & 0.92 & 27.3 & 27.8 & 26.7 & 29.3 \\
\hline 50 & 57.1 & 0.75 & 0.77 & 0.68 & 0.73 & 22.6 & 23.5 & 22.0 & 23.2 \\
\hline 70 & 67.1 & 0.65 & 0.70 & 0.59 & 0.66 & 20.8 & 21.8 & 20.5 & 21.7 \\
\hline \multicolumn{10}{|c|}{ Snake } \\
\hline 5 & 15.5 & 0.98 & 0.98 & 0.97 & 0.96 & 38.3 & 38.3 & 35.3 & 34.6 \\
\hline 10 & 18.1 & 0.95 & 0.95 & 0.92 & 0.94 & 33.0 & 33.1 & 30.9 & 32.6 \\
\hline 20 & 23.5 & 0.87 & 0.88 & 0.83 & 0.90 & 27.7 & 28.4 & 27.3 & 29.7 \\
\hline 50 & 53.9 & 0.69 & 0.73 & 0.63 & 0.69 & 23.2 & 24.3 & 23.0 & 24.4 \\
\hline 70 & 65.4 & 0.59 & 0.64 & 0.54 & 0.61 & 21.5 & 22.7 & 21.6 & 22.9 \\
\hline \multicolumn{10}{|c|}{ Mushroom } \\
\hline 5 & 9.9 & 0.97 & 0.97 & 0.95 & 0.96 & 38.7 & 38.9 & 36.9 & 38.2 \\
\hline 10 & 13.9 & 0.92 & 0.93 & 0.89 & 0.93 & 33.8 & 34.3 & 32.8 & 35.1 \\
\hline 20 & 21.1 & 0.83 & 0.86 & 0.79 & 0.88 & 29.3 & 30.4 & 29.2 & 31.8 \\
\hline 50 & 49.0 & 0.66 & 0.70 & 0.58 & 0.70 & 25.1 & 26.0 & 24.7 & 26.4 \\
\hline 70 & 60.4 & 0.57 & 0.62 & 0.52 & 0.63 & 23.0 & 23.8 & 22.8 & 24.1 \\
\hline \multicolumn{10}{|l|}{ Owl } \\
\hline 5 & 61.4 & 0.99 & 0.99 & 0.98 & 0.78 & 36.9 & 37.0 & 34.6 & 23.2 \\
\hline 10 & 19.8 & 0.97 & 0.97 & 0.95 & 0.96 & 33.0 & 33.0 & 30.1 & 32.0 \\
\hline 20 & 25.7 & 0.93 & 0.93 & 0.88 & 0.93 & 27.8 & 28.0 & 25.8 & 28.7 \\
\hline 50 & 65.1 & 0.80 & 0.81 & 0.68 & 0.72 & 22.6 & 23.0 & 20.5 & 21.8 \\
\hline 70 & 71.2 & 0.71 & 0.73 & 0.59 & 0.66 & 20.4 & 21.0 & 18.8 & 20.5 \\
\hline
\end{tabular}

Table 13.1: Obtained SSIM and PSNR values when evaluating extended anisotropic diffusion (EAD) on a set of colour images. With regards to the SSIM value, EAD is favoured in most cases. However, with respect to the PSNR value, BM3D is the preferred method for the considered test images. 


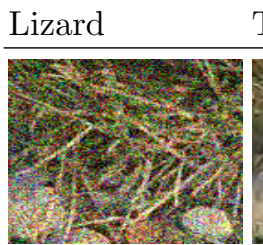

TR 8

$\mathrm{EAD}$

RGB 96

BM3D 28

PSNR:
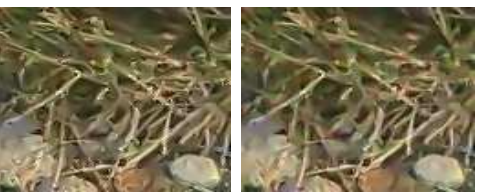

SSIM:

22.6

23.5

22.0

23.2

0.77

0.68

0.73

Snake
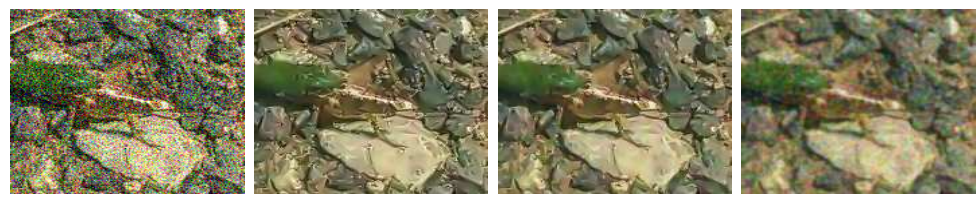

PSNR:

23.2

24.3

23.0

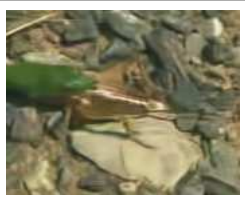

SSIM:

0.69

0.73

0.63

24.4

0.69

Mushroom
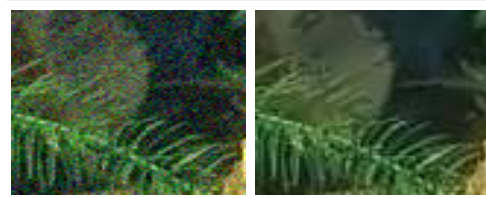

PSNR:

29.3

SSIM:

0.83
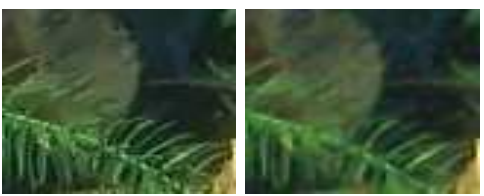

30.4

29.2

0.86

0.79

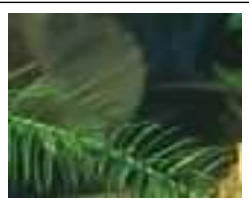

Owl
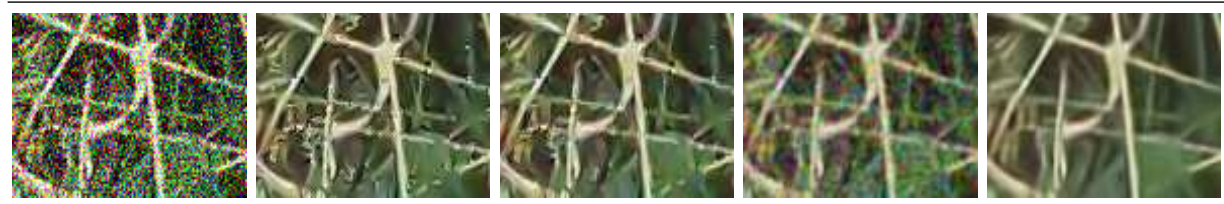

PSNR:

20.4

21.0

18.8

20.5

SSIM:

0.71

0.73

0.59

0.66

Figure 13.1: Zoomed patches from the respective test images. The images Lizard and Snake are results from denoising additive Gaussian noise of 50 standard deviations. The Mushroom image was corrupted by 20 standard deviations of noise, and Owl was corrupted by 70 standard deviations of noise. 


\subsection{Gradient energy total variation}

In this section we evaluate the gradient energy total variation scheme (GETV) introduced in chapter 9 without a mapping function. The evaluation of GETV with a mapping function is done in section 13.3 which also includes a comparison with respect to total variation with a mapping function presented in section 5.3 .

\subsubsection{Evaluation setup}

The GETV is evaluated and compared to extended anisotropic diffusion (EAD) 2 and a state-of-the-art primal-dual implementation of the Rudin-Osher-Fatemi [79] total variation model (TV) $[23]^{1}$. The test data was corrupted with Gaussian additive noise of standard deviations $5,10,15$ and 20 . In this work we use the CIELAB colour space transform, however other choices of colour spaces are possible as investigated in [2].

Test data that we considered for the general evaluation are twofold. First we consider a number of standard greyscale images Barbara, Boat, Cameraman (cman), House and Lena, each image is of size $256 \times 256$. The other dataset is the Berkeley database [65, where we randomly choose 50 colour images each of size $481 \times 321$.

\subsubsection{Parameter settings}

The E-L equation that we solve is 9.15 but with regularized derivatives, i.e., let $\beta$ denote regularization with a small positive constant such that the denominators are expressed as $|\nabla u|_{\beta}=\sqrt{|\nabla u|^{2}+\beta^{2}}$.

To implement GETV we are required to compute third-order derivatives (in the gradient energy tensor) for terms such as $\partial_{x} \Delta u$, and we find that it is appropriate to directly approximate these higher-order derivatives with central differences of the Laplacian. In practice, to avoid numerical instabilities, it is sufficient to regularize the first and second order derivatives with a Gaussian filter of standard deviation $\sigma$ of 8/10. To compute the third-order term a Gaussian filter of standard deviation 3 was suitable for regularization. These filter sizes were kept constant for all images and all noise levels in the experimental evaluation, we fixed $\beta=10^{-4}$.

\subsubsection{Test pattern}

In figure 13.2 we illustrate the behaviour of the three schemes on a radial test pattern of increasing frequency components. The histogram illustrates that the proposed gradient energy total variation (GETV) scheme in essence exhibits fewer large magnitude errors than the other methods, this is marked by the red box. The EAD scheme shows errors in high-frequency areas as illustrated with the magenta colour, whereas standard total variation gives errors for all frequencies due to the tendency to enforce piece-wise constant surfaces.

\footnotetext{
${ }^{1}$ Code (14-02-17) gpu4vision.icg.tugraz . at/index .php?content=downloads .php
} 

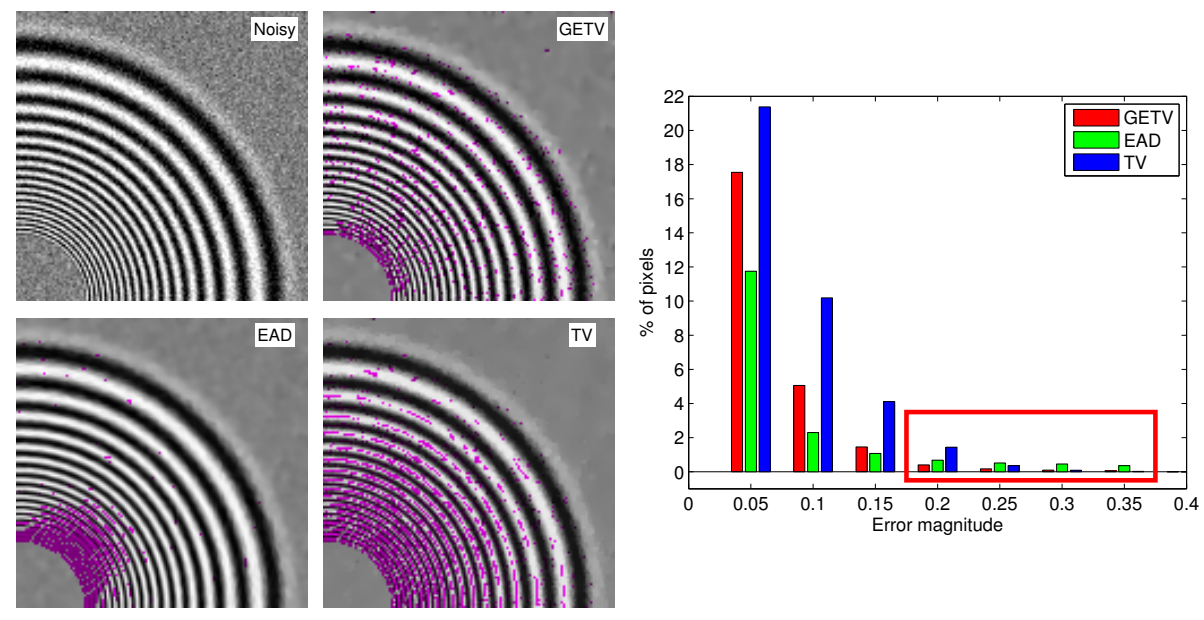

Figure 13.2: A test image corrupted with 20 standard deviation additive Gaussian noise and the corresponding denoised results. Observe that the errors larger than $10 \%$ (magenta) are considerably less concentrated at high frequencies for the GETV than the other methods.

\subsubsection{Evaluation criteria}

Since large homogeneous regions have more impact on the error measures than edges in the image do, we compute a weighted PSNR, W-PSNR, to assess preservation of edges in the images after filtering. The weight is defined to be the trace of the structure tensor and it is applied on the difference between the original and the enhanced image in the computation of the PSNR. Since the trace measures the magnitude of the gradient, the W-PSNR value correlates with a better preservation of edges than the PSNR measure does in relation to the noise-free image.

Image auto-denoising is a method that does not take the noise-free image into account when determining a quality measure, instead it uses the noisy image. To avoid manual parameter tuning the approach was used to optimise the selection of parameters in the different filtering schemes. The exploited metric was proposed in [58, denoted here as A-IQA (auto-image quality assessment). The basic idea of A-IQA is that a high correlation score is obtained if the denoised image has smooth surfaces, but yet preserves boundaries. In the total variation scheme, we select the regularization parameter $\lambda$ from the values $6,8,10,12$ and 14 based on maximum A-IQA.

The diffusivity parameter $k$ for EAD is computed according to (11.7). The same value of $k$ is used in the proposed GETV scheme but scaled with a factor $10^{-1}$. The stopping time for all methods was determined by the maximum A-IQA value. 

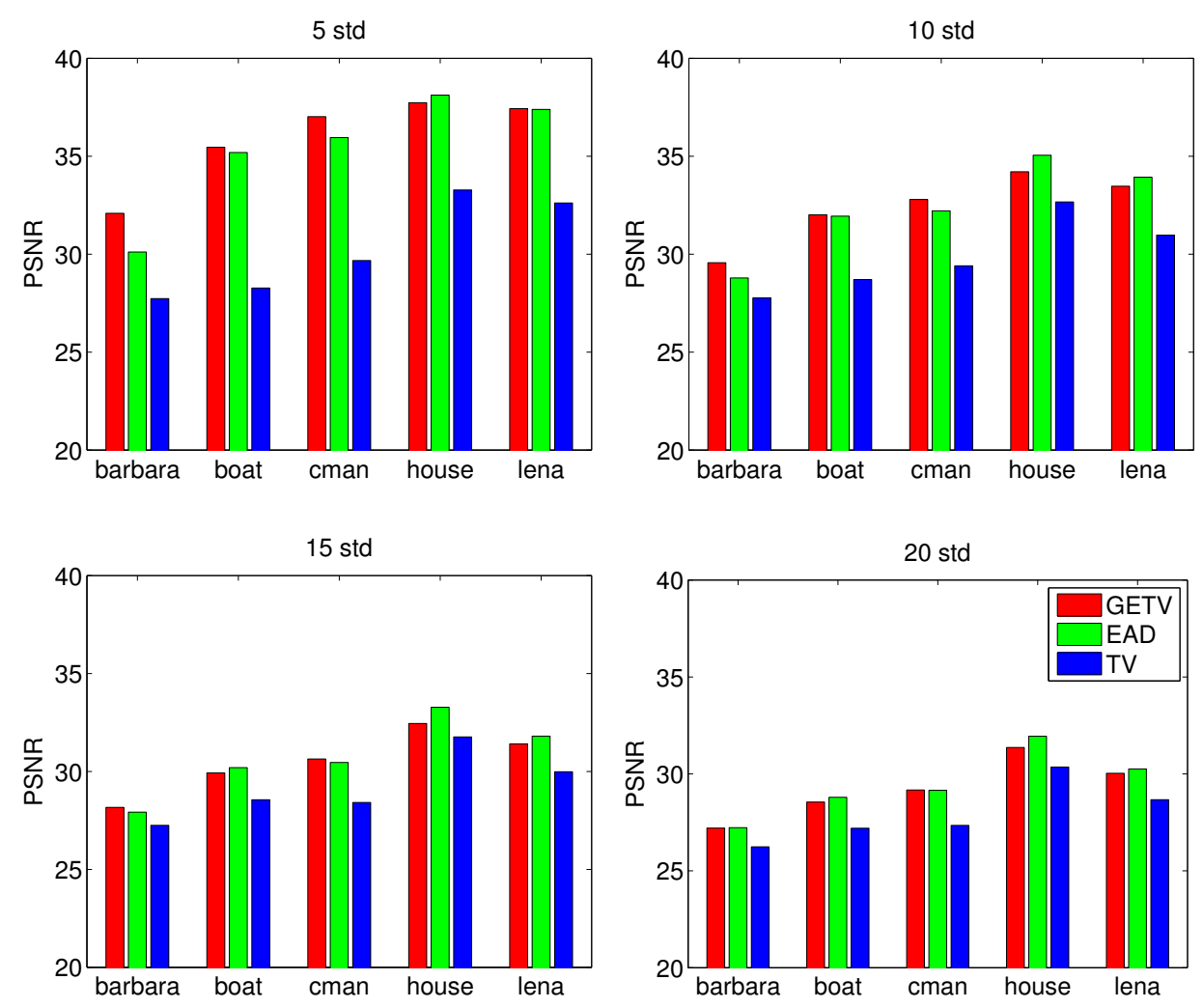

Figure 13.3: The PSNR error measure for when evaluating the gradient energy total variation (GETV) scheme compared to extended anisotropic diffusion (EAD) and total variation (TV) for on grayscale images. Note the poor performance of total variation whereas GETV and EAD shows a similar performance.

\subsubsection{Results}

Figure 13.3 shows the PSNR values obtained for each greyscale image and noise level. Observe that the standard TV formulation does not perform well compared to EAD and GETV in these cases. Figure 13.5 shows close-ups of Cameraman, Boat and Barbara. Note that in all cases the error measures are similar for the AIQA values, however considering the visual quality it is obvious that more details are preserved in GETV, i.e., the presence of sharp edges in the Cameraman image such as the handle of the camera.

With respect to the colour images, figure 13.6 shows examples from the Berkeley dataset and the corresponding error measures are given in figure 13.4. Comparing EAD and GETV for lower noise levels (5-15 standard deviations) the difference in PSNR and SSIM is at best marginal. However, considering the variance (shown by the vertical bar), GETV is more robust than EAD. In figure 13.6 the visual 

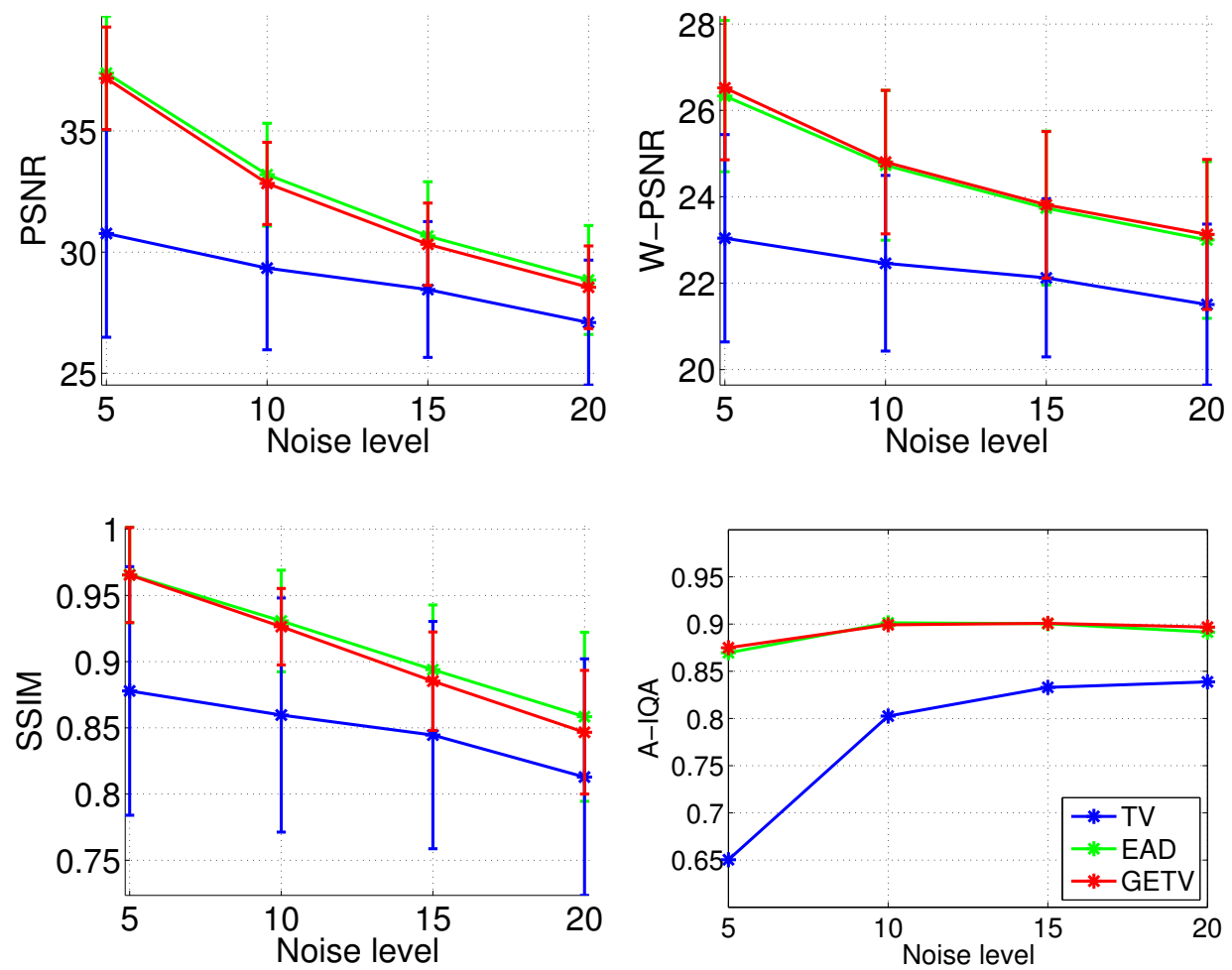

Figure 13.4: Mean and standard deviation of error measures for the Berkeley dataset. Error measures, a higher W-PSNR indicates better recovery of highfrequency regions. The visual appearance for selected images is shown in figure 13.6

differences can be seen for some selected images. Note that it is primarily in the high-frequency regions that GETV excels, consider, e.g., the clarity of the document and the visibility of waves. For both greyscale and colour images, EAD tends to oversmooth the images. Furthermore, it is obvious that the TV-method fails to handle these images when auto-tuning is used. By manually tweaking the regularization parameter of the methods we can improve the error measures for some images, however this approach is infeasible for a large number of images. 


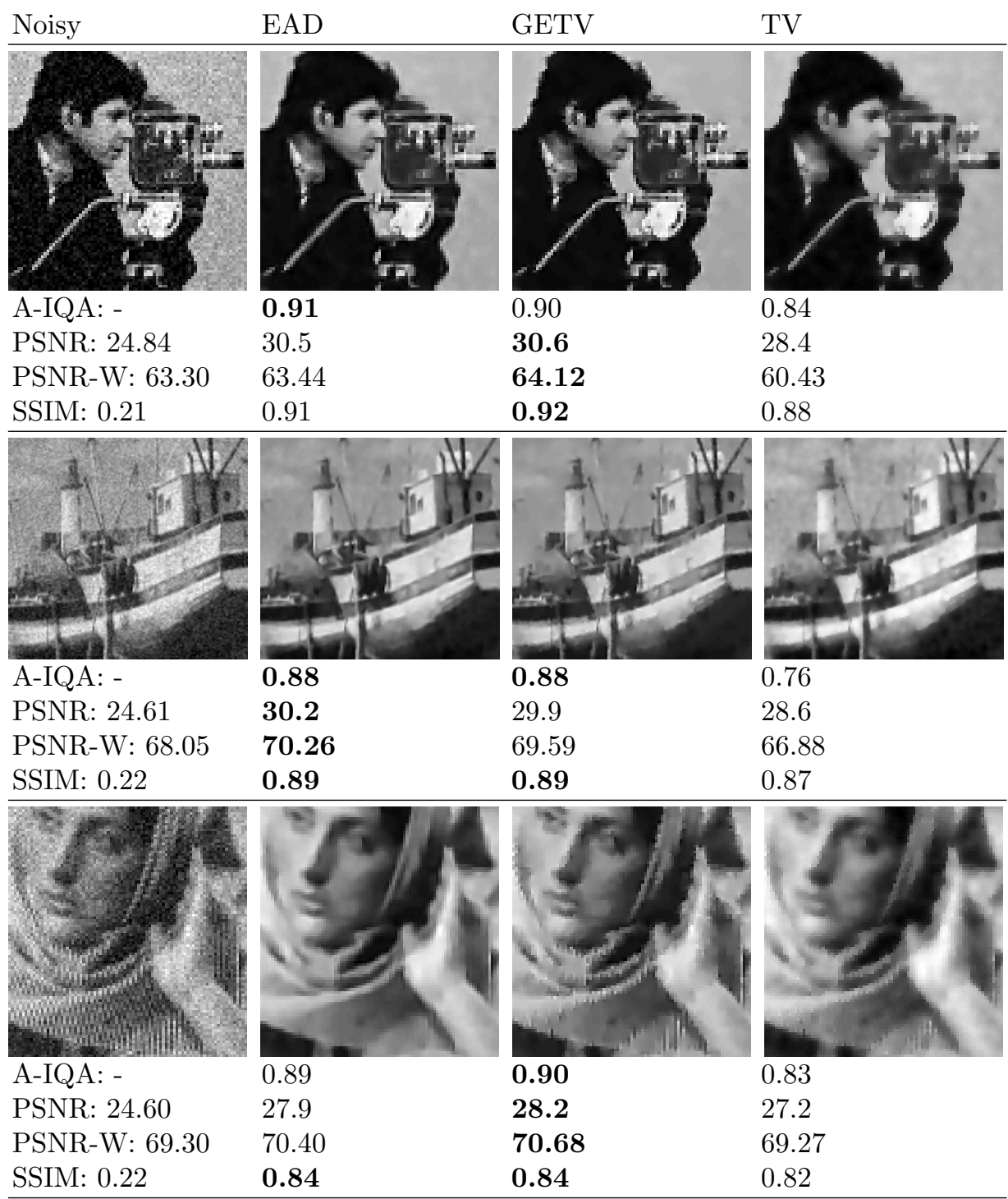

Figure 13.5: Example from the greyscale dataset for 15 standard deviation of noise. GETV shows an improvement over EAD and TV, both in PSNR and preservation of fine image structures such as the camera handle. Also, note that the images obtained with GETV appear less blurry than EAD and TV. 


\begin{tabular}{lllll} 
Noisy & EAD & GETV & \\
\hline
\end{tabular}

Figure 13.6: Results from the Berkeley colour image dataset with 15 standard deviation of noise where GETV excels. Consider particularly the text on the document and the grass behind the horse on the last row. Note that GETV in general preserves more fine details than EAD and TV, which both tends to oversmooth the images. 


\subsection{Significance analysis of the mapping function}

This section evaluates the tensor-based functional in Theorem 2, chapter 4 , and the effects of the mapping function.

The main focus in this section is to give additional details and analysis of the effects of the mapping function estimated from local densities. The numerical evaluation is presented and results are analysed both quantitatively and qualitatively.

\subsubsection{Evaluation setup}

As test data we use simple shapes shown in figure 13.8 and they include a triangle, circle, square and cross in both greyscale and colour versions. To evaluate if the introduction of a mapping function into the filtering scheme is meaningful in practical cases we perform a large-scale evaluation of all methods on the Berkeley test image database 65. The test set contains 100 images that are corrupted by 10, 15, 20, 30, 40 and 50 standard deviations of additive Gaussian noise.

The CIELAB colour representation is used to perform the filtering. The oversegmentation method is computed by using superpixels extracted via energy-driven sampling (SEEDS) [30].

Furthermore, the tensor $W$ and the mapping function in the functional 4.15 is set to describe total-variation (TV) 79, total-variation with a mapping function $(\mathrm{TVm})$ from section 5.3 gradient energy tensor total variation (GETV) from Definition 2, and GETV with a mapping function (GETVm) Definition 3. Also, we denote the selection of $W$ as in (9.2) by GETm and by GET we mean $m(u)=u$.

\subsubsection{Parameter settings}

The result images were obtained by filtering until there is no improvement in the PSNR measure. The gradient energy tensor was discretised as described in section 13.2 .2 . However, in this section we only apply a Gaussian filter of standard deviation 1 on the image data before computing the gradient energy tensor. All compared methods are minimized using a forward Euler scheme with stepsize $10^{-4}$.

\subsubsection{Basic shapes}

Figure 13.8 shows some examples of oversegmentations for the used greyscale and colour shapes for 40 standard deviations of noise. Note that despite the high noiselevel the oversegmentation adheres to the structure's boundaries. The result of the greyscale denoising of basic shapes with and without a mapping function for all presented methods can be seen in figure 13.7. The bar-plot in figure 13.9 shows the PSNR values obtained for each method and shape. In figure 13.8 we show the noisy images with additive noise of 40 standard deviations in a 8 bit resolution and the corresponding oversegmentation maps.

The optimization was performed with regards to the PSNR value, and the iterative scheme was terminated when an update did not yield any improved error value. Visually all methods perform similarly. Most notably is the observation that by introducing the mapping function some noise is retained at the edges 


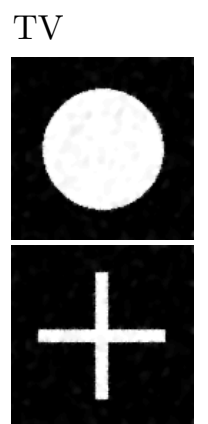

$\mathrm{TVm}$
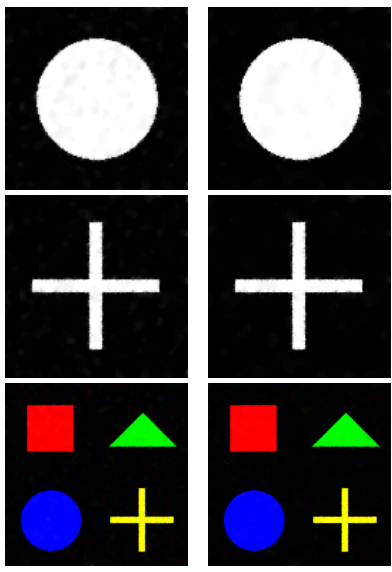

GET
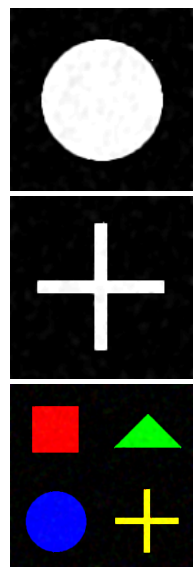

GETm
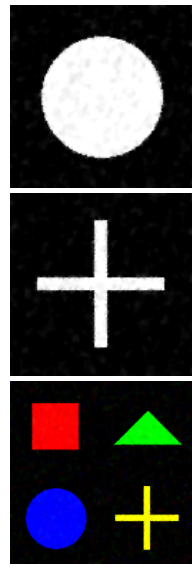

GETV

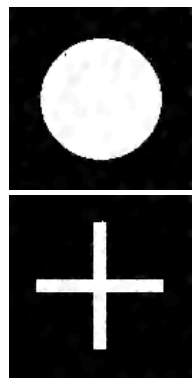

GETVm
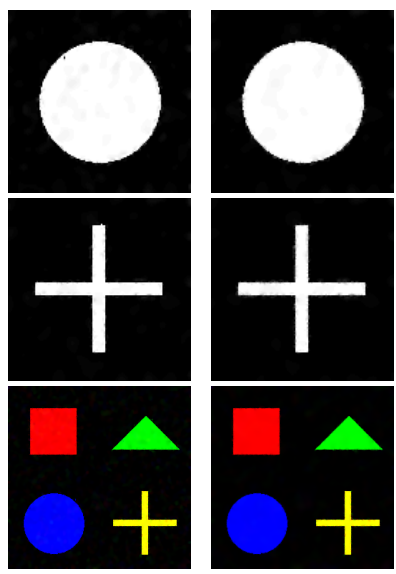

Figure 13.7: Example of colour image denoising on a synthetic image corrupted by 40 standard deviations of noise. Visually all methods performs well. Note that for TV and GETV the error measures are boosted when incorporating the mapping function

compared to not using the mapping function. This behaviour is primarily seen in figure 13.7 on the third column (GET) and the last column (GETVm), whereas the other methods does not appear to suffer from this drawback. The reason for TVm and GETVm performing well over their counterparts, in terms of the error measures, is that the enforcement of constant surfaces is more pronounced for these methods without degrading the edges and corners in the image structure. Overall the TV method benefits most from the mapping function for all shapes. The result of only using GET shows no significant improvement with regards to the PSNR-value due to the preservation of noise at edges. GETVm shows some improvement over GETV for the triangle but degrades the result on the square.

The last row of figure 13.7 shows the result of the colour image denoising where greyscale shapes have been merged to one image and been assigned different colours. Since we have access to the true noise free images we use the PSNR value and we perform the filtering in the RGB colour space. In this case the visual appearance is again similar, but the error values are improved when including the mapping function for both TV and GETV. As in the greyscale images, GET does not take advantage of the mapping function. Note that visually all methods performs well and that for TV and GETV the error measures are improved when incorporating the mapping function.

\subsubsection{Results}

Figure 13.12 shows examples of denoised results from the Berkeley images. The first 3 rows show images that have been denoised after corruption with 20 standard deviations of noise, the last two rows are examples with images that were 
corrupted by 40 standard deviations of noise. Note that the SSIM values are improved for both TV and GETV when including the oversegmentation into the diffusion scheme. In the TVm and GETVm there is some noise preserved at structure borders due to the reduction of filtering close to image borders. However, as shown in section 12.3 , the concept of driving the filtering process using an oversegmentation map significantly improved the final image quality for linear diffusion schemes.

If the scheme already describes a orientation dependent term such as the GETtensor, then the mapping function acts to further accelerate the filtering in homogeneous regions and reduces the filtering close to image borders. Thus it shows similar behaviour as the scalar valued non-linear function similar to the Perona and Malik (PM) filtering scheme [2]. But rather than controlling the flux based on the image gradient as in PM we control the flux based on the probability that an image pixel belongs to the underlying distribution. For the tensor-based methods, the difference compared to anisotropic diffusion lies in the mapping function and the used tensor. The experimental evaluation suggests that the mapping function models prior information about the denoising problem well, and helps to improve the final image quality. In the next section we perform an ANOVA significance test to verify the above observation.

\subsubsection{Error measures and confidence analysis}

Figure 13.10 shows the obtained average PSNR and SSIM values for the 100 images in the Berkeley test set compared to noise level. The evaluation has shown that methods exploiting the image statistics, modelled in the mapping function, are improved compared to not using this information. To get an accurate and objective picture of the significance of the results we performed a pairwise ANOVA one-way test where we test the null hypothesis that the group means are equal.

Figure 13.11 shows an intensity map of the obtained probability (p)-values, where a low value is indicated by dark and a high value is indicated by higher intensity white. Note that a low p-value (dark to black colour) indicates that the null hypothesis is rejected, i.e., the mean values are not the same. Also, note that the main diagonal of each noise-level is black, this is because the group-mean compared to itself is identical so we eliminated this test. As expected, at higher noise levels, the p-values increases indicating that the null hypothesis cannot be confidently rejected. Furthermore, the p-values show that GETV and GETVm are, considering the average mean values, better separated in the SSIM measure than in the PSNR. The high p-values for the two methods supports the visual appearance of the denoised result in figure 13.12 the difference between TV compared to TVm is larger than the difference between GETV and GETVm. These values show that the additional information described by the mapping function statistics, does guide methods with poor orientation information to become competitive and edge aware non-linear diffusion models. 

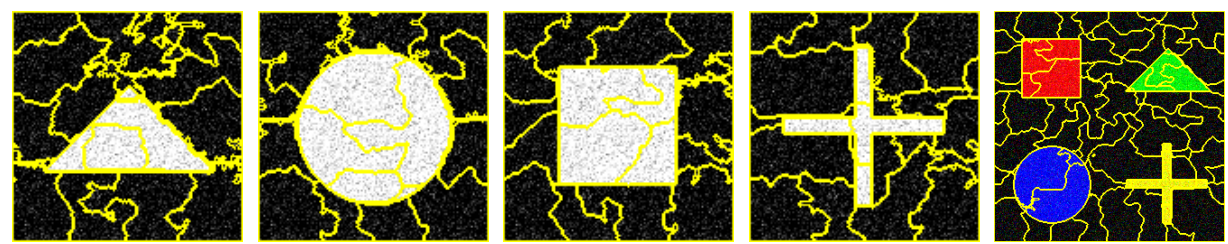

Figure 13.8: Example of images with 40 standard deviations of Gaussian noise and corresponding oversegmentation maps.

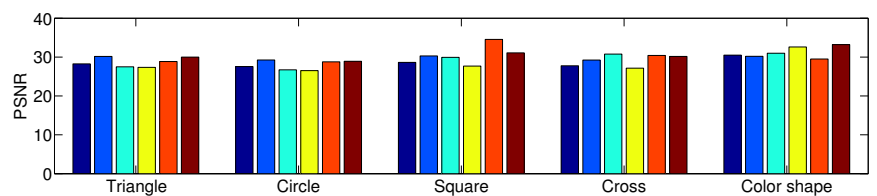

Figure 13.9: Results from greyscale basic shapes denoising.
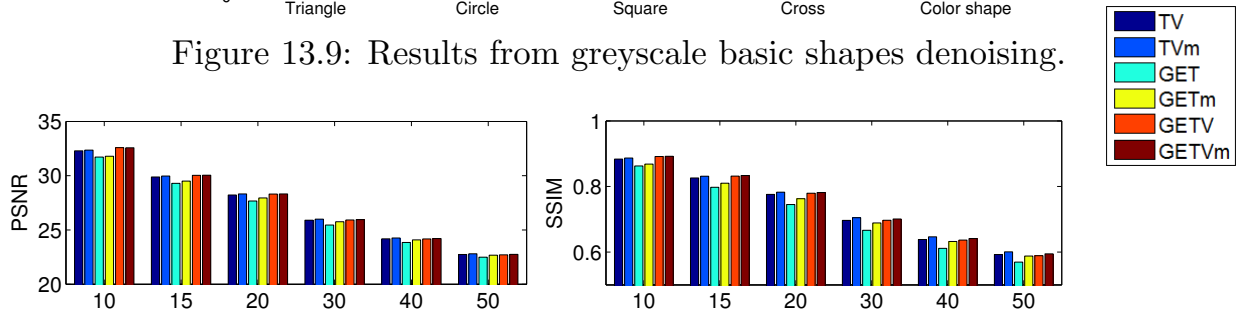

Figure 13.10: Average PSNR (left) and SSIM (right) values for the 100 images of the Berkeley test dataset. On average the introduction of the statistics from oversegmentation maps improve the final denoising quality.

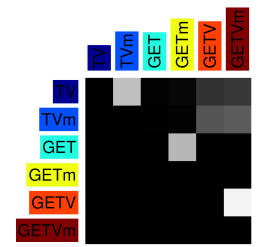

10

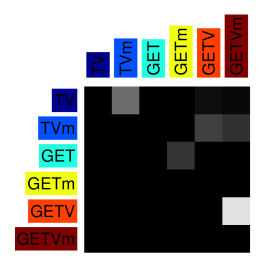

10

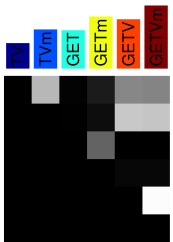

15

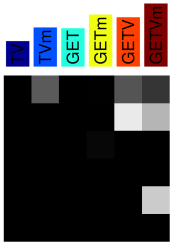

15

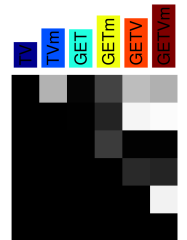

20

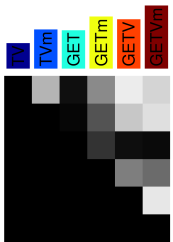

30

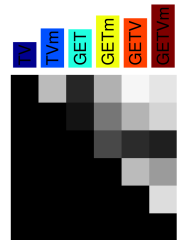

40

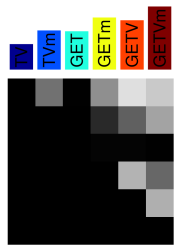

40

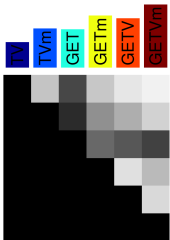

50

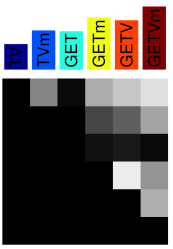

50

(b) SSIM

Figure 13.11: Pair-wise ANOVA one-way hypothesis testing on the Berkeley test colour images. The intensity indicate confirming (white) or rejecting the null hypothesis (black) that the mean-values are equal. Also note that the mapping function has a greater influence on TV than on GETV. 


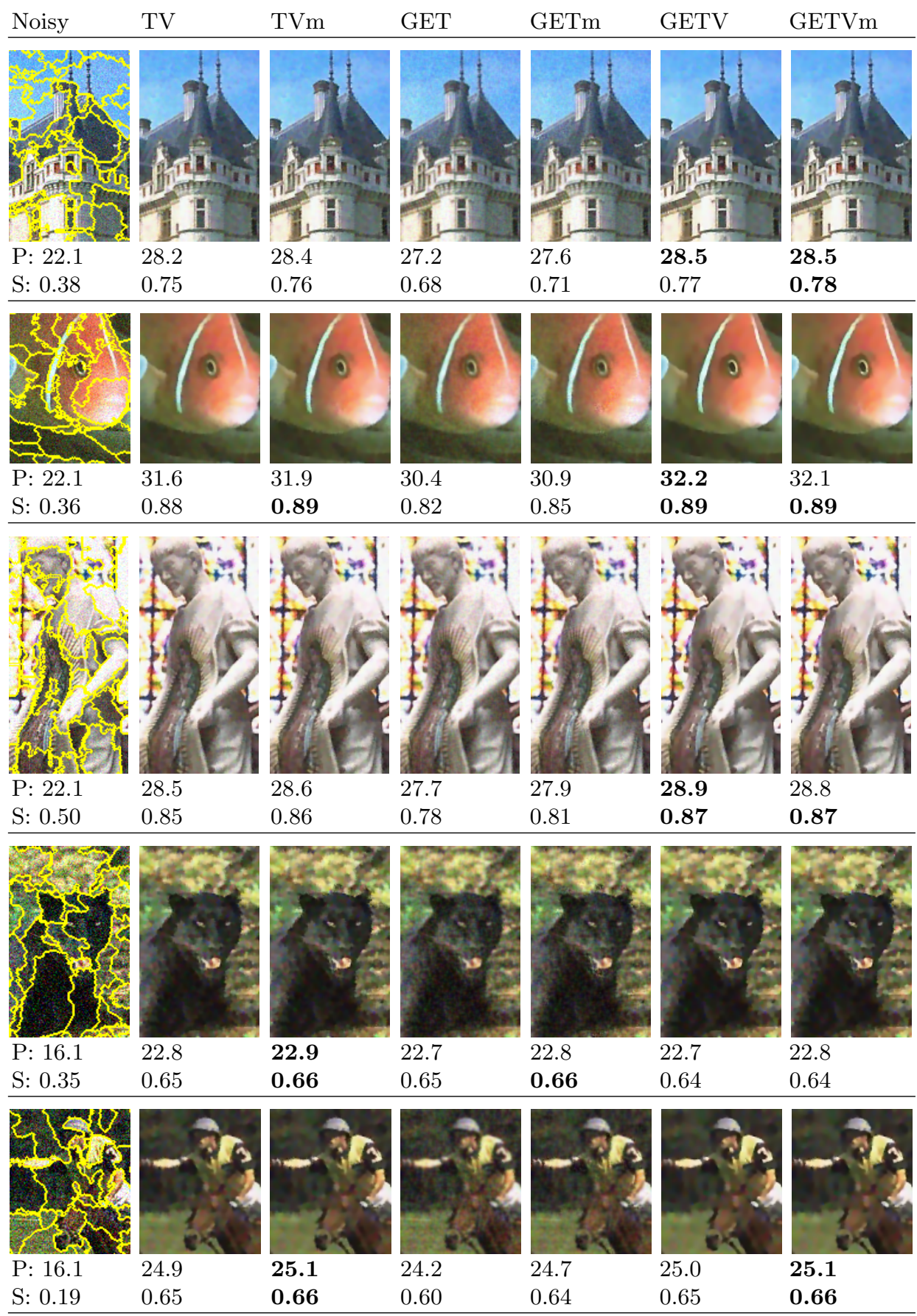

Figure 13.12: Example images from the Berkeley image database. First, second and third row were corrupted with 20 standard deviation of noise, last two rows with 40 standard deviations of noise. Note that the mapping function consistently improves the final result. $\mathrm{P}$ abbreviates PSNR and $\mathrm{S}$ abbreviates SSIM. 


\section{Fast image diffusion on the GPU}

Image enhancement methods based on tensor-based PDEs are often considered to be too computationally demanding for practical applications. The main bulk of computations is the iterative update scheme and the calculation of the postconvolution of the structure tensor components [95].

This section is an adaptation of our work [4] and the main contribution in this chapter is a novel PDE-based denoising scheme which utilise the gradient energy tensor (see chapter 8) to drive an image enhancement process. The approach is based on the classical formulation of tensor-based anisotropic diffusion and replaces the structure tensor with the gradient energy tensor. The locality of the gradient energy tensor allows for efficient use of the graphical processing unit (GPU) architecture to enable real-time image enhancement.

Before presenting the contribution, the below section introduces the main concepts of the GPU architectures.

\subsection{Introduction to GPU computing}

A graphical processing unit (GPU) is a high-performance device, often attached to a graphics card, and is designed for parallel processing of data. The GPU architecture is most suitable for problems of high spatial locality and is therefore very useful for the solution of PDEs. A GPU implementation is about how to efficiently utilise the parallelism of the GPU architecture. Using the compute unified device architecture (CUDA) framework, the parallelism is achieved by dividing the image data into blocks. Each block contains the threads that are to be executed in parallel on a streaming multiprocessor.

In an image processing setting, each pixel of an image is assigned one thread. Threads are bundled into separate blocks, and when processed by a streaming multiprocessor, each thread within a block is executed in parallel. Functions executed on the GPU are known as kernels, this means that each thread is executed within one kernel. Each pixel in the image has a unique identifier obtained by indexing 
the corresponding block (blockIdx) and offset (threadIdx) in the data, blockDim denotes a multi-vector index. An example is

int $\mathrm{x}=$ blockIdx. $\mathrm{x} *$ blockDim $. \mathrm{x}+$ threadIdx. $\mathrm{x}$;

For memory access efficiency reasons it is beneficial to use block sizes defined as multiples of 32, also known as a warp. A commonly used block size is, e.g., 256 which can be a two-dimensional spatial region of $16 \times 16$ pixels. If the image data is spatially aligned, one warp that is read from the memory is coalesced, meaning that the time required for memory retrieval is minimized.

The first step to process the image data on the GPU is to transfer the image to the GPU global memory. The global memory is the GPUs largest memory bank. The memory is suitable for holding the image data while being processed by the streaming multiprocessors, however, it is also the slowest memory type. Therefore, image operations involving the global memory should be minimized. Depending on the image processing application, different types of memories are available in the GPU. For example, the texture memory is a global read-only memory and enables hardware support for interpolation. In many cases, such as convolution operations, it is beneficial to use the shared memory that is on-chip [74]. The drawback is that the shared memory is only defined per-block. Other memory types that are frequently used include the constant memory and registers.

The following sections present the adaptive tensor-based image denoising formulation and the implementation details of the proposed scheme.

\subsection{Image diffusion on the GPU}

The structure tensor introduced by Förstner and Gülch [39] and Bigün and Granlund [16] is an integral part of many image processing applications, such as corner detection [46, 83], optical flow [64] and tensor-based image denoising [95].

It is here proposed to replace the structure tensor with an alternative tensor, the gradient energy tensor [37] that does not (necessarily) require a postconvolution of its tensor-components to form a rank-2 tensor. The principal difference to the structure tensor is that the gradient energy tensor use higher-order derivatives to capture the orientation in a neighbourhood. Empirical results show that the formulation is superior to those based on the structure tensor with regards to computational performance, even without loss of image quality.

To illustrate the computation requirement in video denoising between the two tensors, assume that the target processing speed is 24 frames per second (fps). Then the time-constraint is $1000 / 24=41.7 \mathrm{~ms}$ to process each image. As shown in table 14.1, the gradient energy tensor would require almost 4 times less computation time compared to the structure tensor.

The GPU used in the experiments is Nvidia's GTX 670 with 4GB on-card memory and the workstation is equipped with an Intel Xeon CPU at $3 \mathrm{GHz}$ and 8 GB memory. Even though the hardware specification is in the middle segment of the consumer-market we show that by using the gradient energy tensor, the proposed iterative tensor-based PDE denoising scheme can reach near maximum PSNR at 60 frames per second (fps) for a $1280 \times 720$ three channel colour pixel 
- Required convolutions, greyscale images

\begin{tabular}{ccccc} 
& $\begin{array}{c}\text { Pre-convolutions } \\
\text { (image gradient) }\end{array}$ & $\begin{array}{c}\text { Post-convolutions } \\
\text { (tensor-components) }\end{array}$ & total & available ms/image \\
\hline ST & 1 & 3 & 4 & 10.4 \\
GET & 1 & 0 & 1 & 41.7
\end{tabular}

- Required convolutions, colour images

\begin{tabular}{ccccc} 
& $\begin{array}{c}\text { Pre-convolutions } \\
\text { (image gradient) }\end{array}$ & $\begin{array}{c}\text { Post-convolutions } \\
\text { (tensor-components) }\end{array}$ & total & available ms/image \\
\hline ST & 3 & 9 & 12 & 1.2 \\
GET & 3 & 0 & 3 & 4.6
\end{tabular}

Table 14.1: Table describing the computation times for the gradient energy tensor compared to the structure tensor with regards to required convolutions. Note that the computation time for the gradient energy tensor is almost 4 times lower than the structure tensors due to fewer convolutions.

image (HD720p). This is a significant improvement over the structure tensor running at $30 \mathrm{fps}$ while achieving similar peak signal-to-noise ratio (PSNR) and structural similarity [19] (SSIM) values.

\subsubsection{Filtering scheme}

The standard formulation of tensor-based anisotropic diffusion 95 using the structure tensor is restated in the PDE below, with natural boundary conditions

$$
\left\{\begin{array}{rl}
u-u_{0}-\lambda \operatorname{div}(D(\nabla u) \nabla u) & =0 \text { in } \Omega \\
n \cdot \nabla u & =0 \text { on } \partial \Omega
\end{array},\right.
$$

where $\lambda$ is a stepsize parameter which controls the smoothness of the solution $u$ that minimizes the PDE. In 14.1), $D(\nabla u)$ is the diffusion tensor computed as

$$
D(T(\nabla u))=\Psi\left(\mu_{1}\right) v v^{t}+\Psi\left(\mu_{2}\right) w w^{t}
$$

where $v, w$ and $\mu_{1,2}$ are the eigenvectors and eigenvalues of the structure tensor $T$ in 2.34). The diffusivity function, $\Psi$, is set as $\Psi(s)=\exp (-s / k)$ where $k$ is the edge-stopping parameter determining the adaptivity to the image structure. Instead of using the diffusion tensor in the PDE (14.1) we propose to use the gradient energy tensor with positive eigenvalues, $G E T^{+}$in $(8.7)$, as the tensor controlling the orientation estimate of the image structures, i.e., we define the following PDE

$$
\left\{\begin{array}{rl}
u-u_{0}-\lambda \operatorname{div}\left(D^{+}(\nabla u) \nabla u\right) & =0 \text { in } \Omega \\
n \cdot \nabla u & =0 \text { on } \partial \Omega
\end{array} .\right.
$$




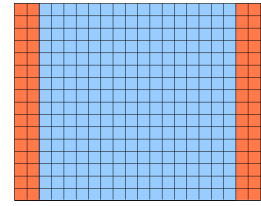

(a) Convolution rows

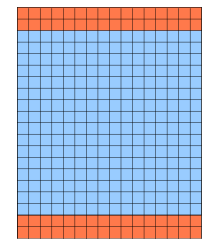

(b) Convolution columns

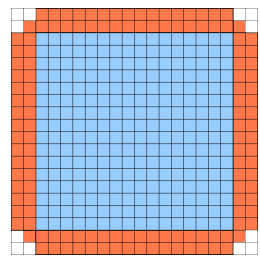

(c) Gradient energy tensor

Figure 14.1: Tiles of size $16 \times 16$ (blue regions) with padding of 2 pixels (red region) for the convolution and computation of the gradient energy tensor. We use corresponding tile layouts for computing the image gradient and filter update.

The $G E T^{+}$is transformed to align parallel to the image structure according 2.25, i.e.

$$
D^{+}\left(G E T^{+}(\nabla u)\right)=\left(\frac{\Psi\left(\lambda_{1}\right)-\Psi\left(\lambda_{2}\right)}{\lambda_{1}-\lambda_{2}}\right)\left(G E T^{+}-\lambda_{2} I\right)+\Psi\left(\lambda_{2}\right) I
$$

where $\lambda_{1,2}$ are the eigenvalues of $G E T^{+}$and we set $S=G E T^{+}$. In practice we compute (14.2) using (14.4) with $S=T$.

In order to solve the PDEs 14.1 and 14.3, we use a forward Euler scheme with finite differences to approximate the image derivatives. For a discussion on the numerical stability of the iterative scheme see chapter 10.

\subsection{Implementation details}

In this section we implement the proposed iterative denoising scheme on the GPU. We show how to utilise the highly parallelizable nature of iterative PDE-based denoising schemes and benefit from the locality of the gradient energy tensor. The implementation is done using CUDA programming language with OpenGL support.

The implementation is focused on utilising the high-performance shared memory. We do this by coalescing memory access when streaming data from global memory to shared memory. It was considered to use the texture memory due to its automatic handling of Neumann boundary conditions and memory-access optimisation for localized reads. However, texture memory is read-only and we require dynamic updates of intermediate results. Also, shared memory is on-chip, and therefore read-access requires fewer clock cycles than the texture memory. These differences in memory-latency have a significant impact on runtime performance, for example in convolutions [74].

Since we are interested in processing images with three colour channels, the implementation is simplified by defining a container describing the three colour channels red, green and blue as well as the alpha channel (required for visualization using OpenGL). The image data is read and written using 24 bit but during the filter process we use single-precision float values. There are primarily three steps involved in the iterative filtering scheme: pre-filtering for regularization of 
Anisotropic diffusion [95]

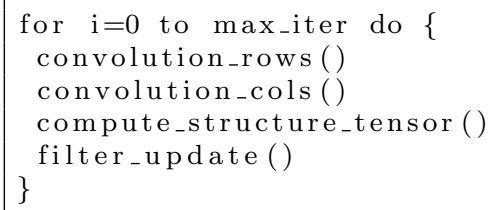

Table 14.2: Algorithm pseudo-code for the main CUDA kernels. Left: Standard approach to adaptive image diffusion using the structure tensor. Right: adaptive image diffusion using the gradient energy tensor. The convolutions are separable Gaussian functions of size $5 \times 5$ with standard deviation of 1 .

the image derivatives, orientation estimation and filter update. The steps are illustrated in table 14.2 and highlights the primary difference between the two implementations: the computation of the structure tensor requires three full (separable) convolutions of the image data whereas the energy tensor does not require any post-convolution of the tensor-components, which is key to the gain in computation speed. Figure 14.1 shows the memory layout of the shared memory that we use in the CUDA kernels shown in table 14.2. We use tile sizes of $16 \times 16$ and note that each entry in the tile consists of four entries, i.e., red, green, blue and alpha channels. This approach is convenient since it both simplifies the code and facilitates efficient memory access. The padding of the tiles (the red region in figure 14.1 is done with two pixels in the case of the separable convolution since we use a Gaussian filter of size $5 \times 5$ with standard deviation of 1 for smoothing the image and tensor-components. The coefficients of the Gaussian filter are set in the constant memory. The shared memory associated with the gradient energy tensor require a padding of 2 pixels in horizontal and vertical direction since the support of the third order finite difference derivative is 5 pixels. Lastly, since we only require first order diagonal derivatives, it is sufficient to read the closest corner-pixel into the shared memory, further simplifying the global memory access pattern.

The resulting computation times for each filter and image size is measured by using the standard sdkStartTimer() and sdkStopTimer() available in CUDA. The OpenGL rendering is included in the timing of the filter performance as shown in table 14.3 since it more accurately reflects the expected real-time capabilities of 


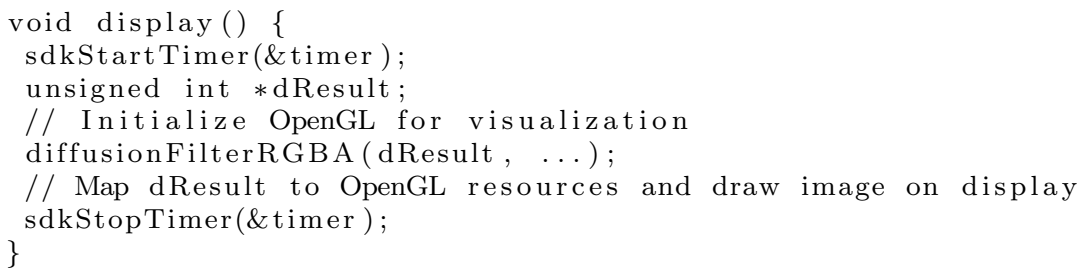

Table 14.3: Main function of the tensor-based image denoising method. The timer values reported in section 14.4 are timed including the OpenGL rendering.

video denoising where each frame in a video sequence would be visualized.

\subsection{Results}

The focus of our evaluation is to show that the gradient energy tensor does not compromise the denoising quality compared to the structure tensor while achieving a faster runtime. The measures include the peak signal-to-noise ratio (PSNR) and the structure similarity index 94] (SSIM) for the image quality.

Figure 14.3 shows the colour test image pippin_Florida0002.bmp from the McGill colour image database [71] with the original resolution $2560 \times 1920$ pixels. The image was downsampled (using bicubic interpolation) to $1920 \times 1080$ (HD1080) and $1280 \times 720($ HD720), two common image resolutions in high-definition (HD) video. Each image is corrupted with 20 standard deviations of normal distributed noise. The filtering scheme is iterated 10 times with a fixed update step of size 0.20 . The diffusivity constant (see $\Psi$ in (14.4) ) is set to $k / 10$ where $k=$ $\left(e^{1}-1\right) /\left(e^{1}-2\right) \sigma^{2}[33$, this yields $k / 10=0.0015$ when $\sigma=20 / 255$ and each colour channel is quantized using an 8 bit representation.

Figure 14.2 shows the obtained PSNR (a) and SSIM (b) values compared to the iteration number, as expected the two methods are comparable for the obtained error measures. The best error values are in agreement between the two methods but obtained at different iterations numbers, it is not a discrepancy in method performance but an issue of parameter tuning. In figure 14.2 (c) and (d) we show the fps that we obtain for each iteration. After four iterations, in (c), the filter using the gradient energy tensor is stable at $60 \mathrm{fps}$ whereas the standard diffusion scheme using the structure tensor has dropped to $30 \mathrm{fps}$ for the smallest image resolution. In $(d)$ we show the tradeoff between PSNR and obtained fps for both tensors: a higher fps result in a lower PSNR value. Note that the fps count is independent of the image content. Future work will include a more comprehensive study of the GET orientation estimation compared to the structure tensor for other noise levels and image types than considered here.

Figure 14.4 illustrates an example of the final denoised result, the zoomed images were cropped from the $2560 \times 1920$ resolution image and the original image was corrupted by 20 standard deviations of normal distributed additive noise. The images illustrate the denoised result after 10 iterations and note that the 


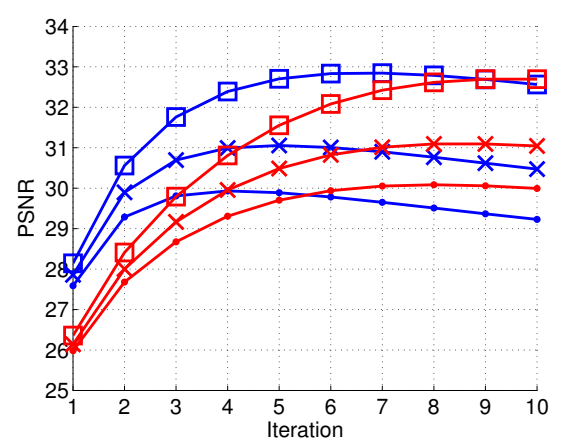

(a)

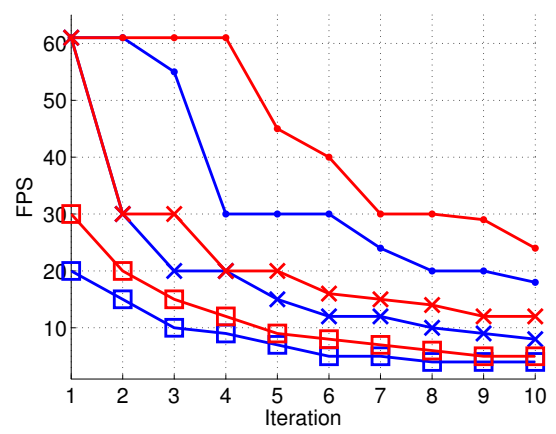

(c)

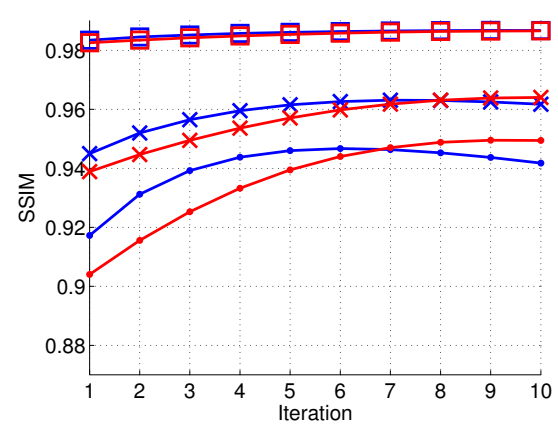

(b)

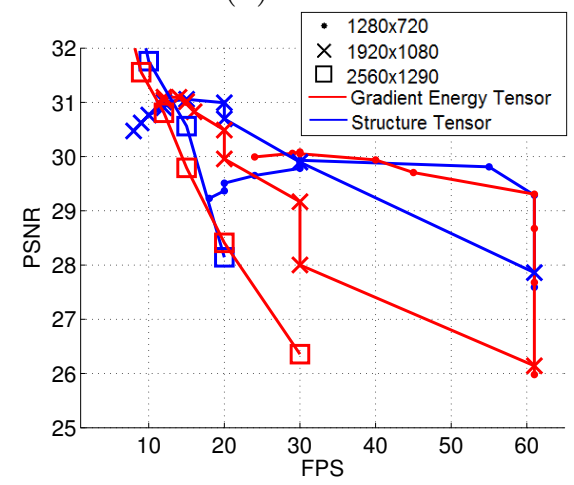

(d)

Figure 14.2: Obtained PSNR (a), SSIM (b) and (c) fps compared to iterations, and PSNR compared to fps (d) for the considered image sizes. The PSNR and SSIM values are similar for the two tensors but the obtained fps is significantly higher for the gradient energy tensor.

gradient energy tensor fps-count is $25 \%$ higher than the structure tensor but with indiscernible image quality and PSNR (cmp. figure 14.2 (a) and (c)). Table 14.4 displays the ratio of the gradient energy tensor error measures over the structure tensor error measures averaged over iterations, i.e., if the ratio is 1.0 there is no difference in method performance, if the value is larger than 1.0 then the ratio indicate that the gradient energy tensor performs better. The SSIM value difference is less than $10^{-3}$ whereas the PSNR shows a $3.2 \%$ difference in the favour of the structure tensor, however considering the final fps-count the gradient energy tensor is up to $40 \%$ more computationally efficient for the largest image size.

Thus, GET does show a comparable performance to the structure tensor with respect to local orientation estimation as was shown in chapter 8 . This lead us to use the GET instead of the structure tensor for image filtering and by utilising the structure of the GPU architecture we are able to outperform the structure tensor formulation with regard to run-time while achieving comparable image quality 

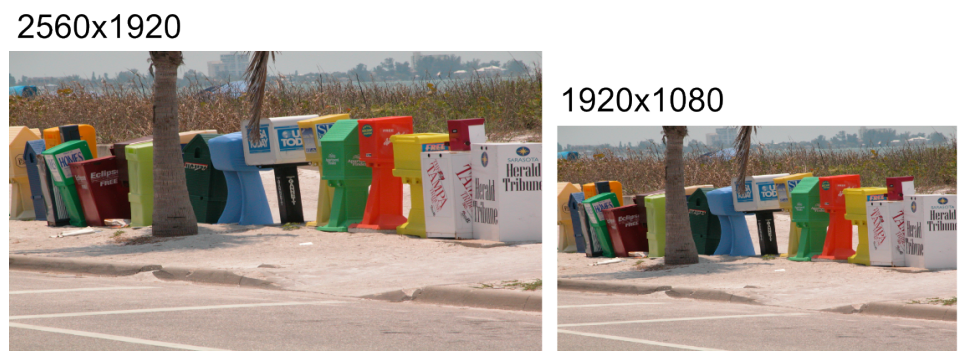

$1280 \times 720$

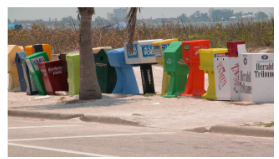

Figure 14.3: Colour test images and the corresponding image sizes in pixels that are used in the evaluation.
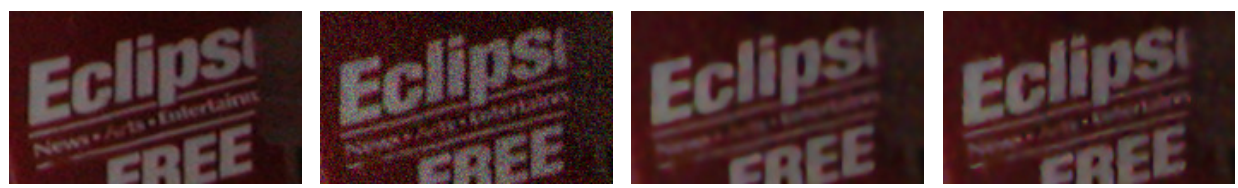

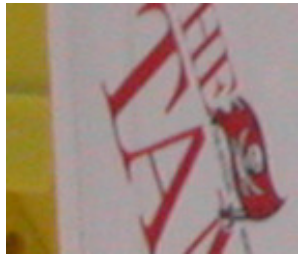

Original

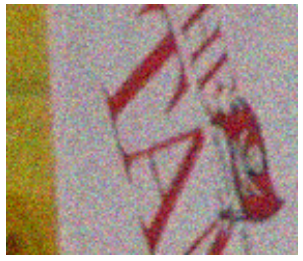

Noisy

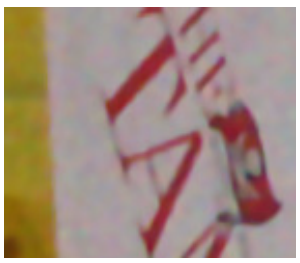

Structure tensor

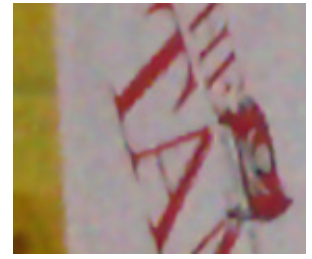

Energy Tensor

Figure 14.4: Two patches from the $2560 \times 1920$ resolution image and the corresponding denoised images at iteration 10. The applied noise was 20 standard deviation of normal distributed additive noise. Note that the difference in visual appearance is not discernible but the fps count is improved by $25 \%$.

\begin{tabular}{c|ccc} 
Ratio & PSNR & SSIM & fps \\
\hline $1280 \times 720$ & 0.991 & 0.995 & 1.35 \\
$1920 \times 1080$ & 0.982 & 0.997 & 1.27 \\
$2560 \times 1920$ & 0.968 & 0.999 & 1.40
\end{tabular}

Table 14.4: Ratios are computed as the measure obtained by the gradient energy tensor divided by the measure obtained by the structure tensor and averaged over iterations. A ratio of 1.0 indicates that the performance is identical. The SSIM and PSNR ratios are nearly identical but at up to $40 \%$ higher fps values in the largest image resolution.

measures. A drawback of the presented evaluation is that we only considered achieved frames per second in relation to the obtained PSNR and SSIM values. Since it is well known that error measures do not always correlate with perceived image quality it would be of interest to perform an extended evaluation. 


\section{Chapter 15}

\section{Conclusions}

This chapter summarises the results presented in this thesis, conclusions and possible directions of future research. Evaluation and the main results of all methods presented in this thesis are described in part IV.

\subsection{Summary of the results}

The motivation of this thesis was to derive and study the connection between the existence of a variational formulation, tensor-based regularization and contextual information. In this regard, the thesis introduced and formulated a tensor-based regularization term in Theorem 2. Theoretical and practical aspects are studied for several applications, specifically in the context of visualization and denoising of computed tomography data, and diffusion using probability density functions. The variational formulation of the tensor-based non-linear domain formulation described in Theorem 2 is a general framework. Based on all the findings presented in this thesis I conclude the following:

- It is possible to model domain-specific information in a tensor-based variational framework.

The strength of the model lies in the domain-specific knowledge that is incorporated into the framework by using the non-linear "mapping function".

Throughout this work, the tensor-based framework in Theorem 2 has been adapted to form novel diffusion schemes. One of the most successful formulations was to incorporate local density estimates into the diffusion process in chapter 6 These estimates are derived from a structure-preserving oversegmentation of the input image. The oversegmentation results in a number of homogeneous, edgealigned segments, within which, density functions can be easily and independently estimated. The estimated densities drive the diffusion process such that it adheres to image boundaries resulting in a non-linear image enhancement scheme, comparable to state-of-the-art denoising methods. 
In chapter 4 it is proven that there exists a connection between the main tensorbased functional and the corresponding Euler-Lagrange equation. We showed properties of the resulting scheme with regards to symmetry constraints and convexity. Based on the extensive evaluations in chapters 12 and 13 , and in particularly section 13.3 it has been shown that the mapping function does improve the accuracy of the filtering schemes. Therefore, I believe that the ideas presented in this thesis can be exploited in related problem domains where additional knowledge of the image scene is available, e.g., in multi-sensor systems.

The introduction of orientation information into the functional described by tensor-valued functions and in conjunction with the domain-dependent function, is the most important contribution of this thesis. Currently, the mapping function is a global or semi-local function. However, by further relaxing the definition of the mapping function, it can be extended to enable users to interact with the filtering scheme to target the filter to image regions which are of particular interest, such as objects or surfaces.

A classical problem, considered in section 4.4, is to find a general theory that formulates conditions for a variational formulation to exist given a tensor-based PDE. We succeeded for the case of rank 1 tensors when the signal has an intrinsically one-dimensional structure (such as lines) in Theorem 3 . In Corollary 4. we explicitly state why no variational formulation can exist to a PDE formulated with the structure tensor.

Additionally, chapter 7 considered diffusion methods expressed in the channel representation framework. The aim was to introduce a robust estimator of the image signal into the framework of non-linear diffusion to handle the case of impulse noise. It turned out that including the channel framework into the regularization leads to a robust filtering well suited for images corrupted with Gaussian as well as impulse noise. By expressing the image data using B-spline basis functions, we derived a diffusion framework that oversmooths outliers in the image data. The approach can be interpreted in terms of channel smoothing: rather than performing Gaussian filtering in separate channels, the channel-based regularization controls the diffusivity based on the channel decomposition. We analysed the denoising behaviour of the proposed method on commonly used scalar valued images and compared the methods to similar as well as state-of-the-art methods.

We note that the opponent colour transform 11.1 - 11.3 is not unique and we are aware that a plethora of colour transformations exists which probably would work equally well as the one considered. For example, in sections 13.2 and 13.3 we used the CIELAB colour transform which yields visually plausible results. However, the transformation defined by (11.1)- 11.3 is derived from a theoretical point of view, not from a perceptual description of the colour space, i.e., the considered transformation yields a much simpler representation of the opponent colour space and thus is more suitable for theoretical applications.

With regards to the numerical schemes described in chapter 10 , it has been satisfactory to achieve discrete approximations of the PDEs that work sufficiently well with respect to numerical stability. However, it would be interesting to compare the performance of the proposed methods in the case of more accurate numerical schemes. The current solution method (forward Euler scheme) has the limitations 
of stability and the size of the time-update step. Particularly, the gradient energy total variation scheme in chapter 9 would benefit from a more appropriate discretisation scheme.

\subsection{Future work}

The following paragraphs highlight some directions of future work that are natural continuations of the methods presented in this thesis.

In chapter 4.2 we derived the transformation of the image statistics under the influence the non-linear mapping function. It was found that the energy operator is involved in the variance transformation. This is not a track that, at the time, was pursued. However, it would be interesting to further study the noise transformation and further adopt the statistics into Theorem 2 .

Currently, the diffusion framework (in particularly density driven diffusion in chapter 6) and image statistics are decoupled as two separate processes. The extension of this line of research is to join these two aspects into one coherent framework. This direction can enable unexpected results where statistics meets variational tensor-based diffusion methods. The difficulty lies in the modelling of the underlying stochastic process and how to couple it with locally homogeneous regions. In particular, an open problem is how to modify the image data value distribution while updating the region boundaries to simultaneously achieve an edge preserving denoising effect.

The gradient energy tensor has been successfully used as a replacement for the structure tensor in corner detection, optical flow and anisotropic diffusion. The tensor has not been used to explicitly formalize these applications, which would be the natural continuation for further studies.

The concept of a mapping function and the infusion of domain-dependent information into the filtering schemes has proven to be, both theoretically and practically, very successful. One line of future work to pursue is to consider other problem domains as well in addition to the case of image denoising. Moreover, due to the general definition of the mapping function, it can in principle, model (almost) any type of additional constraint. Such constraints can include, e.g., information collected from image databases, other types of image representations than the channel representation, or the image geometry.

An extension of the GPU formulation in chapter 14 is to consider video. However, an issue which needs addressing is that video sequences also exhibit a high temporal correlation and thus contain noise also in the temporal domain in addition to spatial noise. Approaches to deal with temporal noise can include causal formulations which would process one frame or a pair of frames in a recursive manner. Non-causal systems would process batches of frames, achieving good noise suppression while suffer from a delayed video playback if processing live video.

Finally, injecting domain-dependent information into the problem formulation is a promising direction of continued research. I believe that the future lies in context aware operators that can be motivated from theoretic and practical standpoints in contrast to "blind" image filtering methods. 


\section{Appendix A}

\section{Appendices}

\section{A.1 Proof Theorem 2}

Proof. To prove Theorem 2, compute the variational derivative of the functional (4.15). The first variation is given by

$$
\partial_{u} R(u) v=\left.\frac{\partial}{\partial \varepsilon} R(u+\varepsilon v)\right|_{\varepsilon=0},
$$

where

$$
R(u)=\int_{\Omega} \nabla m(u) W(\nabla m(u)) \nabla m(u) d x .
$$

Now, expand $R(u+\varepsilon v)$

$$
\begin{aligned}
R(u+\varepsilon v) & =\nabla^{t} m(u+\varepsilon v) W(\nabla m(u+\varepsilon v)) \nabla m(u+\varepsilon v) \\
& =\underbrace{m^{\prime}(u+\varepsilon v)^{2} \nabla^{t}(u+\varepsilon v)}_{=A} \underbrace{W(\nabla m(u+\varepsilon v)) \nabla(u+\varepsilon v)}_{=B},
\end{aligned}
$$

then

$$
\frac{\partial}{\partial \varepsilon} R(u+\varepsilon v)=\left(\frac{\partial}{\partial \varepsilon} A(u+\varepsilon v)\right) B+A\left(\frac{\partial}{\partial \varepsilon} B(u+\varepsilon v)\right)
$$

Define

$$
z=\nabla m(u+\varepsilon v)=\left(\begin{array}{c}
z_{1}=m^{\prime}(u+\varepsilon v)\left(u_{1}+\varepsilon v_{1}\right) \\
\vdots \\
z_{n}=m^{\prime}(u+\varepsilon v)\left(u_{n}+\varepsilon v_{n}\right)
\end{array}\right),
$$

and note that

$$
\left.z\right|_{\varepsilon=0}=\nabla m(u)=s .
$$

In order to differentiate the $B$-component use the differentiation rule

$$
\frac{\partial W(z)}{\partial \varepsilon}=\frac{\partial W(z)}{\partial z_{1}} \frac{\partial z_{1}}{\partial \varepsilon}+\cdots+\frac{\partial W(z)}{\partial z_{n}} \frac{\partial z_{n}}{\partial \varepsilon},
$$


then

$$
\begin{aligned}
\frac{\partial}{\partial \varepsilon} B(z)= & \frac{\partial}{\partial \varepsilon}(W(z) \nabla(u+\varepsilon v)) \\
= & \left(\frac{\partial}{\partial \varepsilon} W(z)\right) \nabla(u+\varepsilon v)+W(z) \frac{\partial}{\partial \varepsilon} \nabla(u+\varepsilon v) \\
= & {\left[W_{z_{1}}(z)\left(m^{\prime \prime}(u+\varepsilon v) v\left(u_{1}+\varepsilon v_{1}\right)+m^{\prime}(u+\varepsilon v) v_{1}\right)\right.} \\
& \vdots \\
& \left.+W_{z_{n}}(z)\left(m^{\prime \prime}(u+\varepsilon v) v\left(u_{n}+\varepsilon v_{n}\right)+m^{\prime}(u+\varepsilon v) v_{n}\right)\right] \nabla(u+\varepsilon v), \\
& +W(z) \nabla v
\end{aligned}
$$

and

$$
\begin{aligned}
&\left.\frac{\partial}{\partial \varepsilon} B(z)\right|_{\varepsilon=0}= {\left[W_{s_{1}}(s)\left(m^{\prime \prime}(u) v u_{1}+m^{\prime}(u) v_{1}\right)\right.} \\
& \vdots \\
&+ \\
&\left.+W_{s_{n}}(s)\left(m^{\prime \prime}(u) v u_{n}+m^{\prime}(u) v_{n}\right)\right] \nabla u \\
&+W(s) \nabla v
\end{aligned}
$$

then the second product in A.4 reads

$$
\begin{gathered}
\left.A\left(\frac{\partial}{\partial \varepsilon} B(u+\varepsilon v)\right)\right|_{\varepsilon=0}=m^{\prime}(u)^{2} \nabla^{t} u\left[W_{s_{1}}(s)\left(m^{\prime \prime}(u) v u_{1}+m^{\prime}(u) v_{1}\right)\right. \\
\vdots \\
\left.+W_{s_{n}}(s)\left(m^{\prime \prime}(u) v u_{n}+m^{\prime}(u) v_{n}\right)\right] \nabla u \\
+m^{\prime}(u)^{2} \nabla^{t} u W(s) \nabla v \\
=m^{\prime}(u)^{2}\left[\nabla^{t} u W_{s_{1}}(s) \nabla u\left(m^{\prime \prime}(u) v u_{1}+m^{\prime}(u) v_{1}\right)\right. \\
\vdots \\
\left.+\nabla^{t} u W_{s_{n}}(s) \nabla u\left(m^{\prime \prime}(u) v u_{n}+m^{\prime}(u) v_{n}\right)\right] \\
+m^{\prime} \nabla^{t} u W(s) \nabla v .
\end{gathered}
$$

To simplify notation, define

$$
Q=\left(\begin{array}{c}
\nabla^{t} u W_{s_{1}}(s) \nabla u \\
\vdots \\
\nabla^{t} u W_{s_{n}}(s) \nabla u
\end{array}\right)=\left(\begin{array}{c}
\nabla^{t} u W_{s_{1}}(s) \\
\vdots \\
\nabla^{t} u W_{s_{n}}(s)
\end{array}\right) \nabla u={ }_{\nabla u} W_{s}(s) \nabla u .
$$


then

$$
\begin{aligned}
& A .9=m^{\prime}(u)^{2}\left\langle Q,\left(m^{\prime \prime}(u) v \nabla u+m^{\prime}(u) \nabla v\right)\right\rangle \\
& +m^{\prime}(u)^{2}\langle\nabla u, W(s) \nabla v\rangle \\
& =m^{\prime}(u)^{2} m^{\prime \prime}(u)\langle Q, \nabla u\rangle v+m^{\prime}(u)^{3}\langle\nabla v, Q\rangle \\
& +m^{\prime}(u)^{2}\left\langle\nabla v, W^{t}(s) \nabla u\right\rangle \\
& =\underbrace{\left\langle m^{\prime}(u)^{2} m^{\prime \prime}(u) Q, \nabla u\right\rangle}_{=G} v \\
& +\left\langle\nabla v,(\underbrace{m^{\prime}(u)^{3} Q+m^{\prime}(u)^{2} W^{t}(s)}_{=H}) \nabla u\right\rangle \\
& =G v+\langle\nabla v, H \nabla u\rangle,
\end{aligned}
$$

Now consider the first product of A.4. The derivative of the $A$-component in A.3

$$
\begin{aligned}
\frac{\partial}{\partial \varepsilon} A(u+\varepsilon v) & =\frac{\partial}{\partial \varepsilon} m^{\prime}(u+\varepsilon v)^{2} \nabla^{t}(u+\varepsilon v) \\
& =2 m^{\prime}(u+\varepsilon v) m^{\prime \prime}(u+\varepsilon v) v \nabla^{t}(u+\varepsilon v)+m^{\prime}(u+\varepsilon v)^{2} \nabla^{t} v
\end{aligned}
$$

which result in the the product

$$
\begin{gathered}
\left.\left(\frac{\partial}{\partial \varepsilon} A(u+\varepsilon v)\right) B\right|_{\varepsilon=0}=\left(2 m^{\prime}(u) m^{\prime \prime}(u) v \nabla^{t} u+m^{\prime}(u)^{2} \nabla^{t} v\right) W(\nabla m(u)) \nabla u \\
=(\underbrace{2 m^{\prime}(u) m^{\prime \prime}(u) \nabla^{t} u W(\nabla m(u)) \nabla u}_{E}) v \\
\quad+\nabla^{t} v(\underbrace{m^{\prime}(u)^{2} W(\nabla m(u))}_{F}) \nabla u \\
=E v+\nabla^{t} v F \nabla u .
\end{gathered}
$$

Adding A.12 and A.14 yield

$$
\delta R=\left.\frac{\partial}{\partial \varepsilon} R(u+\varepsilon v)\right|_{\varepsilon=0}=(G+E) v+\nabla^{t} v(H+F) \nabla u,
$$

and by applying Green's first identity on the $\nabla v$ term

$$
\begin{aligned}
\int_{\Omega} \nabla^{t} v(H+F) \nabla u d x= & \int_{\partial \Omega} v(n \cdot(H+F) \nabla u) d S \\
& -\int_{\Omega} v \operatorname{div}((H+F) \nabla u) d x
\end{aligned}
$$


we obtain the final Euler-Lagrange equation

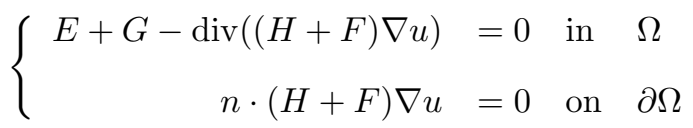

collecting the terms results in

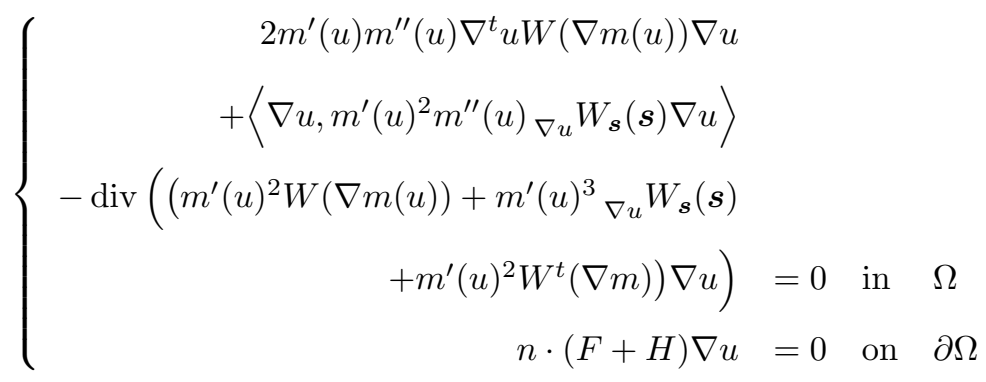

dropping the parenthesizes and rearranging the E-L equation yield

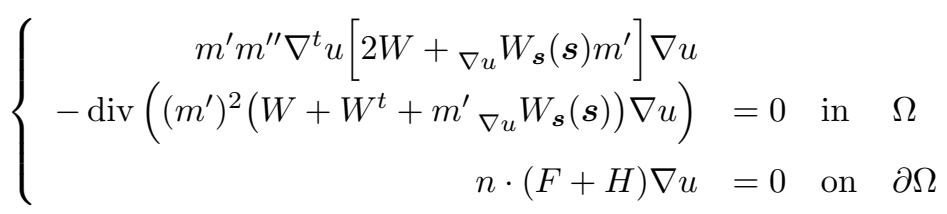

It is possible to simplify the above E-L equation further. Consider the expansion

$$
\begin{aligned}
\operatorname{div}\left(\left(m^{\prime}\right)^{2}[2 W\right. & \left.\left.+{ }_{\nabla u} W_{\boldsymbol{s}}(\boldsymbol{s}) m^{\prime}\right] \nabla u\right) \\
=2 & m^{\prime} m^{\prime \prime} \nabla^{t} u\left[2 W+{ }_{\nabla u} W_{\boldsymbol{s}}(\boldsymbol{s}) m^{\prime}\right] \nabla u \\
& \quad+\left(m^{\prime}\right)^{2} \operatorname{div}\left(\left(2 W+{ }_{\nabla u} W_{\boldsymbol{s}}(\boldsymbol{s})\right) m^{\prime} \nabla u\right),
\end{aligned}
$$

and by inserting it into the PDE of the E-L equation, we get

$$
\begin{aligned}
& \operatorname{div}\left(\left(m^{\prime}\right)^{2}\left[2 W+{ }_{\nabla u} W_{\boldsymbol{s}}(\boldsymbol{s}) m^{\prime}\right] \nabla u\right) \\
& -\left(m^{\prime}\right)^{2} \operatorname{div}\left(\left[2 W+{ }_{\nabla u} W_{\boldsymbol{s}}(\boldsymbol{s}) m^{\prime}\right] \nabla u\right) \\
& \quad-2 \operatorname{div}\left(\left(m^{\prime}\right)^{2}\left[W+W^{t}+m^{\prime}{ }_{\nabla u} W_{\boldsymbol{s}}(\boldsymbol{s})\right] \nabla u\right)=0,
\end{aligned}
$$

which is reformulated using the linearity of the divergence-operator

$$
\begin{aligned}
-\left(m^{\prime}\right)^{2} \operatorname{div}( & \left.\left(2 W+{ }_{\nabla u} W_{\boldsymbol{s}}(\boldsymbol{s}) m^{\prime}\right) \nabla u\right) \\
-\operatorname{div}\left(\left(m^{\prime}\right)^{2}(\right. & 2 W+2 W^{t}+2 m^{\prime}{ }_{\nabla u} W_{\boldsymbol{s}}(\boldsymbol{s}) \\
- & {\left.\left.\left[2 W+{ }_{\nabla u} W_{\boldsymbol{s}}(\boldsymbol{s}) m^{\prime}\right]\right) \nabla u\right)=0 }
\end{aligned}
$$


giving the final E-L equation as

$$
\left\{\begin{array}{r}
-\left(m^{\prime}\right)^{2} \operatorname{div}\left(\left[2 W+{ }_{\nabla u} W_{\boldsymbol{s}}(\boldsymbol{s}) m^{\prime}\right] \nabla u\right) \\
-\operatorname{div}\left(\left(m^{\prime}\right)^{2}\left[2 W^{t}+{ }_{\nabla u} W_{\boldsymbol{s}}(\boldsymbol{s}) m^{\prime}\right] \nabla u\right)=0 \\
n \cdot(F+H) \nabla u=0 \text { in } \Omega \\
\text { on } \partial \Omega
\end{array}\right.
$$

then let

$$
\begin{aligned}
& S_{1}=2 W+{ }_{\nabla u} W_{\boldsymbol{s}}(\boldsymbol{s}) m^{\prime}, \\
& S_{2}=2 W^{t}+{ }_{\nabla u} W_{\boldsymbol{s}}(\boldsymbol{s}) m^{\prime}
\end{aligned}
$$

and

$$
B=F+H=\left(m^{\prime}\right)^{2}\left[W+W^{t}+{ }_{\nabla u} W_{\boldsymbol{s}}(\boldsymbol{s}) m^{\prime}\right],
$$

which concludes the proof.

\section{A.2 Extended proof Lemma 1}

Proof. Using the identity $\operatorname{div}(C \nabla u)=\operatorname{div}(C) \nabla u+\operatorname{tr}(C H u)$ where $H$ is the Hessian, the left hand-side of 4.26 can be modified as follows

$$
\begin{aligned}
& \left(m^{\prime}\right)^{2} \operatorname{div}(C \nabla u)+\operatorname{div}\left(\left(m^{\prime}\right)^{2} C \nabla u\right) \\
& \quad=\left(m^{\prime}\right)^{2}(\operatorname{div}(C) \nabla u+\operatorname{tr}(C H u))+\operatorname{div}\left(\left(m^{\prime}\right)^{2} C\right) \nabla u+\operatorname{tr}\left(\left(m^{\prime}\right)^{2} C H u\right) \\
& \quad=\left(\left(m^{\prime}\right)^{2} \operatorname{div}(C)+\operatorname{div}\left(\left(m^{\prime}\right)^{2} C\right)\right) \nabla u+2\left(m^{\prime}\right)^{2} \operatorname{tr}(C H u) \\
& \quad=\left(\left(m^{\prime}\right)^{2} \operatorname{div}(C)+2 m^{\prime} m^{\prime \prime} \nabla^{t} u C+\left(m^{\prime}\right)^{2} \operatorname{div}(C)\right) \nabla u+2\left(m^{\prime}\right)^{2} \operatorname{tr}(C H u) \\
& \quad=2 m^{\prime}\left[\left(m^{\prime} \operatorname{div}(C)+m^{\prime \prime} \nabla^{t} u C\right) \nabla u+m^{\prime} \operatorname{tr}(C H u)\right] \\
& \quad=2 m^{\prime}\left[\operatorname{div}\left(m^{\prime} C\right) \nabla u+m^{\prime} \operatorname{tr}(C H u)\right] \\
& \quad=2 m^{\prime} \operatorname{div}\left(m^{\prime} C \nabla u\right)
\end{aligned}
$$

which concludes the proof.

In the case $C$ defined by the identity matrix, a corresponding contraction of the divergence form can be made

$$
\begin{aligned}
\left(m^{\prime}\right)^{2} & \operatorname{div}(\nabla u)+\operatorname{div}\left(\left(m^{\prime}\right)^{2} \nabla u\right) \\
& =\left(m^{\prime}\right)^{2} \Delta u+2 m^{\prime} m^{\prime \prime}|\nabla u|^{2}+\left(m^{\prime}\right)^{2} \Delta u \\
& =2\left(m^{\prime}\right)\left(\left(m^{\prime}\right) \Delta u+m^{\prime \prime}|\nabla u|^{2}\right) \\
& =2 m^{\prime} \operatorname{div}\left(m^{\prime} \nabla u\right) .
\end{aligned}
$$




\section{A.3 Derivation GETVm}

The functional in Definition 3 is

$$
R(u)=\int_{\Omega} \nabla m(u) W(\nabla m(u)) \nabla m(u) d x .
$$

Note that $\nabla m(u)=m^{\prime}(u) \nabla u$ and $W$ defined as

$$
W(\nabla m(u))=\frac{\exp \left(-G E T^{+}(\nabla m(u)) / k^{2}\right)}{|\nabla m(u)|},
$$

where $k>0$ is the diffusivity parameter. Let

$$
E=\frac{1}{|\nabla m(u)|}
$$

and

$$
D(\nabla m(u))=\exp \left(-G E T^{+}(\nabla m(u)) / k^{2}\right),
$$

where $G E T^{+}=v v^{t}\left|\mu_{1}\right|+w w^{t}\left|\mu_{2}\right|$ is the symmetric semi-positive definite gradient energy tensor (GET). The eigenvectors $v, w$ and eigenvalues $\mu_{1,2}$ below are given by the GET tensor

$$
\begin{aligned}
G E T(\nabla m(u))=[ & \left.\nabla \nabla^{t} m(u)\right]\left[\nabla \nabla^{t} m(u)\right] \\
& -\frac{1}{2}\left(\nabla m(u)[\nabla \Delta m(u)]^{t}+[\nabla \Delta m(u)] \nabla^{t} m(u)\right) .
\end{aligned}
$$

To simplify the following derivations let $m^{\prime}(u) u_{x}=s_{1}$ and $m^{\prime}(u) u_{y}=s_{2}$, then an equivalent formulation to the components of the GET-tensor is

$$
\begin{aligned}
a= & \left(\partial_{x} s_{1}\right)^{2}+\left(\partial_{x} s_{2}\right)^{2}-s_{1}\left(\partial_{x x} s_{1}+\partial_{x y} s_{2}\right) \\
b= & \left(\partial_{x} s_{1}\right)\left(\partial_{x} s_{2}\right)+\left(\partial_{y} s_{1}\right)\left(\partial_{y} s_{2}\right) \\
& \quad-\frac{1}{2}\left(s_{1}\left(\partial_{y x} s_{1}+\partial_{y y} s_{2}\right)+s_{2}\left(\partial_{x x} s_{1}+\partial_{x y} s_{2}\right)\right) \\
& \quad\left(\partial_{y} s_{2}\right)^{2}+\left(\partial_{y} s_{1}\right)^{2}-s_{2}\left(\partial_{y x} s_{1}+\partial_{y y} s_{2}\right) .
\end{aligned}
$$

To simplify notation in the following derivation define

$$
\nabla s=\left(\begin{array}{l}
s_{1} \\
s_{2}
\end{array}\right)=\left(\begin{array}{l}
\partial_{x} m(u) \\
\partial_{y} m(u)
\end{array}\right)
$$

We seek to compute ${ }_{\nabla u} W_{\boldsymbol{s}}(\boldsymbol{s})$ in the E-L equation 4.16$)$, that is

$$
\begin{aligned}
\nabla_{u} W_{\boldsymbol{s}}(\boldsymbol{s}) & =\left(\begin{array}{c}
\nabla^{t} u W_{s_{1}} \\
\nabla^{t} u W_{s_{2}}
\end{array}\right)=\left(\begin{array}{c}
\nabla^{t} u\left(E_{s_{1}} D+E D_{s_{1}}\right) \\
\nabla^{t} u\left(E_{s_{2}} D+E D_{s_{2}}\right)
\end{array}\right) \\
& =\left(\begin{array}{c}
\nabla^{t} u E_{s_{1}} D \\
\nabla^{t} u E_{s_{2}} D
\end{array}\right)+E\left(\begin{array}{c}
\nabla^{t} u D_{s_{1}} \\
\nabla^{t} u D_{s_{2}}
\end{array}\right) .
\end{aligned}
$$


- The derivatives of $E$ are simple and read

$$
\begin{aligned}
& E_{s_{1}}=\partial_{s_{1}} \frac{1}{|\nabla s|}=-\frac{1}{|\nabla s|^{3}} s_{1}, \\
& E_{s_{2}}=\partial_{s_{2}} \frac{1}{|\nabla s|}=-\frac{1}{|\nabla s|^{3}} s_{2} .
\end{aligned}
$$

such that A.38 becomes

$$
{ }_{\nabla u} W_{\boldsymbol{s}}(\boldsymbol{s})=-\frac{1}{|\nabla s|^{3}}\left(\begin{array}{c}
s_{1} \nabla^{t} u D \\
s_{2} \nabla^{t} u D
\end{array}\right)+\frac{1}{|\nabla s|}\left(\begin{array}{c}
\nabla^{t} u D_{s_{1}} \\
\nabla^{t} u D_{s_{2}}
\end{array}\right) .
$$

- The derivatives $D_{s_{1}}$ and $D_{s_{2}}$ are considerably more cumbersome to compute, though straightforward. Start by expanding $D$ in terms of its eigendecomposition

$$
\begin{aligned}
D(\nabla s)=U \Lambda U^{t} & =\left(\begin{array}{cc}
v_{1} & w_{1} \\
v_{2} & w_{2}
\end{array}\right)\left(\begin{array}{cc}
\lambda_{1} & 0 \\
0 & \lambda_{2}
\end{array}\right)\left(\begin{array}{cc}
v_{1} & v_{2} \\
w_{1} & w_{2}
\end{array}\right) \\
& =\left(\begin{array}{cc}
v_{1}^{2} \lambda_{1}+w_{1}^{2} \lambda_{2} & v_{1} v_{2} \lambda_{1}+w_{1} w_{2} \lambda_{2} \\
v_{1} v_{2} \lambda_{1}+w_{1} w_{2} \lambda_{2} & v_{2}^{2} \lambda_{1}+w_{2}^{2} \lambda_{2}
\end{array}\right) \\
& =\left(\begin{array}{cc}
v_{1}^{2} & v_{1} v_{2} \\
v_{1} v_{2} & v_{2}^{2}
\end{array}\right) \lambda_{1}+\left(\begin{array}{cc}
w_{1}^{2} & w_{1} w_{2} \\
w_{1} w_{2} & w_{2}^{2}
\end{array}\right) \lambda_{2},
\end{aligned}
$$

where $v=\left(v_{1}, v_{2}\right)^{t}$ and $w=\left(w_{1}, w_{2}\right)^{t}$ are the corresponding orthonormal eigenvectors of GET. The eigenvalues of $D$ are given by $\lambda_{1,2}=\exp \left(-\left|\mu_{1,2}\right| / k^{2}\right)$ and

$$
\mu_{1}=\frac{1}{2}(\operatorname{tr}(G E T)+\alpha), \quad \mu_{2}=\frac{1}{2}(\operatorname{tr}(G E T)-\alpha),
$$

are the eigenvalues of the $G E T$-tensor with $\alpha=\sqrt{\operatorname{tr}(G E T)^{2}-4 \operatorname{det}(G E T)}$. The corresponding eigenvectors are then obtained by solving $G E T \tilde{v}=\mu_{1} \tilde{v}$. The eigendecomposition can be expressed as:

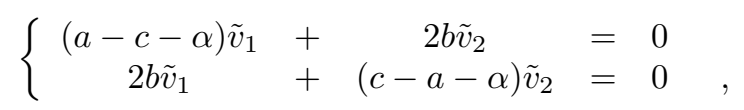

which result in

$$
v=\left(\begin{array}{l}
v_{1} \\
v_{2}
\end{array}\right)=\frac{1}{|\tilde{v}|} \tilde{v}, \quad \text { where } \quad \tilde{v}=\left(\begin{array}{c}
2 b \\
c-a+\alpha
\end{array}\right), \quad b \neq 0
$$

and

$$
w=\left(\begin{array}{l}
w_{1} \\
w_{2}
\end{array}\right)=\frac{1}{|\tilde{w}|} \tilde{w}, \quad \text { where } \quad \tilde{w}=\left(\begin{array}{c}
2 b \\
c-a-\alpha
\end{array}\right), \quad b \neq 0,
$$

for $b=0$ we have $\mu_{1}=a$ and $\mu_{2}=c$ and

$$
v=\left(\begin{array}{l}
v_{1} \\
v_{2}
\end{array}\right)=\left(\begin{array}{l}
1 \\
0
\end{array}\right), \quad b=0
$$


and

$$
w=\left(\begin{array}{l}
w_{1} \\
w_{2}
\end{array}\right)=\left(\begin{array}{l}
0 \\
1
\end{array}\right), \quad b=0 .
$$

- The component $\nabla^{t} u D_{s_{1}}$ then reads

$$
\begin{aligned}
\nabla^{t} u D_{s_{1}}=\nabla^{t} u & \left(\left(\partial_{s_{1}} v v^{t}\right) \lambda_{1}+v v^{t}\left(\partial_{s_{1}} \lambda_{1}\right)\right. \\
+ & \left.\left(\partial_{s_{1}} w w^{t}\right) \lambda_{2}+w w^{t}\left(\partial_{s_{1}} \lambda_{2}\right)\right) .
\end{aligned}
$$

Now, consider $D_{s_{1}}$ :

$$
\begin{aligned}
D_{s_{1}}= & \left(\begin{array}{cc}
2 v_{1}\left(\partial_{s_{1}} v_{1}\right) & \left(\partial_{s_{1}} v_{1}\right) v_{2}+v_{1}\left(\partial_{s_{1}} v_{2}\right) \\
\left(\partial_{s_{1}} v_{1}\right) v_{2}+v_{1}\left(\partial_{s_{1}} v_{2}\right) & 2 v_{2}\left(\partial_{s_{1}} v_{2}\right)
\end{array}\right) \lambda_{1} \\
+v v^{t}\left(\partial_{s_{1}} \lambda_{1}\right) & \\
+ & \left(\begin{array}{cc}
2 w_{1}\left(\partial_{s_{1}} w_{1}\right) & \left(\partial_{s_{1}} w_{1}\right) w_{2}+w_{1}\left(\partial_{s_{1}} w_{2}\right)
\end{array}\right) \lambda_{2} \\
\left(\partial_{s_{1}} w_{1}\right) w_{2}+w_{1}\left(\partial_{s_{1}} w_{2}\right) & 2 w_{2}\left(\partial_{s_{1}} w_{2}\right) \\
+w w^{t}\left(\partial_{s_{1}} \lambda_{2}\right), &
\end{aligned}
$$

where

$$
\begin{aligned}
\partial_{s_{1}} v_{1} & =\frac{\left(\partial_{s_{1}} \tilde{v}_{1}\right)|\tilde{v}|-\tilde{v}_{1}\left(\partial_{s_{1}}|\tilde{v}|\right)}{|\tilde{v}|^{2}}, \\
\partial_{s_{1}} w_{1} & =\frac{\left(\partial_{s_{1}} \tilde{w}_{1}\right)|\tilde{w}|-\tilde{w}_{1}\left(\partial_{s_{1}}|\tilde{w}|\right)}{|\tilde{w}|^{2}}, \\
\partial_{s_{1}} v_{2} & =\frac{\left(\partial_{s_{1}} \tilde{v}_{2}\right)|\tilde{v}|-\tilde{v}_{2}\left(\partial_{s_{1}}|\tilde{v}|\right)}{|\tilde{v}|^{2}}, \\
\partial_{s_{1}} w_{2} & =\frac{\left(\partial_{s_{1}} \tilde{w}_{2}\right)|\tilde{w}|-\tilde{w}_{2}\left(\partial_{s_{1}}|\tilde{w}|\right)}{|\tilde{w}|^{2}} .
\end{aligned}
$$

The eigenvector derivatives are obtained by differentiating the components $a, b$ and $c$ in A.34)-A.36). By expanding the derivatives of the eigenvectors we obtain

$$
\begin{aligned}
\partial_{s_{1}} \tilde{v}_{1} & =\partial_{s_{1}} 2 b \\
& =-\left(\partial_{y x} s_{1}+\partial_{y y} s_{2}\right) \\
\partial_{s_{1}} \tilde{v}_{2} & =\partial_{s_{1}}(c-a+\alpha) \\
& =\partial_{x x} s_{1}+\partial_{x y} s_{2}+\partial_{s_{1}} \alpha \\
\partial_{s_{1}} \tilde{w}_{1} & =\tilde{v}_{1} \\
\partial_{s_{1}} \tilde{w}_{2} & =\partial_{s_{1}}(c-a-\alpha) \\
& =\partial_{x x} s_{1}+\partial_{x y} s_{2}-\partial_{s_{1}} \alpha \\
\partial_{s_{1}}|\tilde{v}| & =|\tilde{v}|^{-1}\left(\tilde{v}_{1}\left(\partial_{s_{1}} \tilde{v}_{1}\right)+\tilde{v}_{2}\left(\partial_{s_{1}} \tilde{v}_{2}\right)\right) \\
\partial_{s_{1}}|\tilde{w}| & =|\tilde{w}|^{-1}\left(\tilde{w}_{1}\left(\partial_{s_{1}} \tilde{w}_{1}\right)+\tilde{w}_{2}\left(\partial_{s_{1}} \tilde{w}_{2}\right)\right) .
\end{aligned}
$$


The corresponding eigenvalue derivatives reads

$$
\begin{aligned}
\partial_{s_{1}} \lambda_{1,2} & =\partial_{s_{1}} \exp \left(-\left|\mu_{1,2}\right| / k^{2}\right) \\
& =-\frac{1}{k^{2}}\left(\partial_{s_{1}}\left|\mu_{1,2}\right|\right) \lambda_{1,2} \\
& =-\frac{\operatorname{sgn}\left(\mu_{1,2}\right)}{2 k^{2}}\left(\partial_{s_{1}} \operatorname{tr}(G E T)+\partial_{s_{1}} \alpha\right) \lambda_{1,2},
\end{aligned}
$$

where sgn is the signum function and

$$
\begin{aligned}
\partial_{s_{1}} \operatorname{tr}(G E T) & =\partial_{s_{1}}(a+c) \\
& =-\left(\partial_{x x} s_{1}+\partial_{x y} s_{2}\right), \\
\partial_{s_{1}} \alpha & =\partial_{s_{1}} \sqrt{\operatorname{tr}(G E T)^{2}-4 \operatorname{det}(G E T)} \\
& =\alpha^{-1}\left(\operatorname{tr}(G E T) \partial_{s_{1}} \operatorname{tr}(G E T)-2 \partial_{s_{1}} \operatorname{det}(G E T)\right), \\
\partial_{s_{1}} \operatorname{det}(G E T) & =\partial_{s_{1}}\left(a c-b^{2}\right) \\
& =-\left(\partial_{x x} s_{1}+\partial_{x y} s_{2}\right) c+b\left(\partial_{y x} s_{1}+\partial_{y y} s_{2}\right) .
\end{aligned}
$$

If $b=0$ the difference to the above derivation is that $\operatorname{det}(G E T)=a c$, thus $\partial_{s_{1}} \alpha$ and $\partial_{s_{1}} \operatorname{det}(G E T)$ should be modified accordingly.

- The component $\nabla^{t} s D_{s_{2}}$ then reads

$$
\begin{array}{rl}
\nabla^{t} s D_{s_{2}}=\nabla^{t} & s\left(\left(\partial_{s_{2}} v v^{t}\right) \lambda_{1}+v v^{t}\left(\partial_{s_{2}} \lambda_{1}\right)\right. \\
& \left.+\left(\partial_{s_{2}} w w^{t}\right) \lambda_{2}+w w^{t}\left(\partial_{s_{2}} \lambda_{2}\right)\right) .
\end{array}
$$

Now, consider $D_{s_{2}}$ :

$$
\begin{aligned}
D_{s_{2}}= & \left(\begin{array}{cc}
2 v_{1}\left(\partial_{s_{2}} v_{1}\right) & \left(\partial_{s_{2}} v_{1}\right) v_{2}+v_{1}\left(\partial_{s_{2}} v_{2}\right) \\
\left(\partial_{s_{2}} v_{1}\right) v_{2}+v_{1}\left(\partial_{s_{2}} v_{2}\right) & 2 v_{2}\left(\partial_{s_{2}} v_{2}\right)
\end{array}\right) \lambda_{1} \\
+v v^{t}\left(\partial_{s_{2}} \lambda_{1}\right) & \\
+ & \left(\begin{array}{cc}
2 w_{1}\left(\partial_{s_{2}} w_{1}\right) & \left(\partial_{s_{2}} w_{1}\right) w_{2}+w_{1}\left(\partial_{s_{2}} w_{2}\right) \\
\left(\partial_{s_{2}} w_{1}\right) w_{2}+w_{1}\left(\partial_{s_{2}} w_{2}\right) & 2 w_{2}\left(\partial_{s_{2}} w_{2}\right) \\
+w w^{t}\left(\partial_{s_{2}} \lambda_{2}\right) &
\end{array}\right. \\
&
\end{aligned}
$$

where

$$
\begin{aligned}
\partial_{s_{2}} v_{1} & =\frac{\left(\partial_{s_{2}} \tilde{v}_{1}\right)|\tilde{v}|-\tilde{v}_{1}\left(\partial_{s_{2}}|\tilde{v}|\right)}{|\tilde{v}|^{2}} \\
\partial_{s_{2}} w_{1} & =\frac{\left(\partial_{s_{2}} \tilde{w}_{1}\right)|\tilde{w}|-\tilde{w}_{1}\left(\partial_{s_{2}}|\tilde{w}|\right)}{|\tilde{w}|^{2}} \\
\partial_{s_{2}} v_{2} & =\frac{\left(\partial_{s_{2}} \tilde{v}_{2}\right)|\tilde{v}|-\tilde{v}_{2}\left(\partial_{s_{2}}|\tilde{v}|\right)}{|\tilde{v}|^{2}} \\
\partial_{s_{2}} w_{2} & =\frac{\left(\partial_{s_{2}} \tilde{w}_{2}\right)|\tilde{w}|-\tilde{w}_{2}\left(\partial_{s_{2}}|\tilde{w}|\right)}{|\tilde{w}|^{2}} .
\end{aligned}
$$


The eigenvector derivatives are obtained by differentiating the components $a, b$ and $c$ in A.34 - A.36. By expanding the derivatives of the eigenvectors we obtain

$$
\begin{aligned}
\partial_{s_{2}} \tilde{v}_{1} & =\partial_{s_{2}} 2 b \\
& =-\left(\partial_{x x} s_{1}+\partial_{x y} s_{2}\right) \\
\partial_{s_{2}} \tilde{v}_{2} & =\partial_{s_{2}}(c-a+\alpha) \\
& =-\left(\partial_{y x} s_{1}+\partial_{y y} s_{2}\right)+\partial_{s_{2}} \alpha \\
\partial_{s_{2}} \tilde{w}_{1} & =\partial_{s_{2}} \tilde{v}_{1} \\
\partial_{s_{2}} \tilde{w}_{2} & =\partial_{s_{2}}(c-a-\alpha) \\
& =-\left(\partial_{y x} s_{1}+\partial_{y y} s_{2}\right)-\partial_{s_{2}} \alpha \\
\partial_{s_{2}}|\tilde{v}| & =|\tilde{v}|^{-1}\left(\tilde{v}_{1}\left(\partial_{s_{2}} \tilde{v}_{1}\right)+\tilde{v}_{2}\left(\partial_{s_{2}} \tilde{v}_{2}\right)\right) \\
\partial_{s_{2}}|\tilde{w}| & =|\tilde{w}|^{-1}\left(\tilde{w}_{1}\left(\partial_{s_{2}} \tilde{w}_{1}\right)+\tilde{w}_{2}\left(\partial_{s_{2}} \tilde{w}_{2}\right)\right) .
\end{aligned}
$$

The corresponding eigenvalue derivatives reads

$$
\begin{aligned}
\partial_{s_{2}} \lambda_{1,2} & =\partial_{s_{2}} \exp \left(-\left|\mu_{1,2}\right| / k^{2}\right) \\
& =-\frac{1}{k^{2}}\left(\partial_{s_{2}}\left|\mu_{1,2}\right|\right) \lambda_{1,2} \\
& =-\frac{\operatorname{sgn}\left(\mu_{1,2}\right)}{2 k^{2}}\left(\partial_{s_{2}} \operatorname{tr}(G E T)+\partial_{s_{2}} \alpha\right) \lambda_{1,2}
\end{aligned}
$$

where

$$
\begin{aligned}
\partial_{s_{2}} \operatorname{tr}(G E T) & =\partial_{s_{2}}(a+c) \\
& =-\left(\partial_{y x} s_{1}+\partial_{y y} s_{2}\right), \\
\partial_{s_{2}} \alpha & =\partial_{s_{2}} \sqrt{\operatorname{tr}(G E T)^{2}-4 \operatorname{det}(G E T)} \\
& =\alpha^{-1}\left(\operatorname{tr}(G E T) \partial_{s_{2}} \operatorname{tr}(G E T)-2 \partial_{s_{2}} \operatorname{det}(G E T)\right), \\
\partial_{s_{2}} \operatorname{det}(G E T) & =\partial_{s_{2}}\left(a c-b^{2}\right) \\
& =-a\left(\partial_{y x} s_{1}+\partial_{y y} s_{2}\right)+b\left(\partial_{x x} s_{1}+\partial_{x y} s_{2}\right) .
\end{aligned}
$$

If $b=0$ the difference to the above derivation is that $\operatorname{det}(G E T)=a c$, thus $\partial_{s_{2}} \alpha$ and $\partial_{s_{2}} \operatorname{det}(G E T)$ should be modified accordingly. 


\section{Chapter 16}

\section{Bibliography}

[1] F. Åström. A Variational Approach to Image Diffusion in Non-Linear Domains. Linköping studies in science and technology. thesis no. 1594, Linköping University, Sweden, 2013.

[2] F. Åström, G. Baravdish, and M. Felsberg. On Tensor-Based PDEs and their Corresponding Variational Formulations with Application to Color Image Denoising. In European Conference on Computer Vision (ECCV), volume 7574, pages 215-228, Firenze, 2012. LNCS, Springer Berlin/Heidelberg.

[3] F. Åström, G. Baravdish, and M. Felsberg. A tensor variational formulation of gradient energy total variation. In X.-C. Tai, E. Bae, T. Chan, and M. Lysaker, editors, Energy Minimization Methods in Computer Vision and Pattern Recognition (EMMCVPR), volume 8932 of Lecture Notes in Computer Science, pages 307-320. Springer International Publishing, 2015.

[4] F. Åström and M. Felsberg. On the Choice of Tensor Estimation for Corner Detection, Optical Flow and Denoising. In Workshop on Emerging Topics in Image Restoration and Enhancement (IREw 2014) in conjunction with Asian Conference on Computer Vision (ACCV) (Accepted), 2014.

[5] F. Åström, M. Felsberg, and G. Baravdish. Domain-Dependent Anisotropic Diffusion. Journal of Mathematical Imaging and Vision (submitted), 2014.

[6] F. Åström, M. Felsberg, G. Baravdish, and C. Lundström. Visualization Enhancing Diffusion. In Swedish Symposium on Image Analysis (SSBA), Stockholm, 2012.

[7] F. Åström, M. Felsberg, G. Baravdish, and C. Lundström. Targeted iterative filtering. In A. Kuijper, K. Bredies, T. Pock, and H. Bischof, editors, Scale Space and Variational Methods in Computer Vision (SSVM), volume 7893 of Lecture Notes in Computer Science, pages 1-11. Springer Berlin Heidelberg, 2013. 
[8] F. Åström, M. Felsberg, and R. Lenz. Color Persistent Anisotropic Diffusion of Images. In A. Heyden and F. Kahl, editors, Image Analysis, volume 6688 of Lecture Notes in Computer Science, pages 262-272. Springer, 2011.

[9] F. Åström, M. Felsberg, and R. Lenz. Color Persistent Anisotropic Diffusion of Images. In Swedish Symposium on Image Analysis (SSBA), Linköping, 2011 .

[10] F. Åström and R. Köker. A parallel neural network approach to prediction of Parkinsons Disease. Expert systems with applications, 38(10):12470-12474, 2011.

[11] F. Åström, V. Zografos, and M. Felsberg. Density Driven Diffusion. In 18th Scandinavian Conferences on Image Analysis, 2013, volume 7944 of Lecture Notes in Computer Science, pages 718-730, 2013.

[12] F. Åström, V. Zografos, and M. Felsberg. Image Denoising via Density Driven Diffusion. In Swedish Symposium on Image Analysis (SSBA), Göteborg, 2013.

[13] S. Baker, D. Scharstein, J. P. Lewis, S. Roth, M. J. Black, and R. Szeliski. A Database and Evaluation Methodology for Optical Flow. International Journal of Computer Vision, 92(1):1-31, 2011.

[14] C. Ballester, M. Bertalmio, V. Caselles, G. Sapiro, and J. Verdera. Filling-in by joint interpolation of vector fields and gray levels. IEEE Transactions on Image Processing, 10(8):1200-1211, 2001.

[15] G. Baravdish, O. Svensson, and F. Åström. On Backward $p(x)$-Parabolic Equations for Image Enhancement. Numerical Functional Analysis and Optimization, 36(2):147-168, 2015.

[16] J. Bigün and G. H. Granlund. Optimal Orientation Detection of Linear Symmetry. In International Conference on Computer Vision (ICCV), pages 433-438, London, Great Britain, 1987.

[17] M. J. Black and A. Rangarajan. On the Unification of Line Processes, Outlier Rejection, and Robust Statistics with Applications in Early Vision. International Journal of Computer Vision, 19(1):57-91, 1996.

[18] M. J. Black, G. Sapiro, D. H. Marimont, and D. Heeger. Robust anisotropic diffusion. IEEE Transactions on Image Processing, 7(3):421-432, Mar. 1998.

[19] A. C. Bovik and P. Maragos. Conditions for positivity of an energy operator. IEEE Transactions on Signal Processing, 42(2):469-471, Feb. 1994.

[20] K. Bredies, K. Kunisch, and T. Pock. Total Generalized Variation. SIAM Journal of Imaging Sciences, 3(3):492-526, Sept. 2009.

[21] A. Buades, B. Coll, and J. Morel. A Review of Image Denoising Algorithms, with a New One. Multiscale Modeling \& Simulation, 4(2):490-530, 2005. 
[22] A. Buades, B. Coll, and J.-M. Morel. A non-local algorithm for image denoising. In Computer Vision and Pattern Recognition (CVPR), volume 2, pages $60-65$ vol. 2 , 2005 .

[23] A. Chambolle and T. Pock. A First-Order Primal-Dual Algorithm for Convex Problems with Applications to Imaging. Journal of Mathematical Imaging and Vision, 40(1):120-145, 2011.

[24] T. Chan, S. Esedoglu, F. Park, and A. Yip. Total Variation Image Restoration: Overview and Recent Developments. In N. Paragios, Y. Chen, and O. Faugeras, editors, Handbook of Mathematical Models in Computer Vision, chapter 2, pages 17-31. Springer US, 2006.

[25] T. F. Chan and C.-K. Wong. Total variation blind deconvolution. IEEE Transactions on Image Processing, 7(3):370-375, 1998.

[26] D. Comaniciu, P. Meer, and S. Member. Mean shift: A robust approach toward feature space analysis. IEEE transactions on Pattern Analysis and Machine Intelligence (PAMI), 24:603-619, 2002.

[27] K. Dabov, A. Foi, V. Katkovnik, and K. Egiazarian. Image denoising with block-matching and 3D filtering. In SPIE Electronic Imaging 6064, 2006.

[28] K. Dabov, A. Foi, V. Katkovnik, and K. Egiazarian. Color Image Denoising via Sparse 3D Collaborative Filtering with Grouping Constraint in Luminance-Chrominance Space. In International Conference on Image Processing (ICIP), 2007.

[29] P. E. Debevec and J. Malik. Recovering high dynamic range radiance maps from photographs. In SIGGRAPH '97, pages 369-378, 1997.

[30] M. V. den Bergh, X. Boix, G. Roig, B. de Capitani, and L. V. Gool. SEEDS: Superpixels Extracted via Energy-Driven Sampling. In A. W. Fitzgibbon, S. Lazebnik, P. Perona, Y. Sato, and C. Schmid, editors, European Conference on Computer Vision (ECCV), volume 7578 of LNCS, pages 13-26. Springer, 2012.

[31] J. M. DiCarlo and B. A. Wandell. Rendering high dynamic range images. In SPIE Image Sensors 3965, pages 392-401, 2000.

[32] M. Felsberg. Spatio-featural scale-space. In Scale Space and Variational Methods in Computer Vision (SSVM), pages 808-819. Springer Berlin/Heidelberg, 2009.

[33] M. Felsberg. Autocorrelation-Driven Diffusion Filtering. IEEE Transactions on Image Processing, 20(7):1797-1806, 2011.

[34] M. Felsberg, P.-E. Forssén, and H. Scharr. Channel Smoothing: Efficient Robust Smoothing of Low-Level Signal Features. IEEE Transactions on Pattern Analysis and Machine Intelligence (PAMI), 28(2):209-222, 2006. 
[35] M. Felsberg and G. Granlund. POI detection using channel clustering and the 2D energy tensor. In Pattern recognition: 26th DAGM symposium, pages 103-110. LNCS, Springer Berlin/Heidelberg, 2004.

[36] M. Felsberg and E. Jonsson. Energy Tensors: Quadratic, Phase Invariant Image Operators. In Pattern Recognition, volume 3663 of LNCS, pages 493500. Springer, 2005.

[37] M. Felsberg and U. Köthe. GET: The connection between monogenic scalespace and Gaussian derivatives. In R. Kimmel, N. Sochen, and J. Weickert, editors, Scale Space and PDE Methods in Computer Vision, volume 3459 of LNCS, pages 192-203. LNCS, 2005.

[38] W. Förstner. Image Preprocessing for Feature Extraction in Digital Intensity, Color and Range Images. In D. Athanasios, G. Armin, and S. Fernando, editors, Geomatic Method for the Analysis of Data in the Earth Sciences, volume 95 of LNES, pages 165-189. Springer, 2000.

[39] W. Förstner and E. Gülch. A fast operator for detection and precise location of distinct points, corners and centres of circular features. In ISPRS Intercommission, Workshop, Interlaken, pp. 149-155., 1987.

[40] J. Gårding and T. Lindeberg. Direct computation of shape cues using scaleadapted spatial derivative operators. International Journal of Computer Vision (IJCV), 17(2):163-191, Feb. 1996.

[41] R. C. Gonzalez and R. E. Woods. Digital Image Processing - $3 d$ edition. Pearson International Edition, 2008.

[42] G. H. Granlund. An Associative Perception-Action Structure using a Localized Space Variant Information Representation. In International Workshop on Algebraic Frames for the Perception Action Cycle (AFPAC), Sept. 2000.

[43] G. H. Granlund and H. Knutsson. Signal processing for computer vision. Kluwer, 1995.

[44] M. Grasmair and F. Lenzen. Anisotropic Total Variation Filtering. Applied Mathematics \& Optimization, 62(3):323-339, 2010.

[45] J. Hadamard. Sur les problèmes aux dérivés partielles et leur signification physique. Princeton University Bulletin, 13:49-52, 1902.

[46] C. Harris and M. Stephens. A combined corner and edge detector. In Fourth Alvey Vision Conference, pages 147-151, 1988.

[47] C. Heinemann, F. Åström, G. Baravdish, K. Krajsek, M. Felsberg, and H. Scharr. Using Channel Representations in Regularization Terms - A Case Study on Image Diffusion. Proceedings of the 9th International Conference on Computer Vision Theory and Applications (VISAPP), pages 48-55, 2014. 
[48] B. K. P. Horn and B. G. Schunk. Determining Optical Flow. Artificial Intelligence, 17:185-203, 1981.

[49] R. A. Horn and C. R. Johnson, editors. Matrix Analysis. Cambridge University Press, New York, NY, USA, 1986.

[50] T. Iijima. Basic theory of pattern observation. In Papers of Technical Group on Automata and Automatic Control, IECE, Japan, 1959.

[51] J. F. Kaiser. On a simple algorithm to calculate the 'energy' of a signal. In International Conference on Acoustics, Speech, and Signal Processing (ICASSP), pages 381-384 vol.1, Apr. 1990.

[52] M. Kass, A. Witkin, and D. Terzopoulos. Snakes: Active contour models. International Journal of Computer Vision, 1(4):321-331, 1988.

[53] J. Kačur and K. Mikula. Slowed Anisotropic Diffusion. In Scale-Space Theory in Computer Vision, volume 1252 of LNCS, pages 357-360. Springer Berlin / Heidelberg, 1997.

[54] R. Kimmel, R. Malladi, and N. Sochen. Images as Embedded Maps and Minimal Surfaces: Movies, Color, Texture, and Volumetric Medical Images. International Journal of Computer Vision, 39(2):111-129, 2000.

[55] H. Knutsson. Representing Local Structure Using Tensors. In The 6th Scandinavian Conference on Image Analysis (SCIA), pages 244-251, Oulu, Finland, 1989.

[56] H. Knutsson, C.-F. Westin, and M. Andersson. Representing Local Structure Using Tensors II. In A. Heyden and F. Kahl, editors, Image Analysis, volume 6688 of Lecture Notes in Computer Science, pages 545-556. Springer Berlin Heidelberg, 2011.

[57] J. J. Koenderink. The Structure of Images. Biological Cybernetics, 370(50):363-370, 1984.

[58] X. Kong, K. Li, Q. Yang, W. Liu, and M.-H. Yang. A New Image Quality Metric for Image Auto-Denoising. In International Conference on Computer Vision (ICCV), pages 2888-2895, 2013.

[59] K. Krajsek and H. Scharr. Diffusion Filtering Without Parameter Tuning : Models and Inference Tools. In Computer Vision and Pattern Recognition (CVPR), pages 2536-2543, San Francisco, 2010.

[60] G. Krieger and C. Zetzsche. Nonlinear image operators for the evaluation of local intrinsic dimensionality. Image Processing, 5(6):1026-42, Jan. 1996.

[61] S. Lefkimmiatis, A. Roussos, M. Unser, and P. Maragos. Convex Generalizations of Total Variation Based on the Structure Tensor with Applications to Inverse Problems. In A. Kuijper, K. Bredies, T. Pock, and H. Bischof, editors, Scale Space and Variational Methods in Computer Vision (SSVM), pages 48-60, 2013. 
[62] R. Lenz and P. L. Carmona. Hierarchical S(3)-Coding of RGB Histograms. In Selected papers from International Conference on Computer Vision Theory and Applications (VISAPP), volume 68, pages 188-200. Springer, 2010.

[63] T. Lindeberg. Scale-Space Theory in Computer Vision. Kluwer international series in engineering and computer science: Robotics: Vision, manipulation and sensors. Springer, 1993.

[64] B. D. Lucas and T. Kanade. An Iterative Image Registration Technique with an Application to Stereo Vision. In Proceedings of the rth International Joint Conference on Artificial Intelligence (IJCAI) - Volume 2, pages 674-679, San Francisco, CA, USA, 1981. Morgan Kaufmann Publishers Inc.

[65] D. Martin, C. Fowlkes, D. Tal, and J. Malik. A Database of Human Segmented Natural Images and its Application to Evaluating Segmentation Algorithms and Measuring Ecological Statistics. In Proc. 8th Internatioanl Conference on Computer Vision (ICCV), volume 2, pages 416-423, 2001.

[66] R. Mester, C. Conrad, and A. Guevara. Multichannel segmentation using contour relaxation: fast super-pixels and temporal propagation. In SCIA, pages 250-261, 2011.

[67] K. Mikolajczyk. www.robots.ox.ac.uk/ vgg/research/affine, 2014.

[68] K. Mikolajczyk and C. Schmid. A performance evaluation of local descriptors. IEEE Transactions on Pattern Analysis and Machine Intelligence (PAMI), 27(10):1615-1630, 2005.

[69] C. Moler and C. Van Loan. Nineteen Dubious Ways to Compute the Exponential of a Matrix, Twenty-Five Years Later. SIAM Review, 45(1):3-49, 2003.

[70] K. Nordberg. Signal Representation and Processing using Operator Groups. $\mathrm{PhD}$ thesis, Linköping University, Sweden, SE-581 83 Linköping, Sweden, 1995 .

[71] A. Olmos and F. A. A. Kingdom. A biologically inspired algorithm for the recovery of shading and reflectance images. Perception, 33(12):1463-1473, 2004 .

[72] P. Perona, J. Malik, and P. Pietro. Scale-space and edge detection using anisotropic diffusion. IEEE transactions on Pattern Analysis and Machine Intelligence, 12(7):629-639, July 1990.

[73] N. Pettersson and F. Åström. A system for Real-Time Surveillance DeWeathering. In Swedish Symposium on Image Analysis (SSBA), Luleå, 2014.

[74] V. Podlozhnyuk. Image convolution with CUDA, NVIDIA Corporation white paper, v1.0, 2007. 
[75] M. Prokop and M. Galanski. Spiral and Multislice Computed Tomography of the Body. Thieme Verlag, 2003.

[76] A. I. Renner. Anisotropic Diffusion in Riemannian Colour Space. PhD thesis, Ruprecht-Kars-Universität, Heidelberg, 2003.

[77] S. Roth and M. J. Black. Fields of Experts: A Framework for Learning Image Priors. In Computer Vision and Pattern Recognition (CVPR), pages 860-867 vol. 2, 2005.

[78] A. Roussos and P. Maragos. Tensor-based image diffusions derived from generalizations of the total variation and beltrami functionals. In International Conference on Image Processing (ICIP), pages 4141-4144, Hong Kong, 2010.

[79] L. I. Rudin, S. Osher, E. Fatemi, and F. Emad. Nonlinear total variation based noise removal algorithms. Physica D, 60(1-4):259-268, Nov. 1992.

[80] H. Scharr, M. J. Black, and H. W. Haussecker. Image statistics and anisotropic diffusion. In Ninth IEEE International Conference on Computer Vision (ICCV), pages $840-847$ vol.2, 2003.

[81] O. Scherzer and J. Weickert. Relations Between Regularization and Diffusion Filtering. Journal of Mathematical Imaging and Vision, 12(1):43-63, 2000.

[82] G. Sharma. Color fundamentals for digital imaging. In G. Sharma and R. Bala, editors, Digital Color Imaging Handbook, Electrical Engineering \& Applied Signal Processing Series, chapter 1. Taylor \& Francis, 2010.

[83] J. Shi and C. Tomasi. Good features to track. In Computer Vision and Pattern Recognition (CVPR), pages 593-600, June 1994.

[84] N. Sochen, R. Kimmel, and R. Malladi. A general framework for low level vision. IEEE Transactions on Image Processing, 7(3):310-318, Mar. 1998.

[85] W. A. Strauss. Partial Differential Equations: An Introduction. Wiley, 2007.

[86] D. Sun, S. Roth, and M. J. Black. Secrets of optical flow estimation and their principles. In Computer Vision and Pattern Recognition (CVPR), pages 2432-2439, 2010.

[87] B. Tang, G. Sapiro, and V. Caselles. Color image enhancement via chromaticity diffusion. IEEE Transactions on Image Processing on Image Processing, 10(5):701-707, 2001.

[88] A. N. Tikhonov. Solution of incorrectly formulated problems and the regularization method. Soviet Math. Dokl., 4:1035-1038, 1963.

[89] C. Tomasi and T. Kanade. Detection and Tracking of Point Features. Technical report, Carnegie Mellon University Technical Report CMU-CS-91-132, 1991. 
[90] C. Tomasi and R. Manduchi. Bilateral filtering for gray and color images. In 6 'th International Conference on Computer Vision (ICCV), pages 839-846, Jan. 1998.

[91] D. Tschumperlé and R. Deriche. Vector-valued Image Regularization with PDE's: A Common Framework for Different Applications. In Conference on Computer Vision and Pattern Recognition (CVPR), pages 651-656, 2003.

[92] K. E. A. van de Sande, T. Gevers, and C. G. M. Snoek. Evaluating Color Descriptors for Object and Scene Recognition. IEEE Transactions on Pattern Analysis and Machine Intelligence (PAMI), 32(9):1582-1596, Sept. 2010.

[93] M. Vollmer and K. P. Möllmann. Infrared Thermal Imaging: Fundamentals, Research and Applications. John Wiley \& Sons, 2010.

[94] Z. Wang, A. C. Bovik, H. R. Sheikh, and E. P. Simoncelli. Image quality assessment: from error visibility to structural similarity. IEEE Transactions on Image Processing, 13(4):600-612, Apr. 2004.

[95] J. Weickert. Anisotropic Diffusion in Image Processing. Teubner-Verlag, Stuttgart, Germany, 1998.

[96] J. Weickert. Coherence-enhancing diffusion of colour images. Image and Vision Computing, 17(3-4):201-212, 1999.

[97] J. Weickert. Nonlinear Diffusion Filtering. In B. Jähne, H. Haussecker, and P. Beissler, editors, Signal processing and pattern recognition, Handbook of Computer Vision and Applications, chapter 15, pages 423-451. Academic Press, 1999.

[98] J. Weickert. A Scheme for Coherence-Enhancing Diffusion Filtering with Optimized Rotation Invariance. Journal of Visual Communication and Image Representation, 13(1-2):103-118, 2002.

[99] W. Xi, X. Mingyuan, W. Wu, and J. Zhou. Nonlocal Mean Image Denoising Using Anisotropic Structure Tensor. Advances in Optical Technologies, 2013(794728):6, 2013.

[100] S. C. Zhu and D. Mumford. Prior Learning and Gibbs Reaction-Diffusion. IEEE transactions on Pattern Analysis and Machine Intelligence (PAMI), 19:1236-1250, 1997. 